BNL C-AD/AP/49

Fermilab Report TM-2158

\title{
VLHC Accelerator Physics
}

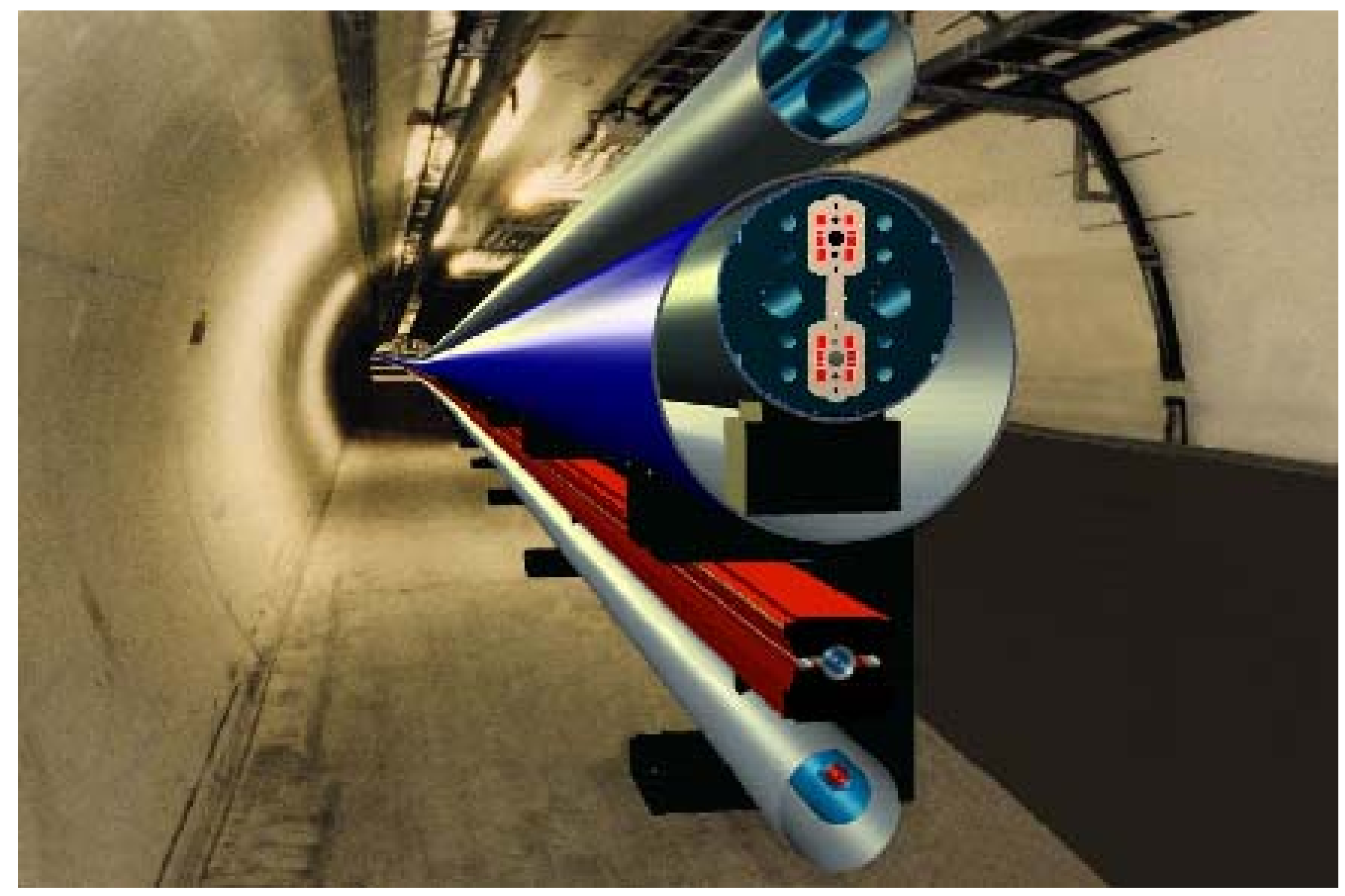

BNL: M. Blaskiewicz, A. Drees, W. Fischer, R. Gupta, S. Peggs, F. Pilat, V. Ptitsyn, S. Tepikian, D. Trbojevic, S.Y. Zhang FNAL: N. Gelfand, J. Johnstone, L. Michelotti, K.Y. Ng, T. Sen, V. Shiltsev, M. Syphers, M. Xiao UNM: M. Vogt

Editors: S. Peggs and M. Syphers

June 29, 2001 


\section{Contents}

1 MACHINE LAYOUTS $\quad 2$

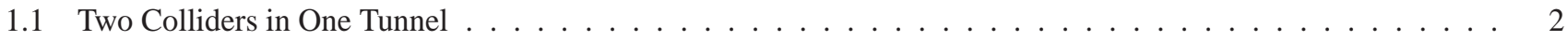

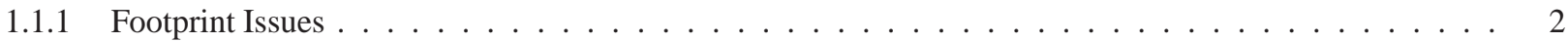

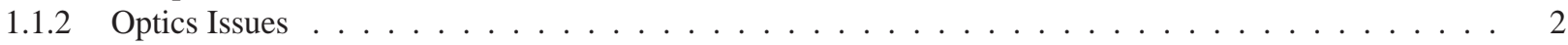

1.1 .3 Design Approach . . . . . . . . . . . . . . . . . . . . . . . . 3

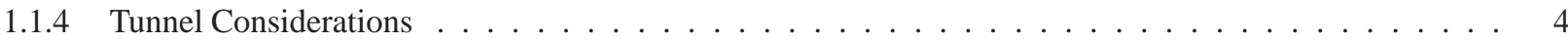

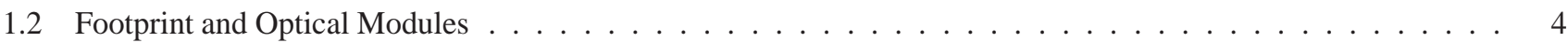

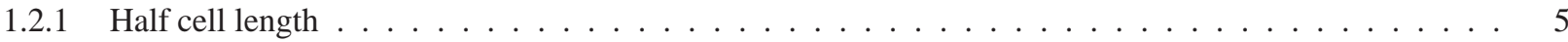

2 STAGE 1: THE LOW-FIELD RING $\quad 11$

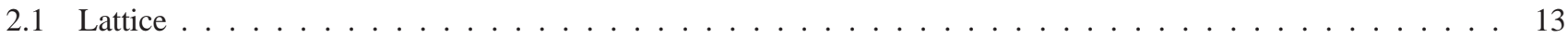

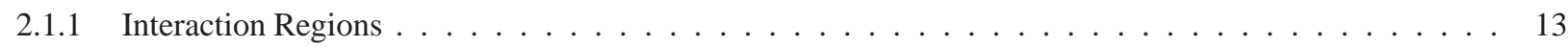

2.1 .2 Injection $/$ Abort $\ldots \ldots \ldots \ldots \ldots \ldots \ldots \ldots \ldots \ldots \ldots$

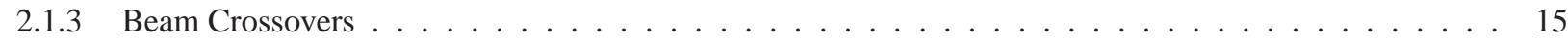

2.1.4 Miscellaneous Insertions . . . . . . . . . . . . . . . . . . . . . . . . . . . . . . 15

2.2 Dynamic Aperture and Field Quality . . . . . . . . . . . . . . . . . . . . . . . 18

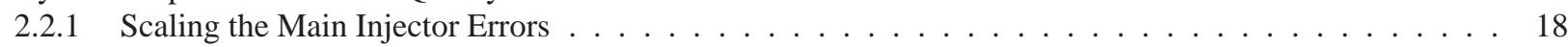

2.2 .2 Dynamic Aperture at Injection . . . . . . . . . . . . . . . . . . . . . . . . . 19

2.2 .3 Dynamic Aperture at Collision . . . . . . . . . . . . . . . . . . . . . . . 22

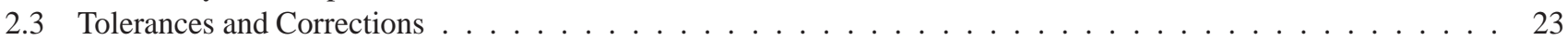

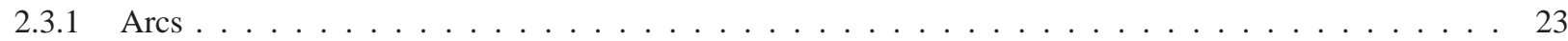

2.3 .2 Interaction Regions . . . . . . . . . . . . . . . . . . . . . . . . . . 27

2.4 Ground Motion and Emittance Growth . . . . . . . . . . . . . . . . . . . . . . . . . . . . . . . . . . . . . . . . 29

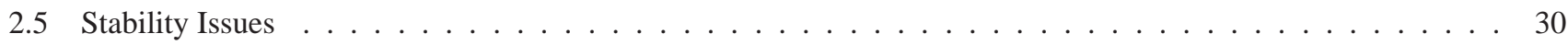

2.5 .1 The Rings, Injection, and $\mathrm{RF} \ldots \ldots \ldots \ldots \ldots \ldots \ldots \ldots$

2.5 .2 Resistive Wall . . . . . . . . . . . . . . . . . . . . . . . . . . . 30

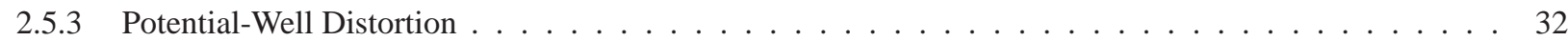

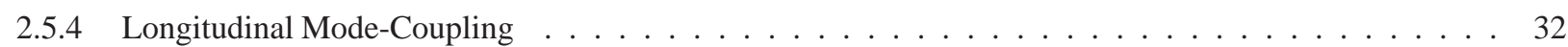

2.5 .5 Transverse Mode-Coupling . . . . . . . . . . . . . . . . . . . . . . . 35

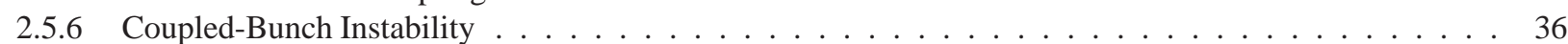

2.5 .7 Longitudinal Head-Tail . . . . . . . . . . . . . . . . . . . . . . . . . . . 36

2.5.8 Laslett Tune Shifts and Other Effects . . . . . . . . . . . . . . . . . . . . . . . . . . 39

2.5.9 Bunch Coalescence . . . . . . . . . . . . . . . . . . . . . . . . . . . . . . . . . . 39

2.6 Influence of Magnetic Fields on Damping Rates . . . . . . . . . . . . . . . . . . . . . . . 42

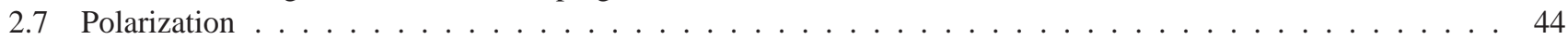

3 STAGE 2: THE HIGH-FIELD RING $\quad 45$

3.1 Luminosity Versus Energy . . . . . . . . . . . . . . . . . . . . . . . . . . . . 45

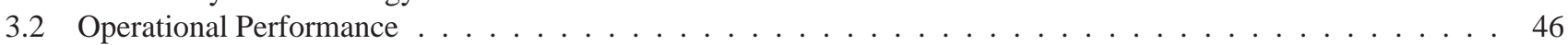

3.2.1 The Head On Beam-Beam Interaction . . . . . . . . . . . . . . . . . . . . . . . . . . 47

3.2.2 Crossing Angles and Parasitic Beam-Beam Collisions. . . . . . . . . . . . . . . . . . . 51

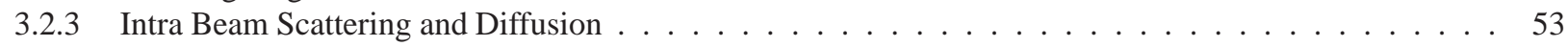

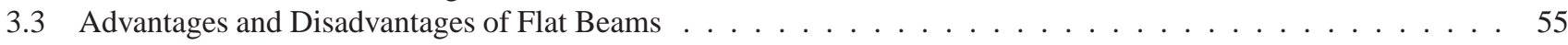

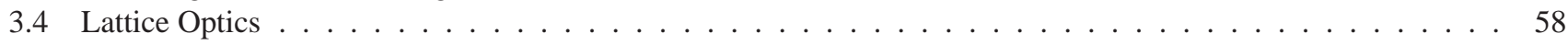

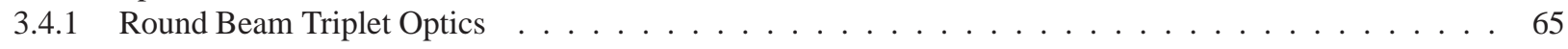

3.5 Interaction Region Magnets . . . . . . . . . . . . . . . . . . . . . . . . . . 67

3.6 Dynamic Aperture and Field Quality . . . . . . . . . . . . . . . . . . . . . . . . . . . . . . . 69

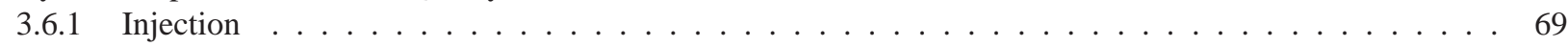

3.6 .2 Minimum Injection Energy . . . . . . . . . . . . . . . . . . . . . . 72

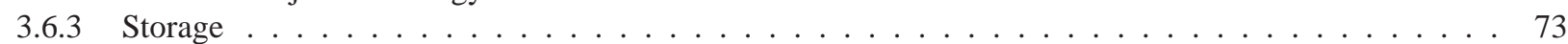

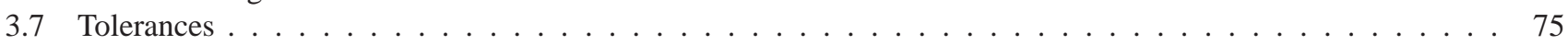

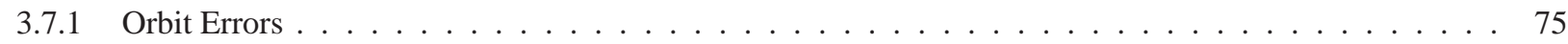


3.7.2 Tune Error, Coupling and IR Chromatic Effects . . . . . . . . . . . . . . . . . . . 76

3.7 .3 Corrector Strengths . . . . . . . . . . . . . . . . . . . . . . . . 76

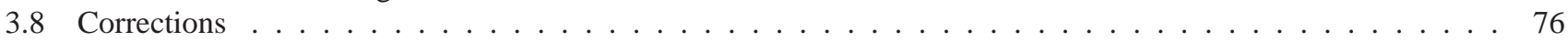

3.8.1 Arc Correction System . . . . . . . . . . . . . . . . . . . . . . . 77

3.8.2 Interaction Region Correction System . . . . . . . . . . . . . . . . . . . . . . . . . 78

3.9 Beam Stability . . . . . . . . . . . . . . . . . . . . . . . . . . . 79

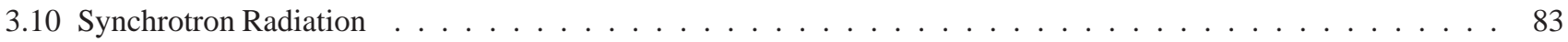

3.11 Electron Cloud Instability . . . . . . . . . . . . . . . . . . . . . . . . . . 86

3.12 Energy Deposition at the Interaction Region . . . . . . . . . . . . . . . . . . . . . . 89

3.13 Beam Transfer from Low Field to High Field . . . . . . . . . . . . . . . . . . . . . . . . . . . . . . 92

3.14 Fundamental Problems, and Future Research Fronts . . . . . . . . . . . . . . . . . . . . . . . . . . . . 97

4 Parameters 98

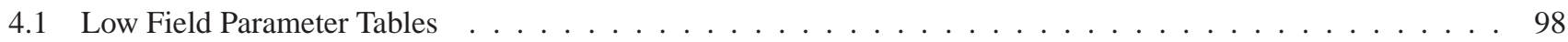

4.2 High Field Parameter Tables . . . . . . . . . . . . . . . . . . . . . . . . . . . . . 102

4.3 Magnet Reference Harmonics . . . . . . . . . . . . . . . . . . . . . . . . . 108 


\section{Preface}

A six-month design study for a future high energy hadron collider was initiated by the Fermilab director in October 2000. The request was to study a staged approach where a large circumference tunnel is built that initially would house a low field $(\sim 2 \mathrm{~T})$ collider with center-of-mass energy greater than $30 \mathrm{TeV}$ and a peak (initial) luminosity of $10^{34} \mathrm{~cm}^{-2} \mathrm{~s}^{-1}$. The tunnel was to be scoped, however, to support a future upgrade to a center-of-mass energy greater than $150 \mathrm{TeV}$ with a peak luminosity of $2 \times 10^{34} \mathrm{~cm}^{-2} \mathrm{sec}^{-1}$ using high field $(\sim 10 \mathrm{~T})$ superconducting magnet technology.

In a collaboration with Brookhaven National Laboratory and Lawrence Berkeley National Laboratory, a report of the Design Study was produced by Fermilab in June 2001. ${ }^{1}$ The Design Study focused on a Stage 1, $20 \times 20 \mathrm{TeV}$ collider using a 2-in-1 transmission line magnet and leads to a Stage $2,87.5 \times 87.5 \mathrm{TeV}$ collider using $10 \mathrm{~T} \mathrm{Nb}_{3} \mathrm{Sn}$ magnet technology.

The article that follows is a compilation of accelerator physics designs and computational results which contributed to the Design Study. Many of the parameters found in this report evolved during the study, and thus slight differences between this text and the Design Study report can be found. The present text, however, presents the major accelerator physics issues of the

Very Large Hadron Collider as examined by the Design Study collaboration and provides a basis for discussion and further studies of VLHC accelerator parameters and design philosophies.

Steve Peggs and Mike Syphers, editors.

${ }^{1}$ P.J. Limon, et al., "Design Study for a Staged Very Large Hadron Collider,” Fermilab-TM-2149, June 2001. 


\section{MACHINE LAYOUTS}

\subsection{Two Colliders in One Tunnel}

The desire of the Design Study [1] is to generate a low field (approximately $2 \mathrm{~T}$ ) collider design in a tunnel which also can be used for a future high field (approximately $10 \mathrm{~T}$ ) collider. While the second collider is far in the future, one needs to anticipate its requirements as best one can before laying out the components of the first machine since they ultimately must reside in same tunnel. This entails anticipating the geometric requirements, beam optics requirements, and physical space requirements for future technical equipment - much of which may not be known yet. In the paragraphs that follow, we discuss some of the issues that were involved with the designs and describe part of the design strategy followed for making some of the choices for this study. Further optical details can be found in this chapter, in the following chapter, and elsewhere [2].

\subsubsection{Footprint Issues}

For the Design Study it has been envisioned that the Low Field (LF) ring will be comprised of long, combined function magnets - the bending of the central trajectory and the focusing of the particle beam will be performed using gradient magnets with a central field strength on the order of $2 \mathrm{~T}$. The High Field (HF) ring will use separated function magnets - dipole magnets for bending the central trajectory and quadrupole magnets for focusing. Since over 75 percent of the circumference will be made up of bending magnets, the design of the standard bending modules in the arcs of both colliders must be performed simultaneously. Both rings must have the same average radius of curvature so that they follow each other. The same cell length - the distance over which the bending and focusing characteristics repeat themselves - is used for both rings. Although the LF and HF will use different length bending magnets the arcs of the two colliders will follow each other with minimal relative transverse offsets by maintaining the same average radius of curvature.

One complication of the geometry comes about at the transition regions between bending and non-bending portions of the accelerators. Special optical modules - Dispersion Suppressors (DS) - are used to bring the orbits of off-momentum particles to coincide with each other in the long straight sections. Since the LF and HF designs have different focusing characteristics, the dispersion suppressors must also be designed simultaneously to ensure that the LF and HF orbits line up appropriately when entering the long straight sections for the Interaction Regions (IR) and Utility Regions (UT).

While the size of the arcs is determined primarily by the low magnetic field of the first stage collider, the lengths of the major straight sections are determined primarily by the high magnetic rigidity of the beam in the second stage collider. The interaction regions must be made long enough to accommodate trajectory and optics manipulations of high energy proton beams using reasonable guesses for sizes of future high energy, high luminosity detectors. The utility straight section regions must be made long enough to provide space for extracting a very high energy proton beam toward a beam dump and for beam scraping and beam instrumentation at high energy. All of these functions will be required for the LF collider as well, of course, but the technologies used for injection, extraction, acceleration, and so forth will be similar for the two rings. Thus, the requirements of the HF design will set the scale for the straight sections.

During the design of the two colliders, "virtual survey monuments" are used to ensure that geometric differences between their footprints are small and tolerable. These "markers" are placed at strategic locations in the layout of each ring - interaction points, beginning of arcs, etc. - and at each design iteration the geometries of the rings are checked to ensure that these markers have not moved. This method guarantees that the two accelerators line up at the desired locations.

\subsubsection{Optics Issues}

In addition to the geometric layout of the two rings, many other optical properties must also be simultaneously decided. The low field ring uses gradient magnets in the arcs of the collider, while the high field ring uses quadrupole FODO cells. For simplicity of instrumentation, power distribution, cable runs, etc., it is desirable for the maximum beam size locations in the HF arc to correspond with those in the LF arc. Traditionally, dispersion suppressor regions are made using "missing magnet" scenarios. The fraction of bending magnets to leave out depends upon the phase advance per cell. In combined function lattices, such as in the LF design, the bending magnets also provide focusing, so this tactic must be modified. While combined function designs of dispersion suppressors have been performed in the past (e.g., Fermilab Recycler), the choice of cell phase advance and cell lengths must be settled upon for both rings to ensure a consistent dispersion suppressor design. (Obviously, this is an issue for both optics and geometry as well.) This is another reason that it was chosen to give the two colliders equal cell lengths and equal betatron phase advances per cell.

Each collider, LF and HF, is made of two separate, but linked, accelerators. The choice must be made as to whether adjacent magnets have the same or opposite focusing characteristic with respect each other for the beam traveling through them. This 
affects the matching of sections going into and out of straight sections, as well as the design of the beam transfer system joining the LF and HF rings. The decision must be made in coordination with the magnet design effort.

Finally, the magnet choice for the LF collider is a horizontally separated two-in-one iron dominated magnet. To ensure that the two beam trajectories have the same path length, the beams will need to be crossed several times about the circumference. Space and optical designs must be incorporated into the layout to accommodate this feature. The two beams of the high field collider are naturally separated vertically in the common coil arc magnets.

\subsubsection{Design Approach}

To allow for lattice and component designs to progress simultaneously for both the LF and HF colliders, a general layout composed of basic modules was developed. As indicated in Figure 1, each collider is made up of two major arcs which connect two clusters of straight sections. The clusters contain space for two nearby interaction regions surrounded by two utility regions which are used for injection and extraction, accelerating cavities, etc. Shorter bending regions (DM, DM8) are located between each IR and UT region to allow collision debris and muon vectors to miss the downstream detector and utility enclosures. At the outset, only one cluster region - located at or near the Fermilab site - will be equipped with full IR and UT optics. The other cluster region will have the same straight section lengths, but optically will consist of simpler, FODO-type modules where applicable, with the exception of a special straight section design to accommodate future beam transfers between the LF and HF accelerators. More detailed descriptions of the lattices can be found in Chapters 2 and 3.

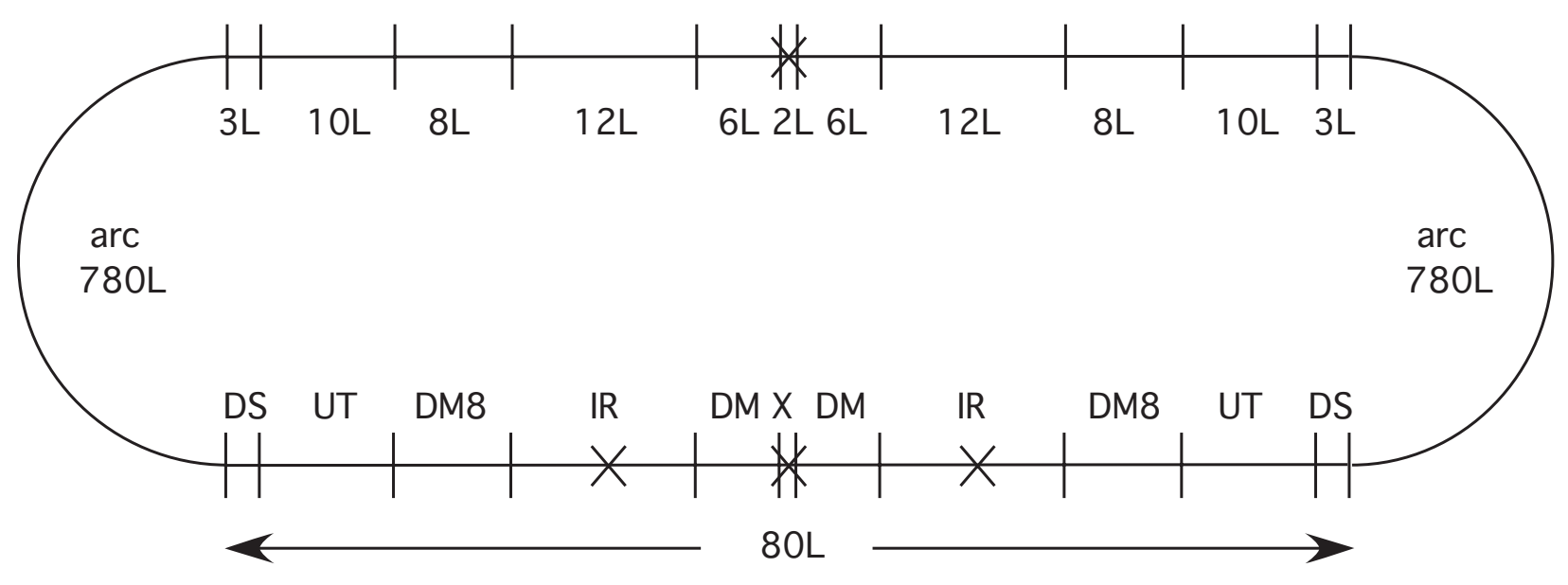

Figure 1: Schematic layout of collider modules. The figure is not to scale, emphasizing the straight section functions.

Historically, the half cell length of high energy synchrotrons has scaled roughly with the square root of energy. The space for injection/extraction devices and the space for final focusing at the Interaction Points (IPs) scale roughly equivalently. Thus, it was decided to make each of the special modules of the collider - interaction regions, utility regions, dispersion suppressors to have overall lengths equal to an integer number of standard arc half-cells, a design feature also adopted for the SSC project. By scaling the Tevatron half cell length to the LF energy, the distance between quadrupoles in the arcs of the new colliders would be approximately $135 \mathrm{~m}$. The HF ring could probably tolerate longer cells, but stronger focusing will be adopted to be consistent with the LF ring. The exact length of the standard cell will be determined by the chosen bunch spacing. To ensure that bunches will collide at any detector in the design, regardless of what modules exist between interaction points, the ring module lengths are designed in units of the bunch spacing.

Dispersion suppressor modules are adopted following the SSC design, which was also later used in the Fermilab Main Injector. A cell phase advance of 90 degrees has been chosen, for which a dispersion suppressor can be made using four special cells, each $3 / 4$ the length of a standard cell and each containing $2 / 3$ the bending of a standard cell. These modules reduce the periodic arc dispersion function to zero at the output of four such cells. The short bending regions between interaction regions and utility regions will be composed of back-to-back dispersion suppressor modules. If additional bending is required in these regions, it can be generated by inserting standard half cells between the DS modules. 


\subsubsection{Tunnel Considerations}

A configuration with two rings in one tunnel certainly places demands on tunnel diameter. The Fermilab Main Ring and Tevatron synchrotrons occupied a single $10 \mathrm{ft}$ diameter tunnel. The LEP tunnel at CERN was designed for two rings (though it will only have one) and has a $12 \mathrm{ft}$ diameter. The SSC design, which had two separate single-cold-mass magnets, one above the other, used a $12 \mathrm{ft}$ diameter tunnel. For this design, we will assume a $12 \mathrm{ft}$ diameter tunnel as well.

The LF ring is envisioned to lie near the floor of the $12 \mathrm{ft}$ diameter tunnel. The HF ring will have magnets of larger cross section, and thus will be located on magnet stands above the LF ring where the tunnel is wider. A cartoon view of the tunnel cross section is shown in Figure 2. The HF magnet design uses two vertically separated beams. The mid-point of the high field beams will be about $1 \mathrm{~m}$ above the mid-point of the two low field beams.

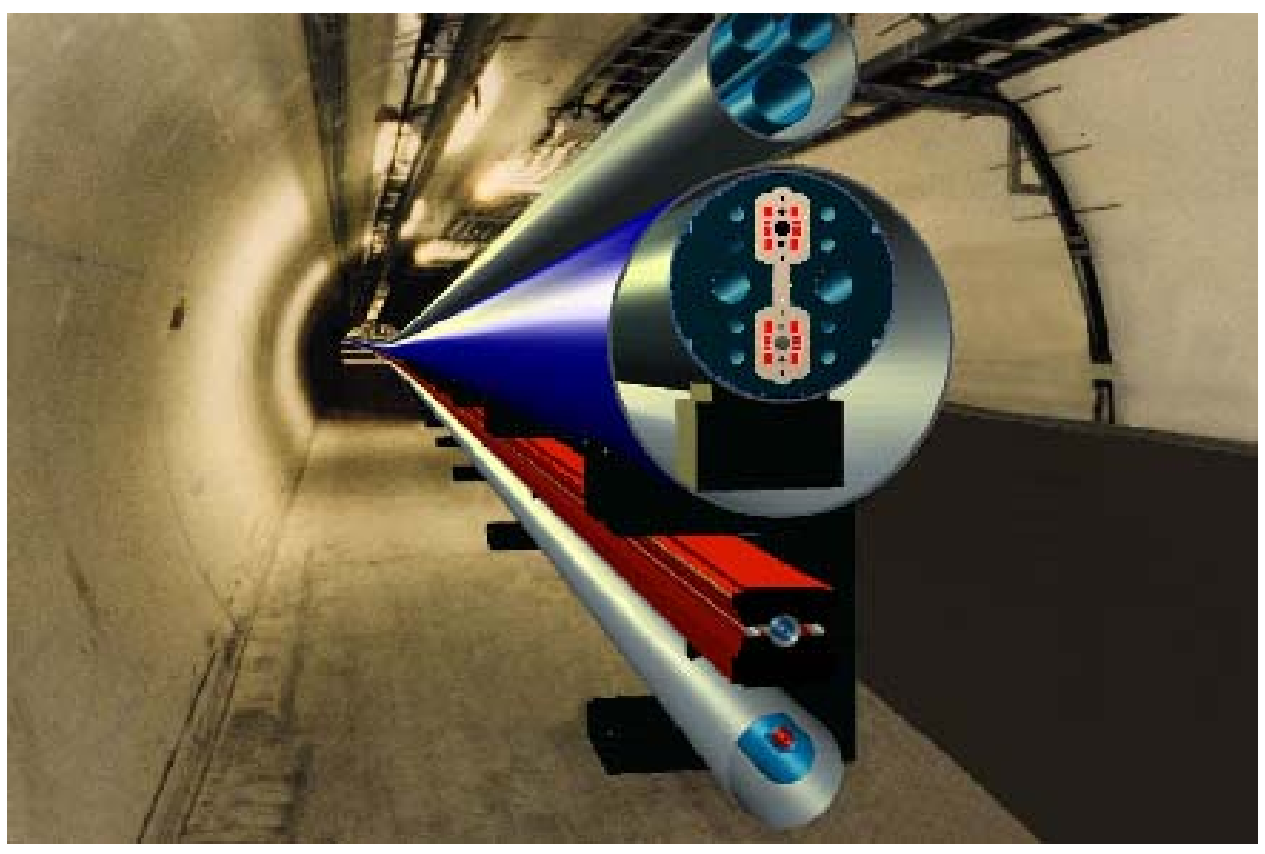

Figure 2: Cartoon drawing of the tunnel cross section in the VLHC collider arc, showing the low field ring with horizontally separated beams below the high field ring with vertically separated beams.

\subsection{Footprint and Optical Modules}

Table 1 lists the fundamental parameters of the VLHC footprint, as used in the Design Study [1]. In the transition from Stage 1 to Stage 2 operation, the experiments will remain centered on the same interaction points. (At the same time it is envisaged that they will be upgraded to take up more space along the beam line, increasing the distance from the IP to the first magnet, $L^{*}$, from 20 to $30 \mathrm{~m}$.) It is therefore necessary to allow for a bypass to keep the low field ring beams well clear of the experiments . This must be done without changing the total low field ring circumference. Figure 3 is a cartoon showing the layout of the interaction and utility regions on the Fermilab site, including a bypass with an approximate length of $8 \mathrm{~km}$.

Figure 4 shows, to scale, the same layout of the on-site optical modules, without the bypass, but including the exact location of the 2 interaction points (IPs). Injection from the Tevatron is away from the 2 IPs, since the VLHC is about 100 meters below the ground surface. One of the two abort lines per ring is shown, missing the neighboring IP by about 40 meters. A common abort dump area is possible for the four beams, approximately 100 meters from the horizontal crossing point " $\mathrm{X}$ " of the low field ring. Figure 4 also shows that the muon plume emanation from one IP misses the other experiment by approximately 70 meters.

Both low field and high field ring lattices are constructed from optical modules placed end to end like lego blocks. Each optical module - an arc, dispersion suppressor, interaction region straight, et cetera - has an overall length which is an integer multiple of $L_{h c}$, the arc half cell length. Low and high field modules with the same function can be quite different in implementation. For example, the low field arc half cell contains 2 combined function bending magnets, while the high field arc half 


\begin{tabular}{|lrl|} 
Circumference, $C$ & 233.037 & $\mathrm{~km}$ \\
Average arc radius, $R$ & 35.0 & $\mathrm{~km}$ \\
Number of interaction points & 2 & \\
Half cell length, $L_{h c}$ & 135.486 & $\mathrm{~m}$ \\
Half cell bend angle, $\theta_{h c}$ & 3.875 & $\mathrm{mrad}$ \\
Half cell count & 1720 & \\
Half cell harmonic, $n_{\lambda}$ & 24 & \\
Bunch spacing $(53.1 \mathrm{MHz}), S_{B}$ & 5.645 & $\mathrm{~m}$ \\
& 18.8 & $\mathrm{~ns}$ \\
Number of buckets & 41280 & \\
Phase advance per cell & 90.0 & $\mathrm{deg}$ \\
Revolution frequency & 1.286 & $\mathrm{kHz}$ \\
Revolution period, $T$ & .778 & $\mathrm{~ms}$ \\
Harmonic number, $h$ & 371520 & \\
RF frequency $(9 \times 53.1)$ & 478.0 & $\mathrm{MHz}$ \\
& & \\
\hline
\end{tabular}

Table 1: Fundamental lattice parameters common to both low and high field rings.

cell contains 7 pure dipoles. Nonetheless, optical modules in both low field and high field rings always have the same overall length and the same bending angle. For example, in both low and high field rings a dispersion Suppressor module is $3 L_{h c}$ long, constructed from 4 dispersions suppressor half cells of length $3 / 4 L_{h c}$, each with $2 / 3$ of the bending of a regular arc half cell.

Table 2 lists the sequence of optical modules in the region on the Fermilab site region in detail, and introduces short aliases such as "IR" for the Interaction Region modules, "DM" for the Dispersion Modules and "IA" for the Injection/Abort straight. The footprint geometry for the off-site region half way around the VLHC is identical. These two regions are separated by identical arcs.

One advantage of modular construction is that low and high field lattices are guaranteed to have almost identical footprints, and therefore to fit in the same tunnel, so long as corresponding low and high field modules are placed on top of each other. Figure 5 shows the transverse offset of the high field ring relative to the low field ring. The maximum deviation is only about $6 \mathrm{~mm}$, easily allowing one ring to be placed on top of the other at all locations in the VLHC tunnel.

\subsubsection{Half cell length}

The average bending radius of the arcs is made as close as possible to the goal value of $R=35 \mathrm{~km}$ by adjusting the total equivalent number of (bending) arc cells in each ring. Each of the 4 short half cells in each dispersion suppressor has $2 / 3$ the bending of an arc half cell. Thus, the 20 dispersion suppressors (on either side of 10 straights) have a total bending equivalent

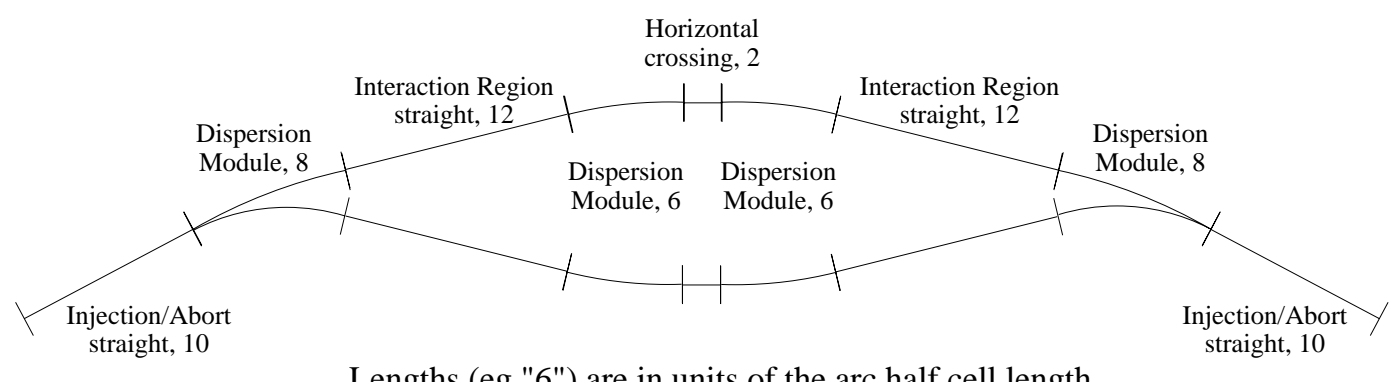

Figure 3: Interaction and utility regions on the Fermilab site, including a bypass to transport low field ring beams past the experiments in Stage 2 operation. 


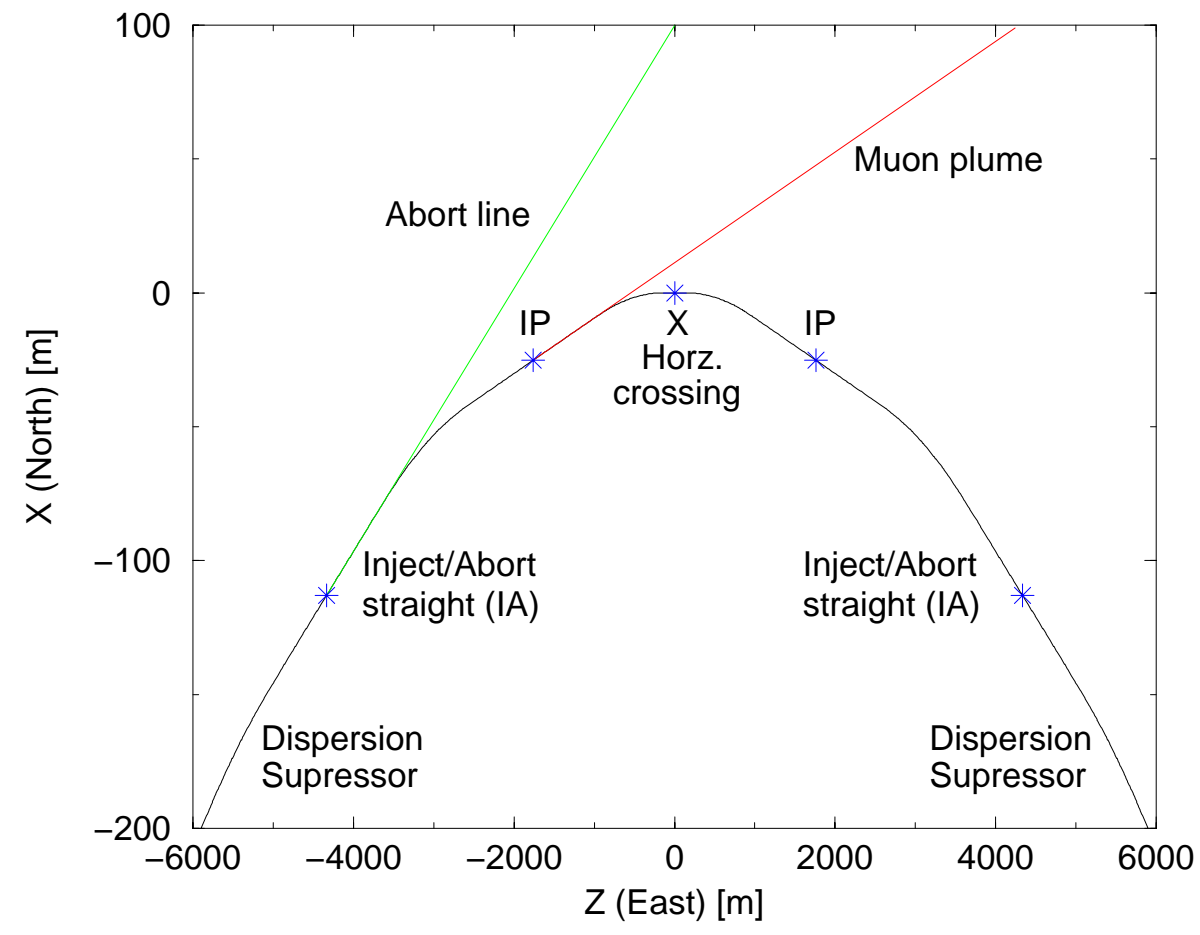

Figure 4: Scale layout of the on-site cluster region of the VLHC, showing the clearance of abort lines and muon plumes from the experiments, and suggesting the use of a common abort dump area.

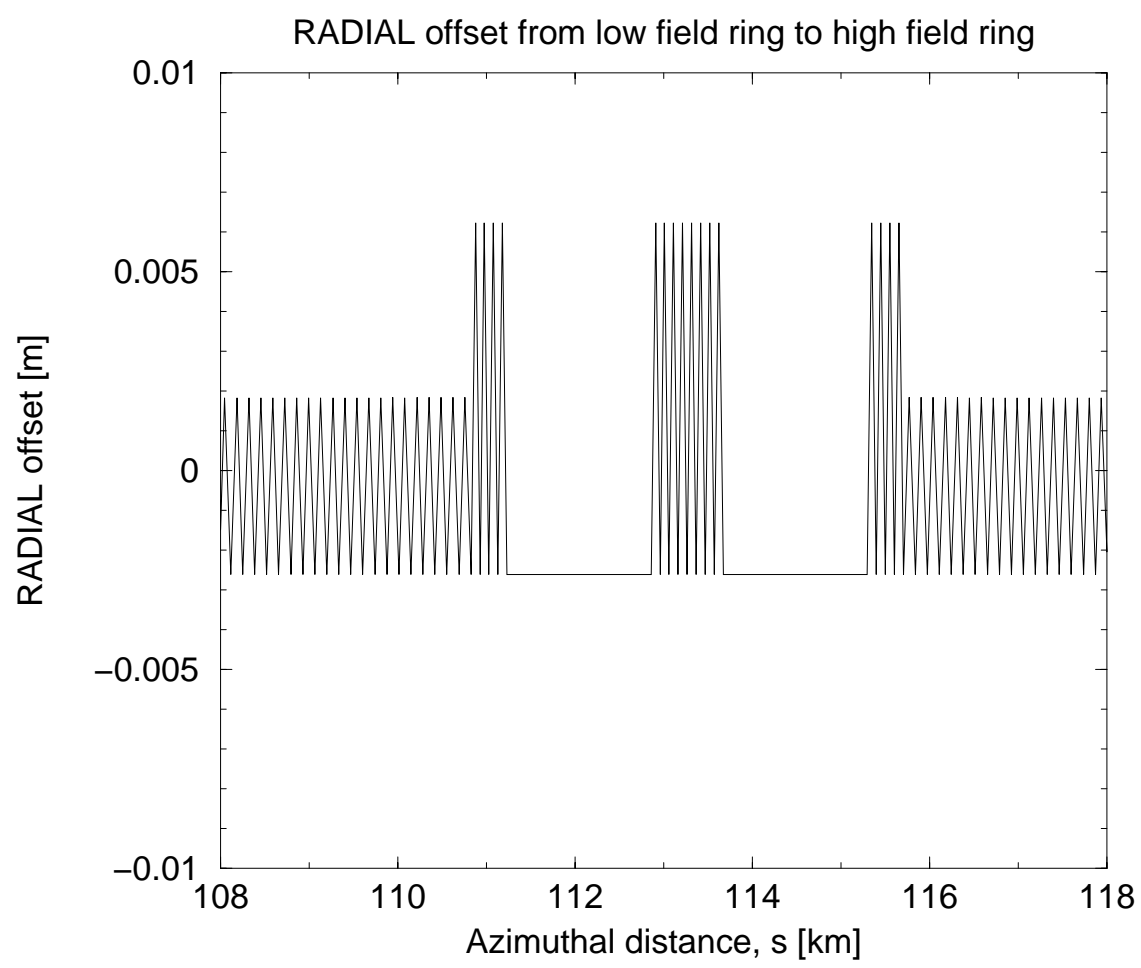

Figure 5: The transverse offset of the high field ring, relative to the low field ring. Both rings easily fit in the same tunnel. 
to $160 / 3$ arc half cells. If there are $N=784$ arc half cells in each half of the VLHC, then the bend per half cell is

$$
\theta_{h c}=\frac{2 \pi}{2 N+160 / 3}=3.875[\mathrm{mrad}]
$$

and the average arc bend radius is

$$
R=\frac{L_{h c}}{\theta_{h c}}=34.961[\mathrm{~km}]
$$

Is the nominal arc half cell length of $L_{h c}=135.4865 \mathrm{~m}$ close to an optimum? What other lengths are possible?

\begin{tabular}{|llrl|}
\hline & ALIAS & $L / L_{h c}$ & COMMENT \\
MODULE & & & \\
Dispersion Suppressor & DS & 3 & \\
Injection/Abort straight & IA & 10 & \\
Dispersion Module & DM8 & 8 & contains 2 arc half cells \\
Interaction Region & IR & 12 & \\
Dispersion Module & DM & 6 & \\
Crossing "straight" & X & 2 & \\
Dispersion Module & DM & 6 & \\
Interaction Region & IR & 12 & \\
Dispersion Module & DM8 & 8 & contains 2 arc half cells \\
Injection/Abort straight & IA & 10 & \\
Dispersion Suppressor & DS & 3 & \\
& & & \\
Utility total & & 80 & \\
Arc & & 780 & \\
GRAND TOTAL & & 1720 & 2 arcs plus 2 utilities \\
& & & \\
\hline
\end{tabular}

Table 2: Sequence of optical modules in the on-site cluster region. There is another region of identical footprint geometry on

\begin{tabular}{|c|c|c|c|}
\hline$n_{\lambda}$ & $L_{h c}[\mathrm{~m}]$ & & COMMON FACTORS \\
\hline 18 & 101.6149 & $2 * 3 * 3$ & 236918 \\
\hline 20 & 112.9054 & $2 * 2 * 5$ & 2451020 \\
\hline 22 & 124.1959 & $2 * 11$ & 21122 \\
\hline 24 & 135.4865 & $2 * 2 * 2 * 3$ & 234681224 \\
\hline 26 & 146.7770 & $2 * 13$ & 21326 \\
\hline 28 & 158.0676 & $2 * 2 * 7$ & 2471428 \\
\hline 30 & 169.3581 & $2 * 3 * 5$ & 2356101530 \\
\hline 32 & 180.6486 & $2 * 2 * 2 * 2 * 2$ & 2481632 \\
\hline 34 & 191.9392 & $2 * 17$ & 21734 \\
\hline
\end{tabular}
the far side of the ring. The two clusters are separated by two arcs, each 780 half cells long.

Table 3: Potential arc half cell harmonics and lengths. The bunch spacing can trivially be multiplied from its nominal value by any of the common factors of the "half cell harmonic factor", $n_{\lambda}$.

An important advantage of modular design is the ability to independently modify one particular module without adjusting any other modules. For example, it is possible to increase or decrease the length of a module in quanta of $L_{h c}$ while (almost) trivially maintaining optical matching conditions. Such length modifications will not disturb the bunch spacing - or the injection scheme or the RF system, et cetera - if the arc half cell length is an integer times the minimum allowed bunch spacing. In this 
case the total circumference is guaranteed to correspond to an integer number of evenly spaced bunches. The (minimum) bunch spacing $S_{B}$ is taken to be one 53.1 MHz RF wavelength in the Tevatron

$$
S_{B}=\lambda_{53} \equiv \frac{2,000 \pi}{1113}=5.645 \text { meters }
$$

This guarantees that the filling schemes from the Tevatron will be relatively simple. The bunch time spacing of about 18.8 ns is not unreasonably challenging to the experiments.

Thus the half cell length is

$$
L_{h c}=n_{\lambda} S_{B}
$$

The bunch spacing can trivially be increased (for example during commissioning) by any of the common factors of the "half cell harmonic number" $n_{\lambda}$. Table 3 shows all the even values of $n_{\lambda}$ in the half cell length range from 100 meters to 200 meters. The value of $n_{\lambda}=24$ was chosen for both low and high field rings in the Design Study [1], corresponding to a half cell length of $L_{h c}=135.4865$ meters.

This half cell length is short enough to keep the natural horizontal emittance due to synchrotron radiation in the high field ring, $\epsilon_{\text {nat }}=0.04 \mu \mathrm{m}$, much smaller than the horizontal emittance $\epsilon_{x} \approx 0.20 \mu \mathrm{m}$ which must be maintained (by heating) to avoid beam-beam limits in the store. Figure 6 shows the cubic dependence of the natural emittance on the half cell length under the reasonable approximation of a purely FODO cell lattice with thin quadrupoles and 100\% dipole packing fraction [3, 4].

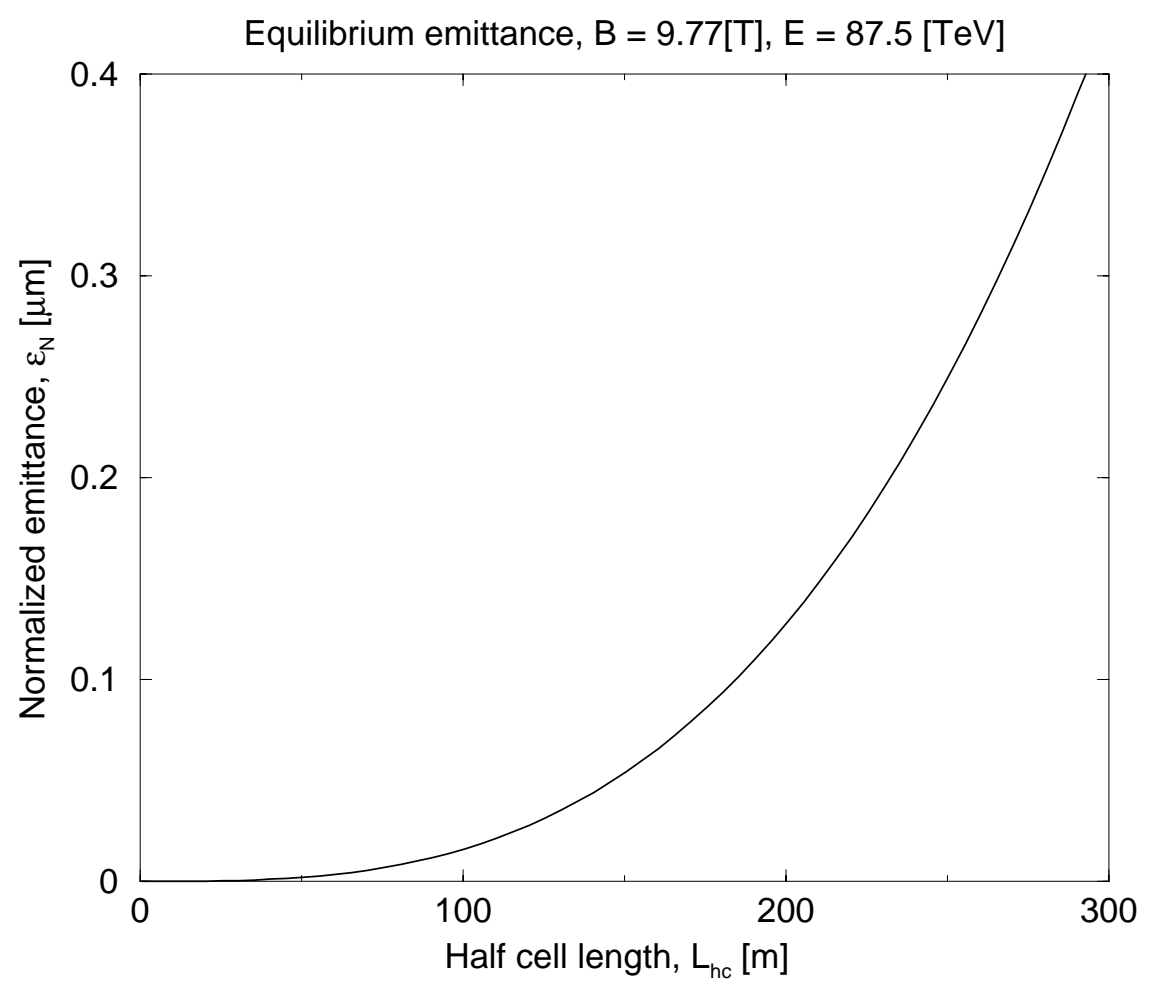

Figure 6: Natural emittance from synchrotron radiation versus arc half cell length in the high field ring.

A stronger limit to the maximum arc half cell length is the need to limit the vulnerability of the low and high field rings to systematic field harmonic errors in the arc bending magnets. This is more critical for the low field ring, simply because the injection energy is an order of magnitude lower ( $1 \mathrm{TeV})$ than for the high field ring (10 TeV). A crude calculation [5] shows that the systematic harmonic $b_{n}$ must obey

$$
\frac{b_{n}}{r_{0}^{n}} \leq \widehat{\Delta Q_{x}} \frac{1}{D_{n}} L_{h c}^{-(n+1) / 2}\left(\frac{\beta \gamma}{m^{2} \epsilon_{x}}\right)^{(n-1) / 2}
$$

where $r_{0}=10 \mathrm{~mm}$ is the reference radius, $\widehat{\Delta Q_{x}}$ is the maximum allowed tune shift, $D_{n}$ is a numerical coefficient (see Table 4), and $m$ is both the horizontal betatron amplitude in units of $\sigma_{x}$ and the constant momentum offset in units of $\sigma_{p} / p$. 
Equation 5 assumes fully packed FODO cells with thin quads, and assumes that the betatron beam size at an F quad is equal to the momentum spread size [5]. Although crude, Eqn. 5 correctly shows the scaling with respect to injection energy and cell length. Tracking studies are necessary when increased accuracy is required. Figure 7 illustrates how the sensitivity to systematic harmonics depends on the half cell length according to Eqn. 5, in the low and high field rings respectively.

In conclusion, the current value of $L_{h c}=135.4865 \mathrm{~m}$ is by no means fully optimized, not least because we do not accurately understand what values of systematic magnet harmonics might be achieved in industrial production of the arc bending magnets.

\begin{tabular}{|llc|}
\hline $\mathrm{n}$ & Multipole & $D_{n}$ \\
& & \\
1 & Quadrupole & .8333 \\
2 & Sextupole & 2.412 \\
3 & Octupole & 6.712 \\
4 & Decapole & 19.18 \\
5 & 12-pole & 56.49 \\
6 & 14-pole & 170.9 \\
& & \\
\hline
\end{tabular}

Table 4: Lowest order $D_{n}$ values, with a phase advance of $\phi_{c}=90$ degrees per FODO cell. 
$1 \mathrm{TeV}$
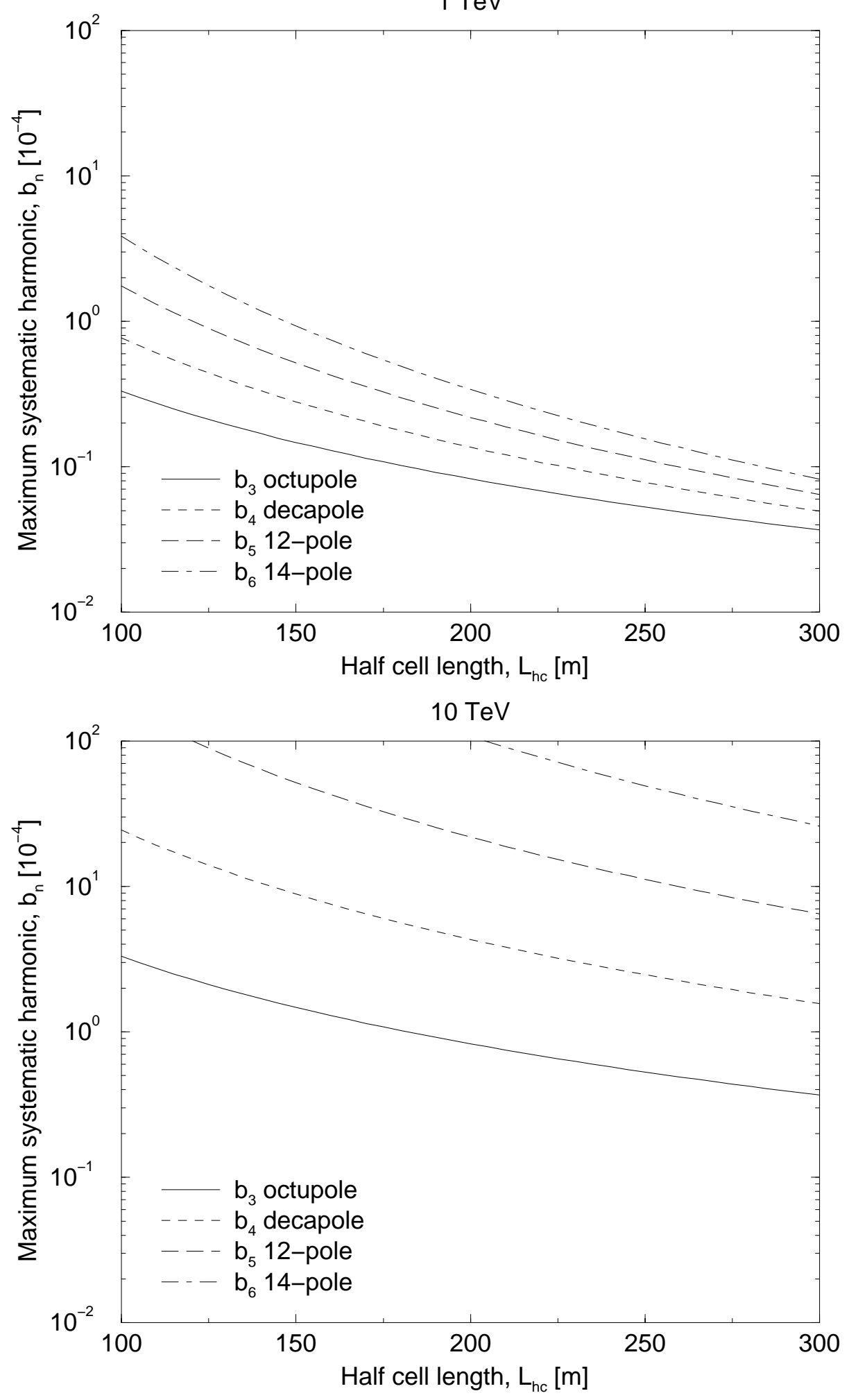

Figure 7: Maximum allowed systematic harmonics at an injection energy of $1 \mathrm{TeV}$ for the low field ring (TOP) and $10 \mathrm{TeV}$ for the high field ring (BOTTOM), versus half cell length. A maximum tune shift of $\Delta Q=0.05$ is permitted with an emittance of $\epsilon=1.5[\mu \mathrm{m}]$, a betatron amplitude of $4 \sigma$, and a constant momentum offset of $\Delta p / p= \pm 4 \sigma_{p} / p$. 


\section{STAGE 1: THE LOW-FIELD RING}

The first stage low-field collider will have a top energy of $20 \mathrm{TeV}$ and peak (initial) luminosity of $10^{34} \mathrm{~cm}^{-2} \mathrm{sec}^{-1}$. The collider will use the Tevatron as its injector operating at a transfer energy of $900 \mathrm{GeV}$. For this study a transverse emittance of $1.5 \pi \mathrm{mm} \mathrm{mrad}$ (rms, normalized) is used, which is typical for the Fermilab Booster, though about half the value at the Tevatron under recent normal operations. It is anticipated that with further Run II experience, the efficiency of emittance preservation will increase. Utilizing the present acceleration systems of the Tevatron injector chain, the collider has a bunch spacing of $5.645 \mathrm{~m}$ (53.1 MHz) which sets the number of available RF buckets. With a 90\% filling fraction to allow for gaps in the beam for various kicker rise times, the number of protons per bunch required in the Stage 1 collider is approximately $2.6 \times 10^{10}$, similar to Tevatron bunch intensities during previous Fixed Target operations. Using an interaction region design with a $\beta^{*}=0.3 \mathrm{~m}$, we arrive at the desired initial luminosity. Table 5 lists the general parameters of the Stage 1 collider.

Luminosity, bunch intensity versus time

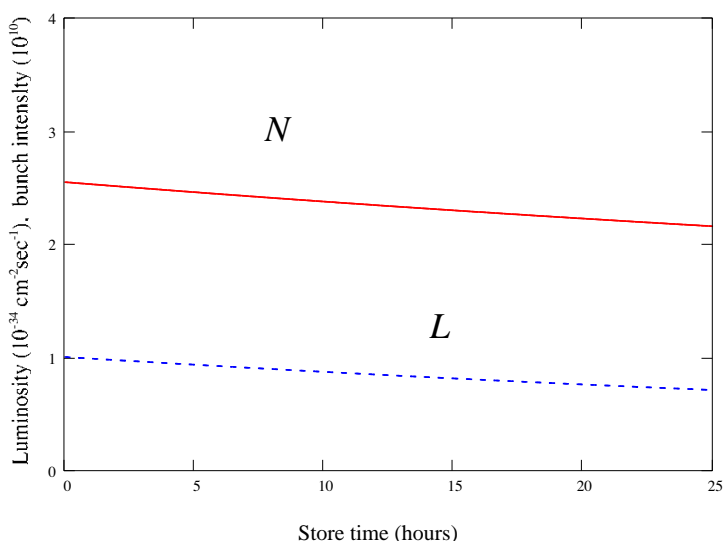

Emittance evolution versus time

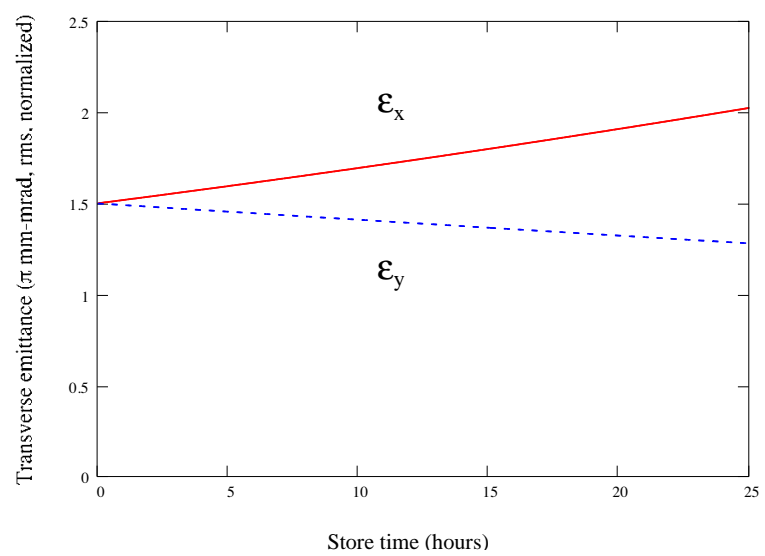

Figure 8: Evolution of collider parameters during a typical store.

Typical store parameter evolution is depicted in Figure 8. The figure shows the proton bunch intensity and luminosity decreasing due to collisions. The vertical emittance also decreases slightly due to synchrotron radiation damping which occurs with a damping time of about $100 \mathrm{hr}$. Due to the use of gradient magnets in the Stage 1 collider the horizontal emittance actually will be anti-damped, and will increase at approximately the same rate. This should not be problematic since store times will be much less than 100 hours. While horizontal damping can be restored by adjusting the lengths and strengths of the gradient magnets, the required changes in bend radius for the $\mathrm{F}$ and $\mathrm{D}$ magnets are approximately $\pm 10 \%$. 
Storage energy

Peak luminosity

Number of interaction points

Circumference

Arc average radius

Packing fraction

Injection energy

Transverse normalized emittance, $r m s(H \& V$, inject)

Initial bunch intensity

Number of bunches (90\% fill fraction)

Total protons per beam

Average beam current

Stored energy per beam at collision

Bend field at storage

Bend magnet gradient

Maximum arc beta

Maximum arc dispersion

Phase advance per cell

Betatron tunes $(\mathrm{H}, \mathrm{V})$

Transition gamma

Max RMS arc beam size (inject)

Bunch spacing $(53.1 \mathrm{MHz})$

Bunch length

Longitudinal emittance, rms (inject)

$\mathrm{RF}$ voltage at storage

Fill time

Acceleration time

Beam size (rms) at IP (storage)

Total crossing angle (10 $\sigma$ separation in drift space)

Distance from IP to first magnet

$\beta^{*}$ at IP $(\mathrm{H} \& \mathrm{~V})$

Maximum interactions per crossing

Debris power at IP (each direction)

SYNCHROTRON RADIATION AT STORE

Energy loss per turn per particle

Radiation damping time $\left(\tau_{0}\right)$

(anti-damping in H plane)
$\mathrm{TeV}$

$10^{34} \mathrm{~cm}^{-2} \mathrm{~s}^{-1}$

2

$233.037 \mathrm{~km}$

$35.0 \mathrm{~km}$

$89 \%$

$0.9 \mathrm{TeV}$

$1.5 \mu \mathrm{m}$

$2.6 \times 10^{10}$

37152

$9.5 \times 10^{14}$

$195 \mathrm{~mA}$

3.0 GJ

$2.0 \mathrm{~T}$

$9.0 \mathrm{~T} / \mathrm{m}$

$411 \mathrm{~m}$

$1.56 \mathrm{~m}$

$90.0 \mathrm{deg}$

$218.419,218.425$

192.1

$1.2 \mathrm{~mm}$

$5.645 \mathrm{~m}$

$18.8 \mathrm{nsec}$

$30 \mathrm{~mm}$

0.4 eV-s

50 MV

$60 \mathrm{~min}$

$1000 \mathrm{sec}$

$4.6 \mu \mathrm{m}$

$153 \mu \mathrm{rad}$

$21 \mathrm{~m}$

$30 \mathrm{~cm}$

20

$3 \mathrm{~kW}$

$38 \mathrm{keV}$

$100 \mathrm{hr}$

Table 5: Nominal store parameters for the low field collider. 


\subsection{Lattice}

The bulk of the lattice is characterized by its construction from just three optical building blocks - arc, dispersion suppressing, and straight-section cells. Unlike the HF ring, the LF ring arc and suppressor cells are constructed from combined function magnets. Parameters describing these standard modules appear in Table 6. (The LF and HF ring geometries are detailed elsewhere). The arc and suppressor cells have space allocated at maximum- $\beta$ sites for 4 correction elements. Additional corrector space is available at the quarter-cell locations $\left(\beta_{x}=\beta_{y}\right)$ for lumped correction of systematic multipoles.[6]

\begin{tabular}{|c||c|c||c|c|c|c|}
\hline Cell Type & Cell Length $(\mathrm{m})$ & Magnet Type & $\mathrm{L}_{\text {magnetic }}(\mathrm{m})$ & $\# /$ cell & B (T) & B' (T/m) \\
\hline Arc & 270.973 & GF / GD & 65.75 & 4 & 1.966 & \pm 9.278 \\
Suppressor & 203.230 & GSF / GSD & 48.81 & 4 & 1.766 & \pm 16.687 \\
Straight & 270.973 & QF / QD & 6.10 & 4 & 0 & \pm 69.333 \\
\hline
\end{tabular}

Table 6: Cell and magnet parameters at $20 \mathrm{TeV}$ in the standard LF optical units. With $90^{\circ}$ of phase advance per cell, $\beta_{\max }=411 \mathrm{~m}$, and $\eta_{\max }=1.56 \mathrm{~m}$.

Figure 9 illustrates the lattice functions at injection through the single cluster of specialized straight sections in the ring. Two utility straights, each of which must accommodate $900 \mathrm{GeV}$ injection from the Tevatron plus abort of $20 \mathrm{TeV}$ beams are shown. The beams collide with a horizontal crossing angle at two interaction points where $\beta_{\text {max }}=30 \mathrm{~cm}$. In a special cell, midway between the IPs, the beams are made to cross over between the inner and outer horizontal apertures. Diametrically opposed in the ring, a mirror of this cell is installed to ensure identical path lengths for the two circulating beams.

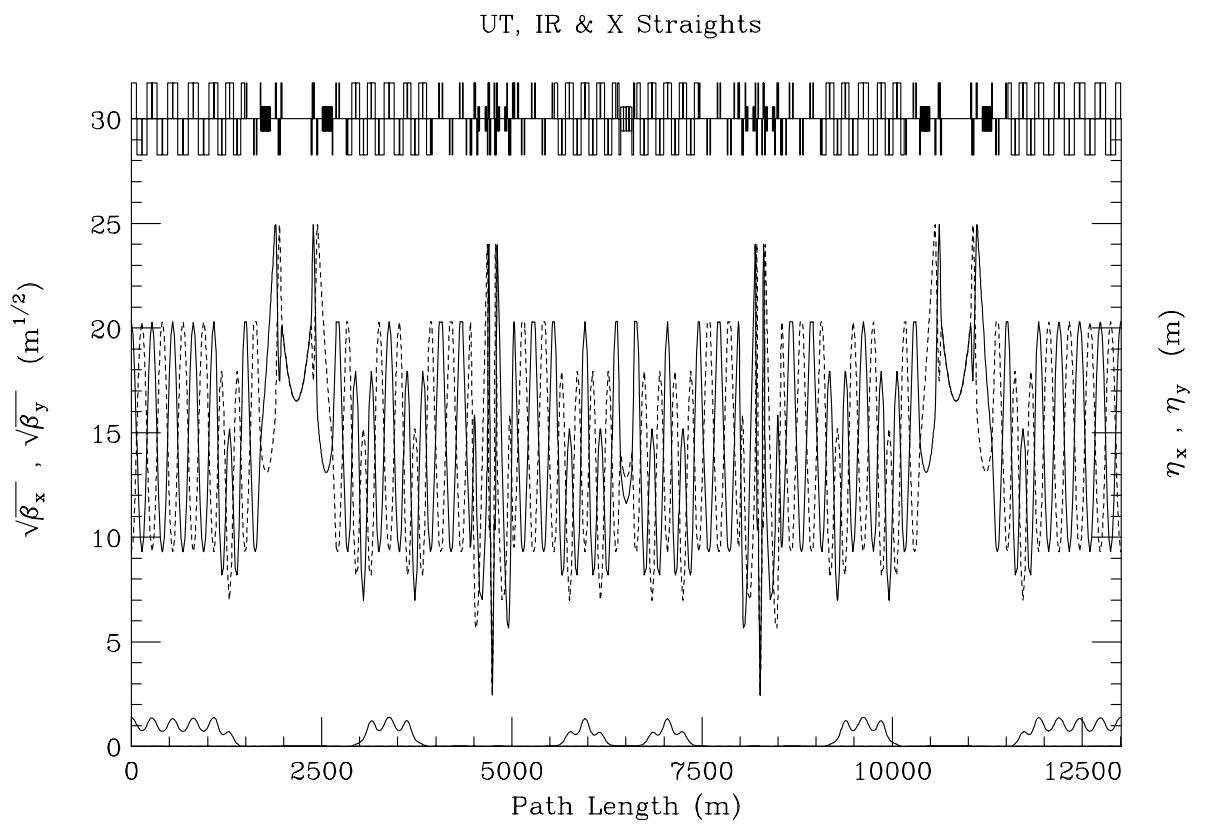

Figure 9: Injection lattice functions through the cluster region containing the interaction regions, utility straights, and a single cell (center) where the beams cross between the inner and outer apertures.

\subsubsection{Interaction Regions}

The final foci in the IRs are anti-symmetric triplets, formed from single-bore, $300 \mathrm{~T} / \mathrm{m}$ magnets. Four additional quadrupole circuits, comprising double-bore, $70 \mathrm{~T} / \mathrm{m}$ magnets, are also used in optical matching. With a total of 6 independently-tunable quadrupole circuits available it is possible to match the four $\beta$ 's and $\alpha$ 's from the IP to regular FODO cells, plus hold the phase 
advance $\Delta \mu$ constant across the IR through the squeeze from $\beta^{*}=6.00 \mathrm{~m} \rightarrow 0.30 \mathrm{~m}$. Fixing $\Delta \mu$ eliminates the necessity of a special phase trombone to maintain the nominal operating point. Figure 10 shows the lattice functions through the IR during collisions, and Table 7 lists quadrupole gradients both at injection and collision.

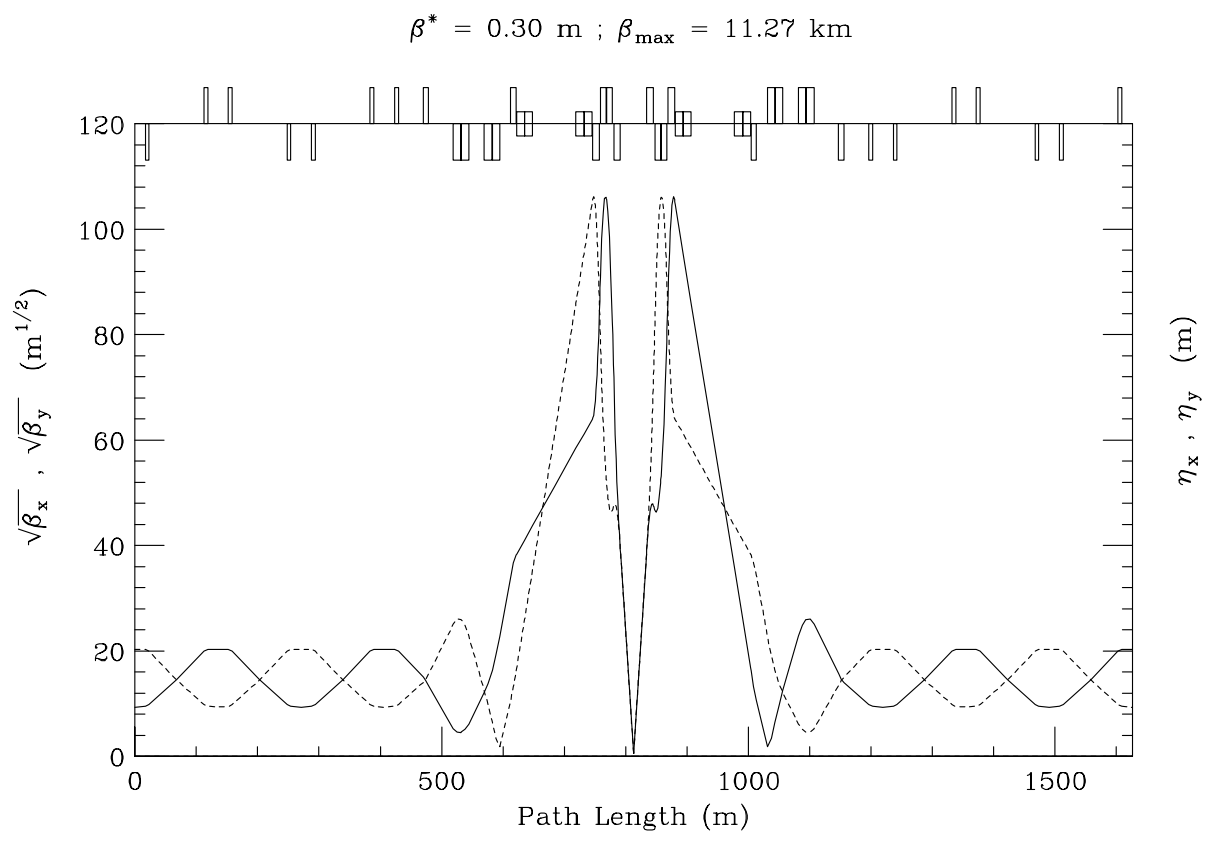

Figure 10: IR lattice functions in the collision configuration.

\begin{tabular}{|c||c|c|c|}
\hline Quad \# & $\begin{array}{c}\mathrm{L}_{\text {magnetic }} \\
(\mathrm{m})\end{array}$ & $\begin{array}{c}\text { Gradients }(\mathrm{T} / \mathrm{m}) \\
\beta^{*}=6.00 \mathrm{~m} \\
{\left[\beta_{\max }=575 \mathrm{~m}\right]}\end{array}$ & $\begin{array}{c}\text { Gradients }(\mathrm{T} / \mathrm{m}) \\
\beta^{*}=0.30 \mathrm{~m} \\
{\left[\beta_{\max }=11.3 \mathrm{~km}\right]}\end{array}$ \\
\hline 1 & 10.90 & $\mp 301.5$ & $\mp 298.2$ \\
$2 \mathrm{a}$ \& 2b & 9.22 & \pm 304.3 & \pm 294.5 \\
3 & 10.90 & $\mp 301.5$ & $\mp 298.2$ \\
4 & 12.19 & $\mp 51.11$ & \pm 62.40 \\
$\mathbf{5 a}$ \& 5b & 12.19 & \pm 69.44 & $\mp 65.51$ \\
6a \& 6b & 12.19 & $\mp 62.00$ & $\mp 65.51$ \\
7 & 7.62 & \pm 0.802 & \pm 66.69 \\
\hline
\end{tabular}

Table 7: IR gradients at $20 \mathrm{TeV}$ for injection $\left(\beta^{*}=6 \mathrm{~m}\right)$ and collision $\left(\beta^{*}=30 \mathrm{~cm}\right)$ optics. The phase advances across the IR are fixed at $\Delta \mu_{x}=\Delta \mu_{y}=2.250$ through the squeeze. Highlighted entries indicate quadrupoles that change polarity.

The circulating beams are separated horizontally by $15 \mathrm{~cm}$ throughout the arcs and $70 \mathrm{~T} / \mathrm{m}$ straight-section quads. Four dipoles, $13.6 \mathrm{~m}$ each at $1.97 \mathrm{~T}$, situated between the Q3 and Q4 quadrupoles bring the beams together at the entrance to the triplet for collisions at the IP. Dipoles downstream of the IP separate the beams again and channel them back into the inner and outer rings. A half-crossing angle of $77 \mu \mathrm{r}$ at the IP gives $10 \sigma$ separation between the beams at the first parasitic crossing $\left(\epsilon_{N}\right.$ $=1.5 \pi \mu \mathrm{m}$ at $20 \mathrm{TeV})$.

\subsubsection{Injection / Abort}

To accommodate $900 \mathrm{GeV}$ injection from the Tevatron, as well as $20 \mathrm{TeV}$ proton abort, 5 straight-section cells are modified as shown in Figure 11. A long drift space $(377 \mathrm{~m})$ is created by triplet focusing to produce sufficient room for abort Lambertsons 
(not shown) and beam extraction. Quadrupole properties are given in Table 8.

\begin{tabular}{|c|c|c|}
\hline Quad \# & $\mathrm{L}_{\text {magnetic }}(\mathrm{m})$ & Gradients $(\mathrm{T} / \mathrm{m})$ \\
\hline 1 & 15.18 & 44.57 \\
2a \& 2b & 14.27 & 44.57 \\
3 & 15.18 & 44.57 \\
4 & 5.47 & 69.33 \\
\hline
\end{tabular}

Table 8: Utility region gradients at $20 \mathrm{TeV}$ for matching between the long central drift and standard FODO parameters.

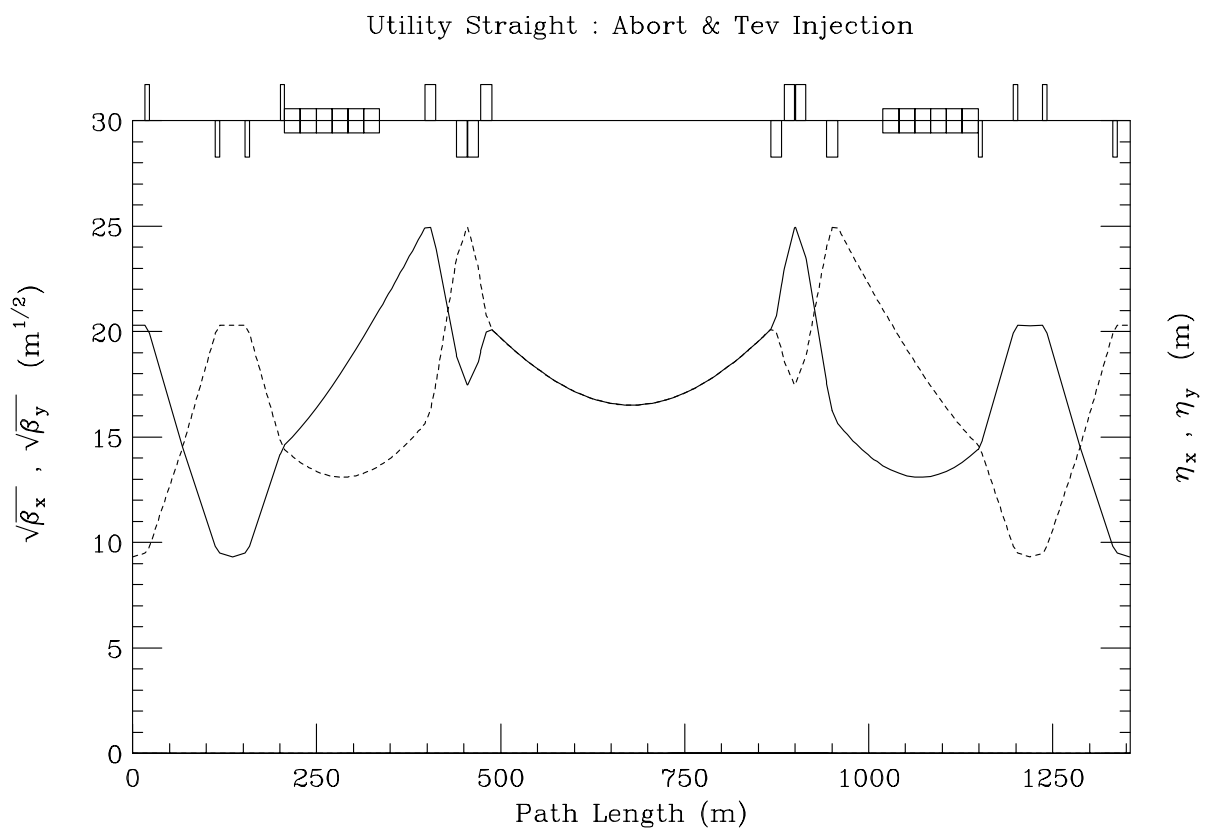

Figure 11: Layout of a utility straight supporting beam transfer from the Tevatron and $20 \mathrm{TeV}$ abort.

Dipoles separate the circulating beams through the UT straight to make room for RF cavities downstream of the second triplet. Three $21.54 \mathrm{~m}$ dipoles at $2 \mathrm{~T}$ bend the beams apart by $40 \mathrm{~cm}$, followed immediately by 3 more dipoles that flatten out the trajectories. This pattern is reversed downstream of the RF to bring the beams back into standard double-bore magnets.

\subsubsection{Beam Crossovers}

With the beams separated horizontally in the arcs it is necessary to move the beams between the inner and outer rings to maintain identical path lengths. In the LF ring 2 cross-over cells (Figure 12) are inserted on opposite sides of the ring. Four rolled dipoles move the beams the $15 \mathrm{~cm}$ from one channel to the other. Each dipole is $36 \mathrm{~m}$ at $1.944 \mathrm{~T}$. The first dipole is rolled by0.12 rad to start separating the beams vertically, while bringing them closer together horizontally. The second bend is rolled by -0.12 rad to flatten the vertical trajectory. At the crossing point the beams are separated vertically by 9 mm. With $\beta_{y}$ $=165 \mathrm{~m}$, and $\epsilon_{N}=1.5 \pi \mu \mathrm{m}$, this is $17.75 \sigma$ at the injection energy of $900 \mathrm{GeV}$. Rolled dipoles after the crossing point remove the vertical offsets and complete the $15 \mathrm{~cm}$ transverse transfer of beams to the opposite channel.

\subsubsection{Miscellaneous Insertions}

For momentum collimation a localized dispersion wave is generated in a 5-cell straight section (Figure 13). Four $45 \mathrm{~m}$ dipoles (2T @ $20 \mathrm{TeV}$ ) at the upstream end of the straight move the beams transversely by $0.36 \mathrm{~m}$, creating $\pm 70 \mathrm{~cm}$ of dispersion. Four 


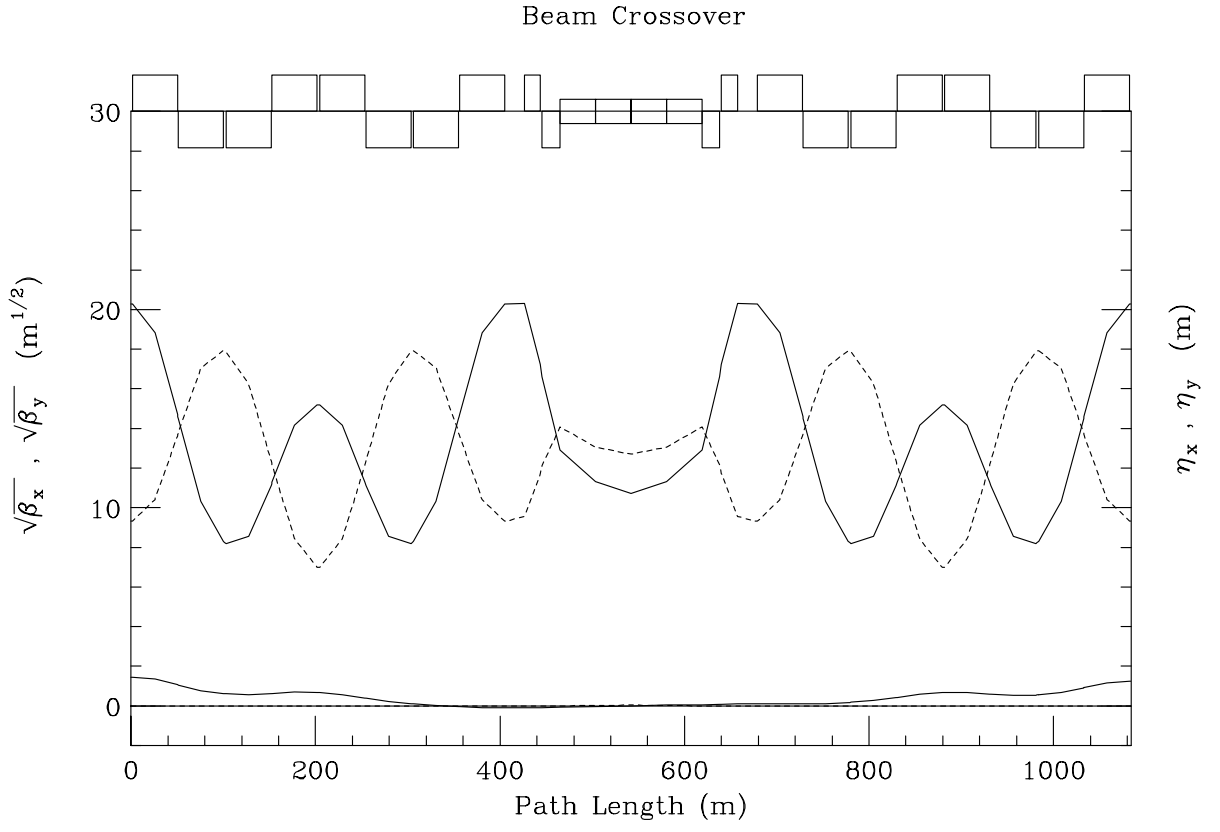

Figure 12: Cross-over cell with doublet focusing.

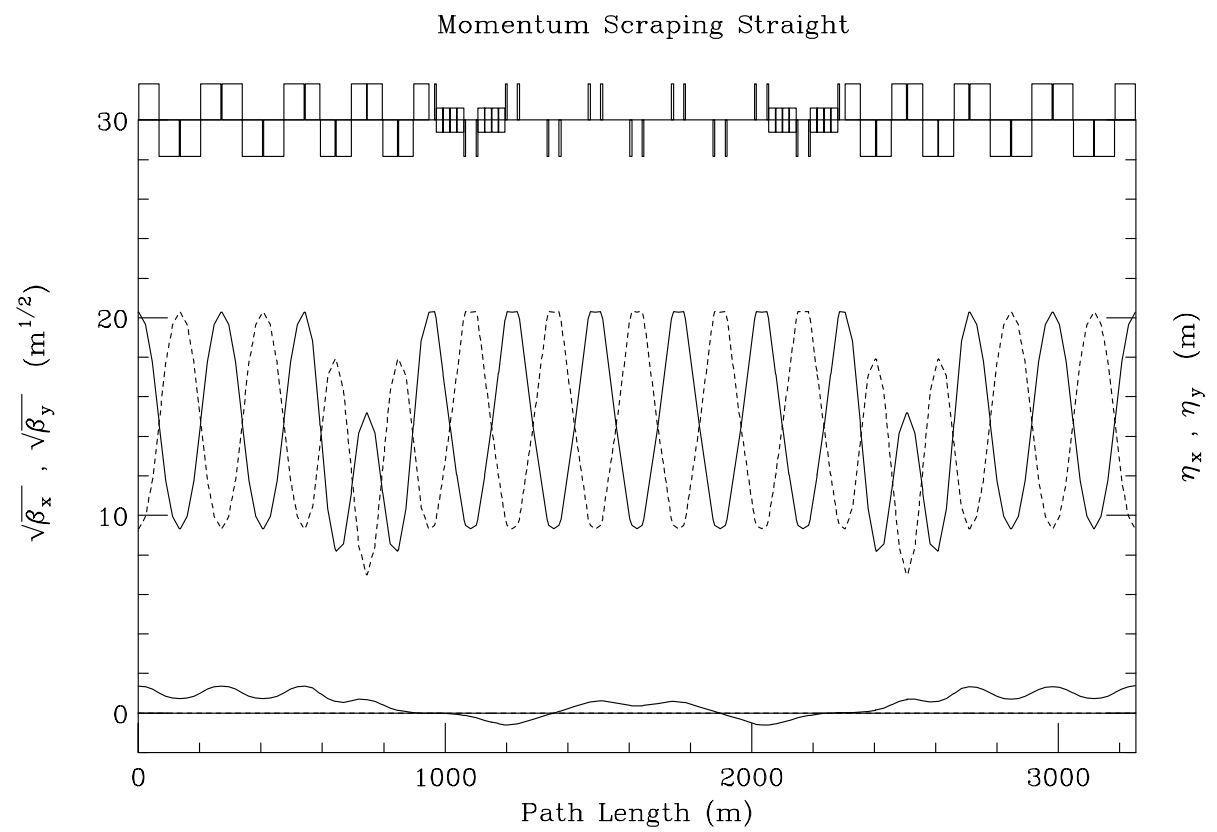

Figure 13: Dispersion creation in a 5-cell straight for collimation. 
more dipoles downstream bring the beams back onto their nominal trajectory and cancel the dispersion.

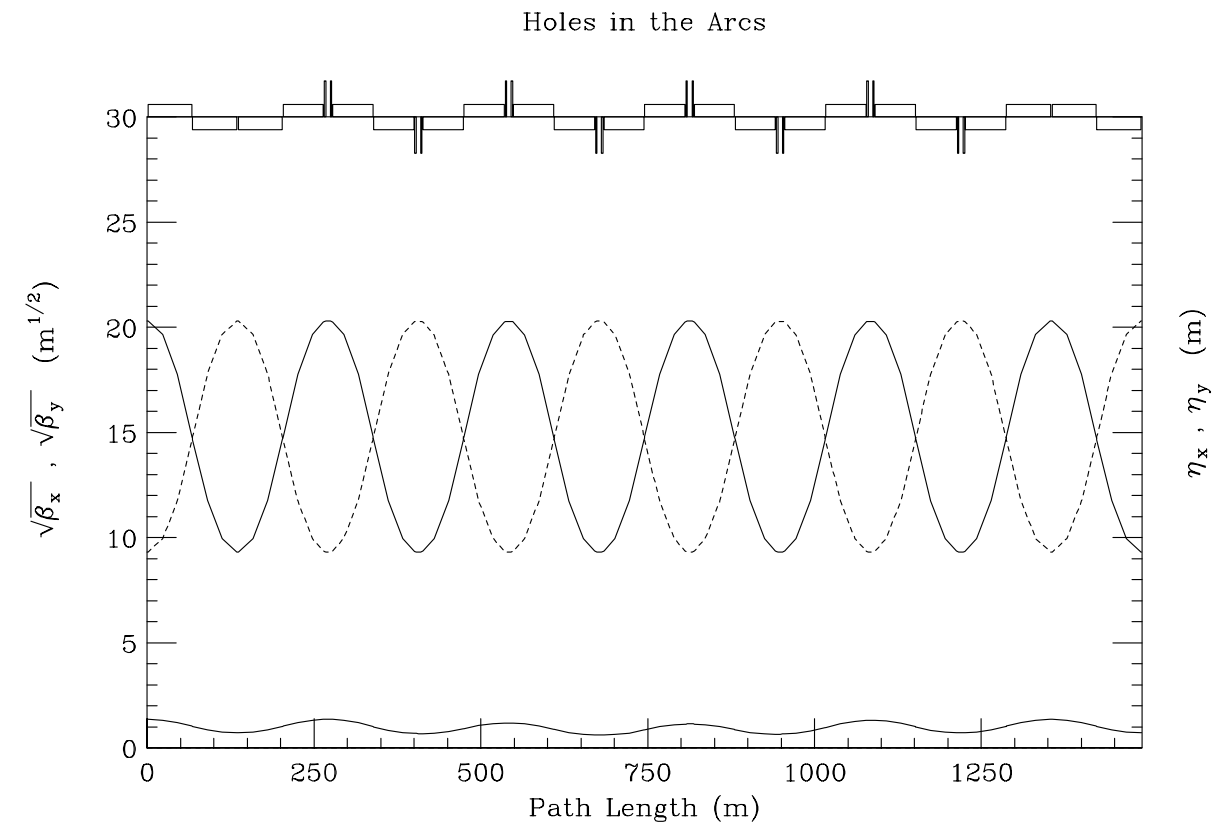

Figure 14: Achromatic generation of free space in the arcs for dampers, snakes, etc.

Free space can be created in the arcs by replacing standard arc gradient magnets with a shorter, modified version plus a small quadrupole. In the example illustrated by Figure 14, there is $7.0 \mathrm{~m}$ of space at each of the 8 locations indicated. Through this insertion, the $\beta$ 's, $\alpha$ 's, and phase advance of an arc cell are exactly reproduced by a gradient magnet with $B^{\prime}=9.185 \mathrm{~T} / \mathrm{m}$ ( $1 \%$ weaker than the regular arc magnets) plus a $2.0 \mathrm{~m}$ quadrupole with $B^{\prime}=26.14 \mathrm{~T} / \mathrm{m}$. The low field of the quadrupole makes it just a longer version of the normal arc trim quads. The dispersion wave created by the holes is canceled locally with $90{ }^{\circ}$ of phase advance per cell. 


\subsection{Dynamic Aperture and Field Quality}

The required dynamic aperture is determined by the available mechanical aperture. Collimators define the limiting aperture in most cases and they are usually placed at around $6 \sigma$ from the center of the beam pipe. This allows $3 \sigma$ for the beam distribution, and another $3 \sigma$ for injection errors and orbit drifts over time. The real dynamic aperture therefore must be greater than $6 \sigma$ in order to avoid significant losses of particles at places in the ring outside the beam cleaning section.

Errors, time dependent fields and other imperfections in a real machine can never be modeled accurately enough in a simulation so the dynamic aperture calculated by a simulation always exceeds the measured dynamic aperture. Observations at the Tevatron, HERA, and SPS have shown that the agreement between the measured and calculated dynamic aperture varies between $20-100 \%$. As a consequence the LHC, for example, requires that the calculated dynamic aperture after $10^{5}$ turns at both injection and collision (with only magnetic nonlinearities) equal $12 \sigma$. It is useful to consider the sources of discrepancies between the model and the accelerator, listed for example by Koutchouk [7] in Table 9. The LHC dynamic aperture calculations from [7] are provided in Table 10.

\begin{tabular}{|l||c|c|}
\hline Source or Uncertainty & Impact & D.A in $\sigma$ \\
\hline Target DA after $10^{5}$ turns & & 12.0 \\
Finite mesh size & $-5 \%$ & \\
Linear imperfections & $-5 \%$ & \\
Amplitude ratio $x_{i} / y_{i}$ & $-5 \%$ & \\
Extrapolation to $4 \times 10^{7}$ turns & $-7 \%$ & 9.6 \\
\hline Time-dependent multipoles & $-10 \%$ & \\
Ripple & $-10 \%$ & \\
\hline Safety margin & $-20 \%$ & \\
\hline Long-term DA & & 6.2 \\
\hline \hline
\end{tabular}

Table 9: Sources of uncertainties in the numerical calculation of dynamic aperture, taken from [7].

The linear imperfections include orbit errors due to misalignments and gradient errors and the loss of 5\% is assigned after correction of these errors. Since the errors listed above are just as likely for the VLHC, it seems reasonable to adopt a dynamic aperture requirement of $12 \sigma$ after $10^{5}$ turns.

The dynamic aperture in the LHC at injection is dominated by systematic multipoles in the dipoles. The lowest order allowed harmonics - sextupole and decapole - are corrected by small correction coils at the end of each LHC dipole.

\subsubsection{Scaling the Main Injector Errors}

The field harmonics in the low-field magnets will be measured in the summer or fall of 2001. As a starting hypothesis, the errors in the Main Injector are used as a scaling basis, assuming that the mechanical errors scale with the pole tip gap. Thus we assume that the field errors in the VLHC low field transmission line magnets at a pole tip radius of $10 \mathrm{~mm}$ are the same as the errors in the Main Injector magnets at a pole tip radius of $1 \mathrm{inch}$.

The expansion for the field error in a dipole magnet can be written in terms of coefficients $\left(b_{n}, a_{n}\right)$ as

$$
\Delta\left[B_{y}+i B_{x}\right](r, \theta)=B_{0} \sum_{n=1}^{\infty}\left(b_{n}+i a_{n}\right)\left(\frac{r}{R_{r e f}}\right)^{n} e^{i n \theta}
$$

\begin{tabular}{|l||c|c|}
\hline Non-linearity & Injection & Collision \\
\hline Chromaticity sextupoles & 28 & $\approx 70$ \\
Multipoles in dipoles & 6.5 & $>27$ \\
Multipoles in lattice quads & $\approx 12$ & $>27$ \\
Multipoles in low- $\beta$ quads & $>23$ & 6.5 \\
Long-range beam-beam kicks & 6.5 & 6 \\
\hline
\end{tabular}

Table 10: The dynamic aperture in units of rms beam size $\sigma$ due to various nonlinearities in the LHC, taken from [7]. 
at a radius $r$ and azimuth $\theta$. Here $B_{0}$ is the main dipole field and $R_{r e f}$ is the reference radius. Our assumption is that the relative errors are the same at each azimuth $\theta$,

$$
\frac{\Delta\left[B_{y}+i B_{x}\right]^{M I}(r=25.4 \mathrm{~mm}, \theta)}{B_{0}^{M I}}=\frac{\Delta\left[B_{y}+i B_{x}\right]^{V L H C}(r=10 \mathrm{~mm}, \theta)}{B_{0}^{V L H C}}
$$

The bend fields in the Main Injector at $8 \mathrm{GeV}$ and in the low field VLHC at an injection energy of $1 \mathrm{TeV}$ are nearly the same: $B_{0}^{M I}=0.101 \mathrm{~T}, B_{0}^{V L H C}=0.098 \mathrm{~T}$.

We make the somewhat stronger assumption that the equality is satisfied for every harmonic $n$ so that we obtain the following scaling relation between the multipole coefficients in the Main Injector and those in the low field VLHC,

$$
\left[\frac{b_{n}}{R_{r e f}^{n}}, \frac{a_{n}}{R_{r e f}^{n}}\right]^{V L H C}=(2.54)^{n} \times\left[\frac{b_{n}}{R_{r e f}^{n}}, \frac{a_{n}}{R_{r e f}^{n}}\right]^{M I}
$$

\begin{tabular}{|c||c|c||c|c|}
\hline Order $n$ & $\left\langle b_{n}\right\rangle$ & $\left\langle a_{n}\right\rangle$ & $\sigma\left(b_{n}\right)$ & $\sigma\left(a_{n}\right)$ \\
\hline 2 & -0.600 & 0.000 & 0.600 & 0.200 \\
3 & 0.000 & 0.00 & 0.149 & 0.300 \\
4 & 0.300 & 0.000 & 0.300 & 0.150 \\
5 & 0.000 & 0.000 & 0.100 & 0.500 \\
6 & 0.000 & 0.000 & 0.250 & 0.250 \\
\hline
\end{tabular}

Table 11: Systematic \langle\rangle and random $\sigma$ moments of multipole coefficients used for tracking the low field ring, scaled from Main Injector magnets using Equation (8). The coefficients are shown in units of $10^{-4}$, at a reference radius of $10 \mathrm{~mm}$.

Table 11 shows the derived harmonics for the low field magnets. We have assumed that the measured systematic skew harmonics in the Main Injector magnets are the result of the top down asymmetry in the magnet bus work [8]. This should not be an issue for the low-field magnets since the bus work is symmetric, and so the nonlinear skew harmonics have been set to zero.

\subsubsection{Dynamic Aperture at Injection}

The field errors in the arc magnets will determine the dynamic aperture at injection energy. The field quality in the transmission line magnets is not know as of this writing. We assume that a good approximation to these errors may be obtained by scaling the errors of the Main Injector magnets. The dynamic aperture is calculated by tracking single particles, usually for 1024 turns, through magnets with these errors. The main issue is to determine whether the field quality is good enough to meet the target set for the acceptable dynamic aperture. If not, a nonlinear corrector system that increases the dynamic aperture to the target value must be developed.

Our aim here is to make a rough estimate of the dynamic aperture with a limited number of initial conditions and with a small subset of machine imperfections. These calculations can be refined at a later stage when more information on the field quality is available. For most calculations reported here, particles are tracked for 1024 turns without synchrotron oscillations in a perfectly aligned lattice. The only nonlinearities are the chromaticity sextupoles and the field errors in the arc magnets. Sources of coupling are not introduced so correction with skew quadrupoles is not necessary. The fractional tunes are set to the Tevatron values $\nu_{x}=0.581, \nu_{y}=0.575$.

Figure 15 shows the dynamic aperture with only chromaticity sextupoles, only random errors, only systematic errors and all the errors. The dynamic aperture with all errors is nearly the same as that with only the random errors. Figures 16 and 17 show the dynamic apertures with only random and with only systematic errors.

Tracking up to 1024 turns with all the field errors at injection shows that the dynamic aperture is about $20 \sigma$ as shown in Table 12. Increasing the number of turns to 100,000 typically results in a decrease of the dynamic aperture by about $2-3 \sigma$. This would indicate that the target dynamic aperture of $12 \sigma$ at 100,000 turns should be met with this set of assumed errors.

\section{Improving the dynamic aperture}

The impact of linear coupling is reduced when there is a difference in the integer part of the horizontal and vertical tunes. In the perfect aligned lattice there are no sources of linear coupling. Nevertheless we studied the influence of tune splits on the 


\section{Dynamic Aperture of VLHC Low Field Lattice}

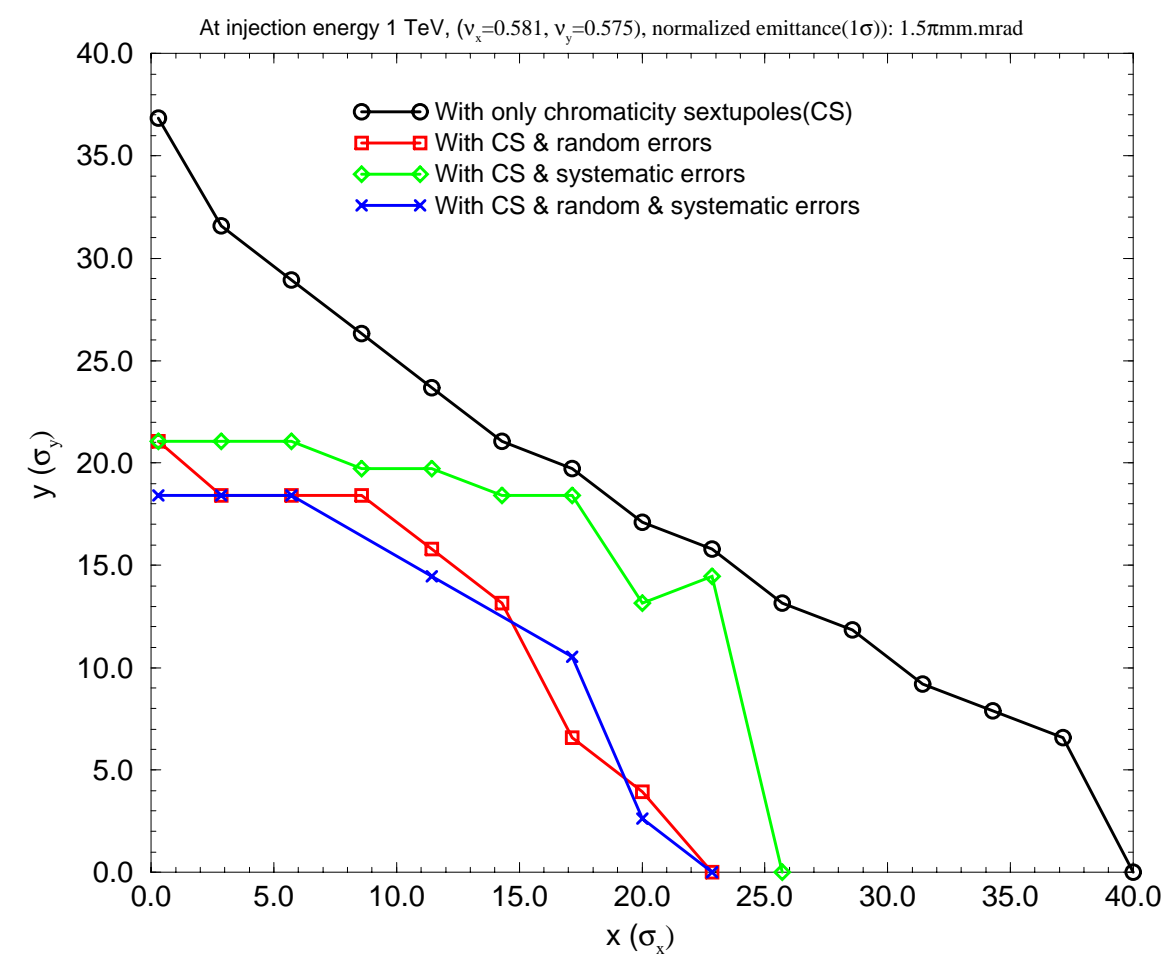

Figure 15: Dynamic aperture at injection with only chromaticity sextupoles, only random errors, only systematic, and all errors.

\begin{tabular}{|l|c|c|}
\hline Errors & \multicolumn{2}{|c|}{ Dynamic Aperture $(\sigma)$} \\
& Average & Minimum \\
\hline Only chromaticity sextupoles $(\mathrm{CS})$ & 30.9 & 25.4 \\
CS and only random errors & 20.0 & 18.4 \\
CS and only systematic errors & 23.4 & 21.1 \\
CS and all errors & 19.7 & 18.4 \\
\hline CS and only random errors and $\sigma\left(b_{2}\right)=\sigma\left(a_{2}\right)=0$ & 20.4 & 18.6 \\
CS and only random errors and $\sigma\left(b_{3}\right)=\sigma\left(a_{3}\right)=0$ & 20.2 & 17.6 \\
CS and only $\left\langle b_{2}\right\rangle \neq 0$ & 31.5 & 25.2 \\
CS and only $\left\langle b_{4}\right\rangle \neq 0$ & 24.5 & 21.1 \\
\hline
\end{tabular}

Table 12: Dynamic aperture at injection energy with various sets of errors in the arc magnets. These results show that the random errors together have a somewhat larger impact on the dynamic aperture than the two "allowed" systematic components, $\left\langle b_{2}\right\rangle$ and $\left\langle b_{4}\right\rangle$. The dynamic aperture with all the errors is about the same $(20 \sigma)$ as without the systematic errors. 
Dynamic Aperture of VLHC Low Field Lattice

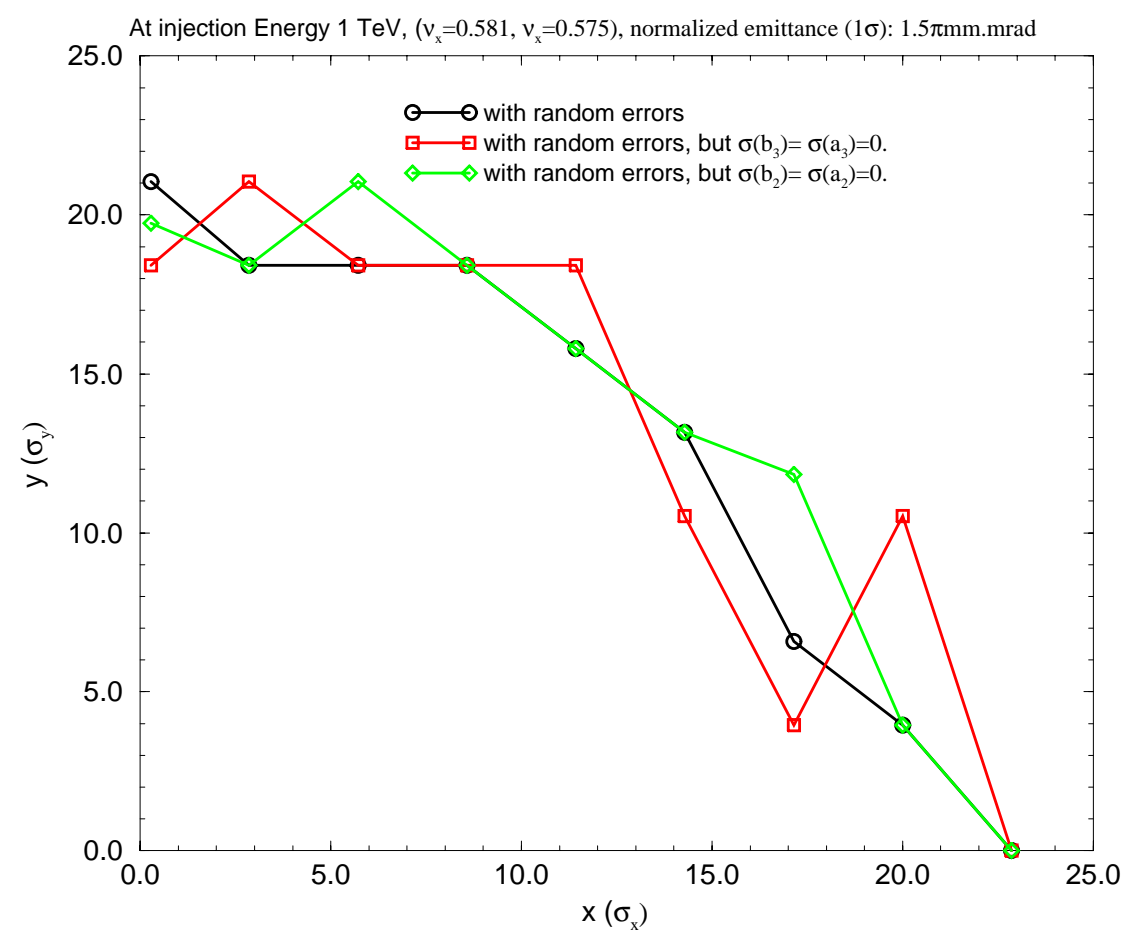

Figure 16: Dynamic aperture at injection with only random errors.

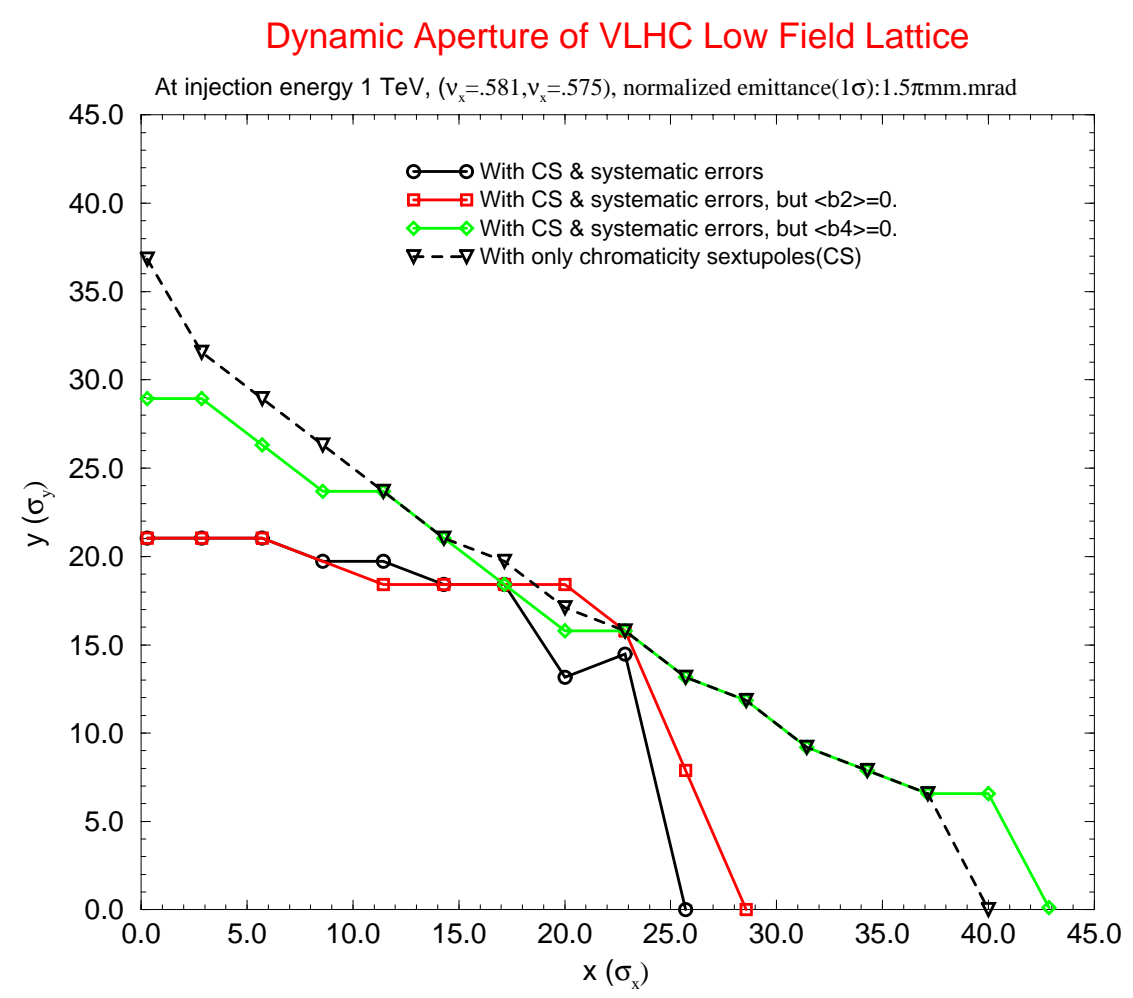

Figure 17: Dynamic aperture at injection with only systematic errors. 


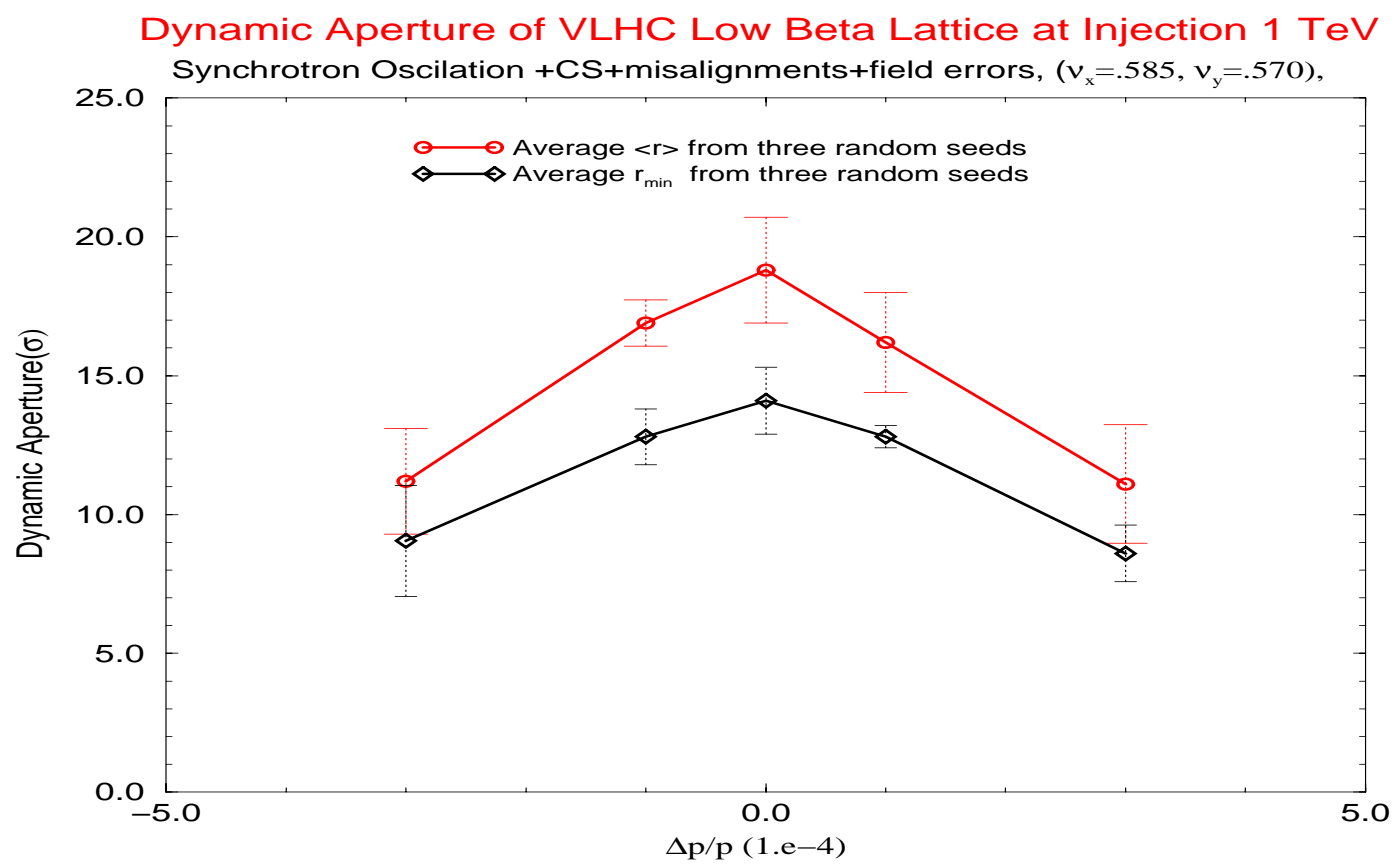

Figure 18: Dynamic aperture with synchrotron oscillations and closed orbit errors. Particles were tracked for 10,000 turns and with three random seeds.

dynamic aperture for the cases $\nu_{x}-\nu_{y}= \pm 2, \pm 4, \pm 6$. In no case did the dynamic aperture improve and in some cases the aperture was reduced significantly, presumably due to the beta beating.

If at a future stage the revised field harmonics show that the systematic components have a significant impact on the dynamic aperture, then one way of mitigating their effects is to alternate the signs of the systematic components after every cell. The polarities of the field components can be switched by reversing the lead and return ends of the magnet. This requires not only rotating the magnets end to end but also powering them "backwards". In this scenario, the even normal components $\left\langle b_{2 n}\right\rangle$ and the odd skew components $\left\langle a_{2 n+1}\right\rangle$ change sign [9]. If the systematic components have opposite signs between adjacent cells, there will a partial cancellation in the signs of the nonlinear kicks and the amplitude growth will not be as strong. The dynamic aperture with a configuration where the signs of all the systematic components $\left\langle b_{n}\right\rangle$ reverse every successive cell was studied and was found to increase the dynamic aperture significantly.

After correction of the orbit with misaligned magnets, the rms closed orbit deviations were reduced to $\langle x\rangle_{\mathrm{rms}}=0.22$ $\mathrm{mm},\langle y\rangle_{r m s}=0.21 \mathrm{~mm}$. The fractional tunes were $\nu_{x}=0.585, \nu_{y}=0.573$. Figure 18 shows the average and minimum dynamic aperture with synchrotron oscillations and closed orbit errors as a function of the momentum deviation amplitude. The average and minimum are taken over three random seeds. The average dynamic aperture at $\delta p / p=0$ is $19 \sigma$ while at $\delta p / p=3 \times 10^{-4} \approx 3 \sigma_{p}$, the average dynamic aperture drops to about $11 \sigma$ which is only slightly under the target value.

\subsubsection{Dynamic Aperture at Collision}

The dynamic aperture at collision optics will be dominated by the field errors of the IR quadrupoles due to the large beam sizes in these magnets. This is true for the LHC design and was also true for the SSC design. The IR quadrupoles with their high gradients will be built with "conventional" superconducting magnets. The nonlinear harmonics in these magnets are much better understood following decades of development and also through the Fermilab program of building these quadrupoles for the LHC. The errors in the arc magnets built with conventional technology usually are not significant in determining the dynamic aperture at top energy. For example, the target dynamic aperture in the LHC at top energy is set at $12 \sigma$ with only the IR quadrupole field errors. We will adopt the same target dynamic aperture of $12 \sigma$ in the simulations.

The arc magnets in the Stage 1 collider, however, are built with transmission line magnets which have different characteristics from conventional superconducting magnets. At high current, the field in the iron saturates leading to a drop in the gradient and a large negative sextupole component $\left(b_{2}\right)$. While the change in gradient will be compensated by quadrupole correctors in 


\begin{tabular}{|c|c|c|c|c||c|c|}
\hline Order $n$ & $\left\langle b_{n}\right\rangle$ & $\left\langle a_{n}\right\rangle$ & $d\left\langle b_{n}\right\rangle$ & $d\left\langle a_{n}\right\rangle$ & $\sigma\left(b_{n}\right)$ & $\sigma\left(a_{n}\right)$ \\
\hline 2 & 0 & 0 & 0.5 & 0.5 & 0.9 & 0.9 \\
3 & 0 & 0 & 0.1 & 0.1 & 0.3 & 0.3 \\
4 & 0 & 0 & 0.05 & 0.05 & 0.06 & 0.06 \\
5 & 0.015 & 0 & 0.1 & 0.01 & 0.1 & 0.05 \\
6 & 0 & 0 & 0.005 & 0.005 & 0.005 & 0.005 \\
7 & 0 & 0 & 0.002 & 0.002 & 0.003 & 0.003 \\
8 & 0 & 0 & 0.0005 & 0.0005 & 0.001 & 0.001 \\
9 & 0.0002 & 0.0 & 0.001 & 0.0003 & 0.0005 & 0.0005 \\
\hline
\end{tabular}

Table 13: Field harmonics of the low field IR quadrupoles [10]. $\left\langle b_{n}\right\rangle,\left\langle a_{n}\right\rangle$ are the systematic errors, $d\left\langle b_{n}\right\rangle, d\left\langle a_{n}\right\rangle$ are the uncertainties in the systematics, and $\sigma b_{n}, \sigma a_{n}$ are the random errors. The errors are expressed in units of $10^{-4}$, at a reference radius of $10 \mathrm{~mm}$.

every cell to maintain the correct phase advance per cell, the compensation of the sextupole component is a more serious issue. If the nonlinear fields due to magnet saturation have a significant impact on the dynamic aperture, then this could potentially limit the maximum energy reach of the low field collider.

The straightforward approach to compensating the saturation $b_{2}$ component is to use the chromaticity sextupoles in the cells to correct the linear chromaticity. At large $\left|b_{2}\right|$ this leads to strong chromaticity sextupoles lumped at two locations in the cell, one SF next to the focusing magnet (F), the other SD next to the defocusing magnet (D). A calculation of the dynamic aperture at $20 \mathrm{TeV}$ without the IR errors but with the $b_{2}$ component in the arc magnets due to saturation shows that the dynamic aperture is quite large even up to 10 units of $b_{2}$. This is seen in Figure 19. The integrated sextupole strengths required to compensate the saturation sextupole component are shown in Figure 20. At $b_{2}=0$, integrated strengths required for the $\mathrm{F}$ and $\mathrm{D}$ type sextupoles are $(946,1761) \mathrm{T} / \mathrm{m}$ respectively. At positive $b_{2} \leq 3$, the $\mathrm{F}$ sextupoles decrease and $\mathrm{D}$ sextupoles increase in magnitude and vice versa at negative $b_{2} \geq-3$. At larger absolute values of $b_{2}$, both sextupoles increase in magnitude. If required, additional sextupoles could be placed in the $1 \mathrm{~m}$ gap between the $\mathrm{F}$ and $\mathrm{D}$ and the $\mathrm{D}$ and $\mathrm{F}$ magnets to correct the chromaticity generated by the iron saturation. This would lower the integrated sextupole strengths and allow for larger values of $b_{2}$ to be compensated.

The impact of magnet saturation should, however, be determined in the presence of the dominant field errors of the IR quadrupoles. We have based our dynamic aperture calculations on the expected field errors calculated by G. Ambrosio et al. [10], shown in Table 13.

The dynamic aperture was calculated for three seeds for the random errors with the signs of the uncertainties in the systematics alternating between positive and negative. The random errors were truncated at $3 \sigma$. For each seed the dynamic aperture was calculated with $-10 \leq b_{2} \leq 10$. Figure 21 shows the dynamic aperture averaged over the three seeds for each value of $b_{2}$.

At $b_{2}=0$, the dynamic aperture drops to around $20 \sigma$ in the presence of the IR errors, compared to a value of around $130 \sigma$ without these errors but only chromaticity sextupoles. Figure 21 shows that the average dynamic aperture varies over a small range of 20-23 $\sigma$ when $\left|b_{2}\right| \leq 10$. Clearly the saturation sextupoles do not have a significant impact on the dynamic aperture at $20 \mathrm{TeV}$. The maximum tolerable value of $b_{2}$ may instead be limited by the sextupole strengths required to correct the chromaticity.

\subsection{Tolerances and Corrections}

\subsubsection{Arcs}

\section{Closed orbit}

Distortions of the ideal closed trajectory in the collider will be primarily generated by transverse alignment errors. The trajectory in the horizontal plane will also be influenced by errors in the main bend field of the magnets, and the vertical trajectory will be further distorted due to roll errors in the main bending field.

For a bending magnet of length $L$ with gradient $B^{\prime}$ which is displaced transversely from its ideal position by an amount $d$, a particle with magnetic rigidity $B \rho=p / e$ will have its trajectory deflected through an angle $\theta=\left(B^{\prime} L / B \rho\right) \cdot d$. For the low field collider, the gradient magnets have lengths roughly equal to the half-cell length and will be supported along its length by several adjustable magnet stands. If the arc magnets are divided into $n$ equal-length sections for support and alignment, then the rms expected closed orbit deviations in the horizontal $(x)$ and vertical $(y)$ degrees of freedom at maximum $\beta$ locations in 
Dynamic Aperture at $20 \mathrm{TeV}$ with saturation sextupoles

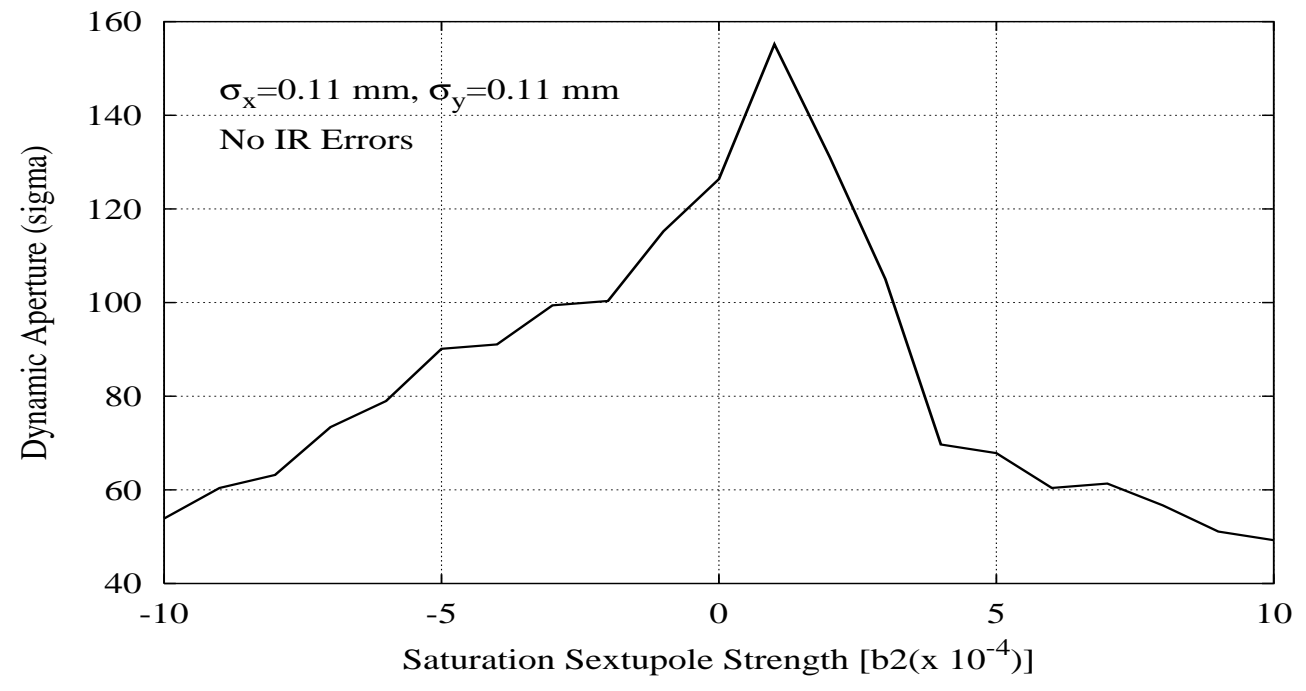

Figure 19: Dynamic aperture at top energy as a function of the saturation $b_{2}$ in the arc magnets without field errors in the IR quadrupoles.

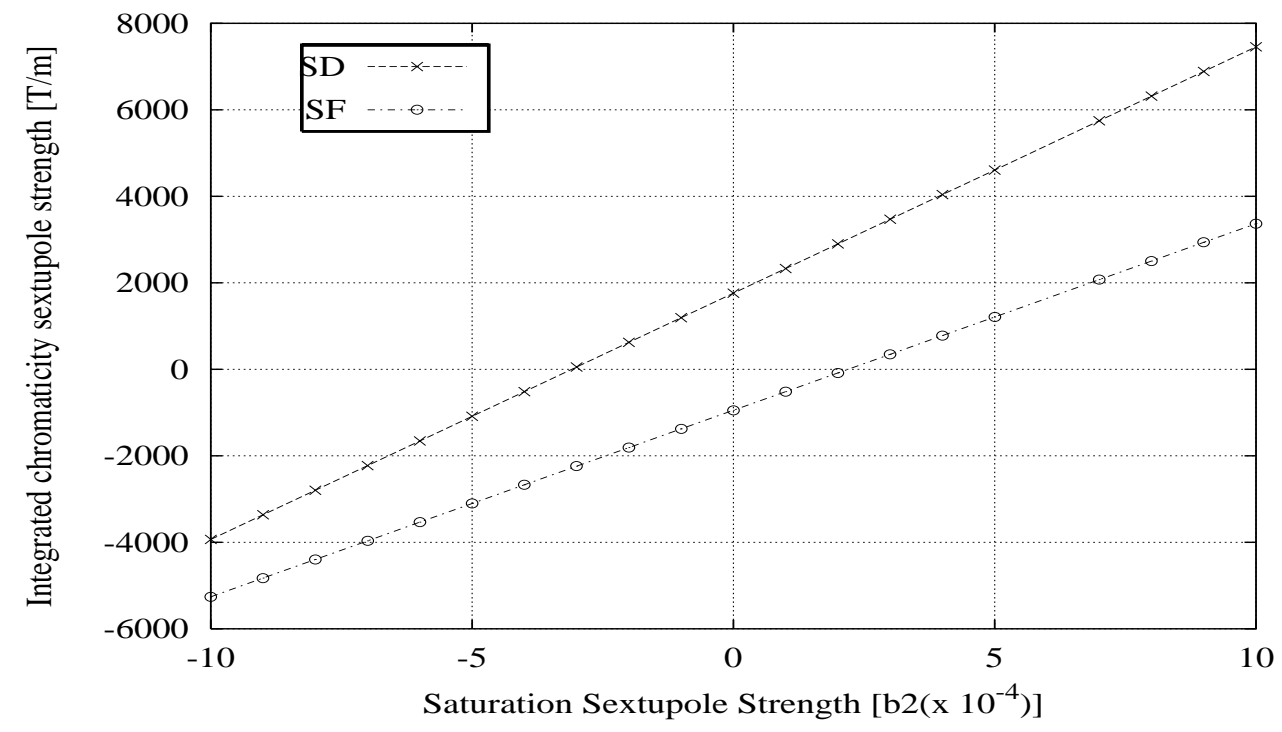

Figure 20: Strengths of the chromaticity correction sextupoles as a function of the saturation $b_{2}$. 
Dynamic Aperture with IR Errors and Saturation Sextupoles

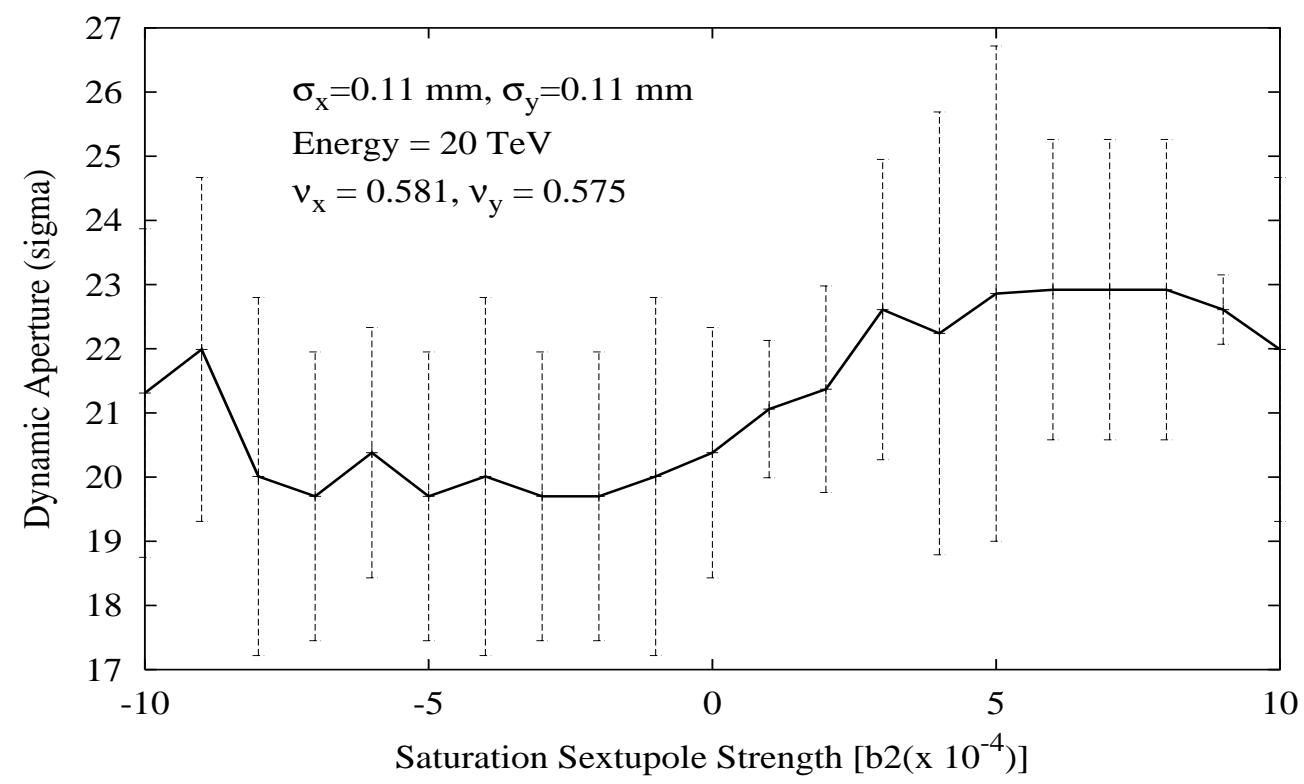

Figure 21: Dynamic aperture as a function of the saturation $b_{2}$ in the arc magnets with errors in the IR quadrupoles. The error bars represent the variation over three seeds for the random errors. The dynamic aperture does not change significantly over this range of $b_{2}$.

the arcs are given by

$$
\Delta \hat{x}_{c o}=\frac{\sqrt{\hat{\beta}\langle\beta\rangle}}{2|\sin \pi \nu|} \sqrt{\frac{N}{2}}\left(\frac{B_{0} L}{B \rho}\right) \sqrt{\frac{1}{n}\left(\frac{B^{\prime} d_{r m s}}{B_{0}}\right)^{2}+\left(\frac{\Delta B}{B_{0}}\right)_{r m s}^{2}}
$$

and

$$
\Delta \hat{y}_{c o}=\frac{\sqrt{\hat{\beta}\langle\beta\rangle}}{2|\sin \pi \nu|} \sqrt{\frac{N}{2}}\left(\frac{B_{0} L}{B \rho}\right) \sqrt{\frac{1}{n}\left[\left(\frac{B^{\prime} d_{r m s}}{B_{0}}\right)^{2}+\phi_{r m s}^{2}\right]}
$$

where $\left(\Delta B / B_{0}\right)_{r m s}$ is the rms field strength deviation of the full magnet, $d_{r m s}$ is the rms displacement at the $n$ locations, and $\phi_{r m s}$ is the rms roll angle (with respect to vertical) at the $n$ locations within a half-cell. $N$ is the number of half-cells in the collider.

For typical values of the above variables we use $n=5$ over a half cell length of $L=67 \mathrm{~m}, \hat{\beta}=411 \mathrm{~m},\langle\beta\rangle=250 \mathrm{~m}, B_{0}=$ $2 \mathrm{~T}, B^{\prime}=9 \mathrm{~T} / \mathrm{m}, d_{r m s}=250 \mu \mathrm{m}, \phi_{r m s}=0.5 \mathrm{mrad}$, and $\left(\Delta B / B_{0}\right)=10^{-4}$. From a distribution of errors over the 780 arc cells in the collider, we obtain expected rms orbit errors on the scale of

$$
\begin{aligned}
& \Delta \hat{x}_{c o} \approx 9.5 \mathrm{~mm}, \\
& \Delta \hat{y}_{c o} \approx 10.3 \mathrm{~mm} .
\end{aligned}
$$

The closed orbit will clearly venture outside any reasonable beam pipe radius.

To correct the orbit distortions, we assume dipole correctors are placed at maximum $\beta$ locations in the arcs, and a standard 3-bump algorithm is performed. The corrector strength necessary to perform the correction using the parameters introduced above would be

$$
\Theta_{x}=\frac{\sqrt{2}}{\sin \mu} \sqrt{\frac{\langle\beta\rangle}{\hat{\beta}}}\left(\frac{B_{0} L}{B \rho}\right) \sqrt{\frac{1}{n}\left(\frac{B^{\prime} d_{r m s}}{B_{0}}\right)^{2}+\left(\frac{\Delta B}{B_{0}}\right)_{r m s}^{2}}
$$


and

$$
\Theta_{y}=\frac{\sqrt{2}}{\sin \mu} \sqrt{\frac{\langle\beta\rangle}{\hat{\beta}}}\left(\frac{B_{0} L}{B \rho}\right) \sqrt{\frac{1}{n}\left[\left(\frac{B^{\prime} d_{r m s}}{B_{0}}\right)^{2}+\phi_{r m s}^{2}\right]}
$$

where $\mu$ is the cell phase advance. For $\mu=90^{\circ}$, and the same parameter values as before, the rms corrector strengths are

$$
\begin{aligned}
& \Theta_{x} \approx 2.3 \mu \mathrm{rad}, \\
& \Theta_{y} \approx 3.2 \mu \mathrm{rad} .
\end{aligned}
$$

At $20 \mathrm{TeV}$, a $3 \mu \mathrm{rad}$ bend angle requires an integrated field strength of $0.2 \mathrm{~T}-\mathrm{m}$. To be able to correct at the 2.5- $\sigma$ level, the steering correctors should have strengths of approximately $0.5 \mathrm{~T}-\mathrm{m}$.

\section{Quadrupole saturation}

The quadrupole gradient in the transmission line magnets drops steeply at high fields due to saturation of the iron. Quadrupole correctors, one in each plane, will be placed in every arc cell to maintain the required phase advance of $90^{\circ}$ per cell. At present these correctors are $0.5 \mathrm{~m}$ long and placed at the beginning of each half cell. A 5\% drop in the gradient of the main magnets at $2.0 \mathrm{TeV}$ will require a gradient of $80 \mathrm{~T} / \mathrm{m}$ in these correctors to maintain the phase advance in the cells.

\section{Tune control}

Sources of errors in the tune include gradient errors and closed orbit distortions in the nonlinear magnets. Quadrupole correctors in the arcs will be used to control the global tunes and to split the integer tunes to reduce the sensitivity to systematic linear coupling sources.

\section{Beta beating}

It will be important to restrict the beta-beating to a low value everywhere, both for on-momentum and off-momentum particles. It will be specially important to isolate the interaction regions from beta-beating errors generated elsewhere in the ring. Quadrupoles at the entrances and exits of the arcs and at the entrances and exits of the straight sections can be used to control both the amplitude and phase of $\Delta \beta / \beta$.

\section{Coupling}

Sources of coupling include skew quadrupole errors in the main magnets, rolls of the gradient magnets and vertical orbit distortion through the nonlinear magnets. The tolerance for the allowed coupling may be set by requiring that the minimum tune split be less than 0.05 before correction. Larger values make coupling correction with skew quadrupoles difficult.

Skew quadrupoles placed in regions of horizontal dispersion will generate vertical dispersion which will need additional correction. It is therefore preferable to place these quadrupoles in straight sections. Two families of skew quadrupoles in the utility straight sections and the IRs will be sufficient to control the effects due to the difference resonance $\nu_{x}-\nu_{y}=p$. These families will be placed so that the difference in phases $\psi_{x}-\psi_{y}$ differs by $\pi / 2$ at the families. The coupling sum resonance $\nu_{x}+\nu_{y}=p$ may also need to be corrected (depending on the strength of the skew quadrupole errors). In this case an additional two families will be required, placed so that $\psi_{x}+\psi_{y}$ differs by $\pi / 2$ at these families.

\section{Dispersion control}

Normal quadrupole gradient errors and skew quadrupole gradient errors will contribute to horizontal and vertical dispersion beating respectively. The errors generated in the arcs and utility straight sections will be corrected either by quadrupole correctors or by the closed orbit correctors. It will be important to keep the dispersion at the IPs very small. Local dispersion correction within the IRs will be necessary to correct the dispersion generated by the crossing angle. 


\section{Chromaticity and momentum dependent beta functions}

The linear chromaticity is controlled by two families of sextupoles, one in each plane, placed in every arc cell. This will suffice provided the nonlinear change of tune with momentum is small over the required momentum aperture. If the global nonlinear chromaticity requires correction, then two families of sextupoles in each plane for the $90^{\circ}$ cells will be required. It would therefore be desirable to place sextupoles (of say the F type) in every other cell on one bus and the other family of sextupoles in the other cells on another bus. Similarly for the other type.

In addition to the chromaticity due to the quadrupoles, there is additional chromaticity due to sextupole fields caused by iron saturation at fields close to $2 \mathrm{~T}$. This chromaticity must also be compensated by the chromaticity sextupoles. This has been discussed in the section on the dynamic aperture at collision.

If the beta function is strongly dependent on the momentum, then it can lead to an increased spot size if not corrected at the IPs and to emittance growth due to injection errors if not corrected at the injection point. Correcting the momentum dependence of the beta functions will require additional sextupole families.

\subsubsection{Interaction Regions}

Alignment tolerances in the IRs

These tolerances are more stringent for the IR quadrupoles than for any other magnets in the ring. Several criteria have to be met including:

- Dispersion at the IP should be small, both to attain maximum luminosity and to avoid synchro-betatron resonances.

- Beam excursions in the triplets should be limited to preserve the dynamic aperture.

- Separations between the beams at the parasitic collision points should not decrease.

Table 14 shows the effects of the IR quadrupole misalignments on the beam optics. In the IR quadrupoles the beta functions change rapidly and are not symmetric about the quadrupole center. As a consequence, pitch and yaw misalignments will introduce substantial beam separations at the IPs if not corrected. Due to the crossing angle a roll misalignment of an IR quadrupole will introduce orbit shifts in the plane orthogonal to the crossing plane. This is in addition to the extra coupling generated by the roll angle. Thus misalignments in all six degrees of freedom of these IR quadrupoles need to be well controlled and corrected. Other deformations in straightness, such as the natural sag of the magnets, must be corrected with the support structures of these magnets.

\begin{tabular}{|ll|}
\hline MISALIGNMENT & EFFECT \\
Transverse displacements & $\begin{array}{l}\text { Orbit shift } \\
\text { Tune shift (with nonlinear magnets) }\end{array}$ \\
Longitudinal displacement & Tune shift, beta beat, dispersion beat \\
Pitch and Yaw & $\begin{array}{l}\text { Orbit shift } \\
\text { Tune shift (with nonlinear magnets) } \\
\text { Roll }\end{array}$ \\
& $\begin{array}{l}\text { Enhanced coupling, orbit shift(with crossing angle) } \\
\text { Beta beat, dispersion beat }\end{array}$ \\
\hline
\end{tabular}

Table 14: Rigid misalignments of quadrupoles and their effects on the beam optics.

\section{Offsets at the IPs}

The orbit offset at the IP $z_{\text {of } f \text { set }}(I P)$ due to a transverse displacement $\Delta z_{Q}$ of an IR quadrupole (assumed to be at 90 degrees from the IP) is

$$
\frac{z_{o f f s e t}(I P)}{\Delta z_{Q}} \propto \frac{\sqrt{\beta^{*} \beta_{Q}}}{F}
$$


Here $\beta_{Q}$ is the beta function at the quadrupole and $F$ is the focal length of the displaced quadrupole. The maximum offset of the quadrupole that can be corrected with orbit correctors is proportional to this ratio $z_{\text {of fset }}(I P) / \Delta z_{Q}$. In Table 15 we compare the parameters for the quadrupole where the beta function is the largest. Tolerances for the LHC quadrupoles were

\begin{tabular}{|l|c|c|}
\hline Parameter & LHC IR & VLHC (LF) IR \\
\hline$\beta^{*}[\mathrm{~m}]$ & 0.5 & 0.3 \\
$\beta^{\text {max }}[\mathrm{m}]$ & 4567 & 11263 \\
Focal length $F[\mathrm{~m}]$ & 21.2 & 18.7 \\
$z_{\text {offset }}(I P) / \Delta z_{Q}$ & $\sim 2.3$ & $\sim 3.1$ \\
\hline
\end{tabular}

Table 15: Parameters of the strongest IR quadrupole in the LHC and in the low field VLHC

derived in [11]. The rms tolerance for the offset was obtained to be $0.27 \mathrm{~mm}$. Scaling this number by the ratio 2.3/3.1, we obtain a tolerance of $0.2 \mathrm{~mm}$ for the IR quadrupoles. This assumes that corrector strengths similar to those in the LHC (in kick angle) will be used in the low field VLHC.

Correctors to correct the orbit at the IP are best placed at 90 degrees from the IP. As in the LHC, correction packages with dipole and skew dipole correction coils should be placed at several high beta locations next to the IR quadrupoles. Spaces between Q2 and Q3 and after Q3 would be ideal locations for these correctors.

\section{Quadrupole roll angles}

Tolerances on the allowed roll angles of the IR quadrupoles may be set by specifying the minimum tune split tolerable before correction. We will assume that this tune split due to roll angles in all sixteen quadrupoles from the two IRs is less than 0.05 before correction. The minimum tune difference due to a roll angle $\Delta \phi$ is given by

$$
\Delta Q_{\text {min }}=\frac{\sqrt{\beta_{x} \beta_{y}}}{\pi} K L_{q} \Delta \phi
$$

An rms roll angle of $0.5 \mathrm{mrad}$ can be tolerated for the IR quads, assuming that all the IR quadrupole assemblies include a skew quadrupole corrector.

Normally two families of skew quadrupoles are required to correct both the real and imaginary parts of the coupling resonance term. However the phase advance through the IRs is very small and so the imaginary part of the resonance term is negligibly small. Thus only a single skew quadrupole on each side of the IP will suffice to locally correct the coupling in the IR. The integrated strength of the skew quadrupole corrector may be calculated from [11]

$$
\begin{aligned}
(K l)_{\text {skew }} & =-\frac{1}{\left(\sqrt{\beta_{x} \beta_{y}}\right)_{s} \cos \theta_{s}} \mathcal{R} \\
\mathcal{R} & =2 \sum_{i}^{8}\left(\sqrt{\beta_{x} \beta_{y}}\right)_{i} K_{i} \Delta \phi_{i} l_{i} \cos \theta_{i}
\end{aligned}
$$

The phase argument at a quadrupole distant $s$ from the IP is $\theta_{i}=\left[\psi_{x}-\psi_{y}-\left(\nu_{x}-\nu_{y}-p\right) \frac{s}{R}\right]_{i}$. $\mathcal{R}$ is the real part of the difference coupling resonance term driven by the rolled quadrupoles in the IR, each with a roll $\Delta \phi_{i}$ where $i$ runs over all the eight quadrupoles in each IR. These expressions can be used to calculate the rms roll angle that can be corrected with the available skew quadrupole correctors.

Table 16 summarizes the misalignment tolerances for the IR quadrupoles. These are based on scaling from tolerances derived for the LHC and should be replaced at a later stage with more exact calculations.

\begin{tabular}{|l|c|}
\hline Misalignment & Tolerance \\
\hline Offsets [mm] & 0.2 \\
Longitudinal placement [mm] & 1 \\
Roll [mrad] & 0.5 \\
\hline
\end{tabular}

Table 16: Misalignment tolerances for the low field VLHC IR quadrupoles derived by scaling from the LHC IR quadrupole tolerances [11]. 


\section{Crossing angle}

A crossing angle is required to separate the beams at the parasitic collisions. As is well known, the crossing angle has several effects on the linear and nonlinear optics and beam dynamics. Some of these effects which require correction include:

- Dispersion wave due to orbit offset in the quadrupoles

- Change in coupling due to skew multipole errors in the quadrupoles

- Feed down of higher order multipoles.

Local dispersion control is required to prevent the dispersion waves from propagating into the arcs. This can be performed adjusting pairs of quadrupoles in the cells adjacent to the IRs where there is natural dispersion. A scheme such as this was proposed for the SSC [12]. Correction of local coupling from this and other sources will require skew quadrupoles in the IR as already mentioned. The linear effects from feed-down of higher order multipoles will be corrected by orbit and tune correctors. Nonlinear effects will require nonlinear correction packages in the IRs similar to those in the LHC and RHIC.

\section{Local chromaticity correction}

A local chromaticity correction system for the IRs was proposed for the SSC [13] which increased the momentum aperture from about $2.5 \sigma_{p}$ with linear chromaticity correction to about $8 \sigma_{p}$ with correction of the nonlinear chromaticity generated by the IRs. A similar such system may be required for the IRs if the momentum aperture needs to be increased.

\subsection{Ground Motion and Emittance Growth}

Slow ground motion in the 300-ft deep dolomite Conco mine in Aurora, IL, about 3 miles west of Fermilab, is being measured. The goal of the studies is to record and analyze vertical ground motion in 8 points separated by 30 meters over a time interval of about 1 year. The experiment in Aurora mine started in October 2000. The first data reveal that dolomite mine motion is rather small and can be described by the ATL law with coefficient of $A=(6.8 \pm 2.7) \times 10^{-7} \mathrm{mum}^{2} / \mathrm{m} / \mathrm{s}[14,15]$. The resulting maximum orbit distortion that will be caused is of the order of $9 \mathrm{~mm} / \sqrt{\text { year }}$.

The only drawback of the Aurora mine experiment is the minimal number of sensors that limit statistics for studies of spatial correlations. Therefore, a system with about 20 sensors is needed, and long term measurements should be repeated in the larger scale. Analytical/computer studies of the slow ground motion effects and orbit correction system and procedures must be performed.

Turn-to-turn dipole magnetic field fluctuations and vibration of quadrupole magnets are of concern, because they can excite coherent beam motion. If the motion is not corrected over the decoherence time of about $1 / \xi$ beam-beam $\approx 1000$ turns, then the coherent motion will be converted into transverse emittance increase and can cause substantial emittance growth over about 10 hours of the store. So far, experimentally measured high-frequency ground or magnet vibrations do not pose a big problem for VLHC [16]. Nevertheless, more detailed analysis on how the lattice may affect the tolerances is needed. For example reference [17] claims that tolerances on vibration amplitude in a lattice with combined function magnets is about 3 times the tolerances in a separated function FODO lattices.

In 1999 experimental studies of high-frequency magnetic field fluctuations in the Tevatron superconducting dipole magnet were carried out. It was found that the effective rms $d B / B \approx 10^{-10}$ is about 10 times the tolerance for the VLHC, i.e. that which might cause emittance doubling after about 6 minutes. The measurements have to be repeated in the VLHC dipole prototypes. Again, we need to analyze and perform computer simulations for lattices with separated and combined function magnets.

Suppression of the emittance growth can be provided by low noise feedback. The first analysis of the required feedback system is presented in [18]. The system has to have sub-micron equivalent input noise. Design and test of such a system at the Tevatron is a desirable VLHC beam physics R\&D topic. 


\subsection{Stability Issues}

\subsubsection{The Rings, Injection, and RF}

In Stage 1, bunches are injected from the Tevatron into the low-field rings at $900 \mathrm{GeV}$ and are accelerated to $20 \mathrm{TeV}$. The rings of both phases are chosen to be of the same size, $2 \pi R=233.037 \mathrm{~km}$ in circumference, and same rf frequency. In order to have shorter bunches, the rf frequencies in both stages are chosen 9 times higher than that of the Tevatron, so that the bunches in the VLHC will be separated by $9 \mathrm{rf}$ buckets. The $\mathrm{rf}$ harmonic is $h=9 h_{T} R / R_{T}=371520$, where $h_{T}=1113$ is the rf harmonic of the Tevatron and $R_{T}=1.0 \mathrm{~km}$ is its mean radius.

Here, we concentrate on the stability issues of the low-field rings only, where the rf voltage has been chosen to be $V_{\text {rf }}=$ $50 \mathrm{MV}$. In order to avoid or reduce the possibility of longitudinal and transverse single bunch instabilities, the rms bunch area of bunches inside the low field buckets are chosen as $A=2.0 \mathrm{eV}$-s, very much larger than the $A_{T}=0.4 \mathrm{eV}$-s rms bunch area [19] of the Tevatron bunches at $900 \mathrm{GeV}$. However, with $V_{\mathrm{rf}}=50 \mathrm{MV}$, the maximum energy spread and bucket area are, respectively,

$$
\begin{gathered}
{\left[\frac{\Delta E}{E}\right]_{\text {bucket }}=\sqrt{\frac{2 e V_{\mathrm{rf}}}{\pi h|\eta| E}}=2.0910^{-3},} \\
A_{\text {bucket }}=\frac{8}{\omega_{0}} \sqrt{\frac{2 e V_{\mathrm{rf}} E}{\pi h|\eta|}}=5.01 \mathrm{eV} \text {-s }
\end{gathered}
$$

where $\eta=2.9110^{-5}$ is the slip factor of the VLHC rings in both stages, $f_{0}=\omega_{0} /(2 \pi)=1.29 \mathrm{kHz}$ their revolution frequency, and $E=900 \mathrm{GeV}$ the injection energy. This bucket is definitely too small for the rms $2 \mathrm{eV}$-s bunch at this energy. In the discussions below, we assume that the bunches in the low-field rings injected from the Tevatron will be of rms bunch area $0.4 \mathrm{eV}$-s. As we shall see, a bunch coalescing scheme will be implemented before storage, which will increase the rms bunch area of the final bunches to near the desired value.

Incidentally, the Tevatron of has its maximum voltage at $1 \mathrm{MV}$ only, the bunch length being too long and the momentum spread too small to fit the buckets at injection. Thus, the Tevatron bunches must be shortened by either a bunch rotation or the installation of a higher $\mathrm{rf}$ voltage before the transfer to the VLHC is possible. Some relevant $\mathrm{rf}$ and beam parameters of the Stage 1 ring are listed in Table 17 for convenience.

A bunch excited by forces in the vacuum chamber oscillates in the longitudinal phase space in modes describable by the radial mode parameter $n_{r}$ designating $n_{r}$ radial nodes and the azimuthal mode parameter $m$ designating $|m|$ azimuthal nodes. For a given $m$, the most easily excited radial mode is $n_{r}=|m|$ and only these radial modes will be included in the discussion below. Almost distribution independent, the spectrum for the mode designated by $m \neq 0$ peaks at frequency

$$
f_{(m)} \approx \frac{|m|+1}{2 \tau_{L}},
$$

where $\tau_{L}$ is the total length of the bunch in time. In Gaussian distribution, we approximate it by the $95 \%$ length $\tau_{L}=2 \sqrt{6} \sigma_{\tau}$, where $\sigma_{\tau}$ is the rms bunch length. Actually, the spectrum of this mode is nonzero only at the $m$-th synchrotron sidebands of, respectively, the revolution harmonics for longitudinal discussion and the betatron tune lines for transverse discussion. For $m=0$, the spectrum has a frequency spread from $-f_{(0)}$ to $+f_{(0)}$ with $f_{(0)}$ given by Eqn. (15). Both $f_{(0)}$ and $f_{(1)}$ are listed in Table 17. Note that these frequencies for the VLHC bunch modes are in the GHz range.

\subsubsection{Resistive Wall}

The longitudinal impedance of the wall of a cylindrical beam pipe of radius $b$ is, at angular frequency $\omega$ or harmonic $n$,

$$
\left[\frac{Z_{\|}}{n}\right]_{\text {wall,cyl }}=[\operatorname{sgn}(\omega)+j] \frac{R \rho}{b \delta_{1}}|n|^{-1 / 2},
$$

where $\rho$ is the resistivity of the beam pipe wall and

$$
\delta_{1}=\sqrt{\frac{2 \rho}{\omega_{0} \mu}}
$$

is the skin depth at the revolution harmonic and $\mu$ the magnetic permeability. The VLHC beam pipes for the low-field rings are warm with an elliptical cross section of radii $h=9 \mathrm{~mm}$ and $w=14.0 \mathrm{~mm}$. The longitudinal impedance is given by

$$
\left[\frac{Z_{\|}}{n}\right]_{\text {wall }}=\left[\frac{Z_{\|}}{n}\right]_{\text {wall,cyl }} F_{L}
$$


Table 17: Parameters of the Stage 1 ring at injection and storage. For the last row, see Sec. 2.5.5.

\begin{tabular}{|c|c|c|c|}
\hline & \multicolumn{2}{|c|}{ STAGE 1} & \\
\hline & Injection & Storage & \\
\hline \multicolumn{4}{|l|}{ Rings and RF } \\
\hline Circumference $C=2 \pi R$ & 233.037 & 233.037 & $\mathrm{~km}$ \\
\hline Energy $E$ & 0.9 & 20.0 & $\mathrm{TeV}$ \\
\hline Bunch separation & 9 & 9 & buckets \\
\hline Rf harmonic $h$ & 371520 & 371520 & \\
\hline Rf voltage $V_{\text {rf }}$ & 50 & 50 & \\
\hline Slip factor & $2.19010^{-5}$ & $2.19010^{-5}$ & \\
\hline Synchrotron tune $\nu_{s}$ & $8.4610^{-3}$ & $1.7910^{-3}$ & \\
\hline Max. energy spread & $2.0910^{-3}$ & $4.4410^{-4}$ & \\
\hline Bucket area & 5.013 & 23.631 & $\mathrm{eV}-\mathrm{s}$ \\
\hline Betatron tune $\nu_{\beta}$ & 214 & 214 & \\
\hline \multicolumn{4}{|l|}{ Beams } \\
\hline Bunch frequency & 53.105 & 53.105 & $\mathrm{MHz}$ \\
\hline Bunch filling & $90 \%$ & $90 \%$ & \\
\hline Number of bunches & 37152 & 37152 & \\
\hline Number per bunch $N_{b}$ & $2.610^{10}$ & $2.610^{10}$ & \\
\hline rms bunch area & 0.4 & 2.00 & eV-s \\
\hline rms bunch length $\sigma_{\ell} / \sigma_{\tau}$ & $6.03 / 0.201$ & $6.55 / 0.219$ & $\mathrm{~cm} / \mathrm{ns}$ \\
\hline rms energy spread $\sigma_{\delta}$ & $6.3110^{-4}$ & $1.4610^{-4}$ & \\
\hline \multicolumn{4}{|l|}{ Freq. at bunch mode } \\
\hline$m=0, f_{(0)}$ & 0.507 & 0.467 & $\mathrm{GHz}$ \\
\hline$m=1, f_{(1)}$ & 1.015 & 0.934 & $\mathrm{GHz}$ \\
\hline$m_{\mathrm{eff}}=0.304, f_{\left(m_{\mathrm{eff}}\right)}$ & 0.169 & 0.156 & $\mathrm{GHz}$ \\
\hline
\end{tabular}


with $b=h$, while the transverse impedance is

$$
\left[Z_{V, H}\right]_{\mathrm{wall}}=\frac{2 R}{h^{2}}\left[\frac{Z_{\|}}{n}\right]_{\mathrm{wall}, \mathrm{cyl}} F_{V, H}
$$

with $|n|^{-1 / 2}$ replaced by $\left|n-\nu_{\beta}\right|^{-1 / 2}$. In above, the form factors are $F_{L}=0.938, F_{V}=0.821$, and $F_{H}=0.408$, for this particular elliptic beam pipe. These are computed for an aluminum beam pipe ( $\rho=2.6510^{-8} \Omega$-m at room temperatures) and a copper beam pipe ( $\rho=1.7010^{-8} \Omega$-m at room temperature). The results are tabulated in Table 18 .

Power dissipated at the wall of the beam pipe by $M$ bunches each containing $N_{b}$ protons is

$$
P=\Gamma\left(\frac{3}{4}\right) M\left[\frac{N_{b} e c}{2 \pi}\right]^{2}\left[\frac{Z_{0} \rho}{2}\right]^{1 / 2} \frac{1}{\sigma_{\ell}^{3 / 2} b},
$$

where $\Gamma\left(\frac{3}{4}\right)=1.225416702$ is the Gamma function at $\frac{3}{4}$, and the beam pipe has been approximated to be cylindrical with radius $b$. Note that the power loss is proportional to $\sigma_{\ell}^{-3 / 2}$ where $\sigma_{\ell}=\sigma_{\tau} c$ is the rms bunch length and $c$ the velocity of light. As is displayed in Table 18, the power loss in each aluminum beam beam pipe is 30 to $27 \mathrm{~kW}$ for injection to storage. For each copper beam pipe, the power loss is 24 to $21 \mathrm{~kW}$.

The resistive wall impedances of the beam pipe dominate because of its large size and small pipe radii. At a fixed frequency ${ }^{2}$ $\omega /(2 \pi)$, the wall impedances scale as

$$
\left[\frac{Z_{\|}}{n}\right]_{\text {wall }} \propto \frac{1}{h} \sqrt{\frac{R}{\omega}} \quad \text { and } \quad\left[Z_{\perp}\right]_{\mathrm{wall}} \propto \frac{1}{h^{3}} \sqrt{\frac{R^{3}}{\omega}} .
$$

where $R$ is the radius of the ring. On the other hand, the inductive parts of the impedances of the beam position monitors (BPMs), for example, scale as

$$
\left[\frac{\mathcal{I} m Z_{\|}}{n}\right]_{\mathrm{BPM}} \propto \frac{1}{\sqrt{R} \omega} \quad \text { and } \quad\left[Z_{\perp}\right]_{\mathrm{BPM}} \propto \frac{\sqrt{R}}{h^{2} \omega}
$$

where we have assume that the betatron tune and therefore the number of BPM sets scale as $\sqrt{R}$. The contributions of the BPMs are plotted alongside with the contributions of the resistive wall in Fig. 22. We see that the contributions of the BPMs are about an order of magnitude smaller than those of the resistive wall, even if the cold copper pipes are taken as reference.

\subsubsection{Potential-Well Distortion}

The bunch of the prescribed length $\sigma_{\tau}$ and energy spread $\sigma_{E}$ listed in Table 17 will match the rf bucket set up at the voltage of $V_{\text {rf }}=50 \mathrm{MV}$ (or $200 \mathrm{MV}$ for Stage 2 at storage). In the presence of an inductive impedance $\operatorname{Im} Z_{\|} / n$, the particles inside a bunch see an additional force proportional to the gradient of the particle distribution. Providing that the bunch is short, a beam particle at a time advance $\tau$ with respect to the synchronous particle sees a potential drop or voltage of

$$
V_{\text {ind }}=\frac{e N_{b}}{\sqrt{2 \pi} \omega_{0} \sigma_{\tau}^{2}} \frac{\mathcal{I} m Z_{\|}}{n} \frac{\tau}{\sigma_{\tau}}
$$

which is to be subtracted from the rf voltage supplied by the klystron. Thus, for an inductive impedance at the rf harmonic, this induced voltage counteracts the supplied rf voltage. The bunch shape will be distorted and its length increased. As an illustration, we evaluate this voltage distortion at the $95 \%$ of the bunch or $\tau=\sqrt{6} \sigma_{\tau}$. As shown in Table 18, this inductive voltage, $V_{\text {ind }}$, is less than $1 \mathrm{MV}$ for the aluminum pipe and copper pipe. Since the low-field beam pipe will be made of aluminum with or without an internal copper coating, the bunch shape will not be affected much by the inductive wall. In other words, potential-wall distortion is of no importance here.

\subsubsection{Longitudinal Mode-Coupling}

For only motion in the longitudinal phase space, mode $m=0$ represents static motion like the potential-well distortion that we discussed before. The next modes are the dipole mode $m=1$, quadrupole mode $m=2$, etc. In the presence of coupling

\footnotetext{
${ }^{2}$ Looking at a fixed revolution harmonic, the right side of each equation in Eqns (21) and (22) should be multiplied by a factor of $\sqrt{R}$.
} 

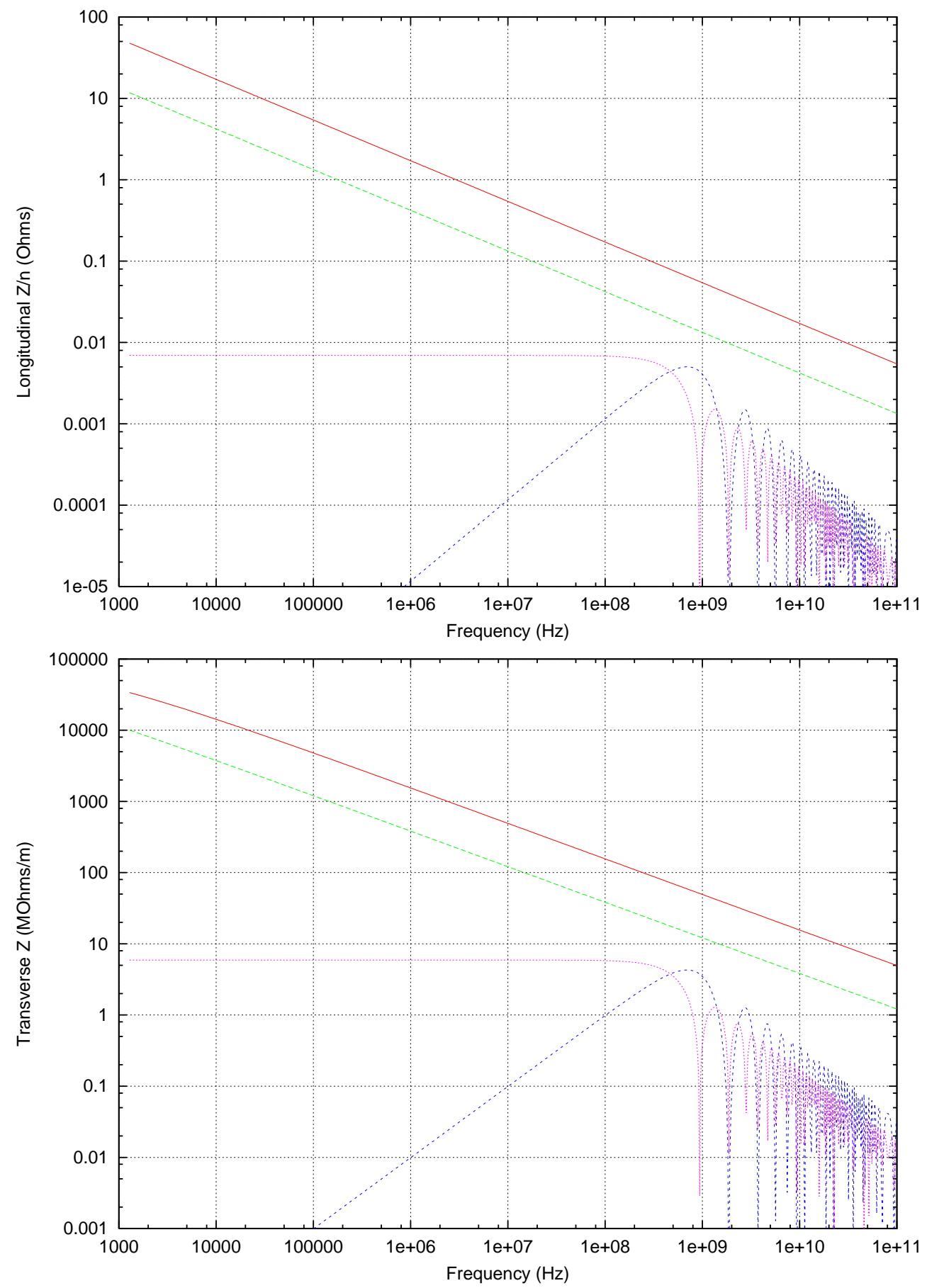

Figure 22: Longitudinal impedance $Z_{\|} / n$ (upper plot) and transverse impedance (lower plot). In each plot, from top: real or imaginary parts of resistive wall contribution for an aluminum beam pipe and a cold copper beam pipe. Lower curves: real and imaginary parts of the BPM contribution. All beam pipes are elliptical with half-axes $9 \mathrm{~mm}$ and $14 \mathrm{~mm}$. One set of BPM striplines, either horizontal or vertical, is assumed at each location of a FODO quadrupole. Each stripline is of length $8 \mathrm{~cm}$ subtending an angle of $30^{\circ}$ at the center of the pipe. 
Table 18: Collective instability limits of the low field VLHC bunches.

\begin{tabular}{|c|c|c|c|}
\hline & \multicolumn{2}{|c|}{ STAGE 1} & \\
\hline & Injection & Storage & \\
\hline Beam pipe radii $h / w$ & $9 / 14$ & $9 / 14$ & $\mathrm{~mm}$ \\
\hline Rf voltage $V_{\text {rf }}$ & 50 & 50 & MV \\
\hline Mode coupling limit $\left(Z_{\|} / n\right)_{\mathrm{eff}}$ & 6.26 & 8.05 & $\Omega$ \\
\hline Mode coupling limit $\left(Z_{\perp}\right)_{\text {eff }}$ & 112 & 576 & $\mathrm{M} \Omega / \mathrm{m}$ \\
\hline Resistivity of aluminum $\rho$ & $2.6510^{-8}$ & $2.6510^{-8}$ & $\Omega-\mathrm{m}$ \\
\hline Skin depth at $n=1, \delta_{1}$ & 0.23 & 0.23 & $\mathrm{~cm}$ \\
\hline Wall imp. at $n=1,\left[Z_{\|} / n\right]_{1}$ & 44.7 & 44.7 & $(1+j) \Omega$ \\
\hline$\left[Z_{\perp}\right]_{1}$ & $3.5610^{4}$ & $3.5610^{4}$ & $(1+j) \mathrm{M} \Omega / \mathrm{m}$ \\
\hline $\operatorname{Re}$ or $\operatorname{Im}\left(Z_{\|} / n\right)_{\text {wall }}$ at rf freq & 0.0736 & 0.0736 & $\Omega$ \\
\hline Voltage distortion & 0.869 & 0.735 & MV \\
\hline $\operatorname{Re}$ or $\operatorname{Im}\left(Z_{\|} / n\right)_{\text {wall }}$ at $m=1$ & 0.0505 & 0.0527 & $\Omega$ \\
\hline $\operatorname{Re}$ or $\operatorname{Im}\left(Z_{\perp}\right)_{\text {wall }}$ at $m_{\mathrm{eff}}$ & 98.5 & 103 & $\mathrm{M} \Omega / \mathrm{m}$ \\
\hline Power loss & 30.3 & 26.7 & $\mathrm{~kW}$ \\
\hline Multi-bunch growth time & 1.20 & 26.8 & turns \\
\hline Resistivity of copper $\rho$ & $1.7010^{-8}$ & $1.7010^{-8}$ & $\Omega-\mathrm{m}$ \\
\hline Skin depth at $n=1, \delta_{1}$ & 0.018 & 0.018 & $\mathrm{~cm}$ \\
\hline Wall imp. at $n=1,\left[Z_{\|} / n\right]_{1}$ & 35.8 & 35.8 & $(1+j) \Omega$ \\
\hline$\left[Z_{\perp}\right]_{1}$ & $28.510^{3}$ & $28.510^{3}$ & $(1+j) \mathrm{M} \Omega / \mathrm{m}$ \\
\hline $\operatorname{Re}$ or $\operatorname{Im}\left(Z_{\|} / n\right)_{\text {wall }}$ at rf freq & 0.0589 & 0.0589 & $\Omega$ \\
\hline Voltage distortion & 0.696 & 0.589 & MV \\
\hline $\operatorname{Re}$ or $\operatorname{Im}\left(Z_{\|} / n\right)_{\text {wall }}$ at $m=1$ & 0.0405 & 0.0422 & $\Omega$ \\
\hline $\operatorname{Re}$ or $\operatorname{Im}\left(Z_{\perp}\right)_{\text {wall }}$ at $m_{\mathrm{eff}}$ & 78.9 & 82.3 & $\mathrm{M} \Omega / \mathrm{m}$ \\
\hline Power loss & 24.3 & 21.4 & $\mathrm{~kW}$ \\
\hline Multi-bunch growth time & 1.5 & 33.4 & turns \\
\hline
\end{tabular}


impedance, the synchrotron sidebands are no longer equally separated with the $m$-th sideband at $\pm m \nu_{s} f_{0}$. The $m=2$ sideband will move towards the $m=1$ sideband as coupling impedance and/or bunch intensity increase. When the two sidebands merge into one, the $m=1$ and $m=2$ modes couple and an instability develops. This instability is not death threatening, stability will be regained after the bunch is lengthened and the energy spread increases. The threshold of the instability is given approximately by, for Gaussian distribution,

$$
\left[\frac{\mathcal{I} m Z_{\|}}{n}\right]_{\mathrm{eff}} \lesssim \frac{2 \pi|\eta| E \sigma_{E}^{2}}{e I_{\mathrm{pk}}}
$$

where $I_{\mathrm{pk}}=e N_{b} /\left(\sqrt{2 \pi} \sigma_{\tau}\right)$ is the bunch peak current, and the effective impedance is

$$
\left[\frac{\mathcal{I} m Z_{\|}}{n}\right]_{\mathrm{eff}}=\frac{\int d \omega \frac{\operatorname{Im} Z_{\|}}{\omega} \omega_{0} h_{m}(\omega)}{\int d \omega h_{m}(\omega)}
$$

and $h_{m}(\omega)$ the power spectrum of mode $m$ and $\int d \omega$ implies discrete summation over all the $m$-th synchrotron sidebands. The longitudinal mode-coupling stability limits are listed in Table 18 for various situations of operation. The instability will develop first near $m=1$. Thus we evaluate the resistive wall impedance at $f_{(1)}$, with results shown in Table 18 . We see that with an aluminum beam pipe, the impedance $Z_{\|} / n$ at $f_{(1)}$ in Stage 1 is only $0.051 / 0.053 \Omega$ at injection/collision, and is very much lower than the stability limit of 6.26/8.05 $\Omega$. Thus, no longitudinal mode-coupling instability will occur.

Let us understand how the stability limit scales as the size of the accelerator ring increases. Notice that $|\eta| \sim \nu_{\beta}^{-2}$ in a FODO lattice and therefore scales as $|\eta| \propto R^{-1}$. Therefore, when the impedance of the ring is dominated by the resistive wall, the microwave stability criterion in Eqn. (24) scales as, at a fixed frequency,

$$
R^{1 / 2} \lesssim \frac{A \sigma_{E}}{R I_{b}} \propto \frac{A \sigma_{E}}{N_{b}}
$$

where Eqn. (21) has been used. Thus the instability becomes worse as $\sqrt{R}$ as the size of the ring increases.

\subsubsection{Transverse Mode-Coupling}

With transverse motion, the $m=0$ is a valid mode, which describes the bunch making rigid dipole oscillations in the transverse plane. This corresponds to just the pure betatron sidebands. Driven by the transverse impedance, the betatron tune decreases and the pure betatron sideband moves towards its first lower $m=-1$ synchrotron sideband. An instability will develop when the two overlap, which we call transverse mode-coupling instability (TMCI). Unlike the longitudinal counterpart, this instability is devastating. The growth time is usually small.

For an average bunch current $I_{b}$, the threshold driving impedance is

$$
\left[\mathcal{I} m Z_{\perp}\right]_{\mathrm{eff}} \lesssim \frac{8 E \omega_{0}^{2} \nu_{\beta} \nu_{s} \sigma_{\tau}}{e I_{b} c}
$$

where the effective transverse impedance is

$$
\left[\operatorname{Im} Z_{\perp}\right]_{\mathrm{eff}}=\frac{\int d \omega \operatorname{Im} Z_{\perp}(\omega) h_{m}(\omega)}{\int d \omega h_{m}(\omega)}
$$

and $\int d \omega$ implies discrete summation over the $m=0$ synchrotron sidebands of the betatron tune lines (or just the betatron sidebands). These limits for various operations are listed in Table 18. It appears that the resistive wall impedance should be evaluated at frequency between $f_{(0)}$ and $f_{(1)}$, where the two modes collide. In fact, for a Gaussian bunch interacting with the resistive wall impedance, this effective frequency can be computed. Substituting the Gaussian spectrum and Eqn. (19) into Eqn. (28) gives

$$
\left[\mathcal{I} m Z_{\perp}\right]_{\mathrm{eff}}=\frac{\left[\mathcal{I} m Z_{\perp}\right]_{1}}{n_{\mathrm{eff}}^{1 / 2}}
$$

with

$$
n_{\mathrm{eff}}=\frac{4 \pi}{\omega_{0} \sigma_{\tau} \Gamma^{2}\left(\frac{1}{4}\right)} \quad \text { or } \quad f_{\mathrm{eff}}=\frac{2}{\sigma_{\tau} \Gamma^{2}\left(\frac{1}{4}\right)}=\frac{0.304}{2 \sigma_{\tau}}
$$


where $\Gamma\left(\frac{1}{4}\right)=3.63561$ is the Gamma function evaluated at $\frac{1}{4}$. This corresponds to $f_{\left(m_{\mathrm{eff}}\right)}$ with $m_{\mathrm{eff}}=0.304$ if Eqn. (15) is used. As an example, for an aluminum beam pipe the results as seen in Table 18 show that the impedance is $98.5 / 102.7 \mathrm{M} \Omega / \mathrm{m}$ at injection/collision, which is smaller than the stability limit of $125.7 / 643.9 \mathrm{M} \Omega / \mathrm{m}$. Thus, no TMCI will occur. Equation (27) is just an approximate way to determine the threshold of the instability by equating the shift of the $m=0$ mode by $\nu_{s}$, the synchrotron tune. A more exact method is to compute the eigenmodes of a number of modes near $m=0$ and $m=-1$ as a function of the beam current or coupling impedance. Blaskiewicz [20] made such a computation for the high-field ring at injection. On the other hand, the threshold given by Eqn. (27) is 33\% larger. This serves as a verification that Eqn. (27) will give a reasonably estimate. On the other hand, the more exact numerical solution may arrive at a lower threshold and easily push the bunches at injection to a TMCI.

As the size of the ring increases, the stability criterion of Eqn. (27) scales according to

$$
R^{3 / 2} \lesssim \frac{\sqrt{R} \nu_{s} \sigma_{\tau}}{N_{b}}
$$

Thus, TMCI becomes worse as $R$. Comparing with Eqn. (26), TMCI will have a lower threshold than the longitudinal microwave instability as the size of the ring increases.

\subsubsection{Coupled-Bunch Instability}

The revolution frequencies of the VLHC rings are only $1.29 \mathrm{kHz}$. Thus the transverse resistive wall impedance at the betatron sideband of lowest negative frequency becomes very large and will drive a coupled-bunch instability.

For $M_{s}$ identical equally spaced bunches in the ring, there are $\mu=0, \cdots, M_{s}-1$ transverse coupled modes when the center of mass of one bunch lags behind its predecessor by the betatron phase of $2 \pi \mu / M_{s}$. At the same time, each bunch can execute longitudinal motion with $m=0,1, \cdots$ nodes. The growth rate for the $\mu m$-th mode is

$$
\frac{1}{\tau_{\mu m}}=-\frac{1}{1+m} \frac{e M I_{b} c}{4 \pi \nu_{\beta} E} \sum_{k} \operatorname{Re} Z_{\perp}\left[\left(k M_{s}-\mu+\nu_{\beta}+m \nu_{s}\right) \omega_{0}\right] F_{m}^{\prime}\left(\omega \tau_{L}-\chi\right),
$$

where $M$ is the number of bunches. Strictly speaking Eqn. (32) is correct only if $M=M_{s}$ or if the bunches are equally spaced. According to the 9-bucket spacing, the VLHC rings will be only 90\% filled. Thus Eqn. (32) will not be an accurate description of the beam dynamics.

As the frequency $\omega \rightarrow \pm 0$, the real part of the resistive-wall impedance approaches first $\pm|\omega|^{-1 / 2}$, then $|\omega|^{-1}$ when the skin depth exceeds the thickness of the pipe wall, and finally zero when the frequency is exactly zero. Therefore, there is always a mode $\mu$ that corresponds to a large negative $\operatorname{Re} Z_{\perp}$ and drives the transverse coupled-bunch instability. For example, with the betatron tune $\nu_{\beta}=214.4$, mode $\mu=215$ or frequency $-0.6 \omega_{0} /(2 \pi)=0.776 \mathrm{kHz}$ with $k=0$ in the summation of Eqn. (32) contributes the largest negative $\operatorname{Re} Z_{\perp}$, which is $-5.6310^{4} \mathrm{M} \Omega / \mathrm{m}$ if the Stage 1 beam pipe is of aluminum. Here, we stick to the $\pm|\omega|^{-1 / 2}$ dependency of the resistive wall impedance for simplicity, although at such a low frequency, the thickness of the beam pipe wall may be thinner than the skin depth. The next contribution with $k=1$ will give $\operatorname{Re} Z \perp=+227 \mathrm{M} \Omega / \mathrm{m}$ which is negligibly small compared with the contribution at harmonic -0.6 , which therefore dominates the contribution.

The growth rates turn out to be extremely fast in all situation. The $e$-folding growth time is 1.2/27 turns for Stage 1 at injection/storage, where aluminum pipes are considered. Fortunately, the frequency of this mode is very low, around $1 \mathrm{kHz}$. A damper can be easily designed.

As the size of the accelerator ring increases, it is easy to show that the growth time in revolution turns scales as

$$
\text { Growth time in turns } \propto \frac{N_{b}}{R},
$$

which explains why this transverse coupled-bunch instability driven by the resistive wall is a concern for the VLHC.

\subsubsection{Longitudinal Head-Tail}

Longitudinal head-tail instability has been observed in the Tevatron and we would like to examine its effect in the VLHC. This instability is a result of the asymmetric dependence of the momentum compaction factor $\alpha=\alpha_{0}\left(1+\alpha_{1} \delta\right)+\cdots$ on the momentum deviation $\delta$. Thus, a bunch will have slightly different length and therefore lose energy differently in the upper and lower halves of the longitudinal phase space. The growth rate of the synchrotron oscillation amplitude is given by

$$
\frac{1}{\tau}=-\frac{f_{0}}{2} \frac{d U}{d \sigma_{\tau}} \frac{\sigma_{\tau}}{E} \chi,
$$


where the energy loss per particle per turn is

$$
U\left(\sigma_{\tau}\right)=e^{2} N \int d \omega|\tilde{\rho}(\omega)|^{2} \operatorname{Re} Z_{0}^{\|}(\omega),
$$

and

$$
\chi=\frac{\alpha_{0}\left(\alpha_{1}-\eta+\frac{3}{2}\right)}{\eta} \approx \alpha_{1}+\frac{3}{2}
$$

denotes the asymmetry, which is roughly 2 since $\alpha_{1} \approx \frac{1}{2}$ for a FODO lattice.

The instability can be driven by a sharp resonance like the fundamental mode of the rf cavities. For the Tevatron, the quality factor $Q \approx 7000$ and the shunt impedance is $R_{s}=1.2 \mathrm{M} \Omega$. Here, for the VLHC, it is reasonable to assume $Q$ to be the same while $R_{s} / Q=1710$, about 10 times larger. Thus, the growth rate of Eqn. (34) scales as

$$
\frac{1}{\tau} \propto \frac{1}{R E} \propto \frac{1}{R^{2}} .
$$

Thus, we expect the growth rate to be very small for the VLHC. As shown in Table 19, these growth rates are very much less than $86400^{-1}=1.1510^{-5} \mathrm{~s}^{-1}$, implying that there will not be any appreciate growth of the synchrotron amplitude in a day. The small growth rates are a result of the low rf frequency and short bunch length, which represents a point on the upper plot of Fig. 23.to the very left of the $\left.-d U / d \sigma_{\tau}\right) \sigma_{\tau}$ peak.

The longitudinal head-tail instability can also be driven by a broad-band resonance of the coupling impedance. If we assume $Z_{0}^{\|} / n \approx 1$ does not change much with the size of the ring, $R_{s} / Q$ will increase as the radius $R$ of the ring. Thus, the growth rate driven by the broad-band impedance scales as

$$
\frac{1}{\tau} \propto \frac{1}{E} \propto \frac{1}{R},
$$

and will dominate the growth driven by the sharp resonance of the rf cavities. The lower plot in Fig. 23 gives the reduced differential energy loss, $\left(d U / d \sigma_{\tau}\right) \sigma_{\tau}$ as a function of $f_{r} \sigma_{\tau}$ with $f_{r}$ denoting the frequency of the broad-band.

Table 19: Parameters of the VLHC ring in Stage 1 and Stage 2, at injection and at storage.

\begin{tabular}{lccc}
\hline & \multicolumn{3}{c}{ STAGE 1 } \\
& Injection & Storage & \\
\hline Driven by rf resonance & & & \\
$f_{\mathrm{rf}}=477.9 \mathrm{MHz}, R_{s} / Q=1710 \Omega, Q=7000$ & & \\
Growth rates & $7.7710^{-6}$ & $3.8910^{-7}$ & $\mathrm{~s}^{-1}$ \\
\hline Driven by broad-band with $R_{s} / n=1.0 \Omega$ & & & \\
Growth rates $\left(f_{r}=3 \mathrm{GHz} Q=1\right)$ & $1.8410^{-3}$ & $6.4410^{-5}$ & $\mathrm{~s}^{-1}$ \\
Growth rates $\left(f_{r}=2 \mathrm{GHz} Q=1\right)$ & $2.7210^{-3}$ & $9.5410^{-5}$ & $\mathrm{~s}^{-1}$ \\
Growth rates $\left(f_{r}=1 \mathrm{GHz} Q=1\right)$ & $3.9610^{-3}$ & $1.5510^{-4}$ & $\mathrm{~s}^{-1}$ \\
Growth rates $\left(f_{r}=1 \mathrm{GHz} Q=2\right)$ & $2.8510^{-3}$ & $1.0910^{-4}$ & $\mathrm{~s}^{-1}$ \\
\hline Driven by resistive wall & & & \\
Resistivity of aluminum $\rho$ & & & \\
Growth rate & $2.6510^{-8}$ & $2.6510^{-8}$ & $\Omega-\mathrm{m}$ \\
& $4.6310^{-5}$ & $1.8410^{-6}$ & $\mathrm{~s}^{-1}$ \\
Resistivity of copper $\rho$ & & & \\
Growth rate & $1.7010^{-8}$ & $1.7010^{-8}$ & $\Omega-\mathrm{m}$ \\
\hline
\end{tabular}

The growth rates driven by a broad-band impedance at various frequencies are listed in Table 19. For the injection into the low-field rings, all growth rates from the table exceed $3600^{-1}=2.7810^{-4} \mathrm{~s}^{-1}$ or with growth time less than an hour. Unfortunately, the injection into the low-field rings is slow and is about an hour. Thus there will be significant growth of the synchrotron amplitude and thus the bunch area. 

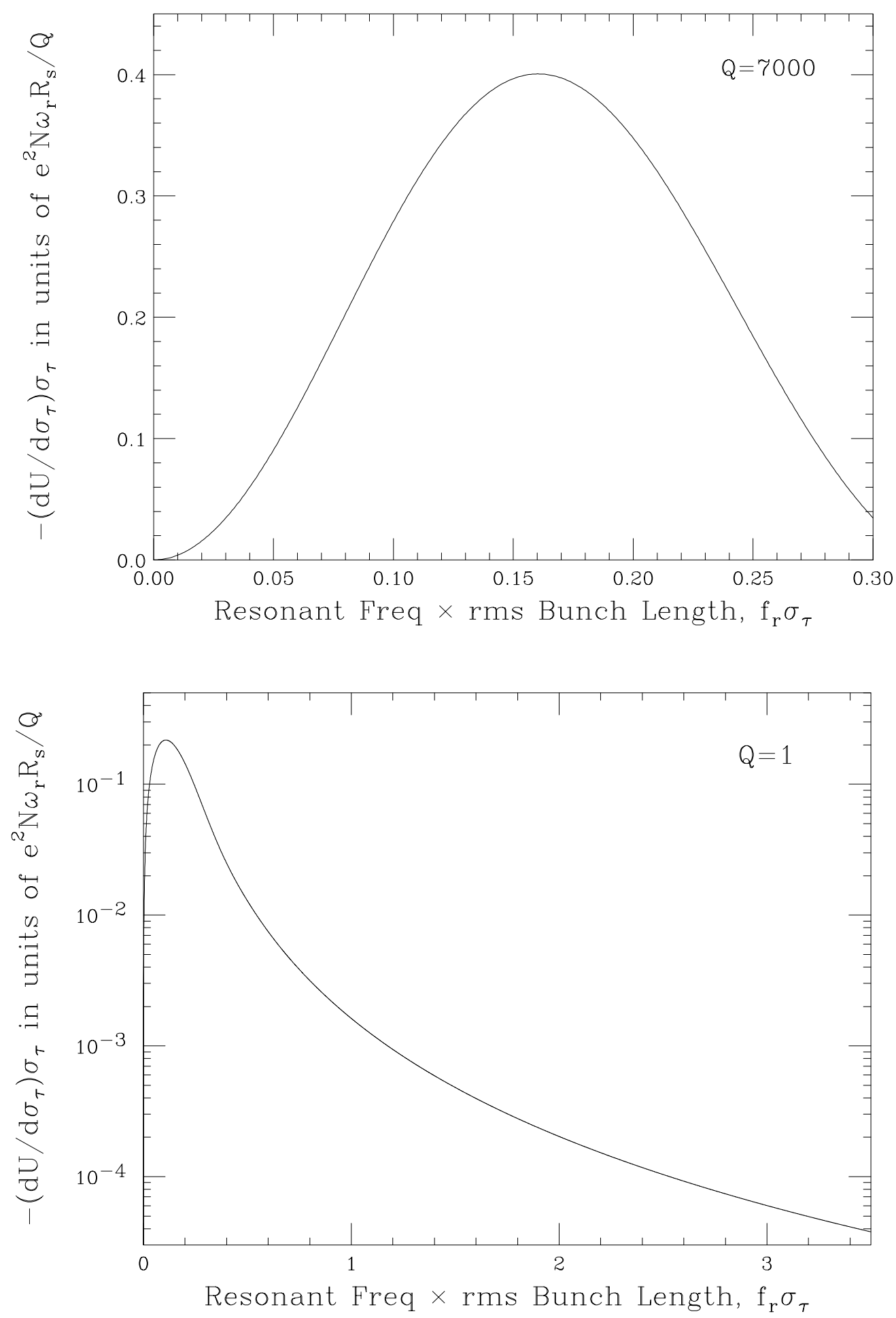

Figure 23: Plot of differential bunch energy loss $\left(d U / d \sigma_{\tau}\right) \sigma_{\tau}$ versus $f_{r} \sigma_{\tau}$ due to a sharp resonance (upper) or a broad-band impedance (lower). 
At storage, the growth rate is $6.4410^{-4} \mathrm{~s}^{-1}$ when $f_{r}=3 \mathrm{GHz}$ and $Q=1$, or growth time of 4.31 hours. This number appears to be insignificant for a store that lasts up to 10 hours. However, if the resonant frequency is reduced, the growth rate will increase, which corresponds to moving towards the peak of the $-\left(d U / d \sigma_{\tau}\right) \sigma_{\tau}$ plot from the right side in the lower plot of Fig. 23. As indicated in Table 19, when $Q=1$ and $f_{r}=1 \mathrm{GHz}$, the growth time reduces to 1.79 hours and becomes significant. It is also shown in the table that the growth rate will drop if the quality factor $Q$ increases.

The longitudinal head-tail instability can also be driven by the resistive wall impedance. The differential energy loss in Eqn (36) integrates to

$$
\frac{d U}{d \sigma_{\tau}} \sigma_{\tau}=-\frac{3 \Gamma\left(\frac{3}{4}\right)}{8 \pi^{2}} \frac{e^{2} N_{b}\left[\operatorname{Re} Z_{0}^{\|}\right]_{1}}{\omega_{0}^{1 / 2} \sigma_{\tau}^{3 / 2}}
$$

where $\left[\operatorname{Re} Z_{0}^{\|}\right]_{1}$ is the resistive part of the wall impedance at revolution frequency. The results in Table 19 show that this instability is insignificant. For example, at the injection of Stage 1, the growth time for the aluminum pipe is longer than the one hour injection time. At storage, the growth time is tens of hours. However the growth rate is inversely proportional to $\sigma_{\tau}^{3 / 2}$. Therefore, when the bunch length is further shortened in future design or operation, longitudinal head-tail instability driven by the resistive wall impedance may become important.

\subsubsection{Laslett Tune Shifts and Other Effects}

We have not studied all types of instability. There are many bunches in the collider ring. Therefore, coupled-bunch instabilities in both the longitudinal and transverse planes by sharp resonances can be serious, and an investigation is required. The beam pipe has a small bore. As a result, the image contribution to the coherent and incoherent betatron tune shifts can be important.

The very low revolution frequency leads to a variation in the magnetic image Laslett tune shift when the ring is partially filled during the injection process. The variation is due to the fact that the revolution period is comparable to the magnetic diffusion time through the beam pipe. Initial estimates of the latter effect produce tune shift variations of order 0.3 along the bunch train for a half filled ring. Increasing the vacuum pipe thickness and/or radius could reduce both of these problems. Increasing the dipole magnet gap from $20 \mathrm{~mm}$ to $28 \mathrm{~mm}$ reduces the DC Laslett tune shift from 1.0 to 0.6. The variation in tune along the bunch train could be reduced by quadrupoles running at multiples of the revolution frequency. It is envisioned, however, that injecting beam in an appropriately symmetric manner, with bunches entering the ring far apart from each other and gradually filling in between the already injected bunches, the tune variations can be alleviated.

\subsubsection{Bunch Coalescence}

The threshold of transverse mode coupling instability (TMCI) for the low-field VLHC has been computed by Burov et al., [21] using a matrix approach by including 5 radial and 5 azimuthal modes and also particle tracking. The same threshold has also been estimated by equating the downshift of the dipole betatron frequency to a synchrotron frequency. The latter gives a threshold about $40 \%$ higher than the Burov's result. One source of discrepancy is related to the fact that the transverse coupling impedance driving the instability is dominated by the resistive wall rather than a broad band. The transverse resistive wake goes to infinity as the inverse square root of the distance between the source and the test particles, and this distance has been taken as the rms bunch length in the estimation using the simple downshift formula. On the other hand, the matrix approach brings up numerical convergence questions from the divergence of the wake at small distances. This discrepancy is very important for the low-field VLHC, because the threshold from Burov's matrix calculation at injection with the nominal set of parameters is only about half the designed intensity.

With the danger of TMCI and the inability to estimate an accurate theoretical threshold, it is advisable to divide the bunch into a number of less intense bunches at injection and perform a coalescence at a higher energy. Below, we estimate the rf voltage required for the coalescence.

\section{Coalescence}

A low-field VLHC bunch has an intensity of $2.610^{10}$ particles in a $\omega_{\mathrm{rf}} /(2 \pi)=478.0 \mathrm{MHz}$ rf bucket with rf harmonic $h=371520$, corresponding to a ring circumference of $C=233.037 \mathrm{~km}$. Assume that this bunch is divided into 5 bunches at consecutive buckets at injection. The coalescence operation consists of the following steps:

(1) The coalescence is to be performed at $20 \mathrm{TeV}$ when the rf voltage is $V_{\mathrm{rf}}=50 \mathrm{MV}$. The parameter lists supplied by Foster [22] call for a rms bunch area of $2.0 \mathrm{eV}$-s at storage. We therefore assume the rms bunch area of each bunch before coalescence be $\sim 2.0 / 5=0.4 \mathrm{eV}$-s, or a total bunch area $A \sim 2.4 \mathrm{eV}$-s. The half energy spread and half width of the bunch 
are, respectively,

$$
\begin{gathered}
\left(\frac{\Delta E}{E}\right)_{1}=\sqrt{\frac{\omega_{s 1} A}{\pi|\eta| E}}=1.6010^{-4}, \\
(\Delta \tau)_{1}=\sqrt{\frac{|\eta| A}{\pi E \omega_{s 1}}}=0.239 \mathrm{~ns},
\end{gathered}
$$

where $\omega_{s 1} /(2 \pi)$ is the synchrotron frequency at the rf voltage $V_{1}=50 \mathrm{MV}$ and $\eta=2.1910^{-5}$ is the slip factor.

(2) The rf voltage $V_{\mathrm{rf}}$ is snapped down to $V_{2}$ so that the bucket height is equal to the energy spread of the bunch. We have

$$
\frac{V_{2}}{E}=\frac{\pi|\eta| h}{2}\left(\frac{\Delta E}{E}\right)_{1}^{2}=3.2310^{-7}
$$

or $V_{2}=6.47 \mathrm{MV}$.

(3) The bunch is allowed to rotate $90^{\circ}$ with synchrotron frequency

$$
\frac{\omega_{s 2}}{2 \pi}=\sqrt{\frac{|\eta| h e V_{2}}{2 \pi E}} \frac{\omega_{0}}{2 \pi}=0.835 \mathrm{~Hz},
$$

where $\omega_{0} /(2 \pi)=1.29 \mathrm{kHz}$ is the revolution frequency. The half energy spread becomes

$$
\left(\frac{\Delta E}{E}\right)_{2}=\frac{\omega_{s 2}}{|\eta|}(\Delta \tau)_{1}=5.7410^{-5}
$$

(4) The rf is then turned off and the coalescence rf with a frequency of $478.0 / 9=53.11 \mathrm{MHz}\left(h_{c}=h / 9\right)$ is turned on. The 5 bunches are rotated in the longitudinal phase space by $90^{\circ}$ and the $478.0 \mathrm{MHz}$ rf is then switched on to capture these bunches into one bucket. For total capture, the half energy spread $(\Delta E / E)_{2}$ of the bunches must rotate to a half width $(\Delta \tau)_{c}$ less than $k$ times half a rf wavelength $\pi / \omega_{\mathrm{rf}}$, where $k<1$. Notice that the phase equation of motion is

$$
\frac{d \tau}{d t}=\eta \frac{\delta E}{E}
$$

We therefore require

$$
(\Delta \tau)_{c}=\frac{|\eta|}{\omega_{s c}}\left(\frac{\Delta E}{E}\right)_{2} \lesssim \frac{k \pi}{\omega_{\mathrm{rf}}}
$$

where

$$
\omega_{s c}=\omega_{0} \sqrt{\frac{|\eta| h_{c} e V_{c}}{2 \pi E}}
$$

is the synchrotron frequency driven by the $53.11 \mathrm{MHz}$ rf system at voltage $V_{c}$. The result is

$$
\frac{e V_{c}}{E} \gtrsim \frac{h^{2}|\eta|}{2 \pi k^{2} h_{c}}\left(\frac{\Delta E}{E}\right)_{2}^{2}=\frac{1.5310^{-7}}{k^{2}},
$$

or $V_{c} \gtrsim 3.05 k^{-2} \mathrm{MV}$. The half energy spread of the coalesced bunch will be roughly 5 times the original multiplied by the factor $k^{2}$, or

$$
\left(\frac{\Delta E}{E}\right)_{c} \approx 5 k^{2}\left(\frac{\Delta E}{E}\right)_{2}=2.8710^{-4} k^{2} .
$$

The voltage of the captured if at $h=9 h_{c}$ must be at least

$$
V_{4}=\frac{\pi h|\eta| E}{2 e}\left(\frac{\Delta E}{E}\right)_{\text {coales }}^{2}=\left(\frac{5 k \pi}{6}\right)^{2} V_{c}=20.9 k^{2} \mathrm{MV} .
$$

The rf voltage is then raised to $50 \mathrm{MV}$ adiabatically, so that the coalesced bunch, having a rms bunch area of roughly, $2 \mathrm{eV}-\mathrm{s}$ will have the designed bunch length for collision. 


\section{An alternate method}

In Step 3 of the coalescence, the synchrotron frequency is $0.834 \mathrm{~Hz}$. Thus it takes $0.30 \mathrm{~s}$ for the bunch to rotate $90^{\circ}$ and spread out. It is important to check whether longitudinal microwave instability will develop or not in this long duration. At the end of the spread, the bunch occupies the whole width of the bucket, which is $C /(c h)=2.09 \mathrm{~ns}$, or a rms length of $\sigma_{\tau} \approx 0.427 \mathrm{~ns}$. The coalesced bunch should have a designed intensity of $2.610^{10}$ particles. Before coalescence, the intensity of each bunch is $1 / 5$ of it, or $N_{b}=0.5210^{10}$, corresponding to a peak current $I_{\mathrm{pk}}=e N_{b} /\left(\sqrt{2 \pi} \sigma_{\tau}\right)=0.77 \mathrm{~A}$. The Krinsky-Wang instability threshold is [23]

$$
\frac{Z_{0}^{\|}}{n}=\frac{2 \pi|\eta| E \sigma_{E}^{2}}{e I_{\mathrm{pk}}}=12 \Omega .
$$

The resistive wall impedance for the VLHC beam pipe composing of stainless steel was found to be only $\operatorname{Re} Z_{0}^{\|} / n=0.389 \Omega$ at $\mathrm{rf}$ frequency. [24] We can therefore conclude that longitudinal microwave instability will not occur.

Since we are far away from this instability, instead of snapping, one may also choose to reduce the $478.0 \mathrm{MHz}$ rf voltage adiabatically in Step 2 so that the bunch fills the whole bucket. This method will lead to a lower energy spread before coalescence, and therefore a lower coalescence voltage in the $53.11 \mathrm{MHz}$ rf. The lowest rf voltage required is

$$
\frac{V_{2}}{E}=\frac{\pi|\eta| h}{2}\left(\frac{h \omega_{0} A}{8 E}\right)^{2}=4.0310^{-8}
$$

or $V_{2}=0.8064 \mathrm{MV}$. The half energy spread is the same as the bucket height, or

$$
\left(\frac{\Delta E}{E}\right)_{2}=\sqrt{\frac{2 V_{2}}{\pi h|\eta| E}}=5.6310^{-5} .
$$

Step 3 is no longer necessary. We go to Step 4 where the coalescence rf is turned on to

$$
\frac{e V_{c}}{E} \gtrsim \frac{9 h|\eta|}{2 \pi k^{2}}\left(\frac{\Delta E}{E}\right)_{2}^{2}=\frac{1.4710^{-7}}{k^{2}}
$$

or $V_{c} \gtrsim 2.94 k^{-2} \mathrm{MV}$. The half energy spread is now

$$
\left(\frac{\Delta E}{E}\right)_{c} \approx 5 k^{2}\left(\frac{\Delta E}{E}\right)_{2}=2.8210^{-4} k^{2}
$$

The voltage of the captured if at $h=9 h_{c}$ must be at least

$$
V_{4}=\frac{\pi h|\eta| E}{2 e}\left(\frac{\Delta E}{E}\right)_{\text {coales }}^{2}=\left(\frac{5 k \pi}{6}\right)^{2} V_{c}=20.1 k^{2} \mathrm{MV}
$$




\subsection{Influence of Magnetic Fields on Damping Rates}

The variation of damping rates in a combined function machine was considered by A. Hofmann and B. Zotter [25]. The discussion here follows their treatment. Damping times in the three planes can be expressed in terms of the damping partition numbers $J_{x}, J_{y}, J_{s}$ as

$$
\tau_{x}=\frac{2 \tau_{0}}{J_{x}}, \tau_{y}=\frac{2 \tau_{0}}{J_{y}}, \tau_{s}=\frac{2 \tau_{0}}{J_{s}} ; \quad \tau_{0}=\frac{E}{U}
$$

Here $\tau_{x}, \tau_{y}, \tau_{s}$ are the amplitude damping times, $E$ is the particle energy and $U$ is the energy lost per turn to synchrotron radiation. The partition numbers are given by

$$
J_{x}=1-\frac{I_{4}}{I_{2}}, \quad J_{y}=1, \quad J_{s}=2+\frac{I_{4}}{I_{2}}
$$

where the synchrotron radiation integrals are

$$
\begin{aligned}
& I_{2}=\oint \frac{d s}{\rho^{2}} \\
& I_{4}=\oint\left[1+2 K \rho^{2}\right] \frac{D_{x}}{\rho^{3}} d s
\end{aligned}
$$

In separated function magnets, the second term in square brackets in Eq. (59) never contributes as $K \rho^{2}=0$ in both dipole and quadrupole magnets. In these magnets, $I_{4} \approx 0$ so $J_{x} \simeq 1, J_{s} \simeq 2$ and motion is damped in all three planes.

In a combined function magnet, the second term in Eq. (59) has a significant contribution. The straight sections do not contribute to the above integrals and we can estimate the integrals by the following sums over the $\mathrm{F}$ and $\mathrm{D}$ magnets,

$$
I_{4}=N\left[\left(1+2 K_{F} \rho_{F}^{2}\right) \frac{L_{F}}{\rho_{F}^{3}}\left\langle D_{x}^{F}\right\rangle+\left(1-2 K_{D} \rho_{D}^{2}\right) \frac{L_{D}}{\rho_{D}^{3}}\left\langle D_{x}^{D}\right\rangle\right]
$$

where $N$ is the number of $\mathrm{F}$ magnets (same as the number of D magnets), $K_{F}, K_{D}$ are the absolute values of the normalized gradients which in general may be unequal as can the bend radius $\rho_{F}, \rho_{D}$. Since $K \rho^{2} \gg 1$ in the arcs of the VLHC, we can write to a very good approximation

$$
I_{4} \simeq 2 N\left[\frac{K_{F} L_{F}}{\rho_{F}}\left\langle D_{x}^{F}\right\rangle-\frac{K_{D} L_{D}}{\rho_{D}}\left\langle D_{x}^{D}\right\rangle\right]
$$

In a combined function magnet, the average dispersions are given by

$$
\begin{aligned}
\left\langle D_{x}^{F}\right\rangle & =\frac{1}{\rho_{F} K_{F}}\left[\left(1+r_{\rho} r_{K}^{2}\right) \frac{G}{\theta_{F}}+1\right] \\
\left\langle D_{x}^{D}\right\rangle & =\frac{1}{\rho_{D} K_{D}}\left[\frac{\left(1+r_{\rho} r_{K}^{2}\right)}{r_{\rho} r_{K}} \frac{G}{\theta_{D}}-1\right]
\end{aligned}
$$

where

$$
\begin{aligned}
G & =\frac{\sin \theta_{F} \sinh \theta_{D}}{r_{K} \sin \theta_{F} \cosh \theta_{D}-\cos \theta_{F} \sinh \theta_{D}}, \\
\theta_{F, D} & =\frac{1}{2} L_{F, D} \sqrt{K_{F, D}}, \quad r_{\rho}=\frac{\rho_{F}}{\rho_{D}}, \quad r_{K}=\sqrt{\frac{K_{F}}{K_{D}}}
\end{aligned}
$$

Similarly

$$
I_{2}=N \frac{L_{F}}{\rho_{F}^{2}}\left(1+\frac{r_{K}^{2}}{r_{L}}\right), \quad r_{L}=\frac{L_{F}}{L_{D}}
$$

Combining Eqs. (66) and (61) together yields for the damping partition number

$$
J_{x}=-1-2 \frac{\left(1-r_{\rho}\right)\left(1+r_{\rho} r_{K}^{2}\right)}{1+r_{\rho}^{2} / r_{L}} \frac{G}{\theta_{F}}
$$




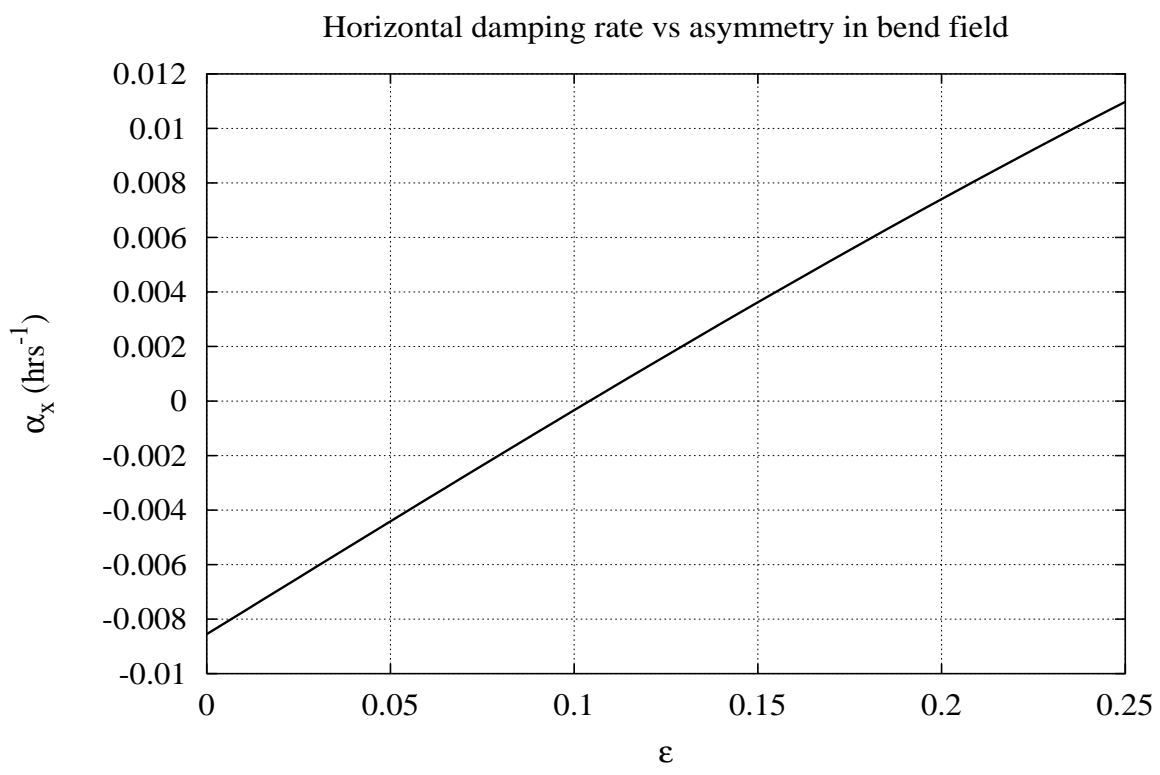

Figure 24: Damping rate in $1 / \mathrm{hrs}$ as a function of the bend field asymmetry $\epsilon$. The motion is just damped when the asymmetry is about $10 \%$.

One consequence of this relation is that in a ring where the $\mathrm{F}$ and $\mathrm{D}$ magnets have the same bend strength $\rho_{F}=\rho_{D}$ or $r_{\rho}=1$, we have

$$
J_{x}=-1
$$

Thus the horizontal motion is always anti-damped in such a ring irrespective of the lengths and gradients $K_{F}, K_{D}$ of the magnets.

The above general expression for $J_{x}$ can be simplified for the VLHC where we assume (i) equal phase advances in both planes so that $r_{L} r_{K}^{2}=K_{F} L_{F} /\left(K_{D} L_{D}\right)=1$ and (ii) equal lengths of the $\mathrm{F}$ and $\mathrm{D}$ magnets so that $r_{L}=1$ which then implies equal gradients $K_{F}=K_{D}$. In this case,

$$
J_{x}=-1+2 \frac{\left(r_{\rho}-1\right)\left(r_{\rho}+1\right)}{1+r_{\rho}^{2} / r_{L}} \frac{G}{\theta_{F}}
$$

The phase advance per cell $\mu_{C}$ is related to $\theta_{F}$ generally as

$$
\cos \mu_{C}=\cos 2 \theta_{F} \cosh 2 \theta_{D}+\frac{1-r_{K}^{2}}{2 r_{K}} \sin 2 \theta_{F} \sinh 2 \theta_{D}
$$

Specializing to the case with $r_{K}=1$ and $\theta_{F}=\theta_{D}$,

$$
\cos \mu_{C}=\cos 2 \theta_{F} \cosh 2 \theta_{F}
$$

When the phase advance per cell is $\mu_{C}=\pi / 2$, this equation shows that $\theta_{F}=\pi / 4$ which implies that $G / \theta_{f}=2.426$.

We assume that only a small change in bend fields between the F and $\mathrm{D}$ magnets will be physically possible. Writing

$$
\rho_{F}=\rho_{0}(1+\epsilon), \quad \rho_{D}=\rho_{0}(1-\epsilon)
$$

where $\rho_{0}$ is the nominal bend radius, the horizontal damping rate as a function of the asymmetry parameter $\epsilon$ is

$$
\frac{1}{\tau_{x}}=\frac{1}{2 \tau_{0}}\left[-1+\frac{4 \epsilon}{1+\epsilon^{2}}(2.426)\right]
$$

Figure 24 shows the damping rate as a function of the bend field asymmetry $\epsilon$. In the nominal case with $\epsilon=0$, the horizontal anti-damping time is $\tau_{x}=117 \mathrm{hrs}$. A $\pm 10 \%$ change in the bend fields (the bend should be weaker where the dispersion is larger) is enough to convert this to just damped motion. Smaller than $10 \%$ changes can increase the anti-damping rate to a value where this does not cause any appreciable emittance growth over the length of a store - about 10 hours. The price to pay for this additional knob is that the F and D magnets have to be on separate buses. 


\subsection{Polarization}

Some interest exists within the particle physics community for colliding beam experiments with high energy polarized protons. (e.g. in [26]) The VLHC Stage $1 p p$-collider would offer a great opportunity to achieve polarized $p p$ collisions at or close to $\sqrt{s}=40 \mathrm{TeV}$.

In [27] it was shown that a Combined Function (CF) lattice provides a unique feature at high energy. For a CF lattice the envelope of the linear intrinsic spin-obit resonances (LISORs) decreases asymptotically with $1 / \sqrt{\gamma}$, where $\gamma$ is the Lorentz factor, whereas for a separated function (SF) lattice the envelope increases with $\sqrt{\gamma}$. This effect can be explained perturbatively by the rapid spin precession component around the vertical in the bending field of the CF magnets which cancels to a great extent the tilt away from the vertical due to the gradient field. In a SF lattice there is no bending field in the quadrupoles.

The decrease of the LISORs with energy is a 1-st order effect, but the LISORs are the largest contribution to the off-orbit T-BMT driving term and in the approximation of leading powers in $G \gamma[28,29](G=(g-2) / 2 \approx 1.79$ being the gyromagnetic anomaly of the proton), i.e. at high energy, they are the dominant contribution to the higher order (kinetic) spin orbit coupling.

The key concepts for polarization in the presence of spin orbit coupling are the Derbenev-Kondatenko $\hat{n}$-axis [30, 31] and the amplitude dependent spin tune $[32,31] \nu$. Given $P_{\text {lim }}$, the modulus of the average of $\hat{n}$ over an invariant torus described by the normalized action vector $\vec{J}$, the long term polarization average of a beam $P$ can be factorized [29] as $P=\int P_{\text {lim }}(\vec{J}) P_{\text {dyn }}(\vec{J}) d \vec{J}$, where $P_{\text {dyn }}$ is the average of the spin action $\hat{S} \cdot \hat{n}$ over the spins on each torus. $P_{\text {lim }}$ is completely determined by the lattice, the energy and $\vec{J}$, whereas $P_{\text {dyn }}$ depends on the "history" of the beam, i.e. the polarization at injection and the acceleration process. It has been shown [33] that $P_{\text {dyn }}$ is an adiabatic invariant, i.e. is hardly changed during sufficiently slow acceleration. $P_{\text {lim }}$ and the acceleration rate for preservation of $P_{\text {dyn }}$ are particularly low whenever the non-perturbative resonance condition $\nu(\vec{J}, \gamma)=k_{x} Q_{x}+k_{y} Q_{y}+k_{z} Q_{z}$ is (sufficiently closely) fulfilled (e.g. [34, 33, 29]). Here the $Q_{i}$ are the orbital tunes and $k_{i} \in \mathcal{Z}$.

Simulations using the code SPRINT [35] and an early version of the Stage 1 lattice [36] without $\beta$-squeeze showed that the strengths of the LISORs indeed decays approximately with $1 / \sqrt{\gamma}$ and that the highest resonance strengths are around 1.1 for purely vertical motion with $2.5 \sigma$ vertical amplitude (assuming $\epsilon_{\mathrm{rms}}=1.5 \pi \mathrm{mm} \mathrm{mrad}$ ). After choosing a suitable vertical tune (about 217.2862) and introducing 1, 3 or 5 identical pairs of Siberian Snakes with snake angle difference of $90^{\circ}$ in each pair (Lee-Courant schemes, e.g. [37]) distributed around the ring so that the on-orbit spin tune is $1 / 2$ independent of energy, we have simulated $P_{\text {lim }}$ and $\nu$ with up to $5 \sigma$ vertical orbital amplitude in 3 energy ranges (strong resonance around injection, strong resonance close to $20 \mathrm{TeV} \&$ region without strong resonances close to $20 \mathrm{TeV} \rightarrow$ potential working point). The simulations were performed using the SODOM-2 algorithm [38] implemented in SPRINT. The simulations so far did not show any higher order (kinetic) spin orbit resonances up to $4 \sigma$ with 6 snakes and up to $5 \sigma$ with 10 snakes. In a $10 \mathrm{GeV}$ range around the potential working point the spin tune spread was less than .15 and $P_{\lim }$ was more than $92 \%$ up to $5 \sigma$ with 6 snakes.

Further simulations, e.g. including horizontal and longitudinal amplitudes and misalignment, are of course necessary. Moreover, spin dynamics in the injection chain has to be studied and most likely modifications to the pre-accelerators will be necessary.

Our preliminary simulations suggest that the VLHC Stage 1 CF ring could be a unique opportunity to obtain polarized protons at high energy. If one wants to take this opportunity it is essential to make spin dynamics a design issue at an early stage of the project. 


\section{STAGE 2: THE HIGH-FIELD RING}

\subsection{Luminosity Versus Energy}

The high field VLHC ring will be the first cryogenic collider to operate in the synchrotron radiation dominated regime, in which the radiation damping time is shorter than the storage time. In this regime there are practical and economic limits to the cryogenic system which can be installed. There is a maximum value $P_{\text {cryo }}$ for the synchrotron radiation which can be absorbed in each ring. It is necessary to adjust the beam parameters to stay within the installed power limit, resulting in a maximum luminosity which depends on the beam energy $E$ according to

$$
L_{\text {max }} \propto \frac{P_{\text {cryo }}}{E}
$$

The exact version of this "maximum power law" is derived as follows.

The total number of protons burnt off per beam, in a store time of length $T_{\text {store }}$ in a collider with $N_{I P}$ interaction points, is

$$
N_{\text {burn }}=N_{I P} L_{\text {ave }} \sigma_{\text {tot }} T_{\text {store }}
$$

where $\sigma_{t o t}$ is the total cross section and $L_{a v e}$ is the average luminosity. The number of protons in each beam at the beginning of the store must be at least this large, so

$$
N M \geq N_{\text {burn }}
$$

where $N$ is the number of protons in each of the $M$ bunches. The total synchrotron radiation power per ring is

$$
P=\left(\frac{U_{0}}{T}\right) N M
$$

where $U_{0}$ is the energy radiated per proton per turn, and $T$ is the revolution period. The synchrotron radiation damping time is closely related,

$$
T_{0}=\left(\frac{T}{U_{0}}\right) E
$$

so that, simply

$$
P=\frac{N M E}{T_{0}}
$$

Putting all this together, and recognizing that the synchrotron power is limited

$$
P<P_{\text {cryo }}
$$

then the minimum synchrotron power is achieved when every single proton is burnt off, so that

$$
L_{\text {ave }}<\frac{P_{\text {cryo }}}{E}\left(\frac{1}{N_{I P} \sigma_{\text {tot }}}\right)\left(\frac{T_{0}}{T_{\text {store }}}\right)
$$

This is the exact form of the maximum power law.

Although there is a clear advantage in reducing the storage time, $T_{\text {store }}$ must remain significantly larger than $T_{\text {damp }}$ in order to take advantage of radiatively damped beam sizes. Other factors (such as the refill time) will also play a role in determining the optimum value of $T_{\text {store }}$, but is safe to estimate that

$$
\frac{T_{0}}{T_{\text {store }}} \sim 0.2
$$

Except for some uncertainty in this factor, the maximum power law clearly states that the installed capacity to absorb synchrotron radiation at cryogenic temperatures directly limits the attainable product of average luminosity and energy.

Similarly, if the beam stored energy per ring $U=N M E$ must be kept below a maximum value - for example if the beam dump has a limited capacity - then

$$
L_{\text {ave }}<\frac{U_{\max }}{E}\left(\frac{1}{N_{I P} \sigma_{\text {tot }}}\right)\left(\frac{1}{T_{\text {store }}}\right)
$$

Insofar as the stored energy is a practical limit to high field performance - to the product $L_{\text {ave }} E$ - then there is pressure to reduce $T_{\text {store }}$, and hence to increase the dipole field, to reduce the circumference, and to reduce the refill time. 


\begin{tabular}{|lrl} 
Storage energy, $E$ & 87.5 & $\mathrm{TeV}$ \\
Peak luminosity, $L_{\max }$ & $2 \times 10^{34}$ & $\mathrm{~cm}^{-2} \mathrm{~s}^{-1}$ \\
Inelastic cross section & 130 & $\mathrm{mbarn}$ \\
Total cross section & 169 & $\mathrm{mbarn}$ \\
Collision debris power, per IP & 73 & $\mathrm{~kW}$ \\
Dipole field at storage & 9.765 & $\mathrm{~T}$ \\
Distance from IP to first magnet & 30 & $\mathrm{~m}$ \\
Injection energy & 10 & $\mathrm{TeV}$ \\
Fill time & 30 & $\mathrm{~s}$ \\
Acceleration time & 2000 & $\mathrm{~s}$ \\
Synchrotron radiation damping time, $T_{0}$ & 2.48 & $\mathrm{~h}$ \\
Energy loss per turn, $U_{0}$ & 15.3 & $\mathrm{MeV}$ \\
Natural transverse emittance $(\mathrm{H})$ & .0397 & $\mu \mathrm{m}$ \\
Natural RMS momentum width & 5.5 & $10^{-6}$ \\
Number of bunches, $M$ & 37152 & \\
Fraction of buckets filled & 90 & $\%$ \\
Collision beta horz, $\beta_{x}^{*}$ & 3.7 & $\mathrm{~m}$ \\
Collision beta vert, $\beta_{y}^{*}$ & 0.37 & $\mathrm{~m}$ \\
Equilibrium emittance ratio, $\kappa$ & 0.1 & \\
& & \\
Initial bunch intensity, $N$ & 7.5 & $10^{9}$ \\
Protons per beam & 2.79 & $10^{14}$ \\
Beam current & 57.4 & $\mathrm{~mA}$ \\
Synch. rad. power, per beam $P$ & .88 & $\mathrm{MW}$ \\
Dipole linear heat load & 4.7 & $\mathrm{~W} / \mathrm{m}$ \\
Stored energy, per beam $U$ & 3.9 & $\mathrm{GJ}$ \\
& &
\end{tabular}

Table 20: Nominal parameters for stores in the high field ring.

\subsection{Operational Performance}

The high field ring will be the first hadron collider able to join all electron colliders in taking advantage of naturally flat beams. The instantaneous luminosity is

$$
\begin{aligned}
L & =\frac{M}{4 \pi T} \frac{N^{2}}{\sigma_{x}^{*} \sigma_{y}^{*}} \\
& =\frac{M}{4 \pi T} \frac{N^{2} \gamma}{\sqrt{\epsilon_{x} \epsilon_{y} \beta_{x}^{*} \beta_{y}^{*}}}
\end{aligned}
$$

where $T$ is the revolution period, and the flatness of the beam is conveniently measured by

$$
\kappa=\frac{\epsilon_{y}}{\epsilon_{x}}
$$

Figure 6 shows how the natural horizontal emittance varies with the arc half cell length, with a nominal value of $\epsilon_{\text {nat }} \approx 0.04 \mu \mathrm{m}$. If the linear coupling and the vertical dispersion in the arcs are both well controlled, and in the absence of stray sources of diffusion, the horizontal emittance will decrease to this value. The equilibrium vertical emittance can become much smaller the beams can become very flat. The high field ring should be able to achieve $\kappa \ll 0.1$, in common with conventional electron storage rings.

The total number of protons in the ring, $M N$, is approximately set by the need to provide enough for "luminosity burnoff", plus an overhead fraction that is dumped at the end of the store. Since the number of bunches $M$ is fixed, the need for a given peak luminosity then sets the single bunch population $N$. Nominal values for these and other parameters, including a conservative value of $\kappa=0.1$, are given in Table 20 . 
It is the head on beam-beam interaction which sets the minimum horizontal emittance, whether the beams are flat or round. Figure 25 shows the horizontal and vertical emittances decreasing to plateau values of $\epsilon_{x}$ and $\epsilon_{y}$ that are consistent with the beam-beam limit, and which are maintained by transverse beam heating. Figure 26 shows the corresponding evolution of instantaneous luminosity, and its average, during the store.

It is shown immediately below that, at the beam-beam limit, flat beams permit the product $\epsilon_{x} \epsilon_{y}$ to be greatly reduced, relative to round beams. Equation 85 shows that this then permits larger values for $\beta_{x}^{*}$ and $\beta_{y}^{*}$ at fixed luminosity. Flat beams permit more relaxed IR optics, even before the increased efficiencies of doublet optics are considered.

\subsubsection{The Head On Beam-Beam Interaction}

The horizontal and vertical tune shift parameters for bi-Gaussian beams are

$$
\xi_{x, y}=\frac{r}{2 \pi \gamma} \frac{N \beta_{x, y}^{*}}{\sigma_{x, y}^{*}\left(\sigma_{x}^{*}+\sigma_{y}^{*}\right)}
$$

where $r=1.535 \times 10^{-18} \mathrm{~m}$ is the classical radius of the proton. When the beams are round $\left(\beta_{x}^{*}=\beta_{y}^{*}, \epsilon_{x}^{*}=\epsilon_{y}^{*}\right)$ this reduces to

$$
\xi_{x}=\xi_{y}=\frac{r}{4 \pi} \frac{N}{\epsilon_{x}}
$$

with no dependence on $\gamma$ or $\beta^{*}$. By comparison, if the beams are very flat, $\kappa \ll 1$, then

$$
\xi_{x}=\xi_{y}=\frac{r}{2 \pi} \frac{N}{\epsilon_{x}}
$$

The horizontal and vertical beam-beam parameters are made equal in the flat beam case by asserting that the $\beta *$ ratio is also $\kappa$

$$
\kappa=\frac{\epsilon_{y}}{\epsilon_{x}}=\frac{\beta_{y}^{*}}{\beta_{x}^{*}}=\frac{\sigma_{y}^{*}}{\sigma_{x}^{*}}
$$

Equations 88 and 89 show that, whether the beam is round or flat, the beam-beam parameter depends only on the horizontal emittance $\epsilon_{x}$. Since the collision beta functions $\beta_{x}^{*}$ and $\beta_{y}^{*}$ do not influence the beam-beam parameters, they are adjusted (with a fixed ratio $\kappa$, and for given values of $M, N$, and $\epsilon_{x}$ ) in order to achieve the required peak luminosity.

The beam-beam limit is expected to be approximately the same for round or flat beams, given by

$$
\xi \leq 0.008
$$

This value is justified by practical experience at the SPS and at the Tevatron. The SPS operated at $\xi \approx 0.004$ (or slightly higher) with 6 collisions per turn. More recently, in Run Ib the Tevatron operated with $\xi \approx 0.0075$ with only 2 head on collisions per turn (as in the VLHC). Simulations predict that radiation damping might give the high field ring a slight additional advantage, which is by no means as strong as that commonly observed in electron storage rings. The numerical value of 0.008 is illustrated in Figure 27, which displays empirical data compiled by Keil and Talman for electron storage rings [39]. The "damping decrement" for the high field VLHC - the fraction of a damping period per head on collision - is approximately $10^{-7}$.

Equations 88 and 89 show that the minimum value of $\epsilon_{x}$ permitted by the beam-beam effect is the same for flat or round beams, within about a factor of 2. Then, re-writing Eqn. 85 as

$$
L=\frac{M}{4 \pi T} \frac{N^{2} \gamma}{\kappa \epsilon_{x} \beta_{x}^{*}}
$$

it is explicitly clear that with flat beams the value of $\beta_{x}^{*}$ can be increased by a factor of about $1 / \kappa$, a large amount and a significant advantage. Equation 90 then shows that the value of $\beta_{y}^{*}$ is about the same for flat or round beams.

Figure 28 shows how the horizontal and vertical beam-beam parameters evolve during a flat beam store. About $80 \%$ of the original beam is burned off after 20 hours. Store evolution parameters, including RF and longitudinal, are summarized in Table 21 . 


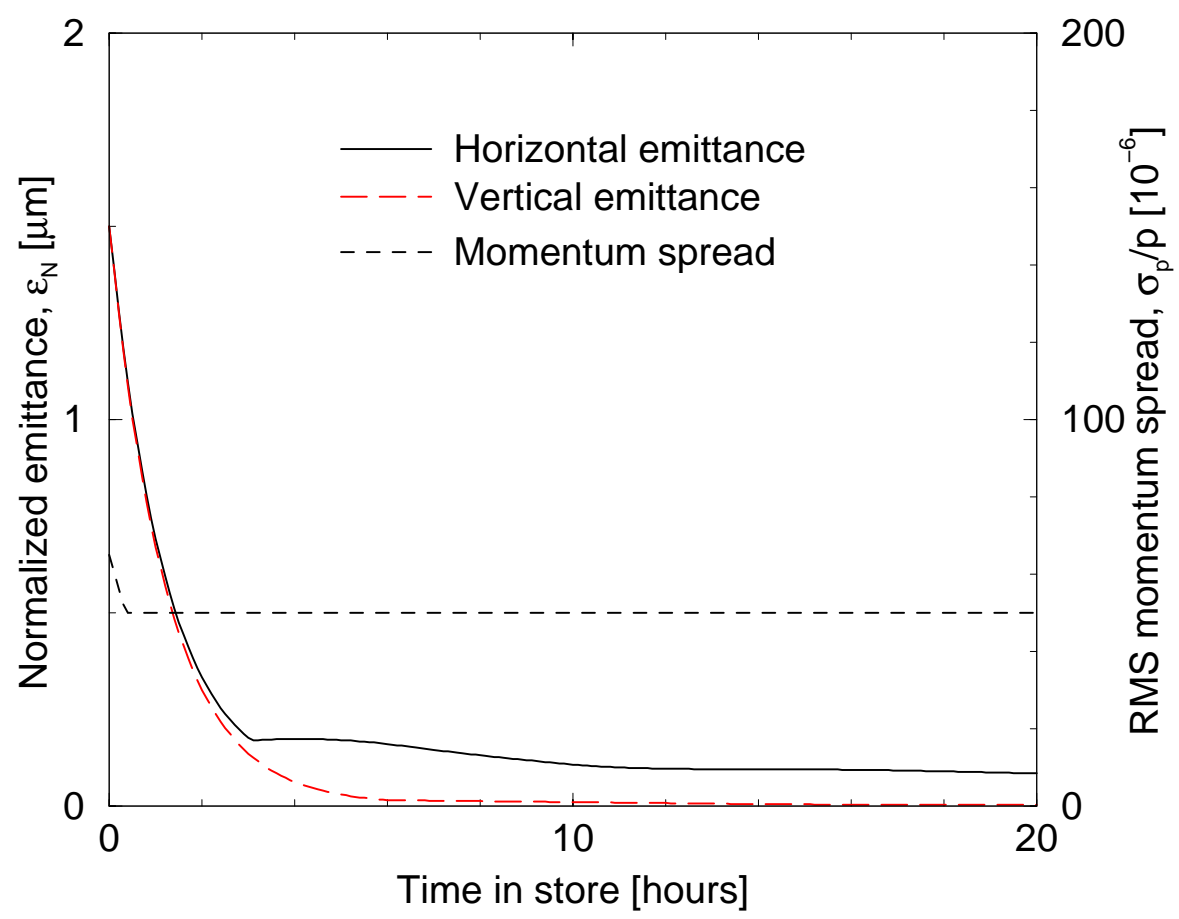

Figure 25: Evolution of the transverse emittances and the rms momentum spread during a store.

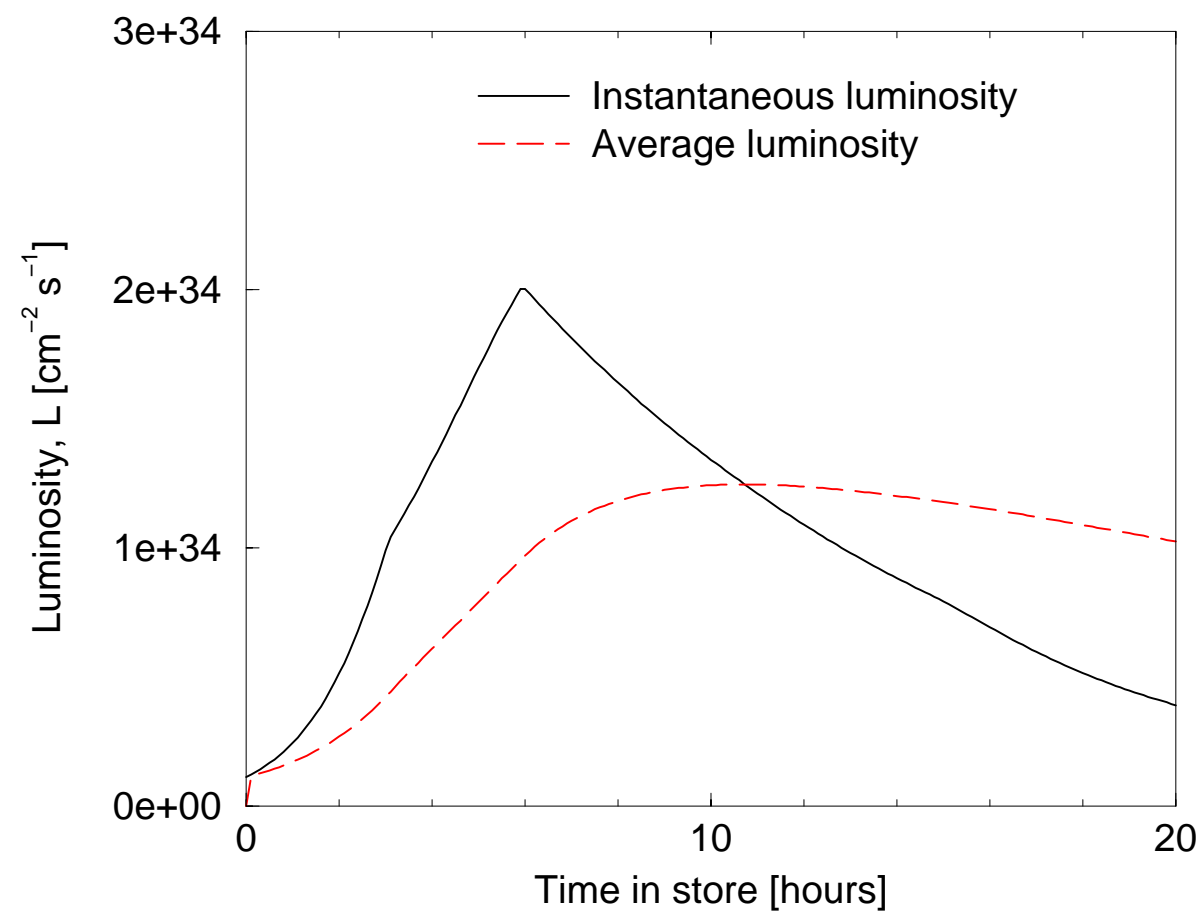

Figure 26: Evolution of the instantaneous and average luminosities, during a store. 


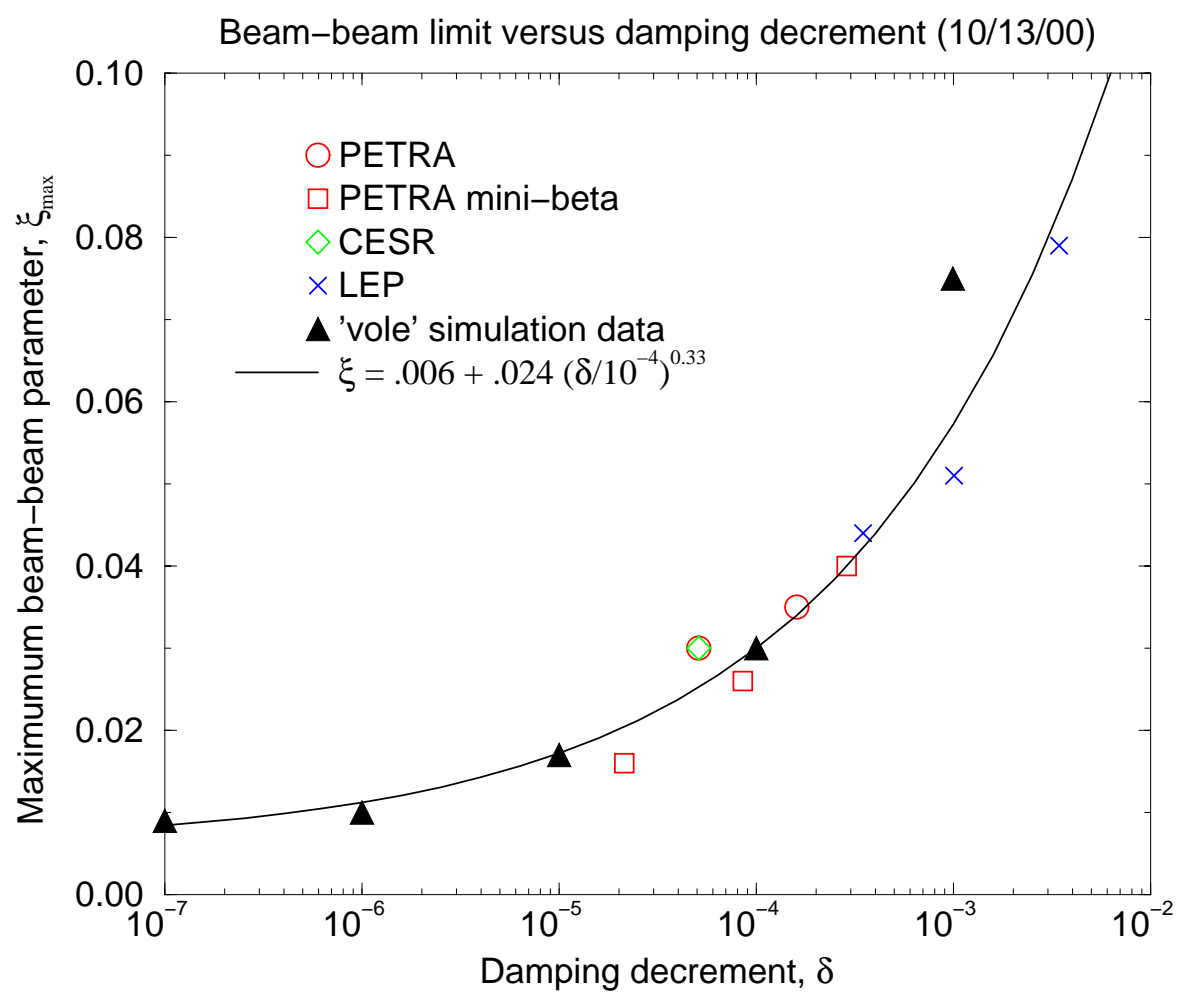

Figure 27: The beam-beam limit versus damping decrement in electron colliders, extrapolated by tracking towards VLHC parameters.

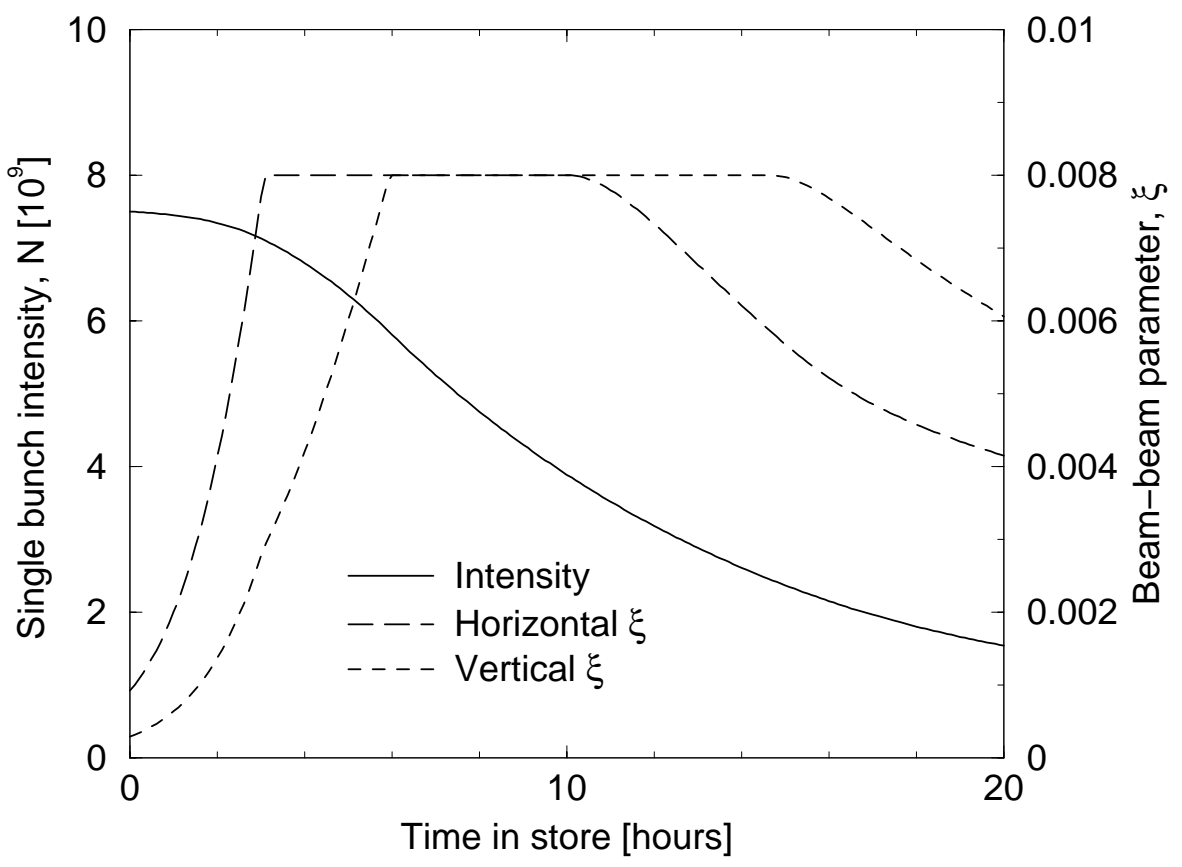

Figure 28: Evolution of beam-beam parameters, and the single bunch intensity, during the store. 


\begin{tabular}{|c|c|c|}
\hline \multicolumn{3}{|l|}{ INJECTION } \\
\hline Emittance, rms ( $\mathrm{H}$ and V) & 1.5 & $\mu \mathrm{m}$ \\
\hline Momentum width, rms & 233.1 & $10^{-6}$ \\
\hline Arc bunch size (betatron) & 254 & $\mu \mathrm{m}$ \\
\hline Arc bunch size (dispersion) & 330 & $\mu \mathrm{m}$ \\
\hline RF voltage & 50.0 & MV \\
\hline Longitudinal rms emittance & 2.0 & eV-s \\
\hline Longitudinal beta & 351 & $\mathrm{~m}$ \\
\hline Bunch length, rms & 81.9 & $\mathrm{~mm}$ \\
\hline Synchrotron tune & .00280 & \\
\hline Synchrotron frequency & 3.60 & $\mathrm{~Hz}$ \\
\hline \multicolumn{3}{|l|}{ STORE BEGINNING } \\
\hline Emittance, rms (H and V) & 1.5 & $\mu \mathrm{m}$ \\
\hline Momentum width, rms & 64.8 & $10^{-6}$ \\
\hline Arc bunch size (betatron) & 86 & $\mu \mathrm{m}$ \\
\hline Arc bunch size (dispersion) & 92.0 & $\mu \mathrm{m}$ \\
\hline Full crossing angle & 30.6 & $\mu \mathrm{rad}$ \\
\hline RF voltage & 200 & MV \\
\hline Longitudinal rms emittance & 2.0 & $\mathrm{eV}-\mathrm{s}$ \\
\hline Longitudinal beta & 520 & $\mathrm{~m}$ \\
\hline Bunch length, rms & 33.7 & $\mathrm{~mm}$ \\
\hline Synchrotron tune & .00189 & \\
\hline Synchrotron frequency & 2.44 & $\mathrm{~Hz}$ \\
\hline \multicolumn{3}{|c|}{ EARLY PLATEAU (Flat beams) } \\
\hline Beam-beam parameter & .008 & \\
\hline Emittance, rms (H) & .161 & $\mu \mathrm{m}$ \\
\hline Emittance, rms (V) & .016 & $\mu \mathrm{m}$ \\
\hline Momentum width, rms & 50.0 & $10^{-6}$ \\
\hline Arc bunch size (betatron) & 28.2 & $\mu \mathrm{m}$ \\
\hline Arc bunch size (dispersion) & 71.0 & $\mu \mathrm{m}$ \\
\hline IP bunch size $(\mathrm{H})$ & 2.53 & $\mu \mathrm{m}$ \\
\hline IP bunch size (V) & .25 & $\mu \mathrm{m}$ \\
\hline Full crossing angle & 10.0 & $\mu \mathrm{rad}$ \\
\hline RF voltage & 200 & MV \\
\hline Longitudinal rms emittance & 1.191 & eV-s \\
\hline Longitudinal beta & 520 & $\mathrm{~m}$ \\
\hline Bunch length, rms & 26.0 & $\mathrm{~mm}$ \\
\hline Synchrotron tune & .00189 & \\
\hline Synchrotron frequency & 2.44 & $\mathrm{~Hz}$ \\
\hline
\end{tabular}

Table 21: Store parameters, including longitudinal and RF. 


\subsubsection{Crossing Angles and Parasitic Beam-Beam Collisions.}

The horizontal and vertical tune shifts due to a single parasitic collisions are

$$
\Delta q_{x, y} \approx \pm \frac{r N}{2 \pi \gamma} \frac{\beta_{x, y}}{\Delta^{2}}
$$

where the approximation is valid if the full beam separation $\Delta$ is much greater than both horizontal and vertical beam sizes, so that the beam acts like a moving line charge. The tune shift is positive in the plane of the separation. The total separation increases linearly with respect to the distance $s$ from the IP, and is proportional to the total crossing angle $\alpha$, which is naturally scaled to the angular divergence of the beam by the factor $n$

$$
\Delta=\alpha s=n \sigma^{*} s \approx n \sigma
$$

This is illustrated in Figure 29, where the crossing angle $\alpha$ evolves to always keep the beams a constant number of horizontal sigmas apart. In the drift region next to the IP the horizontal and vertical angular divergences of the beam are equal, even for flat beams, since

$$
\frac{\sigma_{y}^{\prime *}}{\sigma_{x}^{\prime *}}=\sqrt{\frac{\epsilon_{y}}{\beta_{y}^{*}} \frac{\beta_{x}^{*}}{\epsilon_{x}}}=\sqrt{\frac{\kappa}{\kappa}}=1
$$

For parasitic collisions (except the first) the beams are essentially round, even with flat beams at the IP!

If the beams are thoroughly separated (into separate beam pipes, or with very large separations) at a distance $L_{\text {sep }}$ from the IP, then there are $4 L_{s e p} / S_{B}$ parasitic collisions around each IP, where $S_{B}$ is the longitudinal bunch separation. Many (or all) of these collisions occur in the drift region, where the beta functions are given by

$$
\beta=\beta^{*}+\frac{s^{2}}{\beta^{*}} \approx \frac{s^{2}}{\beta^{*}}
$$

and the approximation is valid when $s \gg \beta^{*}$ - that is, for all except perhaps the first parasitic collision. Putting all this together, the total tune shifts from all parasitic collisions in one interaction region are

$$
\Delta Q_{x, y} \approx \pm \frac{r N}{2 \pi \gamma} \frac{4}{S_{B}} \frac{L_{s e p}}{\beta_{x, y}^{*} \alpha^{2}}
$$

where the approximation is most valid if the beams are fully separated before the first IR quadrupole. The last term in this expression contains the optical variables of the most interest $-L_{s e p}, \beta^{*}$, and $\alpha$.

The horizontal beta function at each collision is much less than the vertical

$$
\frac{\beta_{x}}{\beta_{y}} \approx \frac{\beta_{y}^{*}}{\beta_{x}^{*}}=\kappa
$$

so that, taking Eqns 97 and 98 together gives

$$
\Delta Q_{x} \simeq-\kappa \Delta Q_{y}
$$

The horizontal tune shift is greatly suppressed with flat beams, to be much smaller than it is with round beams - if the values of $L_{s e p}$ and $\alpha$ compare favorably between the two cases.

Equation 97 shows that, if the beam separation plane is changed from horizontal to vertical with the total crossing angle $\alpha$ held fixed, then the long range tune shifts change sign with almost unchanged absolute values. If the separation plane is vertical at one IP, and horizontal at the other, then significant tune shift cancellations can be achieved. However, the beams are vertically separated in the arcs, no matter what the local crossing plane, because of the use of common coil magnet technology in the arc cells. Conversion from a horizontal beam separation plane to vertical separation in the arcs is not expected to be fundamentally difficult, even though the optical design of such a scheme has not yet been addressed. 


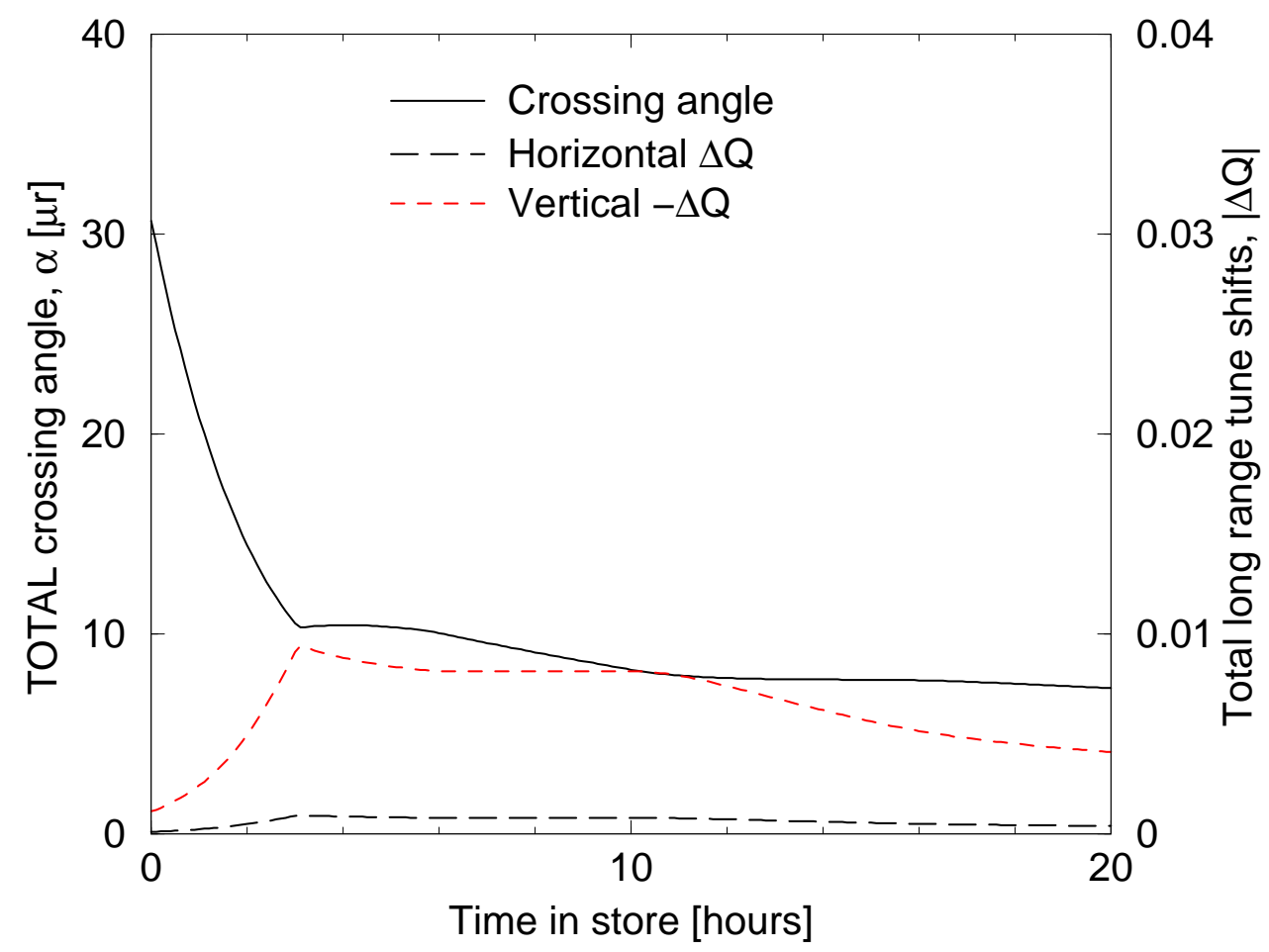

Figure 29: Evolution of the long range tune shifts, and the total horizontal crossing angle, during the store.

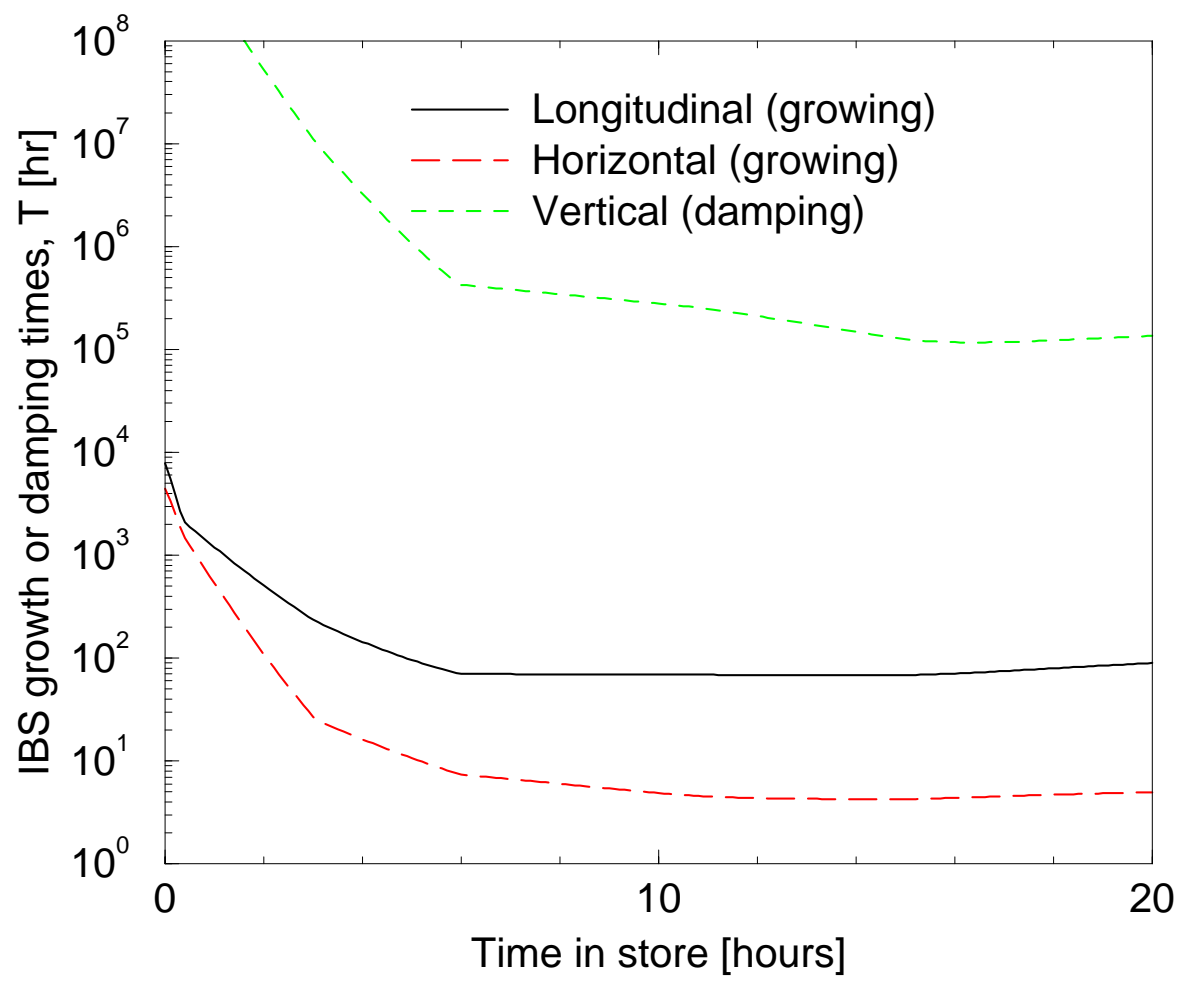

Figure 30: Intra beam scattering growth (or damping) times during the store. 


\subsubsection{Intra Beam Scattering and Diffusion}

Intra beam scattering (IBS) is an important effect in the high field ring, as illustrated for nominal store parameters in Figure 30. The horizontal growth rate is by far the strongest, with growth times of less than 10 hours after a couple of damping times, when the 6-dimensional phase space density is largest. This minimum growth time is controlled by heating the beams longitudinally, to prevent the rms momentum spread from falling below a minimum value of $\sigma_{p} / p$, as demonstrated in Figure 25. It also helps that, in order to respect the beam-beam limit, the horizontal emittance is heated to maintain a minimum value that is much larger than its natural value.

It is interesting to consider what fundamental limits IBS places on the flatness of the beam - how small can $\kappa$ be? Figure 31 shows how the minimum flatness depends on the momentum spread $\sigma_{p} / p$ which is maintained by longitudinal heating. The minimum value $\kappa_{\min }$ is defined as that value at which the horizontal IBS growth time is equal to the synchrotron radiation damping time. The results shown in Figure 31 assume that the RF voltage is held fixed. In contrast, Figure 32 show how the minimum flatness depends on the RMS bunch length $\sigma_{s}$, when the momentum spread $\sigma_{p} / p$ is held fixed. In this case the $\mathrm{RF}$ voltage changes significantly, scaling like $V_{r f} \sim 1 / \sigma_{s}^{2}$. Figure 33 shows the same two data sets, plotted as a function of the longitudinal emittance. It is clear that flatnesses as small as $\kappa \simeq 0.001$ are possible, from the perspective of our present theoretical understanding of IBS [40,41].

The relative paucity of world data on IBS results in cautious predictions, but it is reasonable to expect that IBS will be significant but not dominant in the high field ring of the VLHC. Fortunately, the copious data expected in the next few years from RHIC operations should greatly improve our understanding of the topic.

Any unwanted source of diffusion - whether IBS, power supply ripple, fundamental beam dynamics, or any unexpected source - could have a profound unwanted effect on the luminosity performance of the high field ring. A deep accelerator physics understanding of the spectrum of diffusion mechanisms is required for a confident high field design.

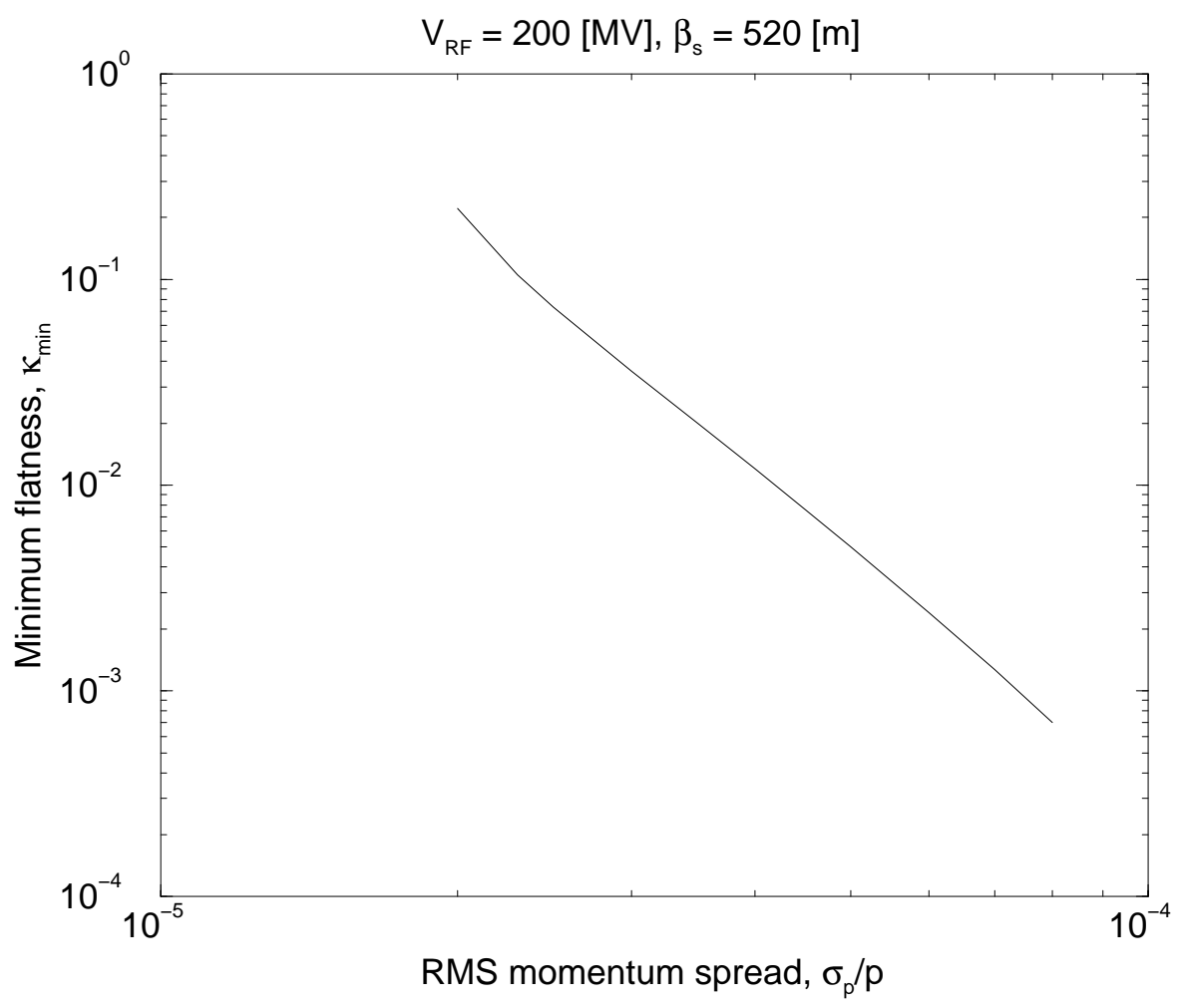

Figure 31: The effect of IBS on the minimum flatness $\kappa_{m i n}$, at fixed RF voltage $V_{R F}=200 \mathrm{MV}$, and $\beta_{s}=520 \mathrm{~m}$. 


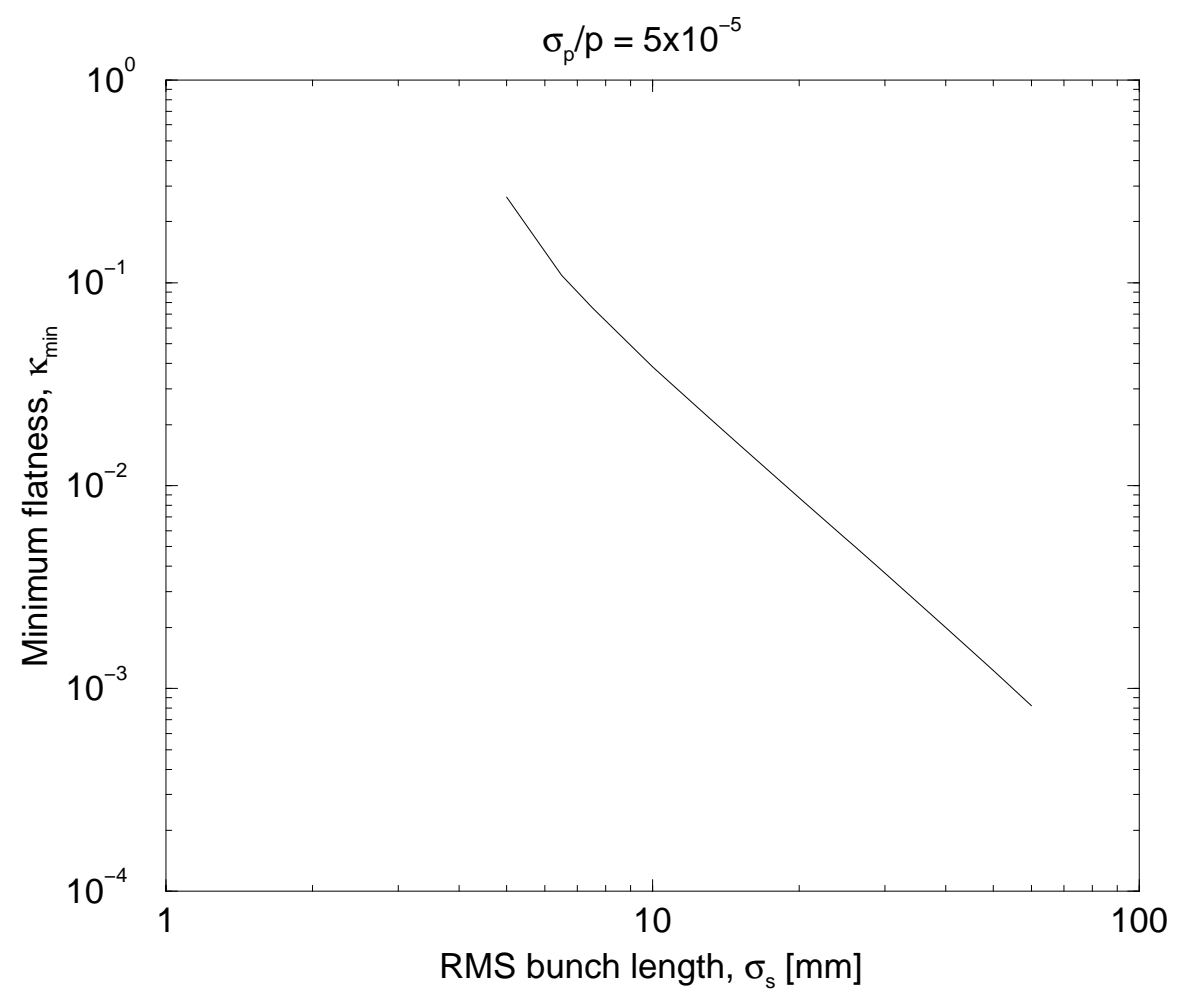

Figure 32: The effect of IBS on the minimum flatness $\kappa_{\min }$ at fixed momentum spread $\sigma_{p} / p=5 \times 10^{-5}$.

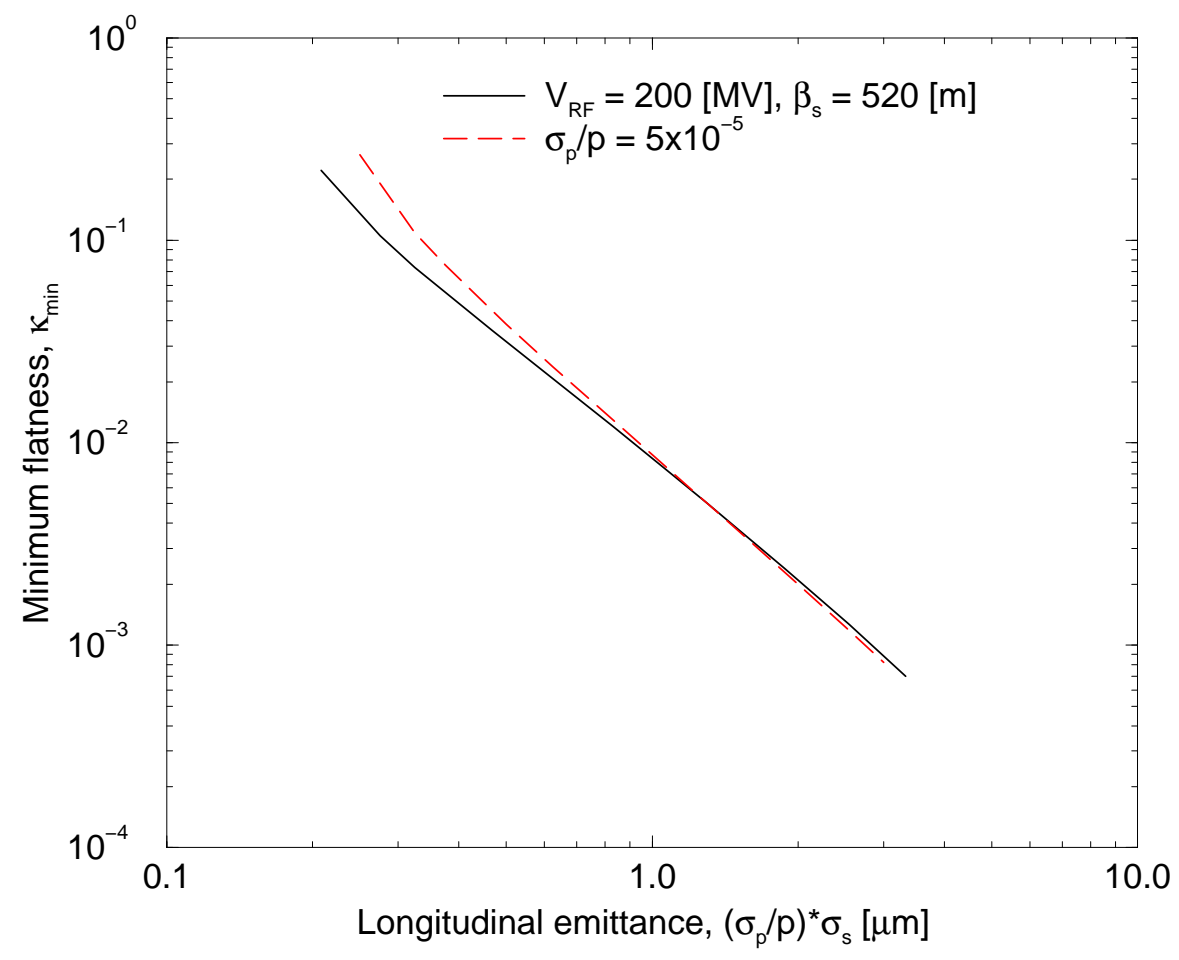

Figure 33: The minimum flatness $\kappa_{m i n}$ versus longitudinal emittance, $\left(\sigma_{p} / p\right) * \sigma_{s}$. The same data as in Figures 31 and 32. 


\subsection{Advantages and Disadvantages of Flat Beams}

All electron colliders, whether circular or straight, take advantage of flat beams and use doublet optics. However, it has never before been possible to use flat beams in a hadron collider. Flat beams have the advantages and disadvantages discussed below, which continue to be investigated. Should a major flaw be identified with flat proton beams, it is always possible to fall back to the conventional hadron solution of round beams and triplet optics.

Flat beams require the first quadrupole on both sides of the IP to be vertically focusing to both counter-rotating beams (whether the crossing angle is vertical or horizontal). Thus, the optics must be symmetric across the IP, and the first quadrupole must be a 2-in-1 magnet. This is unlike the conventional round beam triplet solution, in which the optics is usually (but not necessarily) anti-symmetric, and both beams pass through a single quadrupole bore. Because flat beams must enter separate quadrupole bores, there is a need for dipole beam separators inboard of the first quadrupole. On the one hand, this early separation is a relative disadvantage to doublet optics, since the first quadrupole is pushed further away from the IP. On the other hand, early separation is good for long range beam-beam effects, since there are (effectively) no parasitic collisions beyond the beginning of the first beam splitting dipole.

Table 22 compares the performance of flat and round beam in the high field ring. In both cases the beam-beam limit of $\xi=.008$ is reached 5 or 6 hours into the store, when the luminosity is at its peak. The horizontal and vertical emittances values recorded in Table 22 are those which are initially maintained (preventing further emittance shrinkage) when the horizontal and vertical beam-beam parameters first saturate. The same peak luminosity is achieved in both flat and round beam cases by adjusting the horizontal and vertical $\beta^{*}$ values.

\begin{tabular}{|lcc|}
\hline & FLAT & ROUND \\
& & \\
Flatness parameter, $\kappa$ & 0.1 & 1 \\
Beam-beam parameter $\xi_{x}=\xi_{y}$ & .008 & .008 \\
Peak luminosity $L\left(10^{34} \mathrm{~cm}^{-2} \mathrm{~s}^{-1}\right)$ & 2.0 & 2.0 \\
& & \\
Average luminosity, $20 \mathrm{hr} L_{\text {ave }}\left(10^{34} \mathrm{~cm}^{-2} \mathrm{~s}^{-1}\right)$ & 1.02 & 0.98 \\
Initial bunch intensity $N\left(10^{9}\right)$ & 7.5 & 7.5 \\
Collision beta horz $\beta_{x}^{*}(\mathrm{~m})$ & 3.7 & 0.71 \\
Collision beta vert $\beta_{y}^{*}(\mathrm{~m})$ & 0.37 & 0.71 \\
Maximum beta horz $\widehat{\beta_{x}}(\mathrm{~km})$ & 7.84 & 14.58 \\
Maximum beta vert $\widehat{\beta_{y}}(\mathrm{~km})$ & 10.75 & 14.58 \\
Horizontal emittance $\epsilon_{x}(\mu \mathrm{m})$ & .161 & .082 \\
Vertical emittance $\epsilon_{y}(\mu \mathrm{m})$ & .016 & .082 \\
Collision beam size horz $\sigma_{x}^{*}(\mu \mathrm{m})$ & 2.53 & 0.79 \\
Collision beam size vert $\sigma_{y}^{*}(\mu \mathrm{m})$ & 0.25 & 0.79 \\
Maximum beam size horz $\widehat{\sigma}_{x}(\mu \mathrm{m})$ & 116 & 113 \\
Maximum beam size vert $\widehat{\sigma}_{y}(\mu \mathrm{m})$ & 43 & 113 \\
Angular beam size horz $\sigma_{x}^{\prime}(\mu \mathrm{r})$ & 0.68 & 1.11 \\
Angular beam size vert $\sigma_{y}^{\prime}(\mu \mathrm{r})$ & 0.68 & 1.11 \\
& & \\
Total crossing angle $\alpha(\mu \mathrm{r})$ & 10.0 & 10.0 \\
Separation distance, $L_{s e p}(\mathrm{~m})$ & 30 & 120 \\
Number of long range collisions per IR & 20 & 84 \\
Long range tune shift per IR, horz $\left|\Delta Q_{x}\right|$ & .0008 & .0166 \\
Long range tune shift per IR, vert $\left|\Delta Q_{y}\right|$ & .0081 & .0166 \\
& & \\
\hline
\end{tabular}

Table 22: Flat and round beam performance parameters, quoted after about 6 hours at the "early plateau" just after peak luminosity, when the horizontal and vertical beam-beam parameters are both saturated.

A major advantage of flat beams comes from the order of magnitude increase of $\beta_{x}^{*}$, by a factor of about $1 / 2 \kappa$. Slightly offsetting this advantage, the vertical $\beta_{y}^{*}$ decreases by a factor of about 2 . Equations 88, 89, 90, and 92 between them explain why this is so - why collision optics are much more relaxed with flat beams. 
Another important advantage is that doublet optics lead to much lower value of $\widehat{\beta}$, the maximum beta in the IR quadrupoles. Lower $\widehat{\beta}$ means smaller beam sizes, and lower sensitivity to a host of dynamical effects. This reduction happens not just because $\beta_{x}^{*}$ is increased, but also because doublet focusing is inherently much more efficient than triplet focusing [42]. It can be understood heuristically by considering the first quad in a triplet to be present merely to split the horizontal and vertical beta functions, in order for the following two quads to act as a doublet. If the initial beta functions are already well separated - with flat beams - there is no need for the "beta splitting" first quadrupole. Because the "center of gravity" of a doublet is much closer to the IP than the equivalent triplet, then the maximum beta values $\widehat{\beta}_{x, y}$ are also much decreased. Early separation pushes the first quadrupole further from the IP, but the values of $\widehat{\beta}$ recorded in Table 22 still show the strong advantage of doublet optics.

Doublet collision optics contribute less to the natural linear chromaticities, and are gentler to the nonlinear chromaticities $[42,43]$. This leads to weaker chromaticity sextupoles in particular, and to better nonlinear dynamical behavior in general. The perturbation strength of an IR quad is often related to $K L \beta$, where $K$ is the quad strength and $L$ is the magnetic length. While $K$ is essentially constant between doublet and triplet optics, $L$ and $\beta$ are less with doublet optics, and the third quadrupole is absent.

Flat beams reduce both horizontal and vertical long range beam-beam tune shifts $\Delta Q_{x, y}$, with respect to the round beam alternative. Table 22 shows a factor of two reduction in the vertical, and an order of magnitude reduction of approximately $2 / \kappa$ in the horizontal. The value of the total crossing angle $\alpha$ is set to be identical with flat or round beams - and is independent of the plane of the crossing angle. Equations 97, 98, and 99 show that the long range tune shift reduction occurs in part because of the increased value of $\beta_{x}^{*}$, and in part because of the reduced value of $L_{s e p}$, with flat optics. There are far fewer parasitic collisions per interaction region with flat beams, than with round beams.

The most significant disadvantage is that the design of the first quadrupole is difficult. A 2-in-1 magnet with relatively close separation will not have as good field quality a single bore element. Nonetheless, the maximum beta $\widehat{\beta}$ is significantly less in the doublet design, so the tolerable field errors are larger. The preliminary reference harmonics listed in Table 62 are the subject of on-going Accelerator Physics and Magnet Physics evaluations. The beam size in the quadrupoles is quite small at collision energies ( $\widehat{\sigma} \sim 100 \mu \mathrm{m}$ in both cases) so field quality may not be as important as intuition based on previous experience suggests. It is reasonable to expect that dynamic stability with respect to closed orbit deviations becomes relatively much more important in this new parameter regime. Detailed tracking and simulation studies need to be performed to investigate these issues in detail.

Another difficulty is that neutral particles generated at the IP will aim head on for the center of the first 2-in-1 quadrupole. Each IR has $\sim 36 \mathrm{~kW}$ of beam garbage going off in each direction, and the neutrals carry about $30 \%$ (11 kW) of this power. Figures 40 and 54 shows a $5.5 \mathrm{~m}$ space in the lattice between the beam splitting dipole D1B and the first quadrupole Q1A, where an absorber can be placed to intercept this component and to shield the front face of the quad. The severity of this problem is difficult to quantify without a full blown energy deposition simulation of the beam losses, and a detailed draft design of the first quadrupole. Although these energy deposition simulations have begun (see below), it is clear that here, too, a detailed analysis is required.

A lack of energy flexibility may be a disadvantage to flat beams. The radiation damping time scales like $1 / E^{3}$, so if the collision energy is halved from $87.5 \mathrm{TeV}$ to $43.75 \mathrm{TeV}$, then the time increases to about 20 hours, and flat beams are no longer viable. If such a large dynamic range of collision energies is desired (at fixed geometry) then one possibility is to arrange the IR quadrupoles so that both doublet and triplet optics are possible. A lattice has been demonstrated in which 4 IR quadrupoles can swing either way - can act as a doublet or as a triplet [44].

An operational disadvantage is the need for careful tuning to keep the vertical emittance small. Electron rings routinely achieve beam flatnesses in the range $0.001<\kappa<0.01$ by controlling 2 linear quantities: the vertical dispersion in the arcs, and global betatron coupling. The mathematics behind the control room algorithms for such clean living is well understood, and shows no sign of significant differences between the electron and hadron colliders, except for the much different radiation damping times. It is not unreasonable for high field ring studies to also consider less conservative flatness values, of say $\kappa \approx 0.01$, which are closer to every day electron storage ring experience.

Figure 34 shows the evolution of a nominal round store, with the parameters listed in Table 22. It is to be compared with the equivalent flat beam performance displayed in Figures 25, 26, 28, 29, and 30. 

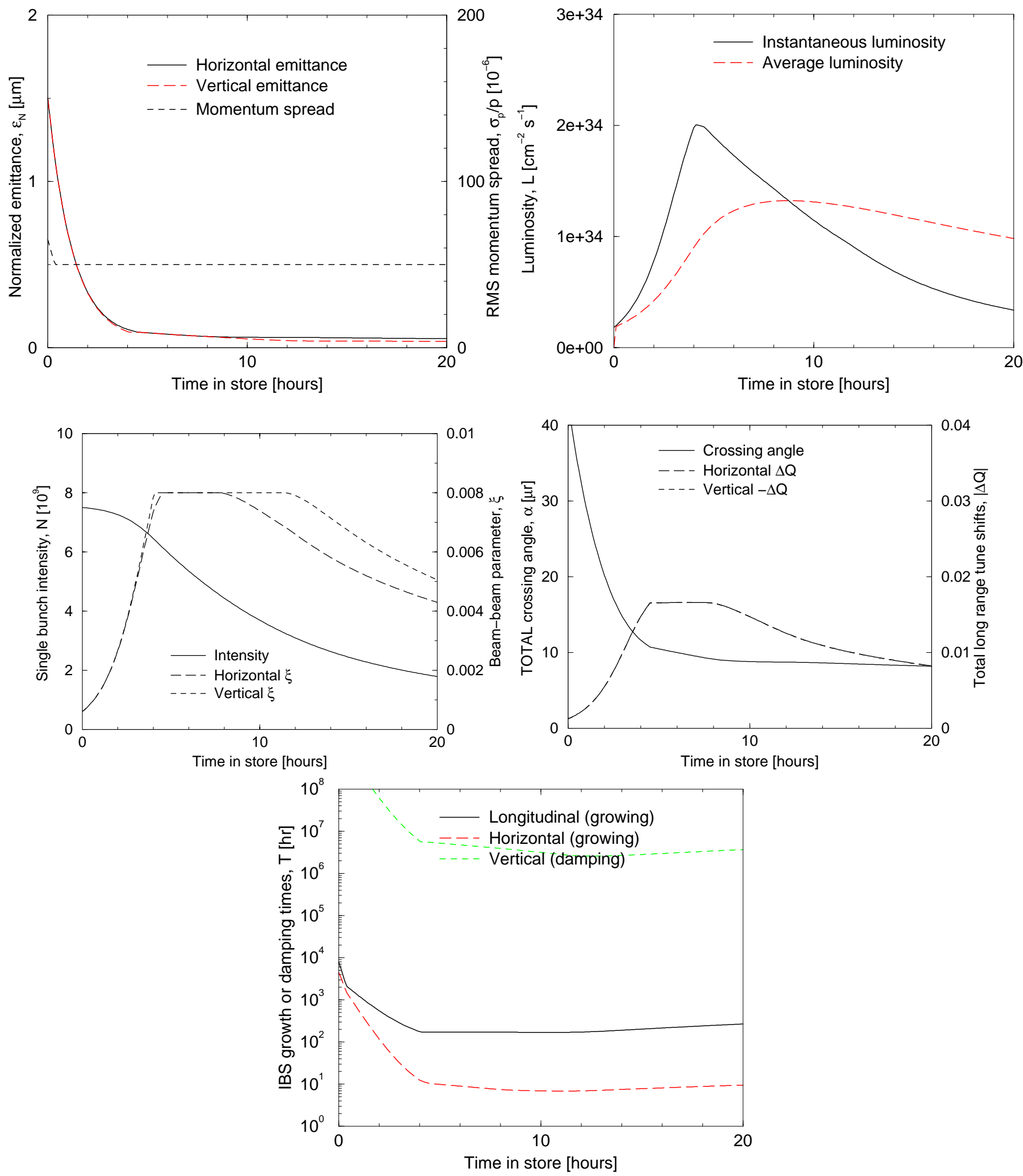

Figure 34: Round beam store evolution of the transverse emittances, rms momentum spread, instantaneous and average luminosities, beam-beam parameters, single bunch intensity, long range tune shifts, the total horizontal crossing angle, and IBS growth and damping times. 


\subsection{Lattice Optics}

The lattice for the high field (HF) ring is grouped into four units. The two arcs are joined with two cluster regions that contain dispersion suppressors, abort utility regions, interaction regions (IR), injection and extraction septa and kickers, et cetera. The lattice geometrically matches the low field (LF) ring lattice so that they both fit in the same tunnel. For convenience, a short list of some of the HF lattice parameters is shown in Table 23.

\begin{tabular}{|lrl|}
\hline & 218.19 & \\
Horizontal tune & 212.18 & \\
Vertical tune & 194.13 & \\
Transition gamma & 26.53 & $10^{-6}$ \\
Slip factor & 459 & $\mathrm{~m}$ \\
Maximum arc beta & 1.42 & $\mathrm{~m}$ \\
Maximum arc dispersion & 33.36 & $10^{4} \mathrm{Tm}$ \\
Rigidity at injection & 291.9 & $10^{3} \mathrm{Tm}$ \\
Rigidity at store & 0.37 & $\mathrm{~m}$ \\
Vertical $\beta^{*}$ (store) & 3.7 & $\mathrm{~m}$ \\
Horizontal $\beta^{*}$ (store) & .61 & $\mathrm{~km}$ \\
Maximum $\widehat{\beta}$, injection & 7.84 & $\mathrm{~km}$ \\
Maximum Horz $\widehat{\beta}$, store & 10.75 & $\mathrm{~km}$ \\
Maximum Vert $\widehat{\beta}$, store & & \\
\hline
\end{tabular}

Table 23: Short list of some high field lattice parameters.

The first major module to describe is the arc cell. The half cell length is $135.4865 \mathrm{~m}$. Both rings define optical modules with lengths in multiples of the half cell length. This is done to keep the geometries the same. Further requirements of the cell design are that the dipole magnetic length must be less than $17 \mathrm{~m}$, and that spool pieces are required for correctors and sextupoles. The arc quadrupole gradient is restricted to $400 \mathrm{~T} / \mathrm{m}$ and the maximum field of the dipole is $10 \mathrm{~T}$. Furthermore, dual plane Beam Position Monitors (BPM) are placed between the quadrupole and spool piece. Table 24 shows the parameters for the arc cell and for for the dispersion suppressor cell.

The other major arc module is the dispersion suppressor. There are two types, one bringing the dispersion to zero and the other matching the zero dispersion regions to arc cell Twiss function values. The dispersion suppressor follows the " $3 / 4,2 / 3$ " rule, in which the cell length is $3 / 4$ of the standard arc cell, and the bend angle is $2 / 3$ of the standard arc cell. This is used to achieve maximum packing fraction. These two dispersion modules only differ in their quadrupole strengths.

The layout and parameters for arc and dispersion suppressor cells are shown in Figures 35 and 36, and their Twiss functions in Figures 37 and 38.

There are two cluster regions. The one on the Fermilab site contains the abort insertion, Interaction Regions, et cetera. The off-site cluster region is simply filled with FODO cells, with and without dipoles, and dispersion suppressors. Figure 39 displays the Twiss functions for the off-site cluster region.

The IR gives $30 \mathrm{~m}$ free space from the interaction point to the first magnetic element. The first magnet encountered is a high field small bore magnet of $16 \mathrm{~T}$ field. This can handle the beams until they total separation becomes $8 \mathrm{~mm}$. Then the beams enter a lower field, $12 \mathrm{~T}$, magnet with a larger bore. These beam separation dipoles bend the beam vertically. This crossing region requires that the beam separation at the first quadrupole be $30 \mathrm{~mm}$. With these dimensions the first quadrupole is limited to $400 \mathrm{~T} / \mathrm{m}$ gradient. As the beams separate, a higher quadrupole field can be attained with a limit of $600 \mathrm{~T} / \mathrm{m}$. A schematic of this design is shown in Fig. 40.

With this design of the crossing region, an IR is designed to fit at two location in the on-site cluster region. The $\beta^{*}$ was varied from $0.37 \mathrm{~m}$ to $7.12 \mathrm{~m}$ (vertically, $\beta^{*}$ horizontally is 10 times larger). The maximum beta $\widehat{\beta}$ is $10.6 \mathrm{~km}$ with the collision optics and only about $760 \mathrm{~m}$ at injection. Figure 41 shows the $\beta^{*}$ squeeze every $0.25 \mathrm{~m}$. Figure 42 shows the injection and collision optics of the interaction region. Note that, due to the vertical crossing dipoles there is vertical dispersion throughout the ring. This will be dealt with as the design of the insertion progresses. Furthermore, the phase advance across the insertion has not been fixed. There are additional quads that can be varied to fix the phase advance. The abort region has yet to be designed. 


\begin{tabular}{|lrl|}
\hline & & \\
DIPOLE & 1.116 & $\mathrm{~T}$ \\
Field at injection & 9.765 & $\mathrm{~T}$ \\
Field at store & 29.887 & $\mathrm{~km}$ \\
Bend radius & 40 & $\mathrm{~mm}$ \\
Coil full width & 20 & $\mathrm{~mm}$ \\
Liner full width & .29 & $\mathrm{~m}$ \\
Vertical bore separation & 828 & $\mathrm{~kJ} / \mathrm{m}$ \\
Stored energy (2 bores) & & \\
& & \\
ARC CELLS & 24 & \\
Half cell harmonic & 135.486 & $\mathrm{~m}$ \\
Half cell length & 3.875 & $\mathrm{mrad}$ \\
Half cell bend angle & 1568 & \\
Half cell count & 7 & \\
Dipoles per half cell & 10976 & \\
Dipole count, total & 16.546 & $\mathrm{~m}$ \\
Dipole magnetic length & 85.5 & $\%$ \\
Dipole fill factor & 8.066 & $\mathrm{~m}$ \\
Quad magnetic length & 385.4 & $\mathrm{~T} / \mathrm{m}$ \\
Quad field gradient & & \\
& & \\
DISPERSION SUPPRESSOR CELLS & 18 & \\
Half cell harmonic & 101.614 & $\mathrm{~m}$ \\
Half cell length & 2.583 & $\mathrm{mrad}$ \\
Half cell bend angle & 80 & \\
Half cell count & 5 & \\
Dipoles per half cell & 400 & \\
Dipole count, total & 15.443 & $\mathrm{~m}$ \\
Dipole magnetic length & 76.0 & $\%$ \\
Dipole fill factor & 10.775 & $\mathrm{~m}$ \\
Quad magnetic length & 288.7 & $\mathrm{~T} / \mathrm{m}$ \\
Quad field gradient (QD1) & 376.2 & $\mathrm{~T} / \mathrm{m}$ \\
Quad field gradient (QF2) & 281.9 & $\mathrm{~T} / \mathrm{m}$ \\
Quad field gradient (QD3) & 390.2 & $\mathrm{~T} / \mathrm{m}$ \\
Quad field gradient (QF4) & & \\
& & \\
\hline
\end{tabular}

Table 24: Arc and dispersion suppressor magnet and cell parameters. 


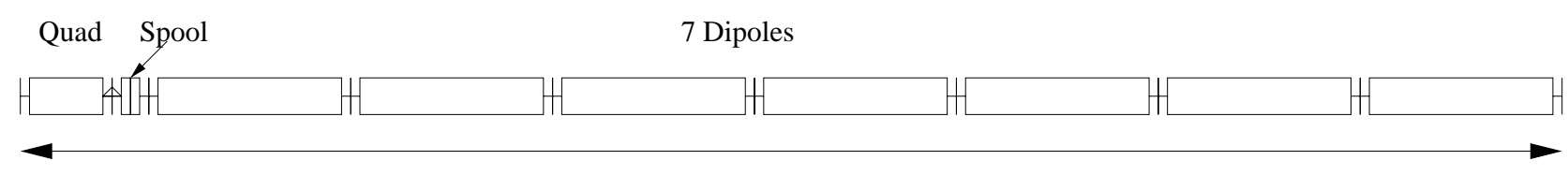

Half cell length $=135.486 \mathrm{~m}$

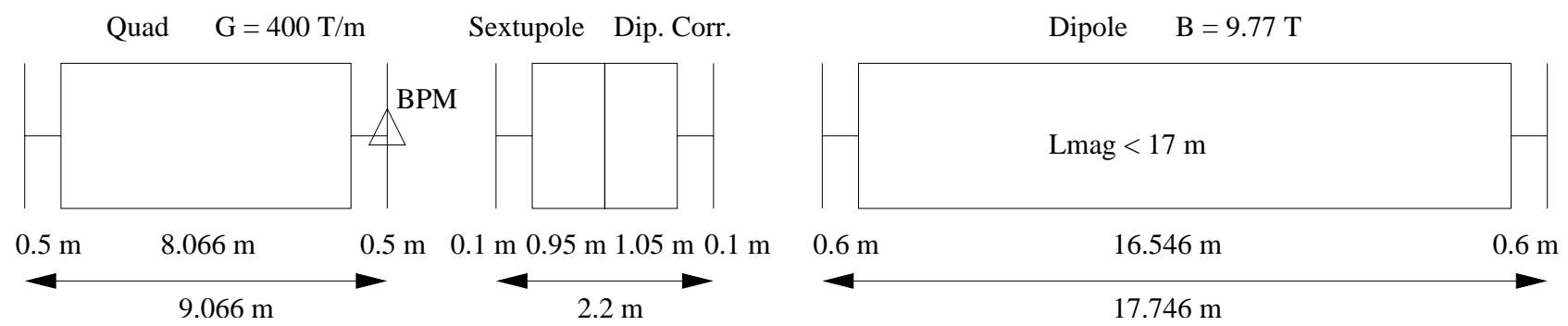

Figure 35: Layout and parameters of a high field arc half cell.

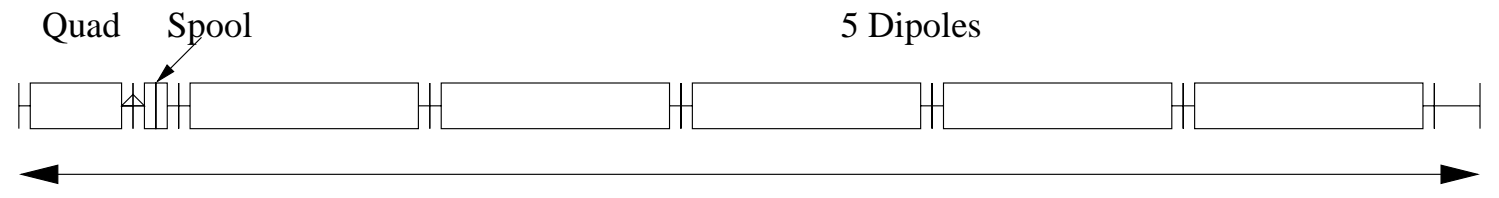

Half cell length $=101.614 \mathrm{~m}$
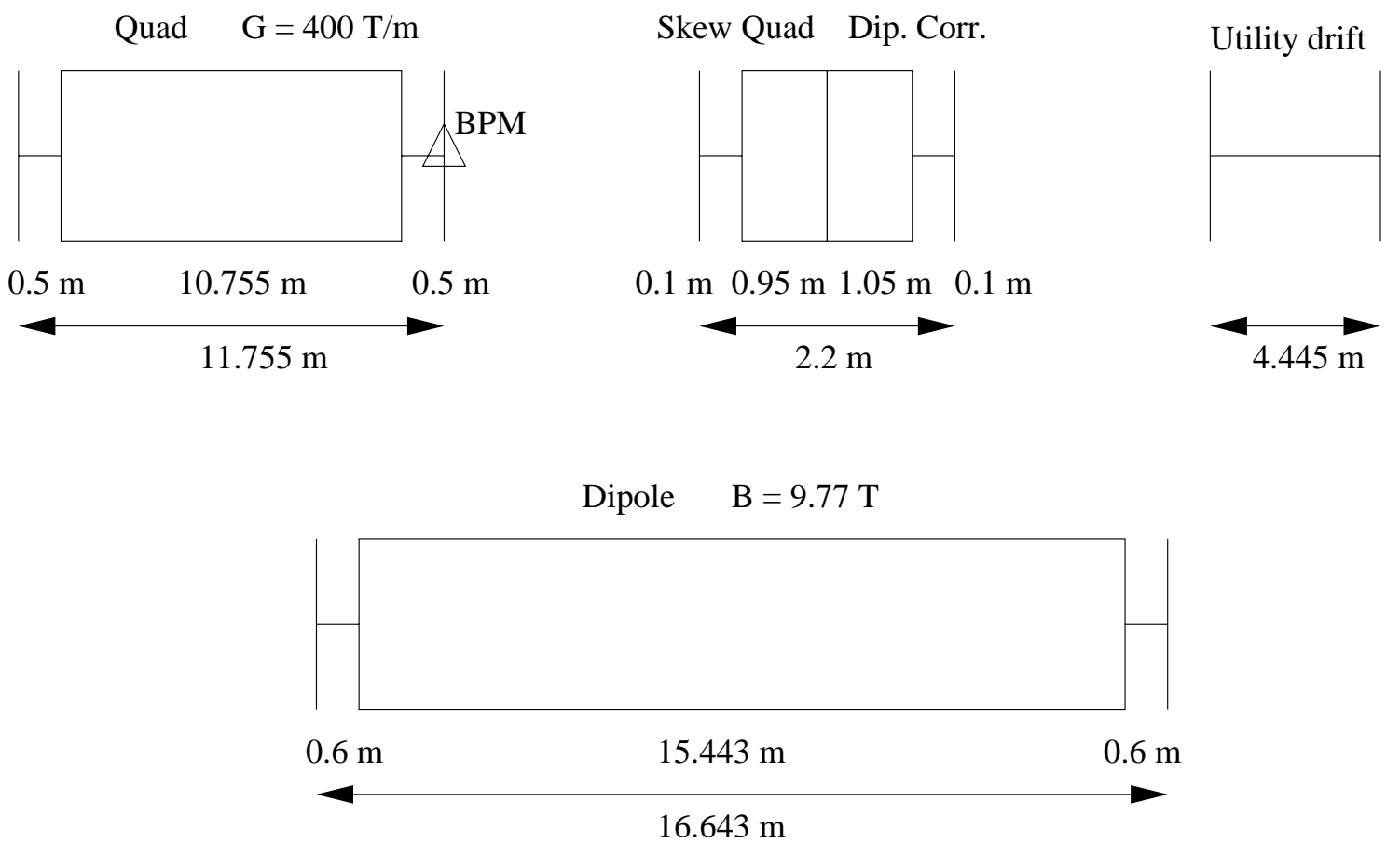

Figure 36: Layout and parameters of a high field dispersion suppressor half cell. 


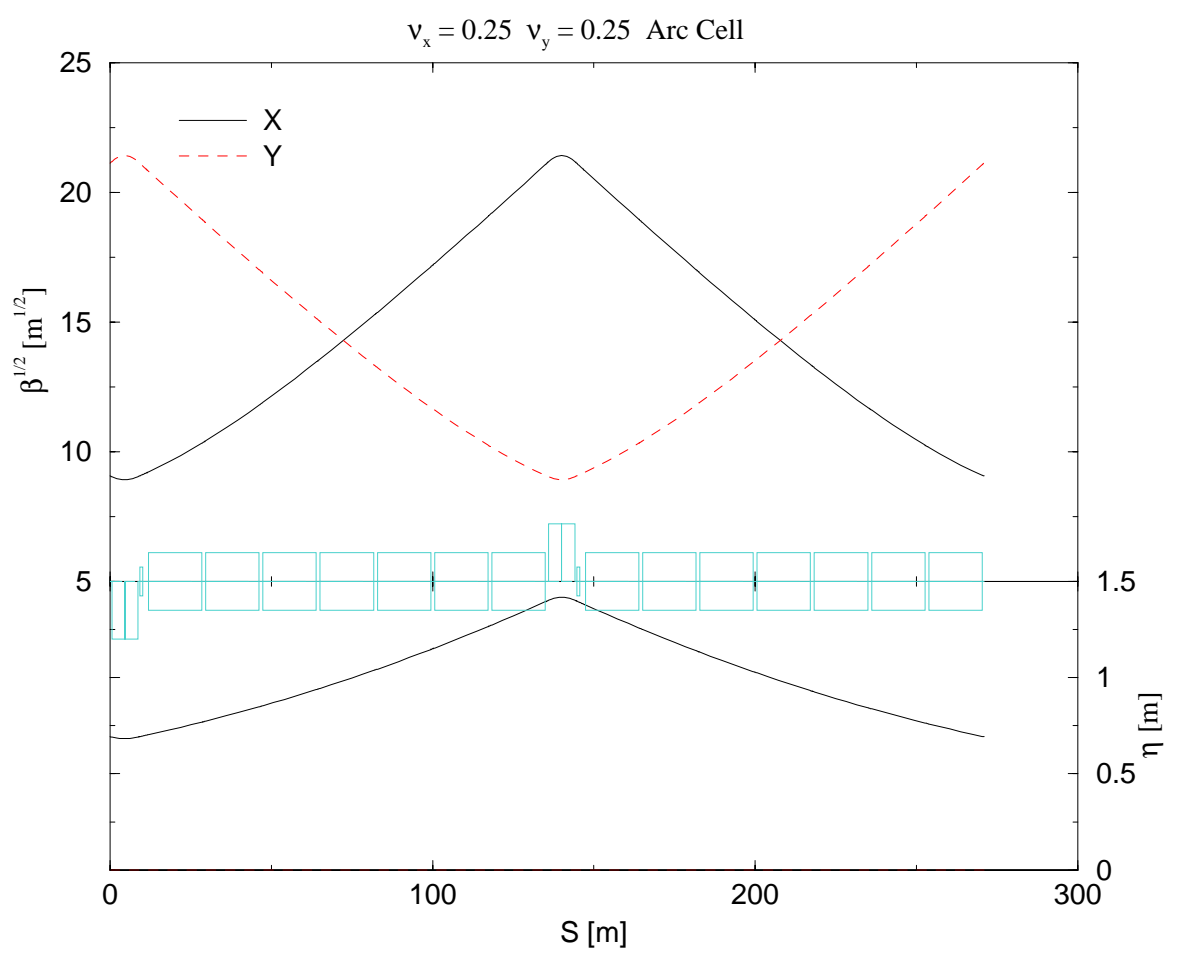

Figure 37: The arc cell Twiss functions. The beam line is shown with the dipoles, spool pieces and the quadrupoles.

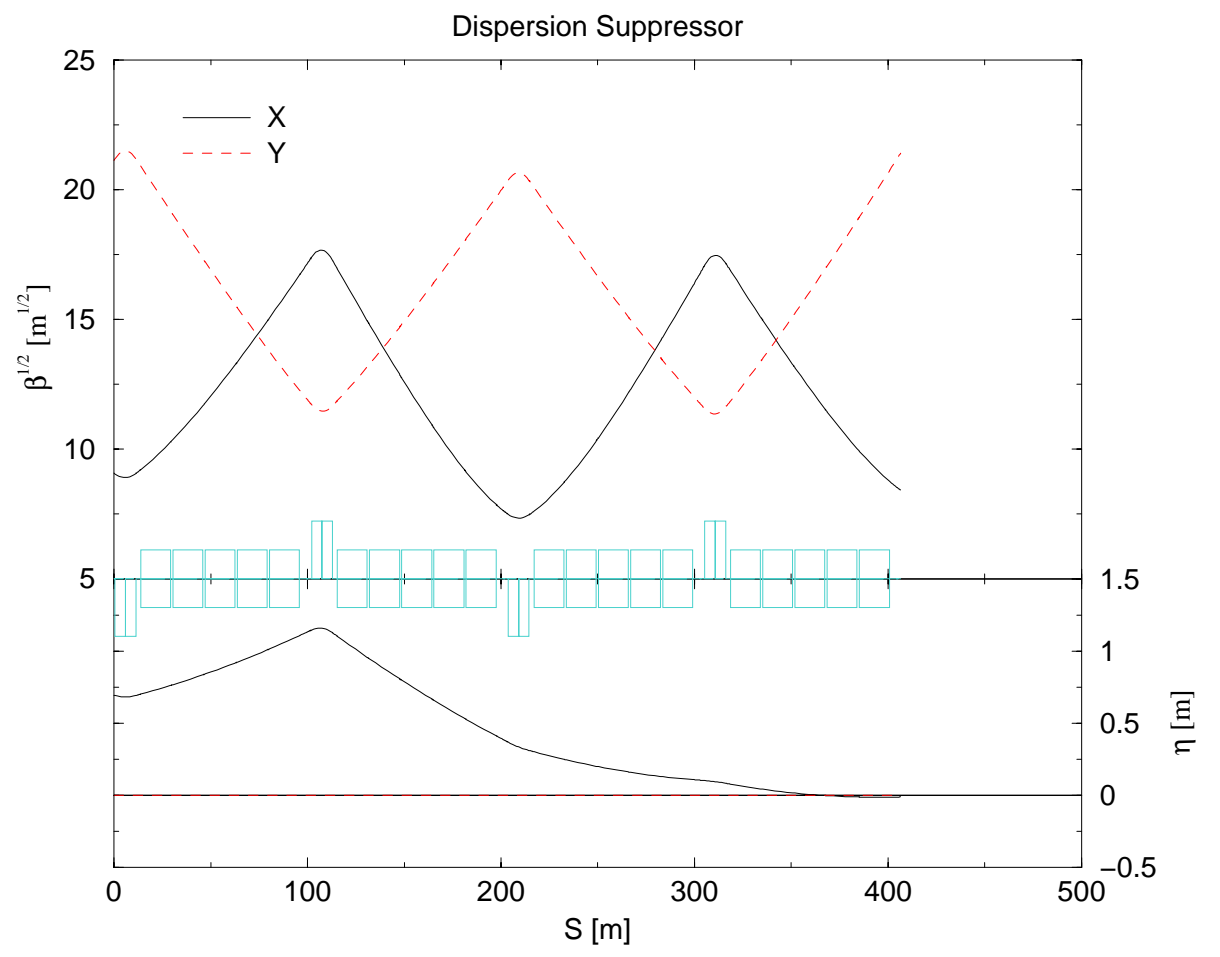

Figure 38: The dispersion suppressor Twiss functions. Each half cell is $3 / 4$ the length of the arc half cell, so the total length is 3 arc half cells long. 


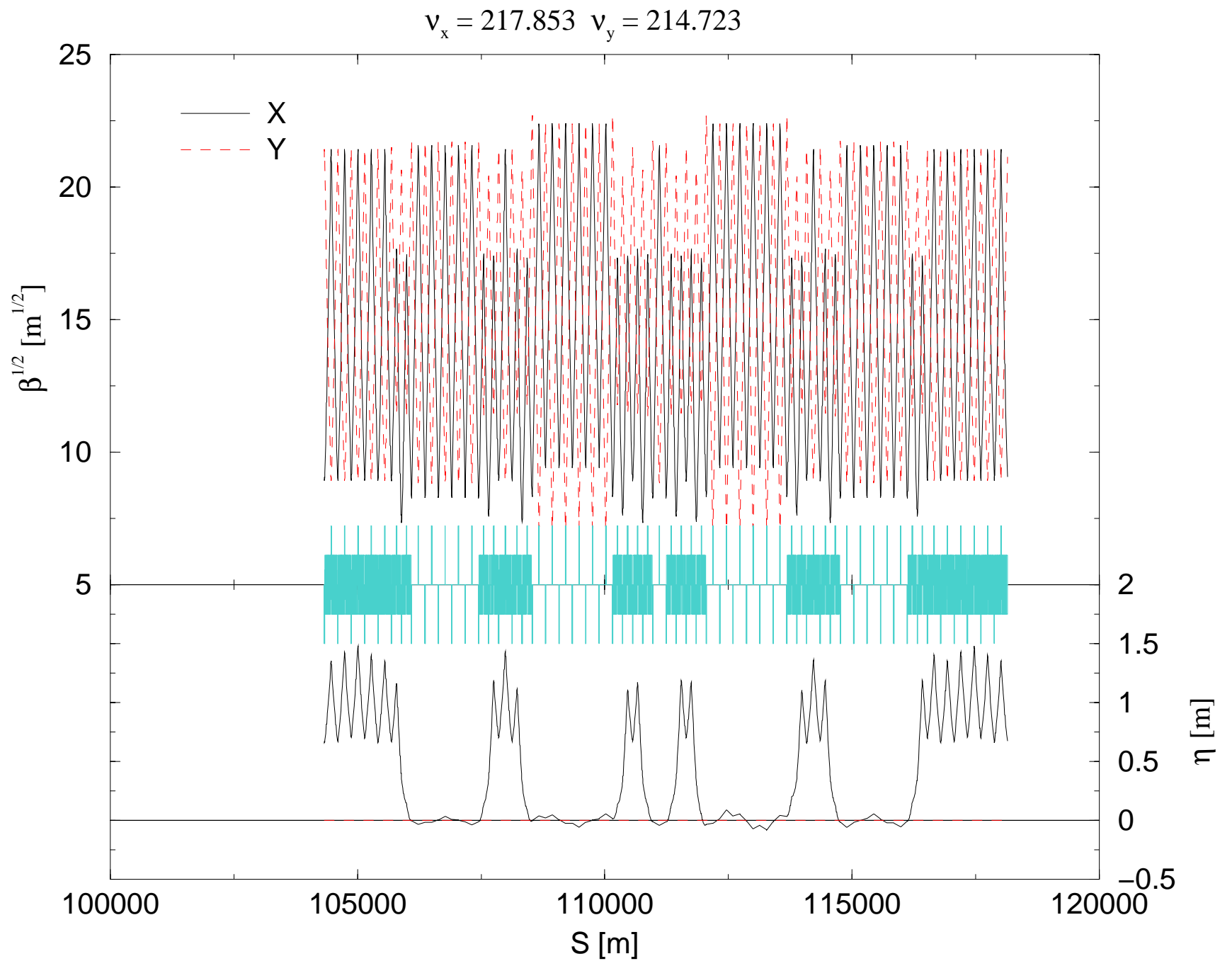

Figure 39: Twiss function in the off-site cluster region on the far side of the ring from the Fermilab site. 


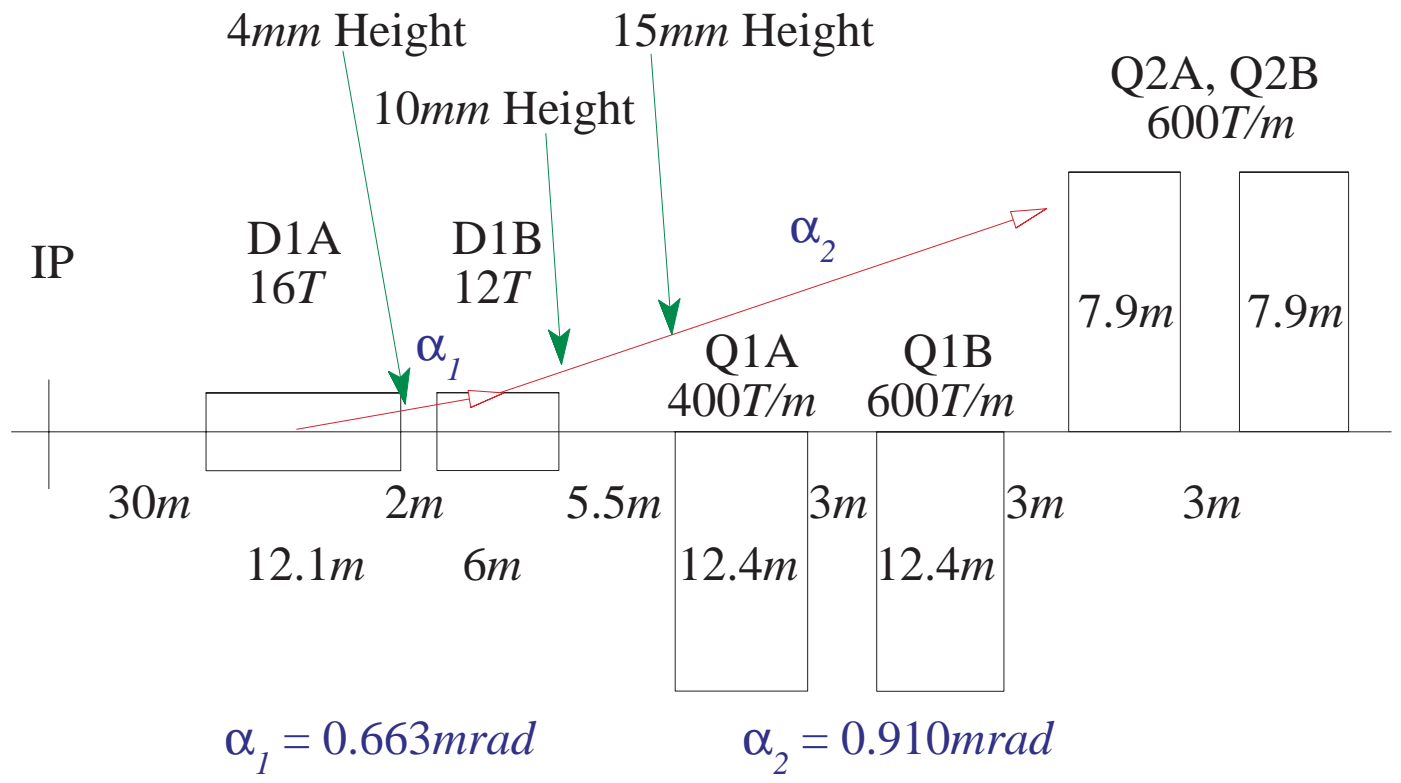

Figure 40: The IR layout. Shown here is the first crossing point dipoles and the doublet quadrupoles Q1 and Q2. Note, the quadrupoles are split into two pieces to provide space for correctors. The beam angles and height relative to the central line are shown. Note, the beam separation is twice the height.

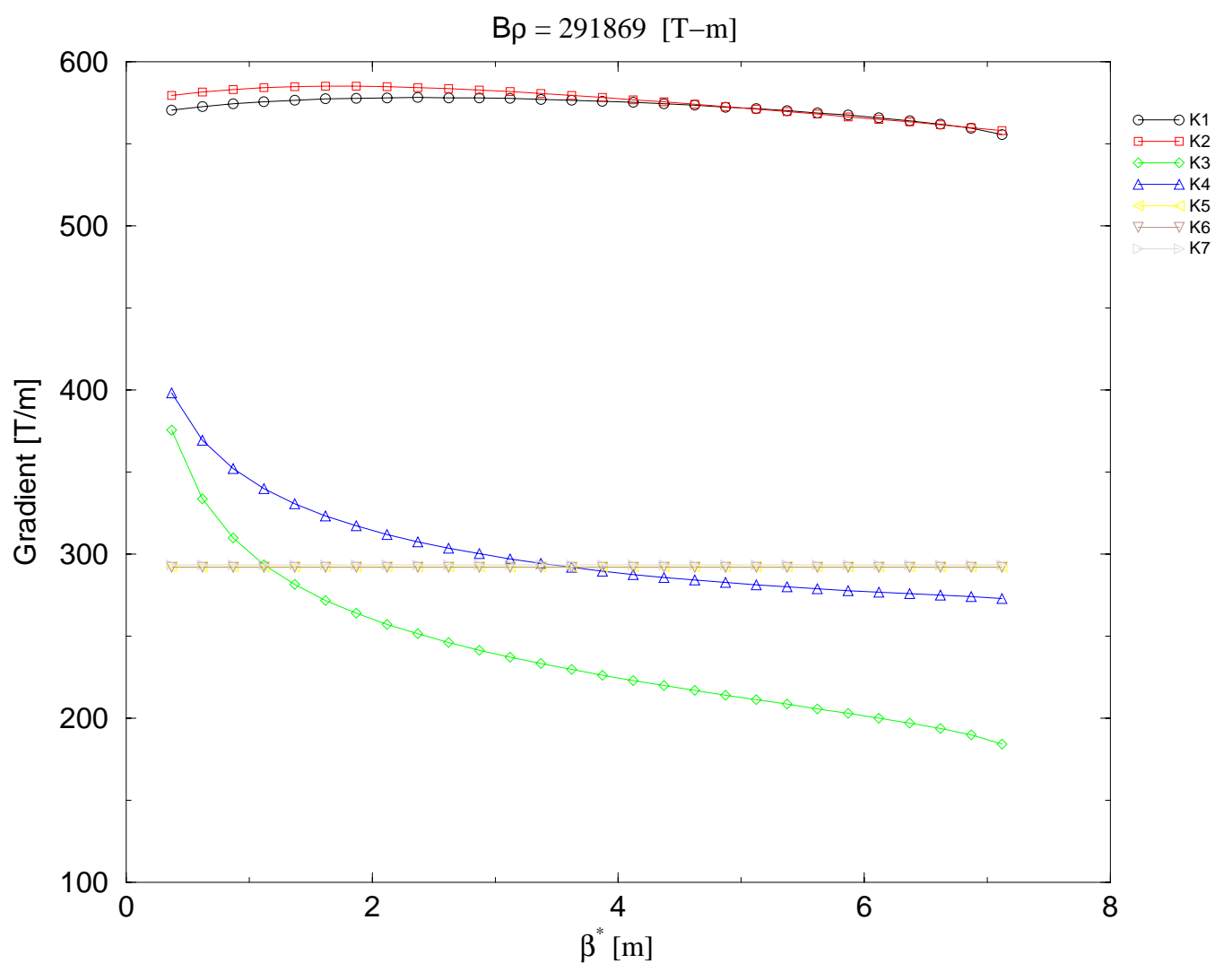

Figure 41: Tuning curves for the interaction region quadrupoles, with flat beam doublet optics. 

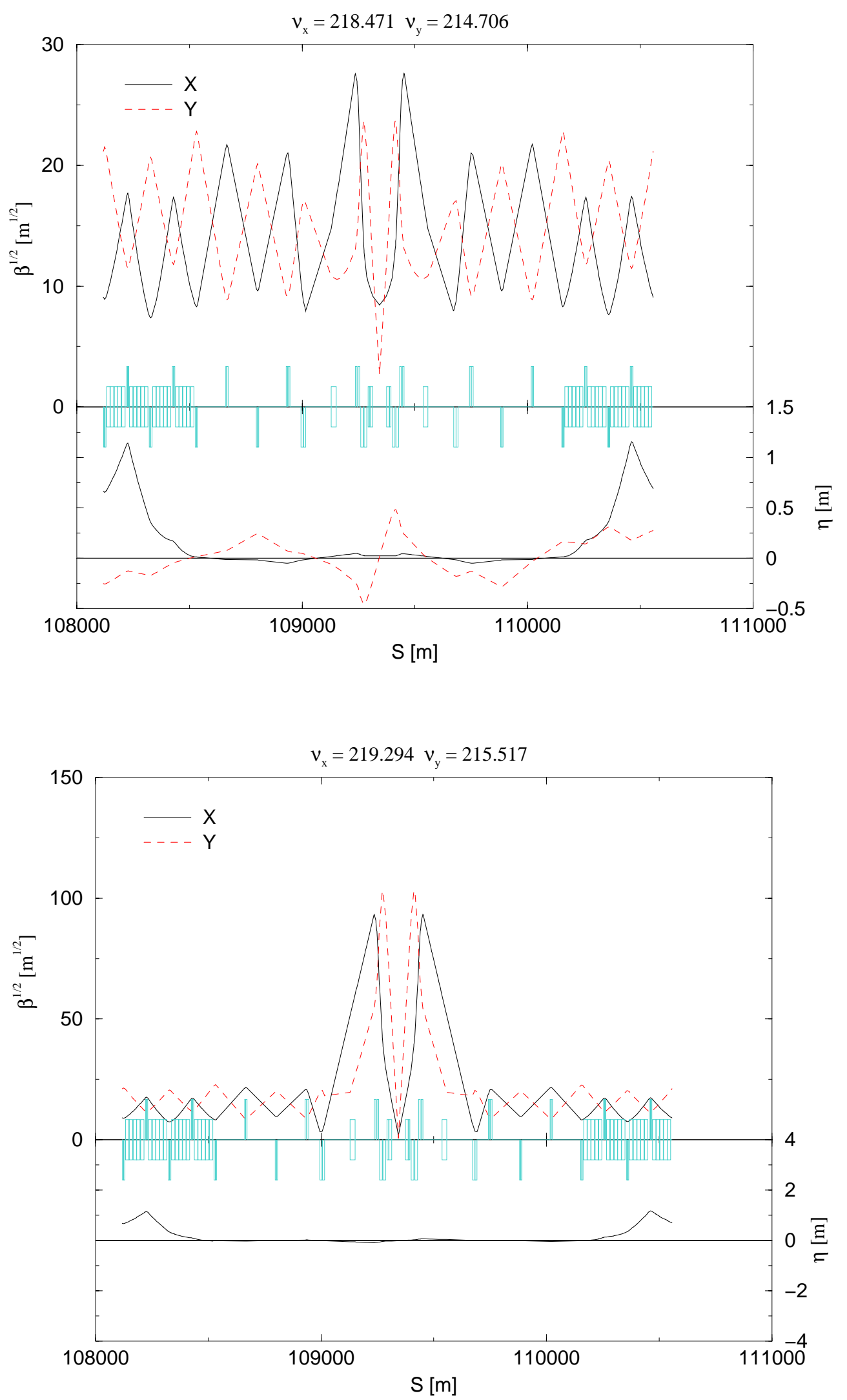

Figure 42: Lattice functions at one of the IPs at injection (top) and in collision (bottom), with flat beam doublet optics. 


\subsubsection{Round Beam Triplet Optics}

Although doublet optics are more naturally suited to the flat beams of the high-field ring, collisions could be created instead using anti-symmetric triplets for the final focusing. The round beam model discussed here is qualitatively similar to the lowfield IR design. The triplet quadrupoles are $400 \mathrm{~T} / \mathrm{m}$ single-bore magnets. Four additional circuits, comprising double-bore $400 \mathrm{~T} / \mathrm{m}$ magnets are also used for optical matching. With a total of 6 independently-tunable quadrupole circuits available it is possible to match the four $\beta$ 's and $\alpha$ 's from the IP into the regular FODO cells, plus hold the phase advance $\Delta \mu$ constant across the IR through the squeeze from $\beta=12.0 \rightarrow 0.50 \mathrm{~m}$. Fixing $\Delta \mu$ eliminates the need for a special phase trombone somewhere in the ring to maintain the nominal operating point. Figure 43 shows the lattice functions through the insertion region at injection and collision. The corresponding magnet gradients are listed in Table 25, and the complete gradient tuning curves through the low-b squeeze appear in Figure 44.

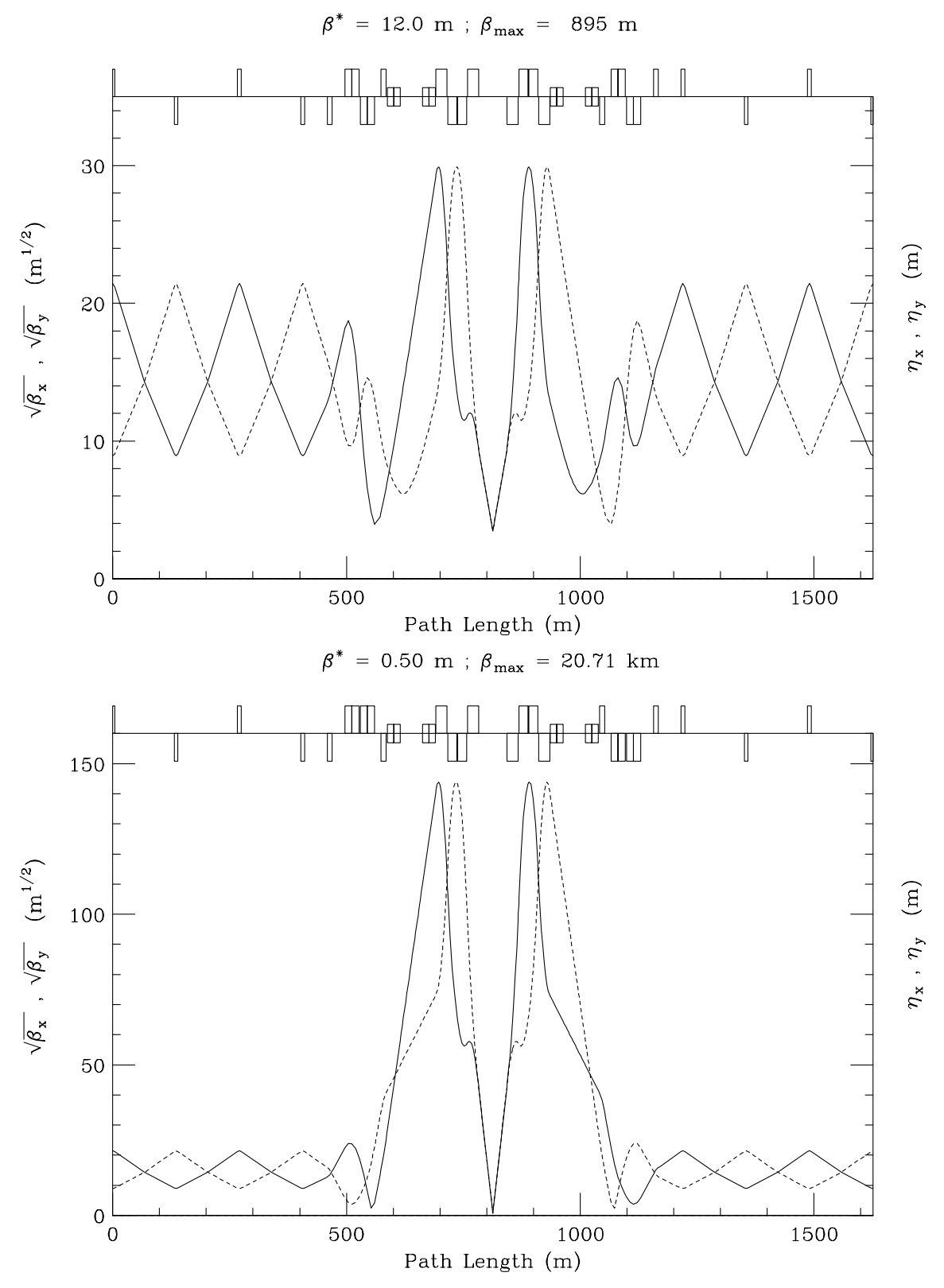

Figure 43: Lattice functions at one of the IPs at injection (top) and in collision (bottom), with round beam triplet optics. 


\begin{tabular}{|cccc|}
\hline Quad \# & $\begin{array}{c}\mathrm{L}_{\text {magnetic }} \\
(\mathrm{m})\end{array}$ & $\begin{array}{c}\text { Gradients }(\mathrm{T} / \mathrm{m}) \\
\beta^{*}=12.00 \mathrm{~m} \\
{\left[\beta_{\max }=895 \mathrm{~m}\right]}\end{array}$ & $\begin{array}{c}\text { Gradients }(\mathrm{T} / \mathrm{m}) \\
\beta^{*}=0.50 \mathrm{~m} \\
{\left[\beta_{\text {max }}=20.7 \mathrm{~km}\right]}\end{array}$ \\
& & & \\
1 & 23.58 & $\mp 400.1$ & $\mp 394.0$ \\
$2 \mathrm{a} \&$ 2b & 18.98 & \pm 397.8 & \pm 382.4 \\
3 & 23.58 & $\mp 400.1$ & $\mp 394.0$ \\
4 & 10.47 & $\mp 229.8$ & \pm 397.9 \\
5a \& 5b & 15.09 & \pm 324.7 & $\mp 391.9$ \\
6a \& 6b & 15.09 & $\mp 342.5$ & $\mp 329.4$ \\
7 & 10.47 & \pm 101.7 & \pm 373.9 \\
\hline
\end{tabular}

Table 25: IR quadrupole gradients at $87.5 \mathrm{TeV} / \mathrm{c}$ for injection $\left(\beta^{*}=12 \mathrm{~m}\right)$ and collision $\left(\beta^{*}=50 \mathrm{~cm}\right)$, with round beam triplet optics. Throughout the squeeze $\Delta \mu_{x}=\Delta \mu_{y}=2.250$ is held fixed. Highlighted entries indicate quadrupoles that change polarity.

The circulating beams are separated vertically everywhere in the ring, except in the triplet quadrupoles. Four $10 \mathrm{~T}$ dipoles between the Q3 and Q4 quadrupoles bring the beams together at the entrance to the triplet for collisions at the IP. Dipoles downstream of the IP separate the beams again vertically and channel them back into the upper and lower rings. A halfcrossing angle of $28.8 \mu$ r gives $10 \sigma$ separation between the beams at the first parasitic crossing $2.823 \mathrm{~m}$ downstream of the IP $\left(\epsilon_{N}=1.5 \mu \mathrm{m}\right.$ at $\left.87.5 \mathrm{TeV} / \mathrm{c}\right)$.

IR Quad Gradient Variations with $\beta^{*}$

$$
\text { [87.5 TeV] }
$$

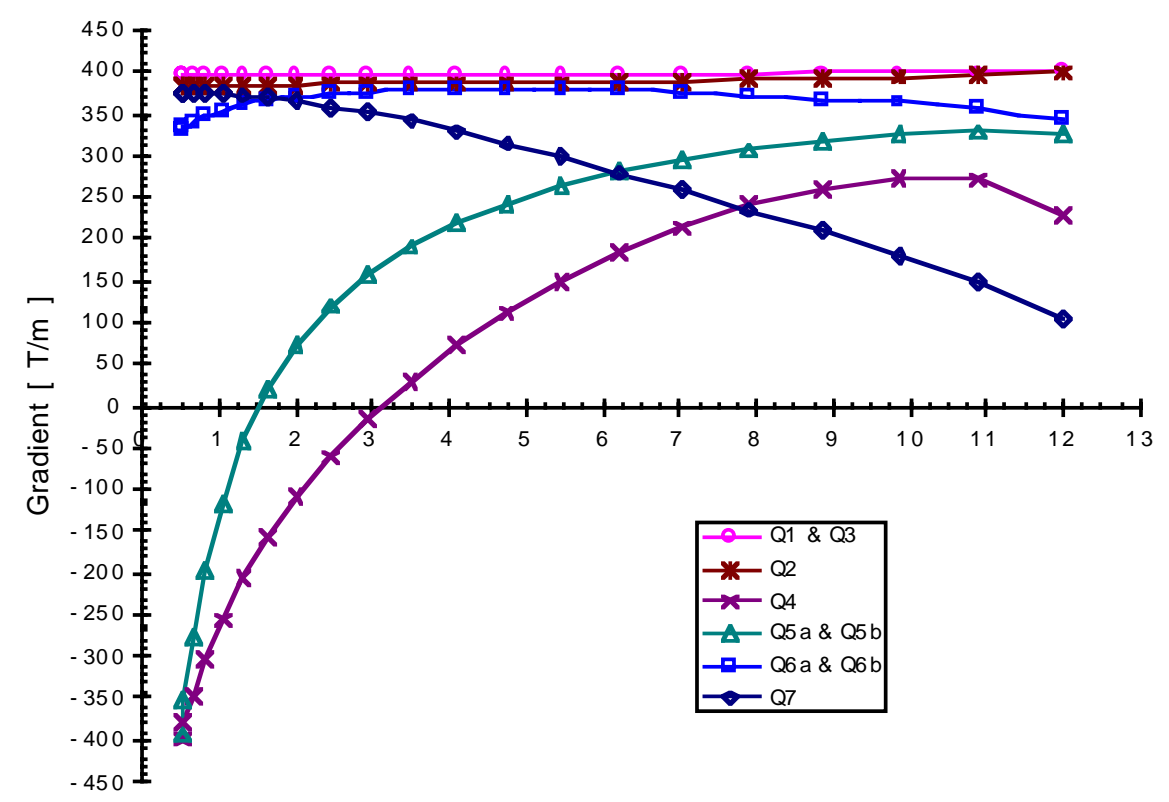

$B^{*}[\mathrm{~m}]$

Figure 44: Tuning curves for the IR quadrupoles, with round beam triplet optics, in a low- $\beta$ squeeze: $\beta^{*}=12.0 \rightarrow 0.50 \mathrm{~m}$. 


\subsection{Interaction Region Magnets}

The flat beam IR and luminosity performance of the high field ring depend significantly on a small number of high field magnets. Given the importance of these few magnets, state of the art superconductors are used in the design. The amount of superconductor used in the magnet will go down and the design will become simpler with the further improvements in superconductor technology.

The parameters of various IR magnets are given in Table 26. The layout is shown in Fig. 40. The major considerations in the design of IR magnets in the high field ring are:

- Small aperture (specially in quadrupoles for generating high gradients)

- Brittle superconductors that must be used for generating high field and/or gradient

- Large Lorentz forces (associated with high fields)

- Small separation between the two apertures (associated with the doublet optics)

The ends of conventional cosine theta designs put a practical limit on the minimum aperture, particularly in quadrupole magnets made with brittle material. To overcome this and other limitations the high field interaction region is based on nontraditional magnet designs with racetrack coils. The design philosophy adopted here is in part similar to the common coil design used in main dipole magnets. These are conductor friendly designs with large bend radii and are suitable for containing large Lorentz forces.

\begin{tabular}{|lccccc|}
\hline Magnet & Field [T] & Gradient [T/m] & Aperture [mm] & Length [m] & Type \\
D1A & 16 & & 25 & 12.1 & 1-in-1 \\
D1B & 12 & & 50 & 6.0 & 1 -in-1 \\
D2 & 12 & & 50 & 11.1 & 2 -in-1 \\
Q1A & & 400 & 30 & 12.4 & 2 -in-1 \\
Q1B & & 600 & 30 & 12.4 & 2-in-1 \\
Q2A & & 600 & 30 & 7.9 & 2-in-1 \\
Q2B & & 600 & 30 & 7.9 & 2-in-1 \\
& & & & & \\
\hline
\end{tabular}

Table 26: Design parameters of the high field interaction region magnets.

The minimum separation between the two apertures in Q1A determines the layout of the entire high field interaction region, and the maximum beam size for the given optics. In addition, it also establishes the maximum pole tip field of this and other magnets. In conventional 2-in-1 designs, the minimum separation is determined by the conductor width required for generating field gradient and the support structure required for containing large Lorentz forces. In the proposed design the amount of conductor between the two apertures is much smaller than on any other side and no support structure is required between the two apertures. This brings a large reduction in spacing (by about a factor of five) between the two apertures. The cross section of the proposed design is shown in Figure 45. In order to facilitate large bend radii, the return path of all turns is further away from the aperture. Field contours and field lines in the aperture of this magnet are also shown in Fig. 45. The design is based on "React and Wind" $\mathrm{Nb}_{3} \mathrm{Sn}$ superconductor with a current density of $2500 \mathrm{~A} / \mathrm{mm}^{2}$ at $12 \mathrm{~T}$.

Magnets based on these design principles use a much larger amount of conductor than that in a conventional design. However, the cost of conductor is not a major issue in designing a few critical high performance magnets. This design also introduces a strong coupling and a cross talk between the two apertures. The super-imposition of a dipole field on the quadrupole coils increases the peak field on the conductor and reduces the maximum achievable gradient. The maximum gradient in Q1A (minimum separation) is, therefore, $400 \mathrm{~T} / \mathrm{m}$ as compared to $600 \mathrm{~T} / \mathrm{m}$ in Q1B, Q2A and Q2B where this effect is much smaller. The goal is to minimize the cross talk induced harmonics with the exception of the dipole field. By symmetry, the normal even harmonics and skew odd harmonics are theoretically zero. The target field harmonics are given in Table 62, which includes harmonics from both design and construction errors. These values will be obtained either by design or by correction. Table 62 harmonics are dominated by design errors, which are expected to decrease as the design evolves. All four quadrupoles will have a different cross section. 


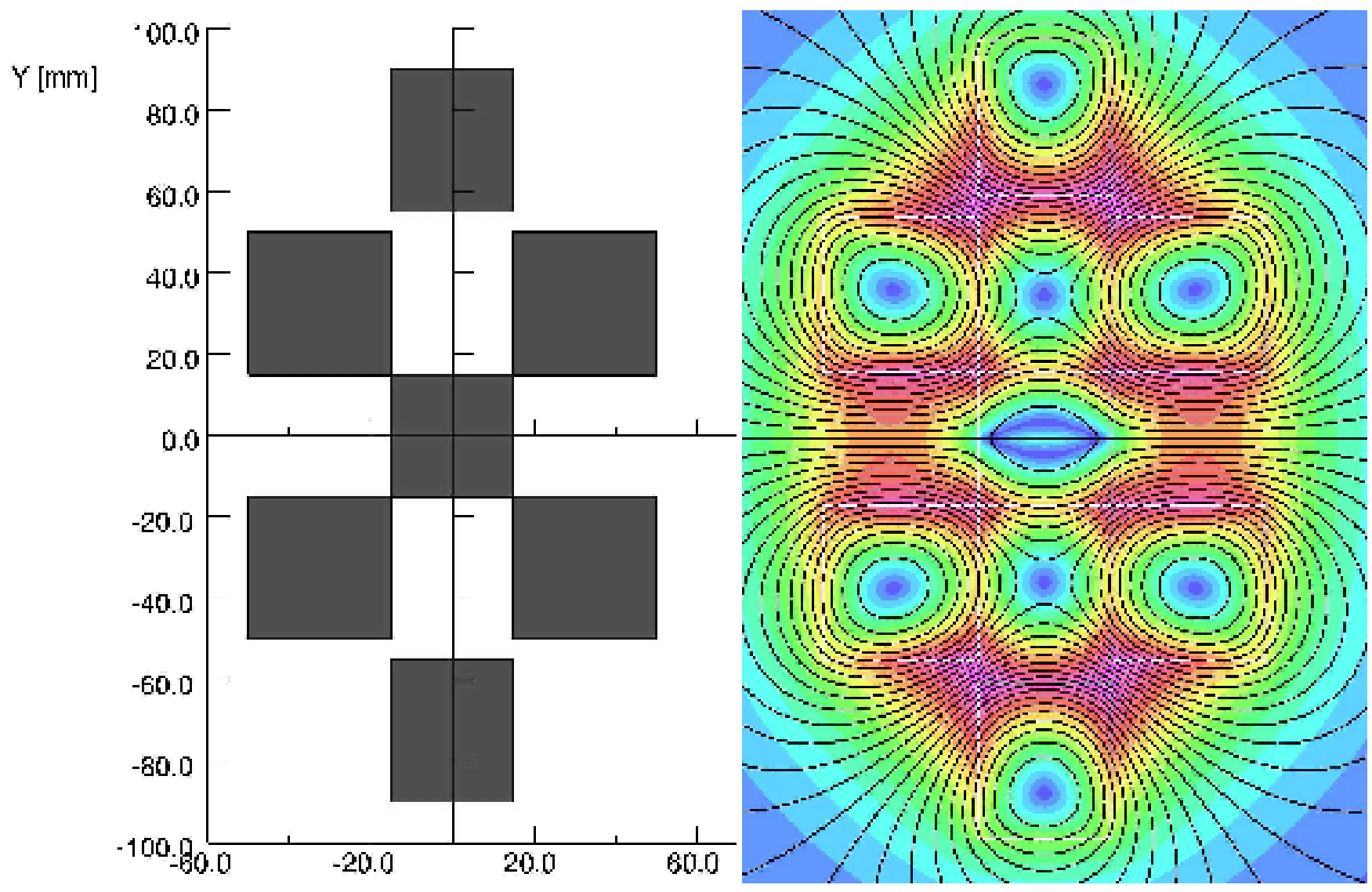

Figure 45: A conceptual design of 2-in-1 IR Quadrupole Q1A (left) and field contours and field lines in the aperture region of the magnet (right). The design minimizes the spacing between two apertures.

The high field interaction region uses three types of dipole magnets. The design of all insertion region dipoles is also based on racetrack coils. D1A, D1B (one each on each side) are single aperture dipoles and D2 (two on each side) is a 2-in-1 dipole. The operating field in the smaller aperture $(25 \mathrm{~mm})$ dipole D1A is $16 \mathrm{~T}$, with a quench field of $\sim 18 \mathrm{~T}$. It uses high temperature superconductor (BSCCO 2212) in a hybrid design. The operating field in the larger aperture (50 mm) dipole D1B is reduced to $12 \mathrm{~T}$ to reduce the Lorentz forces in larger aperture. This field can be entirely obtained by $\mathrm{Nb}_{3} \mathrm{Sn}$ superconductor.

The interaction region will also contain a number of corrector magnets. The higher order harmonic correctors will be based on multi-layer coils within the same cold mass. 


\subsection{Dynamic Aperture and Field Quality}

To estimate the effect of the magnetic field errors on the single particle dynamic, the nonlinear chromaticity, the tune spread from nonlinear fields and the 1000-turn dynamic aperture were computed at injection and storage energy. 1000 turns is equivalent to 0.7 seconds real time in the VLHC.

The tune change as a function of relative momentum deviation is computed for particles of up to three times the rms momentum deviation. The tune spread from nonlinear fields is computed for on-momentum particles with betatron amplitudes of up to $6 \sigma$ of the transverse rms beam size. Five different ratios of horizontal to vertical emittance were chosen.

To determine the dynamic aperture, particles were tracked over 1000 turns ( 0.7 seconds real time). 10 different random distributions ("seeds") of magnetic field errors were investigated. The total transverse emittance is distributed onto the horizontal and vertical emittance in 5 ratios:

$$
\begin{aligned}
& \epsilon_{x}=0.96 \cdot \epsilon_{\text {total }} \quad \epsilon_{y}=0.04 \cdot \epsilon_{\text {total }} \\
& \epsilon_{x}=0.75 \cdot \epsilon_{\text {total }} \quad \epsilon_{y}=0.25 \cdot \epsilon_{\text {total }} \\
& \epsilon_{x}=0.50 \cdot \epsilon_{\text {total }} \quad \epsilon_{y}=0.50 \cdot \epsilon_{\text {total }} \\
& \epsilon_{x}=0.25 \cdot \epsilon_{\text {total }} \quad \epsilon_{y}=0.75 \cdot \epsilon_{\text {total }} \\
& \epsilon_{x}=0.04 \cdot \epsilon_{\text {total }} \quad \epsilon_{y}=0.96 \cdot \epsilon_{\text {total }}
\end{aligned}
$$

For both, injection and storage energy, a round beam was assumed with equal horizontal and vertical emittance. The accommodation of a round beam is more demanding than the flat beam at storage that results from radiation damping. No synchrotron motion was included but particles had a relative momentum deviation of three times the rms momentum deviation.

Experiments have shown that the dynamic aperture can be computed for storage rings with nonlinear field errors within a $30 \%$ error when the field errors are well known $[46,47]$. For a future machine, however, a larger safety margin is required.

\subsubsection{Injection}

The effect of the magnetic field quality on the dynamic aperture was investigated at the injection energy of $10 \mathrm{TeV}$. At injection the beam size has its maximum and errors in the arc magnets dominate the dynamic aperture. With the current one-turn injection scenario, a storage time in the order of seconds is sufficient. In the lattice that is used for the evaluation, no interaction regions were inserted since the dynamic aperture is dominated by the arcs. Arc magnet error tables version 1.0 were used (see Tabs. 58 and 59). In these tables, field errors at injection were estimated for a $2.0 \mathrm{~T}$ field in the dipoles. However, an injection of a $10 \mathrm{TeV}$ beam would correspond to a $1.4 \mathrm{~T}$ field in the dipoles. The difference in the main field should not affect field errors cause by geometry. Only the sextupole component in the dipoles, caused by coil magnetization, is expected to be materially different. The systematic error were computed as the maximum possible absolute value with given mean and uncertainties. Random errors were created from a Gaussian distribution, cut at two sigma.

Quadrupoles were horizontally and vertically displaced randomly with a Gaussian distribution with a $0.3 \mathrm{~mm}$ rms value, cut at three sigma. In addition, beam position monitors were displaced with a $0.2 \mathrm{~mm}$ rms value. The resulting non-zero closed orbit was corrected to zero in the beam position monitors with horizontal orbit correctors at focusing quadrupoles and vertical orbit correctors at defocusing quadrupoles. Skew quadrupole errors in the arcs were disregard and no coupling correction was necessary. The transverse tunes were set to $(218.190,212.180)$. Both horizontal and vertical chromaticity were set to 2 . Tracked particles had a momentum deviation of up to $\Delta p / p=7.2 \cdot 10^{-4}$, corresponding to three times the rms of the momentum distribution. The physical aperture of $1 \mathrm{~cm}$ in the arcs corresponds to $35 \sigma$ of the transverse beam size in the quadrupoles.

In Fig. 46 the horizontal and vertical tunes are shown as a function of the relative momentum deviation. With relative momentum deviations of up to 0.00072 , the tunes shift by up to 0.008 . Fig. 47 shows the tune space needed for on-momentum particles with betatron amplitudes of up to 6 sigma of the transverse rms beam size. The size of the needed tune space is dominated by the systematic octupole error in the dipole. This is shown in Fig. 48 where the systematic octupole errors were set to zero. Fig. 49 shows dynamic aperture averaged over the 10 seeds along with the minimum of the 10 seeds. The error bars of the average dynamic aperture are the rms value of 10 seeds. Although linear coupling and synchrotron motion were disregarded in the determination of the dynamic aperture, the field quality with the magnet field error table V1.0 is sufficient for injection. In addition, A reduction in the systematic octupole error is likely to yield a further gain in dynamic aperture since this field error dominates the tune spread from nonlinear magnetic field errors. 


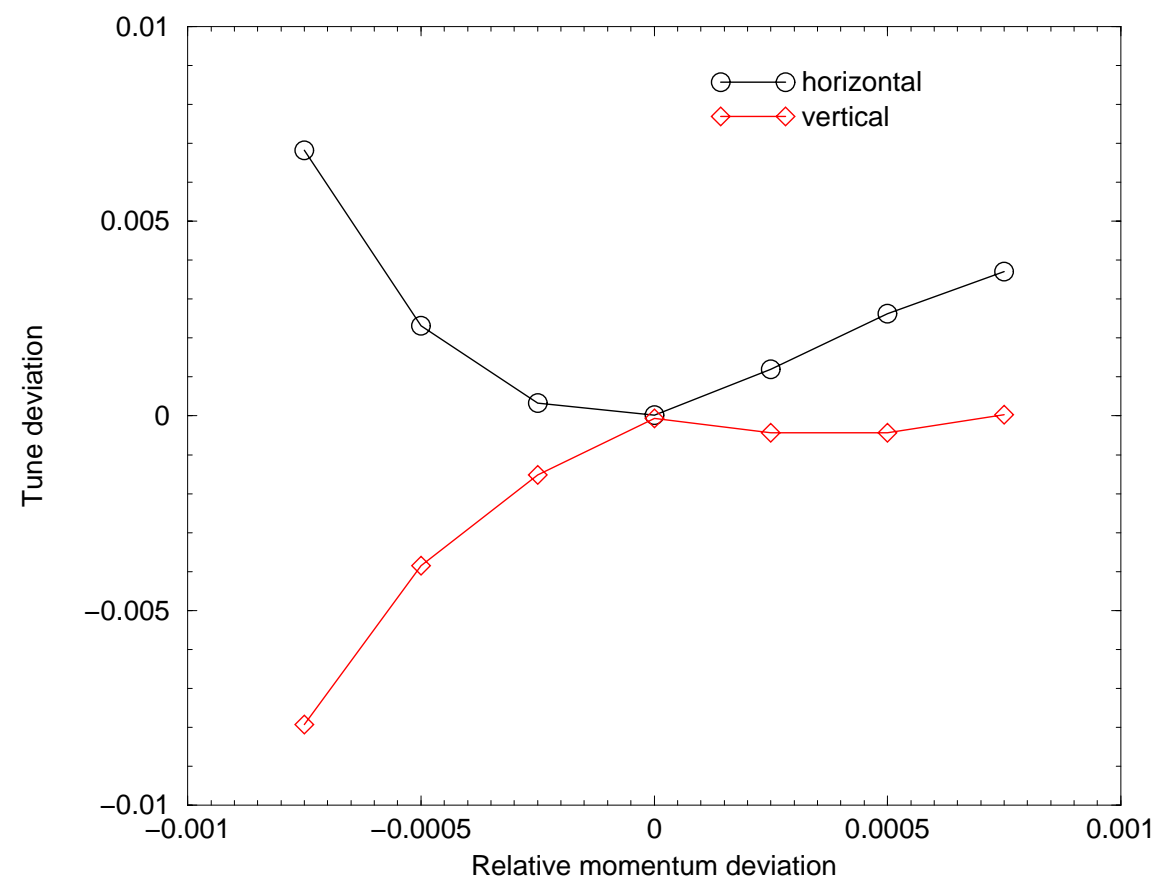

Figure 46: Horizontal and vertical tune as a function of the relative momentum deviation at an injection energy of 10 TeV. The horizontal scale corresponds to approximately $3 \sigma$ of the momentum distribution.

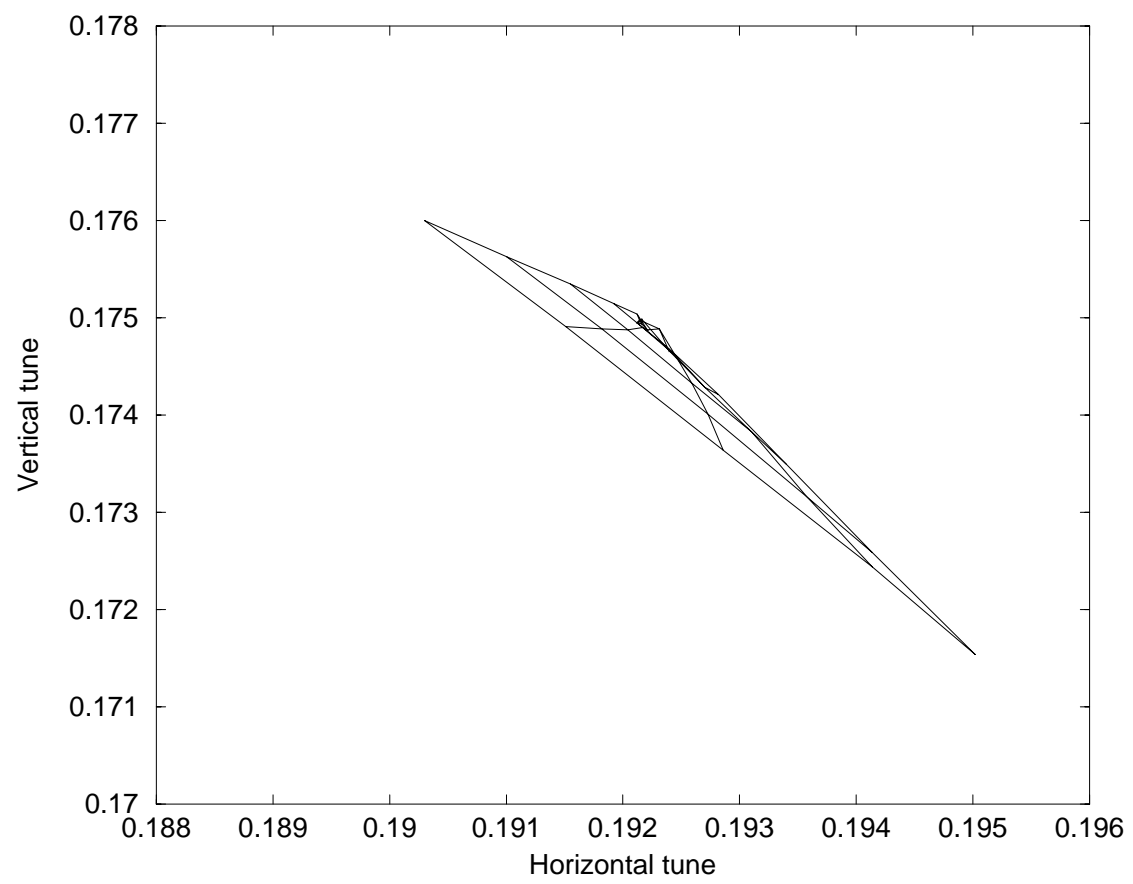

Figure 47: Tune space needed for on-momentum particles with betatron amplitudes of up to 6 sigma of the transverse rms beam size, at an injection energy of $10 \mathrm{TeV}$. 


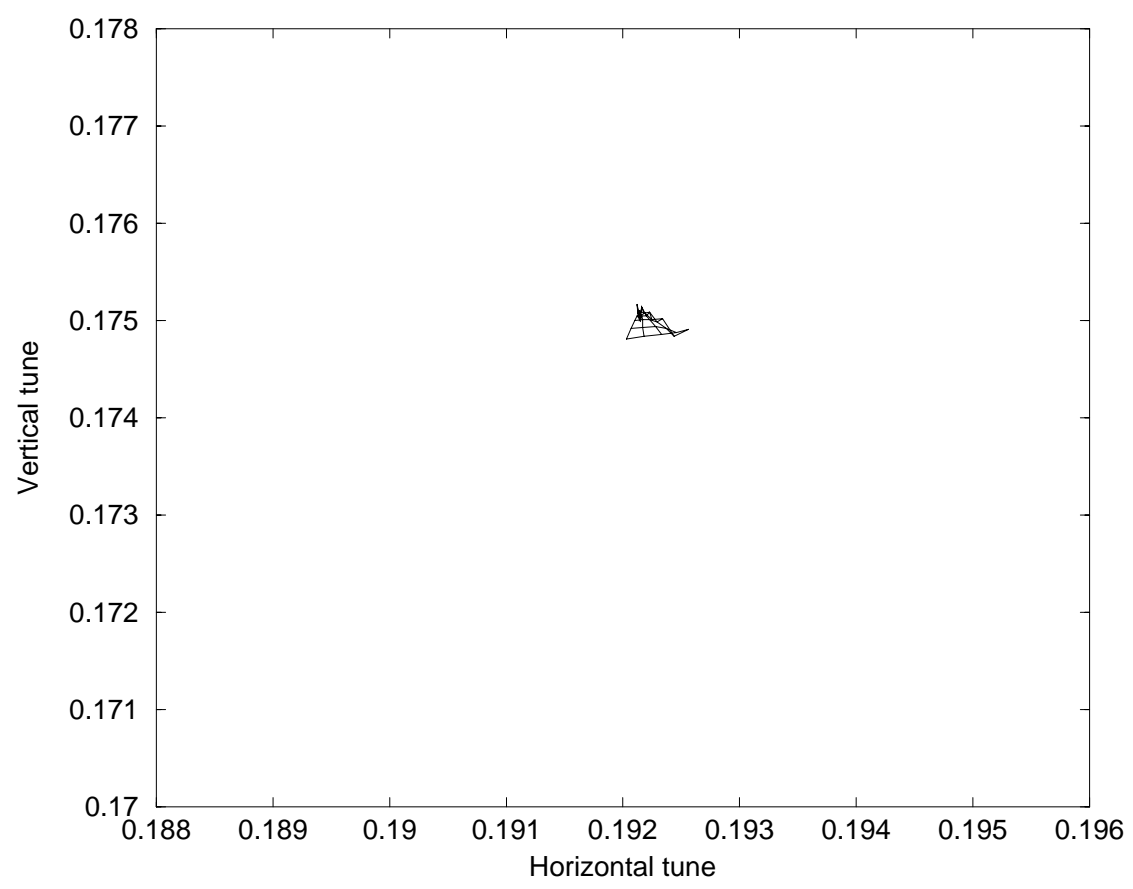

Figure 48: Tune space needed for on-momentum particles with betatron amplitudes of up to 6 sigma of the transverse rms beam size, without systematic octupole errors in dipoles, at an injection energy of $10 \mathrm{TeV}$.

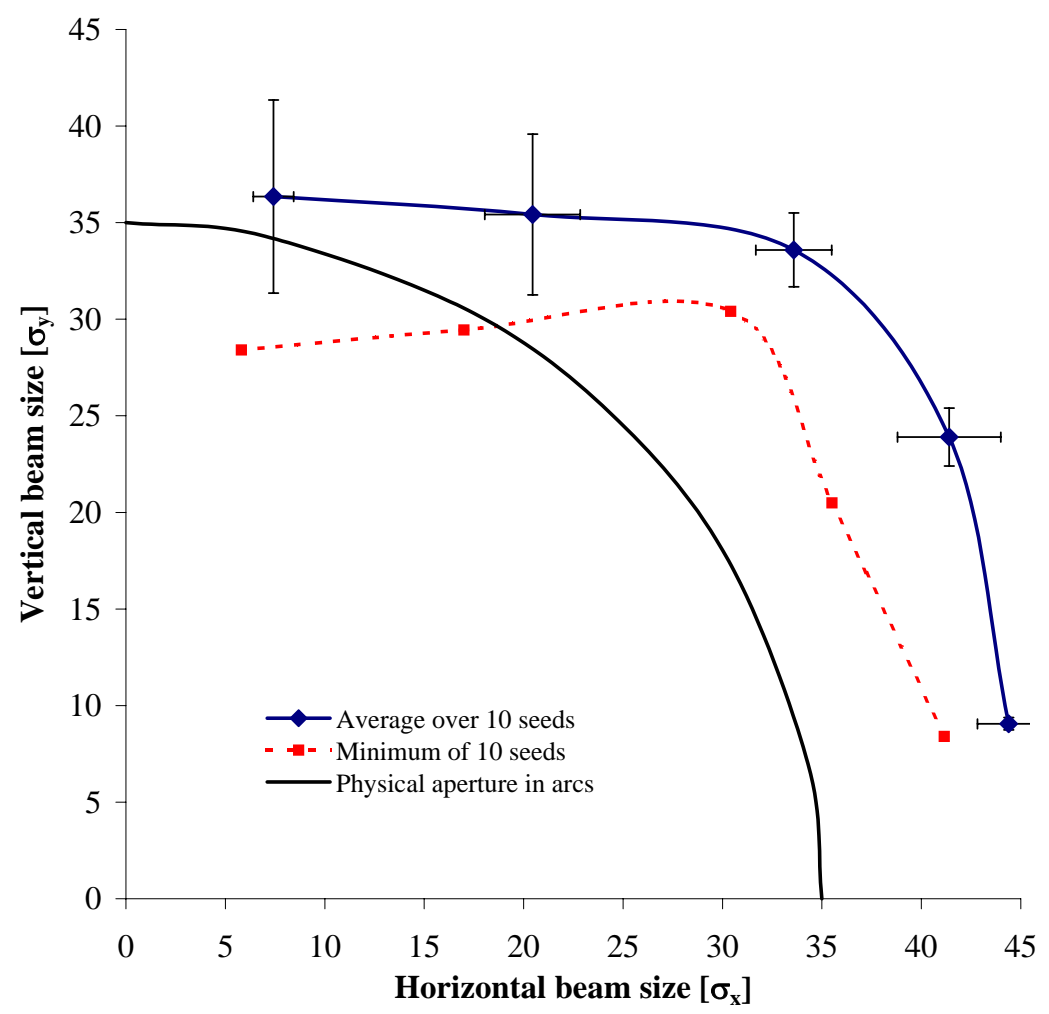

Figure 49: 1000-turn dynamic aperture at an injection energy of $10 \mathrm{TeV}$. Shown are the average over 10 seeds, the worst of 10 seeds, and the physical aperture, in units of the transverse rms beam size. The error bars of the average dynamic aperture are the rms value of 10 seeds. 


\subsubsection{Minimum Injection Energy}

For a given maximum energy, how low can the injection energy be? An estimate of the plausible maximum "dynamic energy range" of a high field ring is crucial in comparing staged and non-staged VLHC scenarios. The tracking results above show that the injection energy can readily be reduced below $10 \mathrm{TeV}$ - that the nominal dynamic energy range of about 10 is conservative. Although an exact discussion of the complex issues which determine the maximum range is not possible here, nonetheless some preliminary observations can be made.

HERA shows that a dynamic energy range of 20 works, despite strong persistent current and snap-back effects at injection and at the start of the acceleration ramp. These effects will be stronger at the LHC, which has a dynamic range of about 16. A "rule of thumb" physical model rests in the hysteretic behavior of $\left\langle b_{2}\right\rangle$ - the systematic sextupole in the arc dipoles - as sketched in Figure 50. The value of $\left\langle b_{2}\right\rangle$ has a minimum value on the up-going side of the hysteresis curve. Both HERA and LHC inject close to this minimum. It is practically impossible to operate at dipole currents much below this minimum, because of the rapid rate of change of $\left\langle b_{2}\right\rangle$ with current, and because of the even more severe hysteresis effects. The minimum field value $B_{\min }$ is expected to be about $0.5 \mathrm{~T}$ for the $\mathrm{Nb}_{3} \mathrm{Sn} 10 \mathrm{~T}$ high field dipole. Hence, a dynamic energy range of about 20 should be possible for the high field ring, corresponding to a minimum injection energy of about $5 \mathrm{TeV}$.

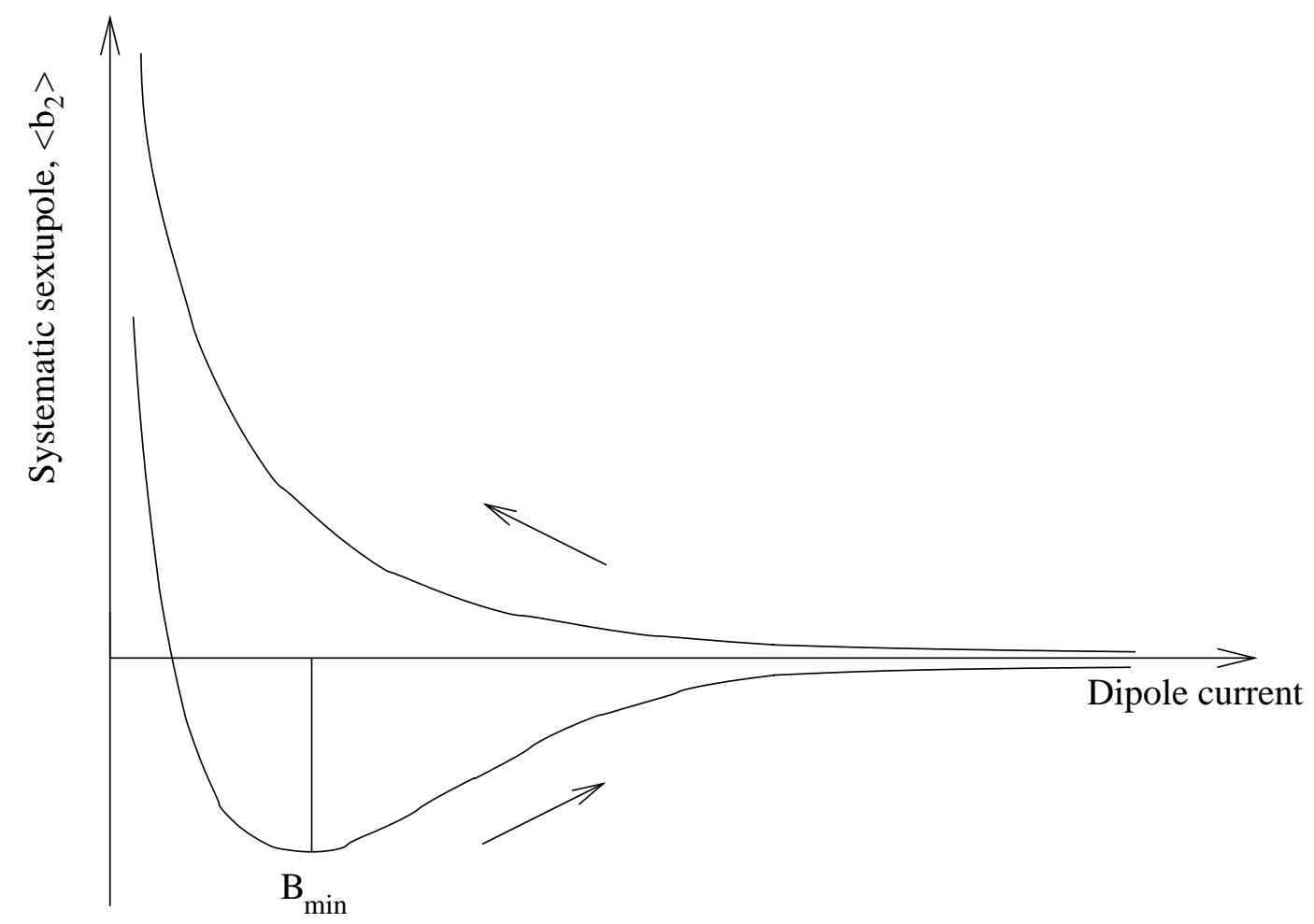

Figure 50: Sketch of systematic sextupole hysteresis in superconducting arc dipoles. It is practically impossible to operate at dipole currents below the minimum value of $\left\langle b_{2}\right\rangle$, which is expected to occur at $B_{\min } \approx 0.5 \mathrm{~T}$ in a $10 \mathrm{~T}$ dipole.

Other considerations also come into play. As already discussed, the half cell length is the same in both low and high field rings. This leads to tighter focusing than might otherwise be chosen for the high field ring. Thanks to adiabatic damping, the injected beam in the high field ring is therefore much smaller than the beam at injection in the low field ring. Consequently, the high field ring is relatively robust against systematic errors such as $\left\langle b_{2}\right\rangle$, as shown in Equation 5 and Figure 7.

At HERA and the LHC the sextupole components are measured in a small number of reference magnets up the ramp, and corrections are applied. In addition, the LHC hopes to measure and correct the chromaticity at about a $1 \mathrm{~Hz}$ rate up the acceleration ramp. A reliable and continuous beam based chromaticity measurement and correction system is required to achieve the largest dynamic energy range, in the presence of strong persistent current effects.

Recent progress in magnet development may lead to significant suppression of the snap-back effect [45]. If snap-back dynamics can be sufficiently suppressed, then closer attention can be paid to the passive correction of injection field harmonics through conventional means (such as shims), and dynamic energy ranges larger than 20 might be possible. 


\subsubsection{Storage}

At the storage energy of $87.5 \mathrm{TeV}$ the beam size is small compared the physical aperture. In the arcs the physical aperture is larger than $100 \mathrm{rms}$ beam sizes. The dynamic aperture is dominated by magnetic field errors in the interaction region magnets where the beta functions reach values of several kilometers. Storage times are several hours. Here the case of round beams at the end of acceleration is considered.

Version 1.1 of the arc magnet errors and version 1.0 of the interaction region magnet errors were used (see Tabs. 60, 61, and 62). However, only Q1 and Q2 magnets in the interaction regions were assigned errors since no estimates exist for the other magnets. Tracking was done without quadrupole displacements. If such displacements are introduced and corrected in the arcs, the small residual orbit distortions leaking into the interaction regions make any test particle unstable. As for injection, the transverse tunes were set to $(218.190,212.180)$. Tracked particles had a momentum deviation of up to $\Delta p / p=1.9 \cdot 10^{-4}$, corresponding to three times the rms of the momentum distribution.

In Fig. 51 the horizontal and vertical tunes are shown as a function of the relative momentum deviation. With relative momentum deviations of up to 0.0002 , the tunes shift by up to 0.005 . Fig. 52 shows the tune space needed for on-momentum particles with betatron amplitudes of up to 6 sigma of the transverse rms beam size. Only a few $10^{-3}$ are needed in tune space. Fig. 53 shows dynamic aperture averaged over the 10 seeds along with the minimum of the 10 seeds. The dynamic aperture is about $35 \sigma$ of the transverse rms beam size. Although this appears to be a large aperture, not all interaction region magnets had errors assigned. Furthermore, the closed orbit error appears to have a significant effect on particle stability. The closed orbit error and its correction warrant careful study.

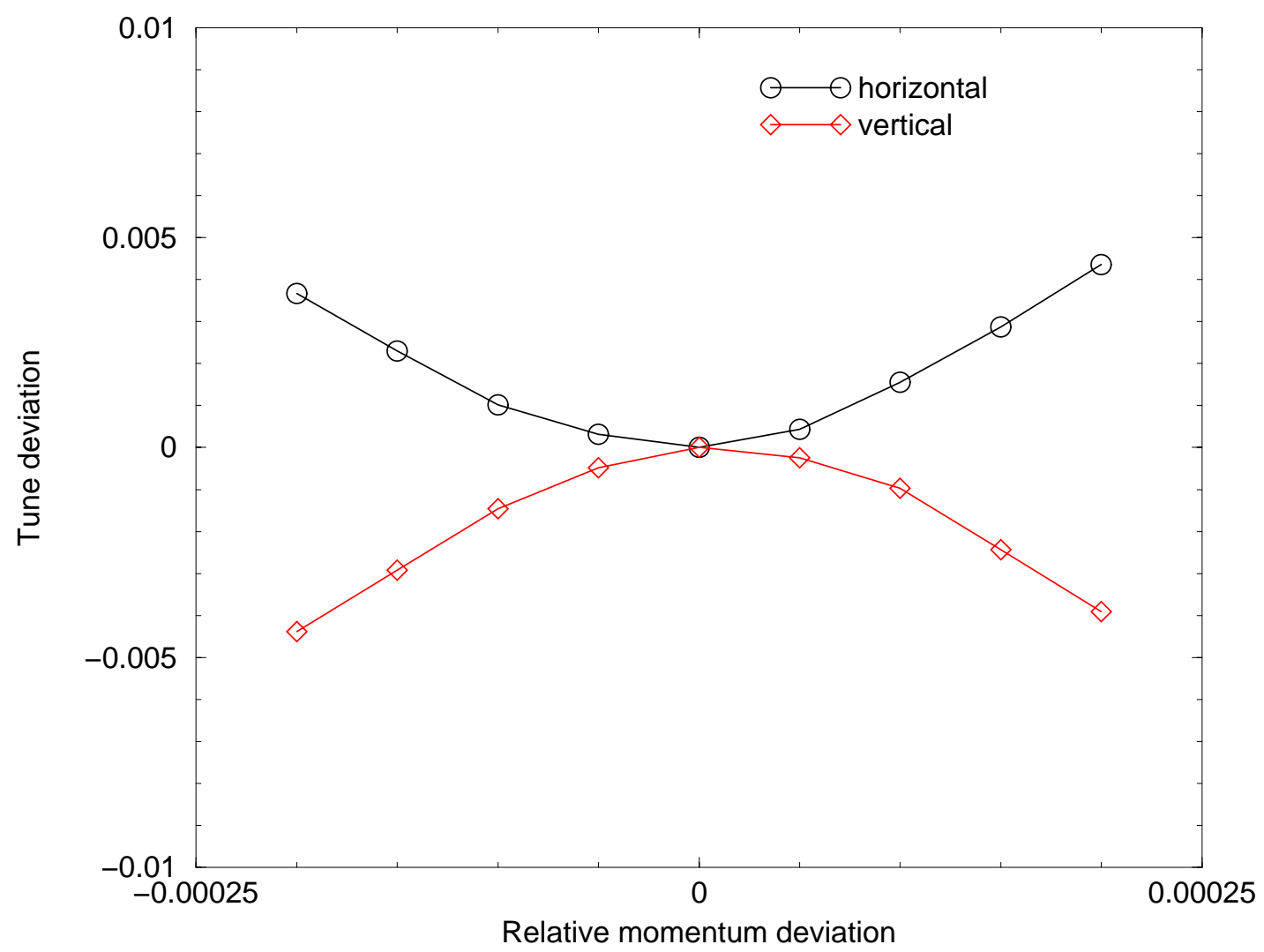

Figure 51: Horizontal and vertical tune as a function of the relative momentum deviation at a storage energy of $87.5 \mathrm{TeV}$. The horizontal scale corresponds to approximately $3 \sigma$ of the momentum distribution. 


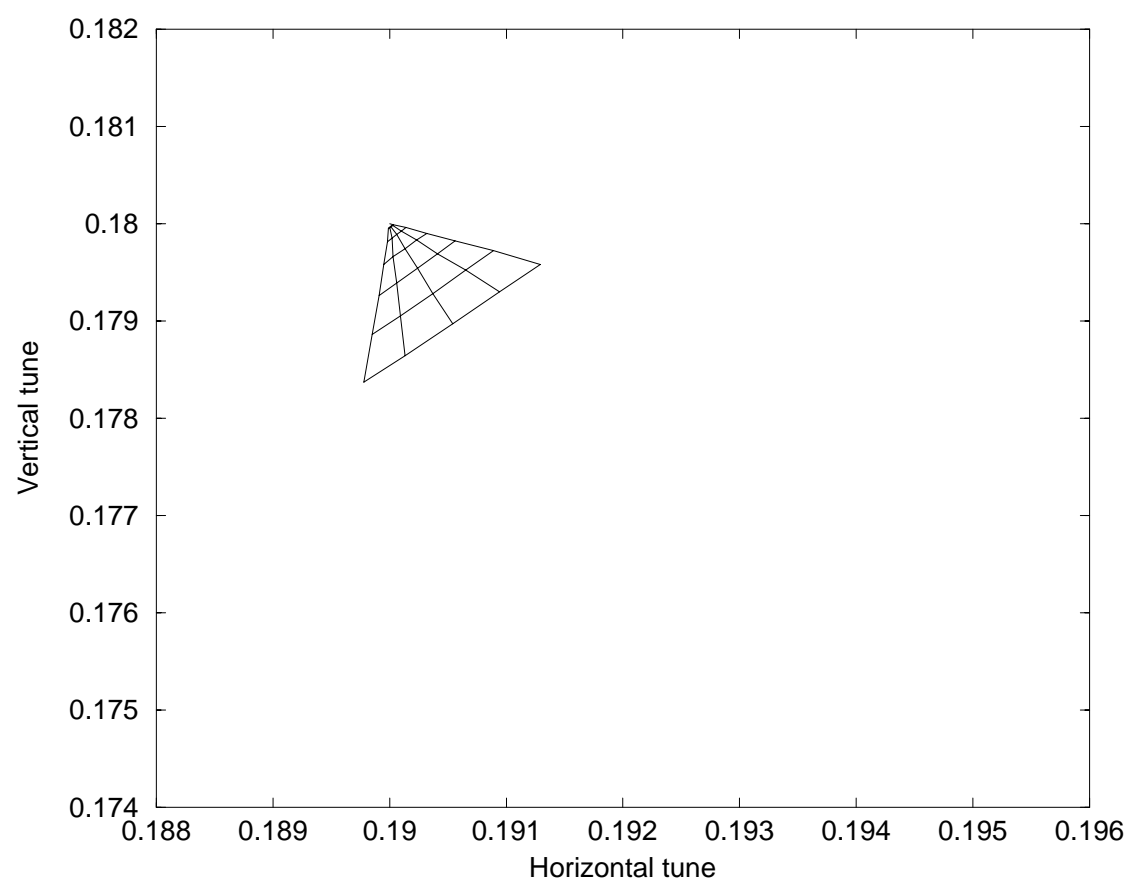

Figure 52: Tune space needed for on-momentum particles with betatron amplitudes of up to 6 sigma of the transverse rms beam size, without systematic octupole errors in dipoles. At a storage energy of $87.5 \mathrm{TeV}$.

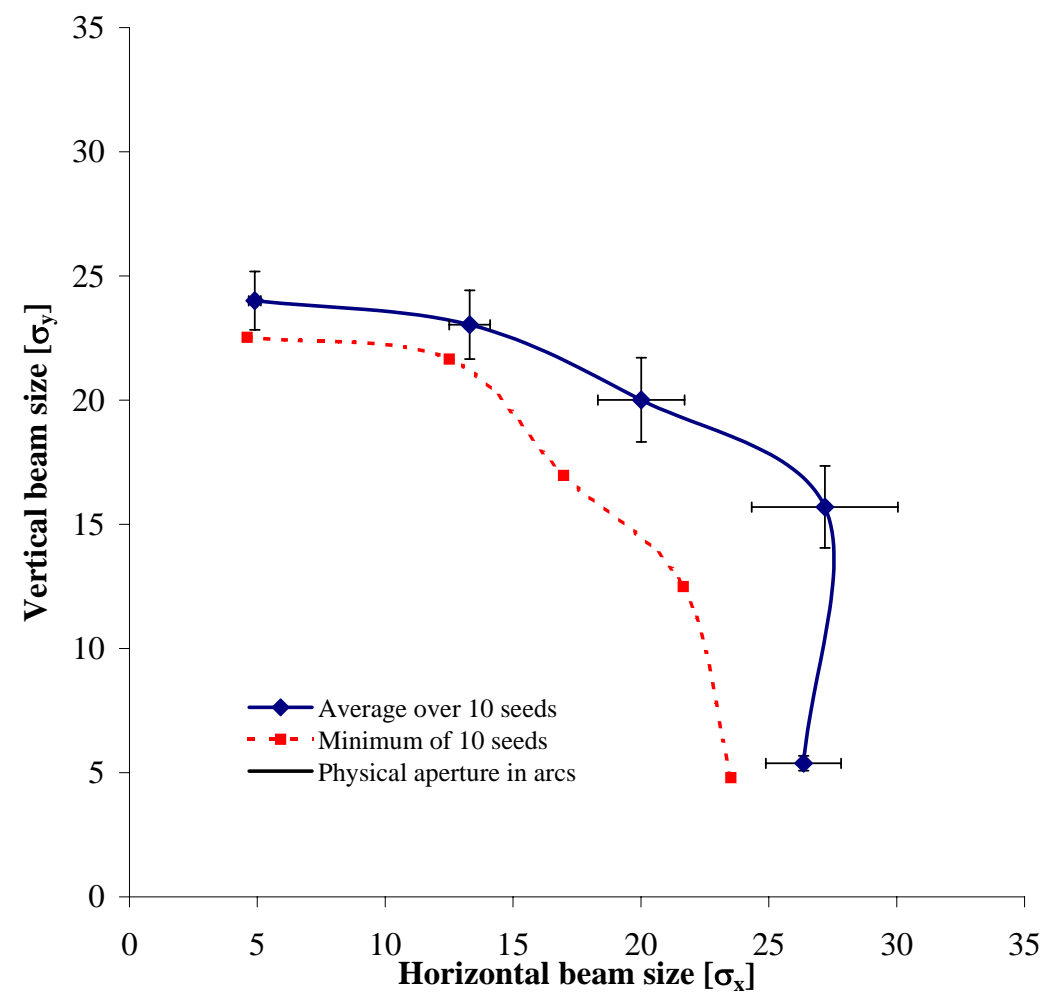

Figure 53: 1000-turn dynamic aperture at a storage energy of $87.5 \mathrm{TeV}$. Shown are the average over 10 seeds, the worst of 10 seeds, and the physical aperture, in units of the transverse rms beam size. The error bars of the average dynamic aperture are the rms value of 10 seeds. 


\subsection{Tolerances}

In this section we consider the effect of magnet misalignments as well as the effect of main dipole and quadrupole field errors on the beam orbit and betatron tunes. We discuss the tolerances for misalignment and field errors listed in Table 27. To this purpose we use the high-field storage optics with $\beta_{x}^{*}=3.7 \mathrm{~m}$ and $\beta_{y}^{*}=0.37 \mathrm{~m}$.

\begin{tabular}{|lrrl|}
\hline RMS ERROR & ARC & IR & \\
& & & \\
QUADRUPOLES & & & \\
Transverse offset & 0.25 & 0.2 & $\mathrm{~mm}$ \\
BPM to quad offset & 0.15 & 0.1 & $\mathrm{~mm}$ \\
Roll & 0.5 & 0.5 & $\mathrm{mrad}$ \\
Field Error $\Delta K / K$ & 0.5 & 0.1 & $10^{-3}$ \\
& & & \\
DIPOLES & & & \\
Roll & 0.5 & 0.5 & $\mathrm{mrad}$ \\
Field Error $\Delta B / B$ & 0.5 & 0.5 & $10^{-3}$ \\
& & & \\
\hline
\end{tabular}

Table 27: Tolerance summary for magnet alignment.

\subsubsection{Orbit Errors}

Possible sources of orbit error include transverse quadrupole misalignments (in both the horizontal and vertical directions), main dipole field integral errors (for the horizontal orbit), and roll of the main dipole magnets (for the vertical orbit). In order to estimate and compare the value of the orbit distortion coming from these different types of error sources, the rms closed orbit has been calculated. The calculations assumed the rms magnet misalignments listed in Table 27. Each of five different individual perturbation sources were considered in turn. In some cases the errors are introduced in all magnets in the accelerator arcs, while in others the errors are applied to the quadrupoles of one of the interaction region doublets.

The calculated results are summarized in Table 28. Horizontal (X) and vertical (Y) rms orbit values characterize the contribution coming from the different perturbation sources. The corrector strengths required to compensate for the perturbations are also listed. These strengths were calculated assuming that in the arc regions horizontal correctors are placed next to each focusing quad and vertical are next to defocusing quads. In the interaction region doublets there is one corrector per plane. The biggest effect comes from quadrupole offsets. The dipole integral field error and dipole roll errors quoted in Table 27 have little influence on the required corrector strength.

\begin{tabular}{|lcccc|}
\hline Error type & $\begin{array}{c}\text { X orbit } \\
\text { rms [mm] }\end{array}$ & $\begin{array}{c}\text { Required X strength } \\
\text { rms [Tm] }\end{array}$ & $\begin{array}{c}\text { Y orbit } \\
\text { rms [mm] }\end{array}$ & $\begin{array}{c}\text { Required Y strength } \\
\text { rms [Tm] }\end{array}$ \\
$\begin{array}{l}\text { Arc Quad offset } \\
\text { Arc dipole roll }\end{array}$ & 34 & 0.9 & 34 & 0.9 \\
Arc dipole field error & 5.7 & 0.3 & 5 & 0.25 \\
IR Quad offset & 9 & 3.4 & 12 & 3.4 \\
IR dipole field error & 0.7 & 0.04 & & \\
\hline
\end{tabular}

Table 28: RMS horizontal and vertical dipole correctors strengths required to correct the closed orbit under the influence of different error sources. (BPM-to-Quad misalignment effect is included in quad offset.) 


\subsubsection{Tune Error, Coupling and IR Chromatic Effects}

The betatron tune shift caused by an rms quadrupole field error of $10^{-4}$ and the betatron coupling effect produced by rms quadrupole rolls of $1 \mathrm{mrad}$ have been calculated. Table 29 shows the effect produced by quadrupole gradient errors, while Table 30 shows the coupling effect produced by quadrupole rolls.

The tolerance for arc quadrupole error can be put at $5 \times 10^{-4}$ providing a $2.5 \mathrm{rms}$ beta-beating value of about $9 \%$ is acceptable. A small number of quadrupole correctors placed in the arcs will easily compensate for this beta-beating. Field errors in interaction region quadrupoles can be corrected by adjusting the currents of power supply for these quads. After the current adjustment is done the current fluctuations should be kept well below the $10^{-4}$ level. Both global correction in the arcs and local skew quadrupole correction in the interaction regions are required for the compensation of betatron coupling effects. With these correction systems in place it is possible to relax the quad roll tolerances to about $0.5 \mathrm{mrad}$.

\begin{tabular}{|lcc|}
\hline & $\mathrm{rms} \Delta Q\left[10^{-3}\right]$ & $\mathrm{rms} \Delta \beta / \beta[\%]$ \\
All arc quads & 1.15 & 0.75 \\
IR doublet quads & 3.0 & 3.26 \\
\hline
\end{tabular}

Table 29: The effects of quadrupole gradient errors with an rms value of $\Delta K / K=10^{-4}$.

\begin{tabular}{|lc|}
\hline & $\mathrm{rms} \Delta Q_{\min }\left[10^{-3}\right]$ \\
All arc quads & 11.4 \\
IR doublet quads & 30.0 \\
\hline
\end{tabular}

Table 30: The betatron coupling effect due to an rms quadrupole roll angle of $1.0 \mathrm{mrad}$.

Because of the very large maximum $\beta$ values in the interaction region quadrupoles, the chromatic effects produced by these quadrupoles have been evaluated. For the design value of rms momentum spread of $5 \times 10^{-5}$ the amplitude of the chromatic vertical beta-wave was found to be just a few percent. Thus the effect is not large, mainly due to the small rms momentum spread. The vertical chromaticity produced by the quadrupoles from one interaction region has been found to be about -42 . The horizontal chromatic effects are smaller than the vertical.

\subsubsection{Corrector Strengths}

The maximum dipole corrector strengths displayed in Table 31 are 2.5 times the rms value when all error sources are combined in quadrature. Assuming a maximum achievable field of $3.5 \mathrm{~T}$ for the IR dipole correctors, and $2.2 \mathrm{~T}$ for the arc dipole correctors, magnetic lengths of $1.05 \mathrm{~m}$ in the arcs and $2.5 \mathrm{~m}$ in the IRs are required. If the maximum corrector strengths are increased to higher values than those cited in Table 31, then the corresponding tolerances in Table 27 can be relaxed accordingly.

Even the maximum dipole corrector strengths listed in Table 31 can not make up for the magnet offset diffusion caused by the ground motion that is described elsewhere in this report. Ground motion might put magnet offsets well beyond the defined tolerances after few years of movement. Thus, periodic magnet realignment will be necessary. Some means to simplify the realignment procedure, such as using stepping motors, should be evaluated.

\subsection{Corrections}

The high field VLHC collider is a machine that relies on powerful diagnostics and correction systems to reach its ultimate performance, similarly to existing and planned hadron colliders like RHIC and the LHC. Static corrections of trajectory, orbit, optical errors and distortions are required as well as dynamic corrections of machine characteristic during ramping and beta squeeze. The effectiveness of feedback systems has to be evaluated with respect of the tightening of tolerances. In the present chapter we will specify a correction system for VLHC on the basis of the error tolerances worked out for the VLHC itself and 


\begin{tabular}{|lrl|}
\hline ARC CORRECTORS & & \\
Dipole magnetic length & 1.05 & $\mathrm{~m}$ \\
Dipole max strength & 2.3 & $\mathrm{Tm}$ \\
Skew quad length & 0.95 & $\mathrm{~m}$ \\
Skew quad max strength & 95 & $\mathrm{~T}$ \\
Sextupole magnetic length & 0.95 & $\mathrm{~m}$ \\
Sextupole max strength, $L\left(d^{2} B / d x^{2}\right)$ & 7400 & $\mathrm{~T} / \mathrm{m}$ \\
& & \\
IR CORRECTORS & & \\
Magnetic length & 2.5 & $\mathrm{~m}$ \\
Dipole max strength & 8.6 & $\mathrm{Tm}$ \\
Skew quad max strength & 95 & $\mathrm{~T}$ \\
& & \\
\hline
\end{tabular}

Table 31: Corrector magnetic lengths and strengths. The maximum strengths are 2.5 times the rms value required when all contributions are added in quadrature.

experience from recently built or designed hadron colliders. The following two sections describe the arc correction system, and the special correction requirements of the interaction regions.

\subsubsection{Arc Correction System}

The arc correction system consists of an orbit correction system, a coupling correction system and a chromaticity correction system. The correction plan is to use the arc systems to correct the injection configuration, with enough strength to propagate the corrections to top energy. Additional errors and effect arising from the collision optics and configuration are addressed by the Interaction region (IR) system. This strategy has been successfully used in RHIC. Preliminary simulation results with the VLHC injection optics and predicted field imperfections lead to the conclusion that there is no need for nonlinear correctors in the arc cells other than the chromaticity sextupoles. The orbit correction system consists of dipole correctors placed next to each arc quadrupole. The configuration of the arc corrector packages (dipole, sextupole) and the tolerances were already discussed in the previous chapters. The specification for the corrector strengths are listed in Table 31 .

Automatic orbit correction, based on a menu of algorithms, will be provided, based on fast orbit acquisition from the beam position monitor system, including turn-by-turn acquisition. Sextupole correctors are also part of the arc corrector package, to statically compensate the chromatic effect and dynamically compensate for time dependent effect in the magnets.

For the correction of coupling the proposed solution has the skew quadrupoles located at QF and QD positions in the dispersion suppressor cells, in the 2 cluster regions in the interaction region areas. The skew quadrupole replace the sextupole corrector in the arc correction package configuration, for a total of 80 skew quadrupole correctors per ring. The skew quadrupoles can be connected in up to 4 families, which allows flexibility in choosing the method for coupling compensation. The system specifications have been determined by evaluating the leading source of coupling in the arcs, the roll misalignment error in the arc quadrupoles, and allowing some margin for the compensation of skew quadrupole error fields in the dipoles.

The specification for the skew quadrupole integrated strength in the corrector package, $95 \mathrm{~T}$, has been used to compare the performance of the VLHC skew quadrupole correction system to the similar RHIC system. As a figure of merit, we take R= (total skew corrector strength) $/[\sqrt{N} *$ (arc quad strength) $]$ with the numerator being the total skew corrector strength available in the machine and the denominator proportional to the minimum tune separation generated by a distribution of random roll errors in the arc quadrupoles.

The ratio for RHIC (with $1.5 \mathrm{~T}$ integrated strength in the skew quadrupoles, 48 skew quadrupoles per ring, and 276 arc quadrupoles at $7.81 \mathrm{~T}$ each) is 0.56 . For the VLHC ( 80 skew quadrupoles at $95 \mathrm{~T}$ and 784 arc quadrupoles, $8 \mathrm{~m}$ long and a gradient of $400 \mathrm{~T} / \mathrm{m}$ ) the ratio is 0.18 , about a factor 3 from RHIC. Given that the roll misalignment specification for RHIC is 1 $\mathrm{mrad}$ in the arc quadrupoles, the proposed skew correction system of the VLHC can adequately compensate a random alignment error in the arc quadrupoles of $250 \mu \mathrm{rad}$, with some margin for other sources of arc coupling. Coupling effects arising from the interaction regions are corrected with the IR decoupling system and will be discussed in the next section. 


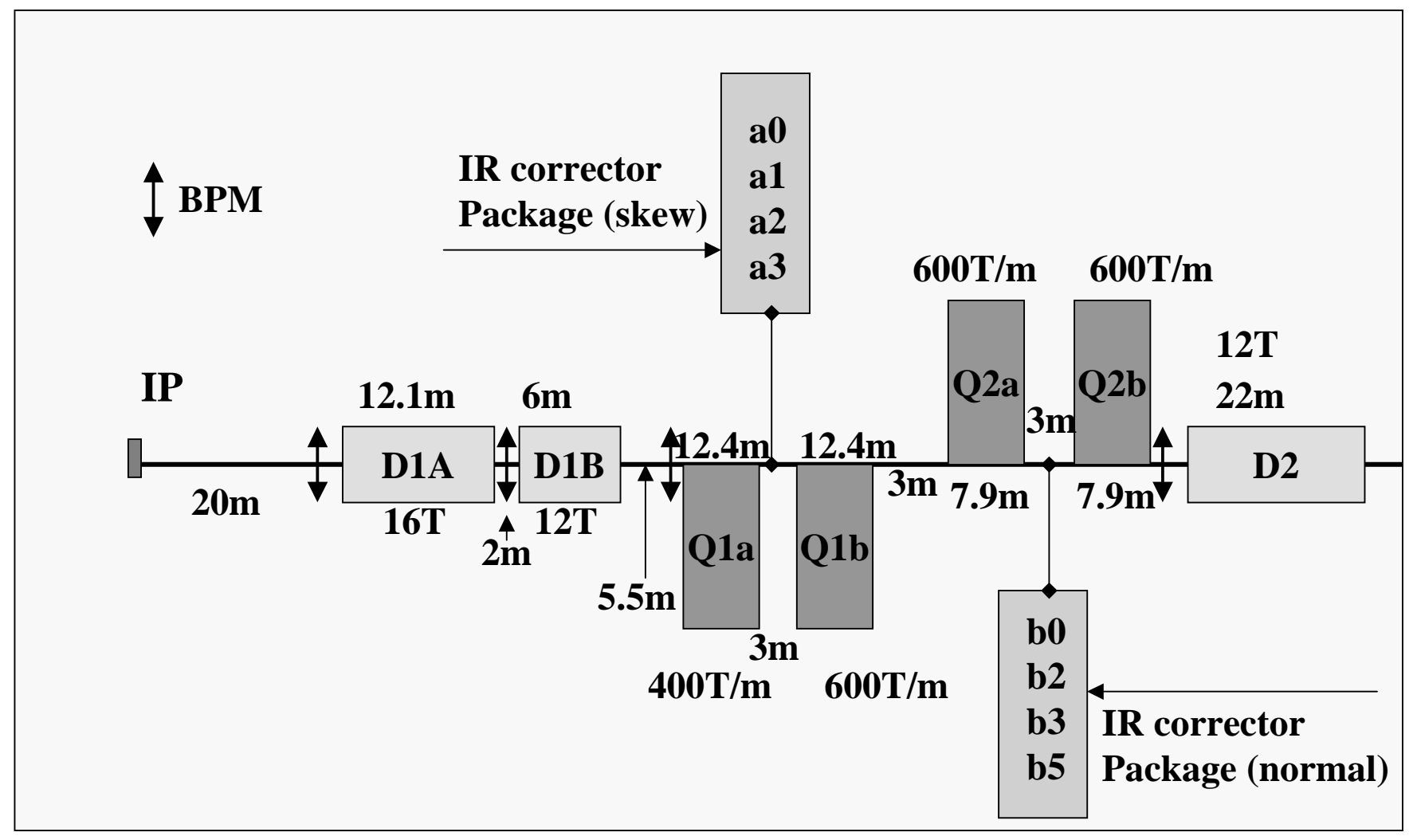

Figure 54: Schematics of the interaction region correction system.

\subsubsection{Interaction Region Correction System}

The goal of the IR system is to locally correct for effects and errors arising from the interaction regions. The fact that the effect of the IRs is most relevant in collision optics, when the focusing at the interaction point (IP) and the beat functions in the IR final focus quadrupoles are maximized, is what makes this correction modularity possible. The independent and local correction of IR effects has been a guiding principle in the design of RHIC and the LHC.

The IR correction systems can be functionally divided in linear and nonlinear, but the implementation often favors integrated solutions, like multi-layer correction packages, given the tight constraints on space near the IP. The schematics for the proposed IR correction system is displayed in Figure 54.

Two correction packages are placed in the $3 \mathrm{~m}$ drifts between the two doublet quadrupoles, a skew package between Q1A and Q1B, and a normal package between Q2A and Q2B to take advantage of the beta functions. A detailed magnetic design of the IR region correction system is in progress, so the specifications are driven from the requirements and extrapolation from the correction package designed for the arcs. The linear correction system consists of 2 dipole correctors and 1 skew quadrupole layer. The dipole correctors provide orbit control, IP and crossing angle control. An integrated strength of $8.6 \mathrm{Tm}$ will correct for the tolerances listed in Table 27. Feedback on beam position at the IP and on the crossing angle may be required and will be addressed in the future.

The skew quadrupole corrector is used to compensate locally the residual coupling from the final focus doublet quadrupoles. Without local correction a tolerance of $0.1 \mathrm{mrad}$ on the doublet alignment would be required to keep the minimum tune separation $\Delta Q_{\min }<0.003$. An uncorrected tolerance of $0.1 \mathrm{mrad}$ is consistent also with extrapolation from LHC and SSC. With an integrated strength of $95 \mathrm{~T}$ in the local skew quadrupole at the doublet it is possible to compensate for a residual misalignment of $0.5 \mathrm{mrad}$, thus relaxing the alignment tolerance. Further optimization between roll alignment tolerance and local skew quadrupole strength will be necessary.

The nonlinear correction system is modeled after the design of the RHIC and LHC systems, adapted to the doublet optics configuration, and with consideration to the design of the VLHC doublet quadrupoles. A local sextupole, octupole and a dodecapole corrector are part of the normal corrector package, and a skew sextupole and octupole are in the skew package. The 
choice of layers is dictated by previous experience with RHIC and LHC and from preliminary values of the doublet harmonics. A more detailed study will be necessary to validate these choices and to determine the strengths of the nonlinear correction layers. With the present technology a 4-layer corrector is at the limit of feasibility. Should additional or stronger correctors be necessary, they can be installed in the drift between Q1B and Q2A or between Q2B and D2.

The VLHC IRs with the nominal optics contribute -42 units of vertical chromaticity to a machine that has a natural chromaticity of less than -200 units. The need for a local chromaticity compensation system to correct for the second order chromaticity from the IRs will have to be addressed. To this end the sextupole corrector at the doublet is useless since it is in a zero dispersion location. Should IR second order chromaticity compensation be necessary that can be achieved with 2 families of sextupoles placed in the arc region adjacent to the IRs. The first family SX1 compensates the second order IR chromaticity, the second family SX2 is necessary to suppress the first order chromaticity contributed by SX1.

Double plane beam position monitors (BPMs) are essential in the interaction region and they have to be included in the planning from the start. The BPM in front of D1A is the main tool to monitor the IP and must have capability of observing both beams. The other BPMs are used for orbit and crossing angle monitoring.

\subsection{Beam Stability}

Table 32 lists fairly well known parameters relevant to beam stability. Unknown parameters include the broad band impedance as well as narrow band parasitic impedances. For the latter quantities the stability calculations will be used to obtain safe limits.

\begin{tabular}{|lll|}
\hline & INJECTION & INITIAL STORE \\
PARAMETER & & \\
circumference & $2 \pi R=233 \mathrm{~km}$ & \\
kinetic energy & $10 \mathrm{TeV}$ & $87.5 \mathrm{TeV}$ \\
RF Voltage & $50 \mathrm{MV} / \mathrm{turn}$ & $200 \mathrm{MV} / \mathrm{turn}$ \\
RF frequency & $488 \mathrm{MHz}$ & \\
synchrotron frequency & $f_{s}=3.48 \mathrm{~Hz}$ & $f_{s}=2.35 \mathrm{~Hz}$ \\
rms bunch length & $\sigma_{t}=0.273 \mathrm{~ns}$ & $\sigma_{t}=0.112 \mathrm{~ns}$ \\
nominal betatron tunes & $Q_{x}=218.19, Q_{y}=212.18$ & \\
beam pipe radius & $b=1 \mathrm{~cm}$ & $1.5 \mu \mathrm{m}$ \\
rms normalized transverse emittance & $1.5 \mu \mathrm{m}$ & $Q_{x}^{\prime}= \pm 2, Q_{y}^{\prime}= \pm 2$ \\
nominal chromaticity & $Q_{x}^{\prime}= \pm 2, Q_{y}^{\prime}= \pm 2$ & \\
revolution frequency & $f_{0}=1.286 \mathrm{kHz}$ & \\
$\gamma_{t}$ & 194.13 & \\
rms longitudinal emittance & $\pi \sigma(E) \sigma(t)=2.0 \mathrm{eV}-\mathrm{s}$ & \\
bunches for symmetric fill & $M=41280$ & \\
protons/bunch & $9.0 \times 10^{9}$ & \\
& & \\
\hline
\end{tabular}

Table 32: Parameters relevant to beam stability.

Start by considering the situation at injection energy. Transverse coupled bunch stability is largely determined by the resistive wall impedance. With its large circumference the relevant frequency range for VLHC starts at $\sim 1 \mathrm{kHz}$. The skin depth is given by

$$
\delta=\sqrt{\frac{2 \rho_{e}}{\mu|\omega|}}
$$

where $\rho_{e}$ is the electrical conductivity, $\mu$ is the magnetic permeability, and $\omega=2 \pi f$ is the angular frequency, with all quantities in MKS. For pure copper at $80 \mathrm{~K}$ the electrical resistivity due to phonon scattering is $\rho_{e}=1 / \sigma_{c}=1.6 \times 10^{-9} \Omega \mathrm{m}$ [48]. There is an additional component to the resistivity due to impurity scattering which remains to be determined. For cold stainless steel (SS) the conductivity is $\rho_{s}=1 / \sigma_{s}=7.3 \times 10^{-7} \Omega \mathrm{m}[49]$. For a frequency of $f=1 \mathrm{kHz}$ the skin depth in cold copper is $\geq 0.64 \mathrm{~mm}$, while for cold SS it is $1.4 \mathrm{~cm}$. The $\geq$ for the copper is due to neglecting impurity scattering. To reduce uncertainties 
the copper lining will be neglected and the resistive wall transverse impedance it taken to be

$$
Z_{\perp, r w}=\frac{R c(1-i \operatorname{sgn}(\omega))}{2 \omega b^{3} \sigma_{s} \delta_{s}}
$$

where we have assumed a time dependence $\propto \exp (-i \omega t)$. Note that the resistive wall impedance will probably dominate the transverse impedance so calculating the effect of the copper liner should take precedence over estimating the impact of the myriad small additions to the transverse impedance.

The coherent betatron frequency in the weak coupling limit of the Wang formalism[50] is

$$
\Delta Q_{m}=m \omega_{s}-i \frac{\bar{I} c}{4 \pi 2^{|m|}|m| !\left(E_{T} / q\right) Q_{\beta}} \sum_{n=-\infty}^{\infty} Z_{\perp}\left[(n M+s) \omega_{0}+\omega_{\beta}\right] e^{-\tilde{n}^{2} \sigma^{2}}(\tilde{n} \sigma)^{2|m|},
$$

where $m=\ldots,-1,0,1, \ldots$ is the synchrotron mode number, $\omega_{s}$ is the angular synchrotron frequency, $\bar{I}$ is the average current, $M$ is the number of bunches, $s$ is the coupled bunch mode number, $E_{T} / q$ is the total proton energy divided by its charge, $\sigma$ is the rms bunch length in units of machine azimuth, $\tilde{n}=n M+s+Q_{\beta}-Q_{\beta}^{\prime} / \eta$, and $\eta=1 / \gamma_{T}^{2}-1 / \gamma^{2}$ is the slip factor.

Equation (107) assumes $M$ identical, equally spaced bunches so it neglects the effect of the abort gap. This is a standard formula that can be found in ZAP, Chao's book, or any number of papers. Figure 55 shows the real (dQr) and imaginary (dQi) parts of the tune shift for $m=Q_{\beta}^{\prime}=0$ at the injection energy.

The maximum growth rate with $\operatorname{Im}\left(\Delta Q_{0}\right)=0.05$ occurs for the lowest negative sideband which would have an observed frequency of $0.8 f_{0}=1.03 \mathrm{kHz}$. The growth rate of $0.4 / \mathrm{ms}$ corresponds to an e-folding time of 3.2 turns. This mode and several other low frequency modes need to be damped. Following Marriner's suggestion [51] assume a transverse pickup which is low pass filtered, amplified, and applied to a kicker $1 / 4$ of a betatron wavelength downstream. For low frequencies, beam position monitors differentiate the input signal, which is the product of the instantaneous current with the offset. A simple model produces the effective transverse impedance

$$
Z_{\perp, d}(\omega)=-Z_{c} \frac{i f}{f_{0}}\left(\frac{1}{1-i f / f_{d}}\right)^{2} .
$$

Setting $f_{d}=5 \mathrm{kHz}$, and choosing $Z_{c}$ so that the fastest damped mode has $d Q i=-0.4$, in the absence of the resistive wall impedance, yields the growth shown in Figure 56. With the damper, the growth rate of the most unstable mode is a factor of 20 smaller than with no damper. It is assumed that a bunch by bunch damper with one turn delay, or similar device, can take care of any residual, rigid mode, coupled bunch instability.

Next consider the fast head tail instability. This is done by choosing a tune $Q_{y}=212.5$ with $M=41281$ and $s=20428$. These numbers satisfy $s+Q_{y}-M=-\left(s+Q_{y}\right)$ which gives growth rates identically equal to zero for the weak coupling limit. In this case instability occurs when the impedance causes the coherent frequencies of adjacent synchrobetatron sidebands to collide. The formulas may be found in [52]. They appear to be identical to the MOSES code [53] but no direct check has been performed. Figure 57 shows the fast head-tail threshold at injection energy. The design current is safe by about a factor of 2 even with the assumption of no copper liner. With the copper liner the tune shifts should be substantially smaller so an rf voltage of $50 \mathrm{MV} /$ turn is probably fine.

The last transverse instability to consider is the normal head-tail instability. We limit our attention to modes that are damped by the low frequency damper, using $s=-220$ as a test case. Using the weak coupling formulas there is no instability predicted for $0 \leq Q_{y}^{\prime} \leq 2$ at injection energy, which agrees well with experience. With mode coupling between the 3 lowest synchrotron modes, as per Figure57, the same range of chromaticities are, again, stable. With coupling between the 6 modes with $|m| \leq 2$ a weak instability at injection energy with $d Q i \lesssim 10^{-5}$ is found. The growth rate depends slightly on chromaticity but some growth is always predicted. Predictions for other accelerators are similar, with no instability showing up in the real machine. More work is needed to understand this but it is likely that synchrotron or betatron tune spread will Landau damp the higher order unstable modes.

Longitudinal instabilities at injection and during the ramp will not be a problem if the impedance is kept small enough. Since transition is not crossed the broad band impedance will be an issue only if the coherent tune shift is large enough to cause undamped coherent oscillations. These oscillations are not in themselves a problem, but will allow small parasitic resonances to drive unstable modes. Therefore, the broad band impedance is constrained by demanding that coherent dipole oscillations are damped by the synchrotron tune spread. This remains to be done.

Transverse instabilities at store are more interesting. Figure 58 shows the growth rates of the $m=0$ modes at the beginning of store. The most unstable mode has $\operatorname{Im}(\Delta Q)=0.0025$, which is significantly larger than the synchrotron tune. If this mode is present it must be actively damped. 
Figure 1

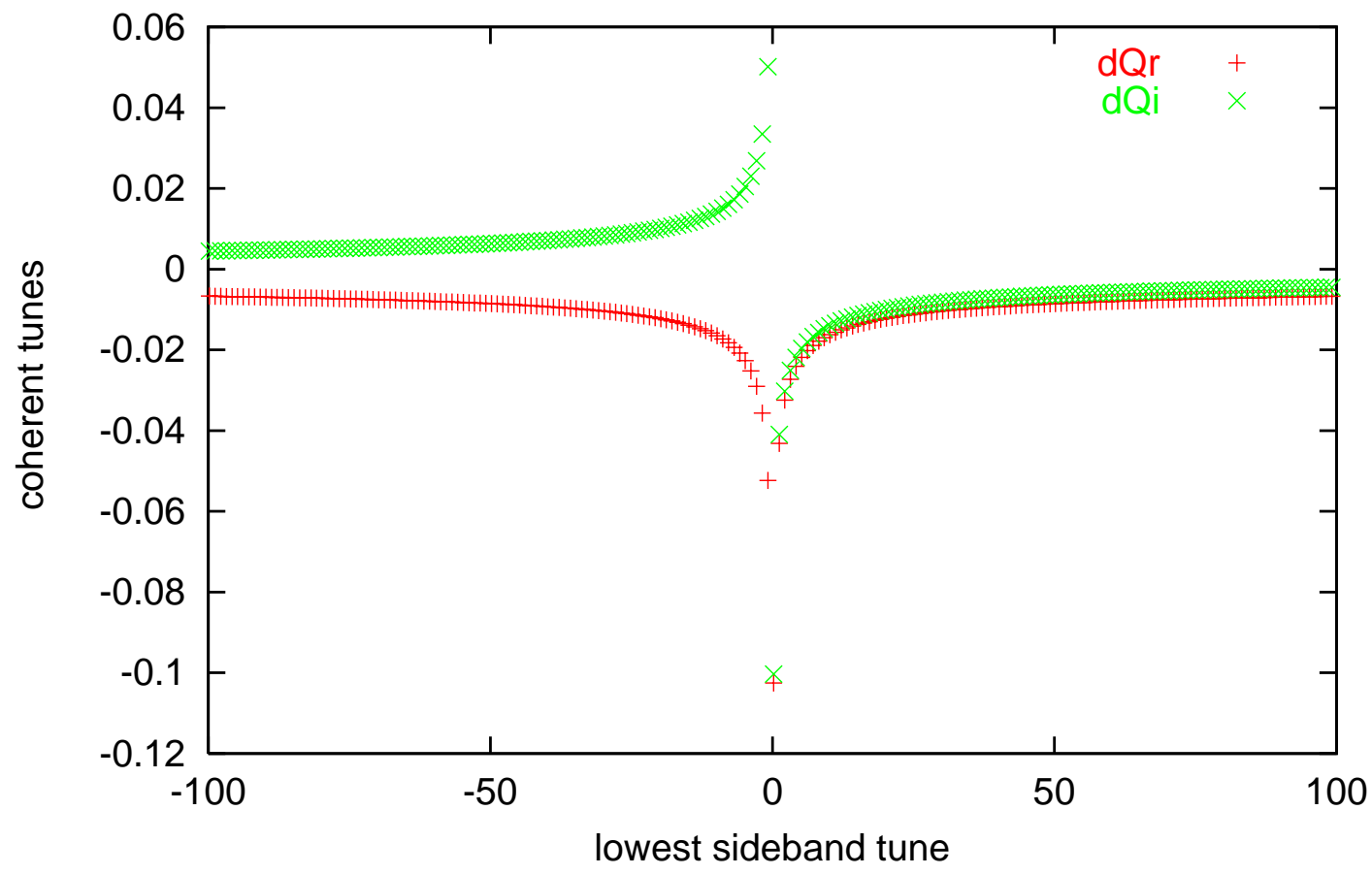

Figure 55: Coherent tunes for $\mathrm{m}=0$ at $10 \mathrm{TeV}$ versus observed frequency of lowest betatron sideband in units of the revolution frequency $(1.29 \mathrm{kHz})$. There is no damper.

Figure 2

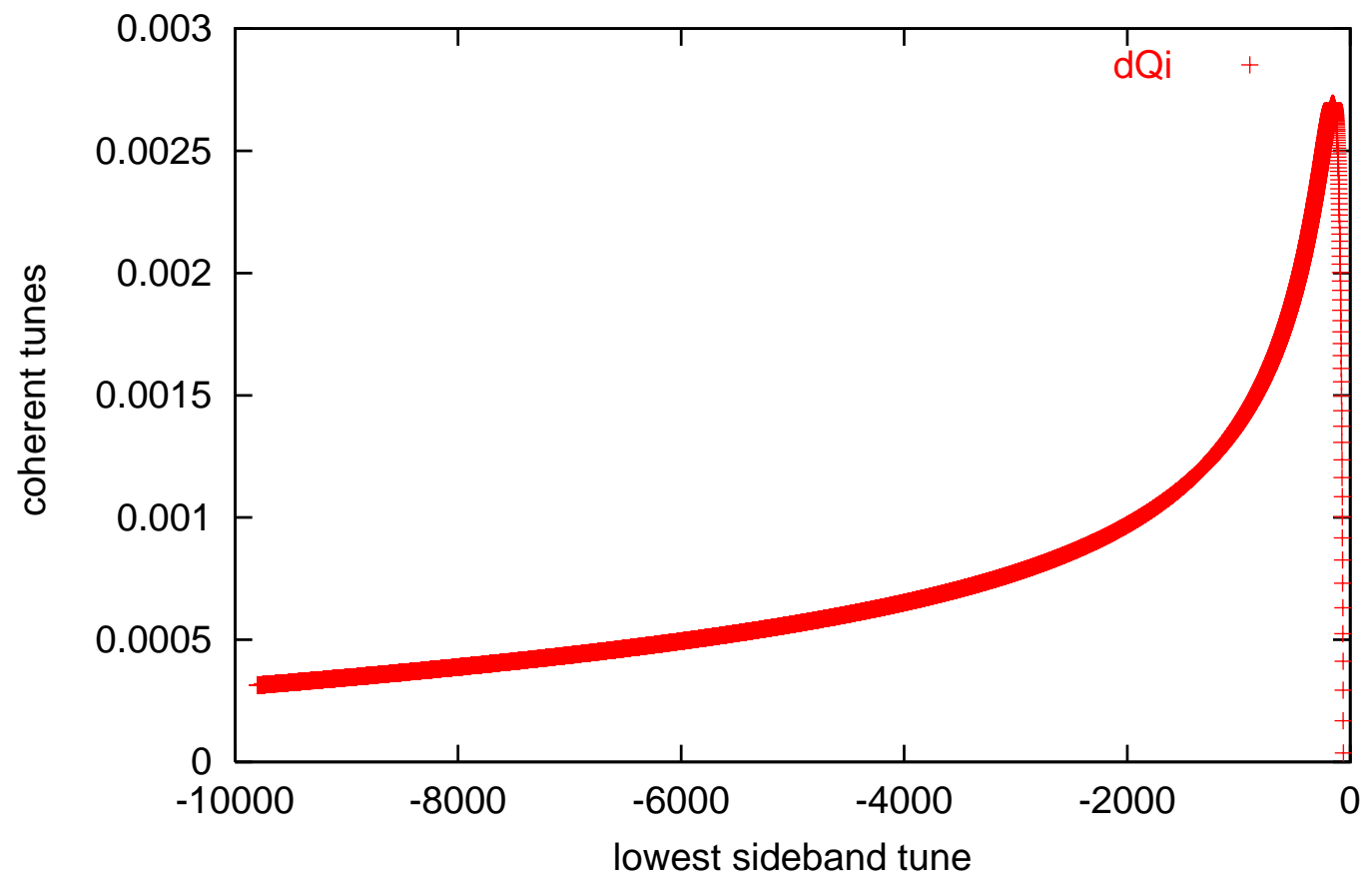

Figure 56: Imaginary part of unstable coherent tunes for $\mathrm{m}=0$ at $10 \mathrm{TeV}$ versus observed frequency of lowest betatron sideband in units of the revolution frequency $(1.29 \mathrm{kHz})$. The characteristic damper frequency is $5 \mathrm{kHz}$ and gain corresponding to $d Q r=$ -0.4 at the strongest damped sideband. 


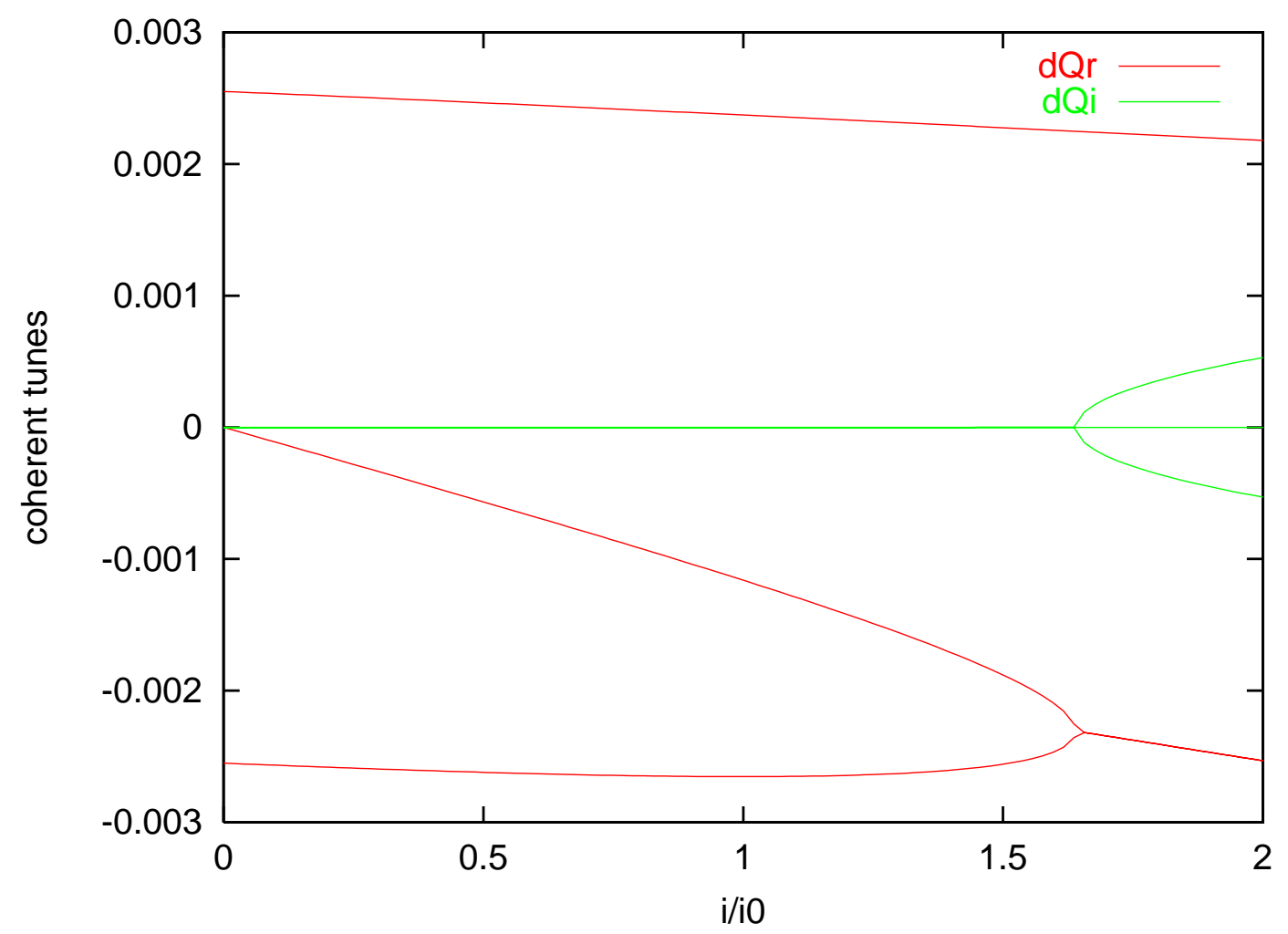

Figure 57: Growth rates for fast head-tail at $10 \mathrm{TeV}$ versus bunch current in $10^{10}$ protons.

Figure 4

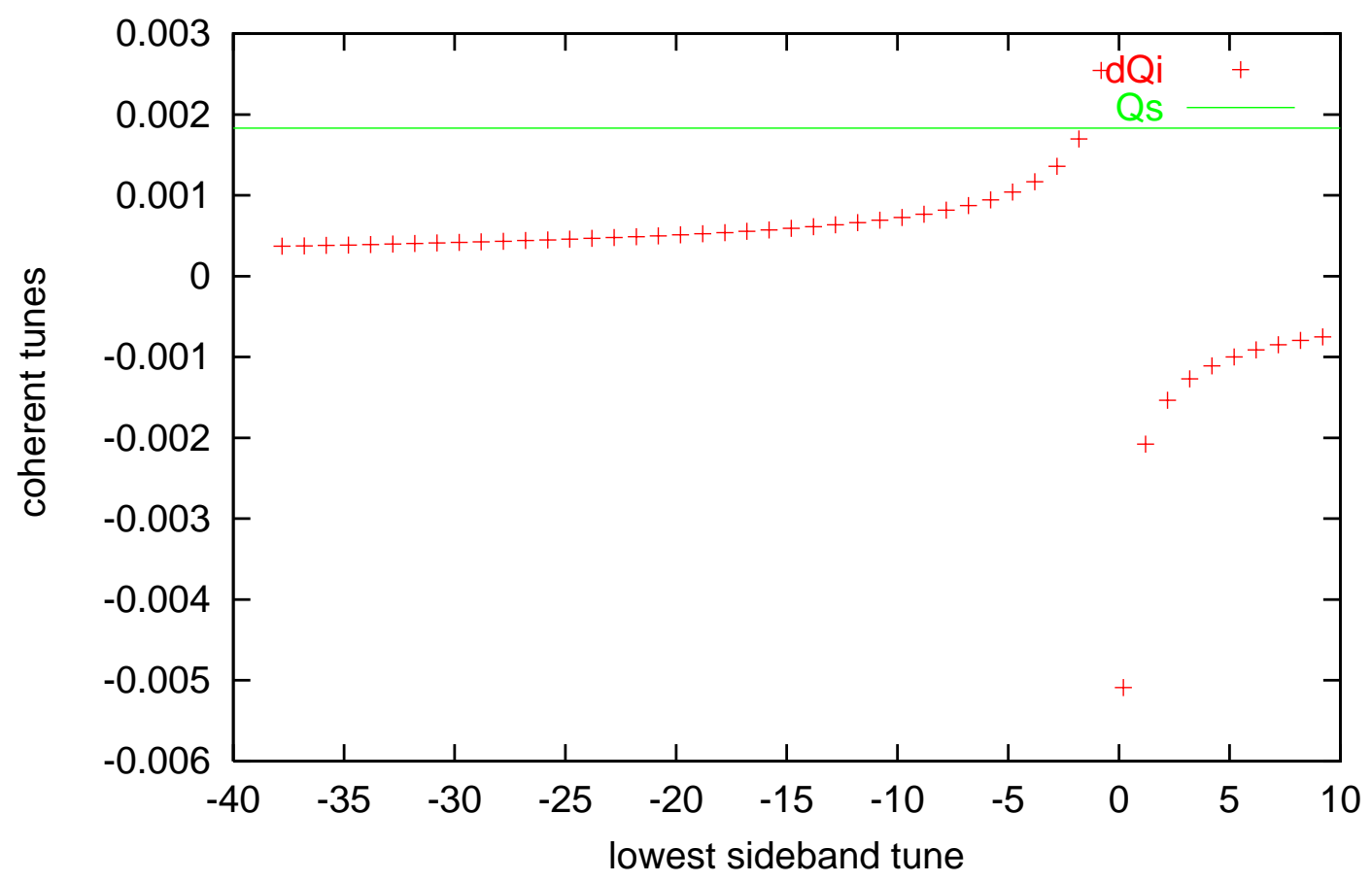

Figure 58: Imaginary part of unstable coherent tunes for $\mathrm{m}=0$ and $87.5 \mathrm{TeV}$ versus observed frequency of lowest betatron sideband in units of the revolution frequency $(1.29 \mathrm{kHz})$. The horizontal line is the synchrotron tune for $200 \mathrm{MV} /$ turn. 


\subsection{Synchrotron Radiation}

Proton beams in the VLHC emit synchrotron radiation just as electrons do in electron storage rings. An important advantage of synchrotron radiation in the high energy ring is that the significant damping stabilizes the beam dynamics, shrinks the emittances, enables flat beam collisions, and tends to make store performance independent of injection errors. On the other hand, disadvantages include a large power load to the cryogenic system, photon induced gas desorption, and the possibility of electron cloud instability [54].

The critical wavelength characterizing the synchrotron radiation spectrum in a dipole of bend radius $\rho=29.9 \mathrm{~km}$ is

$$
\lambda_{c}=\frac{4 \pi \rho}{3 \gamma^{3}}=1.543[\mathrm{~A}]
$$

where $\gamma=9.33 \times 10^{4}$ is the Lorentz factor corresponding to the storage energy of $E=87.5 \mathrm{TeV}$. Photons are emitted tangential to the proton path within a small angle $\theta \sim 1 / \gamma \approx 10 \mu \mathrm{rad}$. The vast majority of photons (91\%) have energies smaller than the critical energy, given by

$$
E_{c}[\mathrm{keV}]=\frac{1.239}{\lambda_{c}[\mathrm{~A}]}=8.03[\mathrm{keV}]
$$

Nonetheless, the critical energy divides the spectrum in half with respect to the dissipated power.

The energy loss per particle per turn is

$$
U_{0}=\frac{C_{g} E^{4}}{2 \pi} C<G^{2}>=15.3[\mathrm{MeV}]
$$

where $C$ is the total circumference, the constant $C_{g}=7.783 \times 10^{-18} \mathrm{~m} \mathrm{GeV}^{-3}$ for protons, and $G=1 / \rho$ is the dipole bending strength. Angle brackets $\langle>$ denote an average over the entire design trajectory circumference in the general case when there is more than one bend radius. However, the VLHC is isomagnetic - has only has one bend radius - and so

$$
<G^{n}>=\frac{1}{\rho^{n}}
$$

The number of photons emitted per proton per turn is

$$
N_{\gamma}=\frac{15 \sqrt{3}}{8} \frac{U_{0}}{E_{c}}=6188
$$

and so the total photon rate per unit length in a dipole is

$$
\frac{d N}{d s}=2.56 \times 10^{17} I=1.76 \times 10^{16}[\text { photons } /(\mathrm{m} \mathrm{s})]
$$

where the nominal beam current is $I=68.9 \mathrm{~mA}$. The total synchrotron power radiated in a single ring is

$$
P=U_{0} I=1.05[\mathrm{MW}]
$$

for a two ring total of $2.1 \mathrm{MW}$.

The exponential damping times for the amplitudes of horizontal, vertical, and longitudinal oscillations are given by

$$
\tau_{x, y, s}=T_{0} / J_{x, y, s}
$$

where the characteristic time $T_{0}=2 T\left(E / U_{0}\right)$ is simply related to the revolution period, $T$. Natural partition number values $\left(J_{x}, J_{y}, J_{s}\right)=(1,1,2)$ are assumed. The equilibrium rms momentum width is

$$
\left(\frac{\sigma_{p}}{p}\right)^{2}=\frac{C_{q} \gamma^{2}}{J_{s}} \frac{<G^{3}>}{<G^{2}>}
$$

while the natural normalized rms horizontal emittance is

$$
\epsilon_{x} \equiv \gamma \frac{\sigma^{2}}{\beta}=\frac{C_{q} \gamma^{3}}{J_{x}} \frac{<G^{3} H>}{<G^{2}>}
$$


where $C_{q}=2.087 \times 10^{-16} \mathrm{~m}$ and $H$ is a property of the FODO cell optics

$$
H=\gamma \eta^{2}+2 \alpha \eta \eta^{\prime}+\beta \eta^{2}
$$

where $\alpha, \beta, \gamma$, and $\eta$ are Twiss functions.

Note that $U_{0} \sim B \gamma^{3}$ and $T_{0} \sim 1 /\left(B^{2} \gamma\right)$, where $B$ is the dipole field, independent of the lattice optics structure. The momentum width is also independent of the lattice structure, and scales like $\sigma_{p} / p \sim \sqrt{B \gamma}$. By contrast, the natural horizontal emittance depends strongly on the lattice, scaling like $\epsilon_{x} \sim B^{3} L^{3}$, where $L$ is the half length of a FODO cell. The normalized emittance is independent of energy!

Electron storage rings all operate with a room temperature beam environment. By contrast, when a significant number of photons are emitted in the cryogenic environment of a superconducting hadron collider, it is necessary to introduce a liner between the cold vacuum wall and the beam. This necessity was recognized even before the LHC design, as the result of experiments performed to study the photon stimulated desorption conditions expected at cryogenic temperatures in the SSC. More refined experiments have been performed during the LHC design process.

The basic mechanism of gas desorption at the beam pipe or liner surface is electron excitation from the incident photons. These photons have enough energy to eject or excite even the inner shell electrons. There are several unwanted electron relaxation results at surfaces in the VLHC like ejected and secondary electrons, neutrals, and ion desorption. The "redistribution" of (mostly) hydrogen molecules continually enhances the background vacuum pressure in both the LHC and the VLHC, reducing the beam life time. Hydrogen molecules are physisorbed at the cold walls with a binding energy of less than $1 \mathrm{eV}$. The dependence of the hydrogen vapor pressure on the wall temperature is shown in Fig. 59.

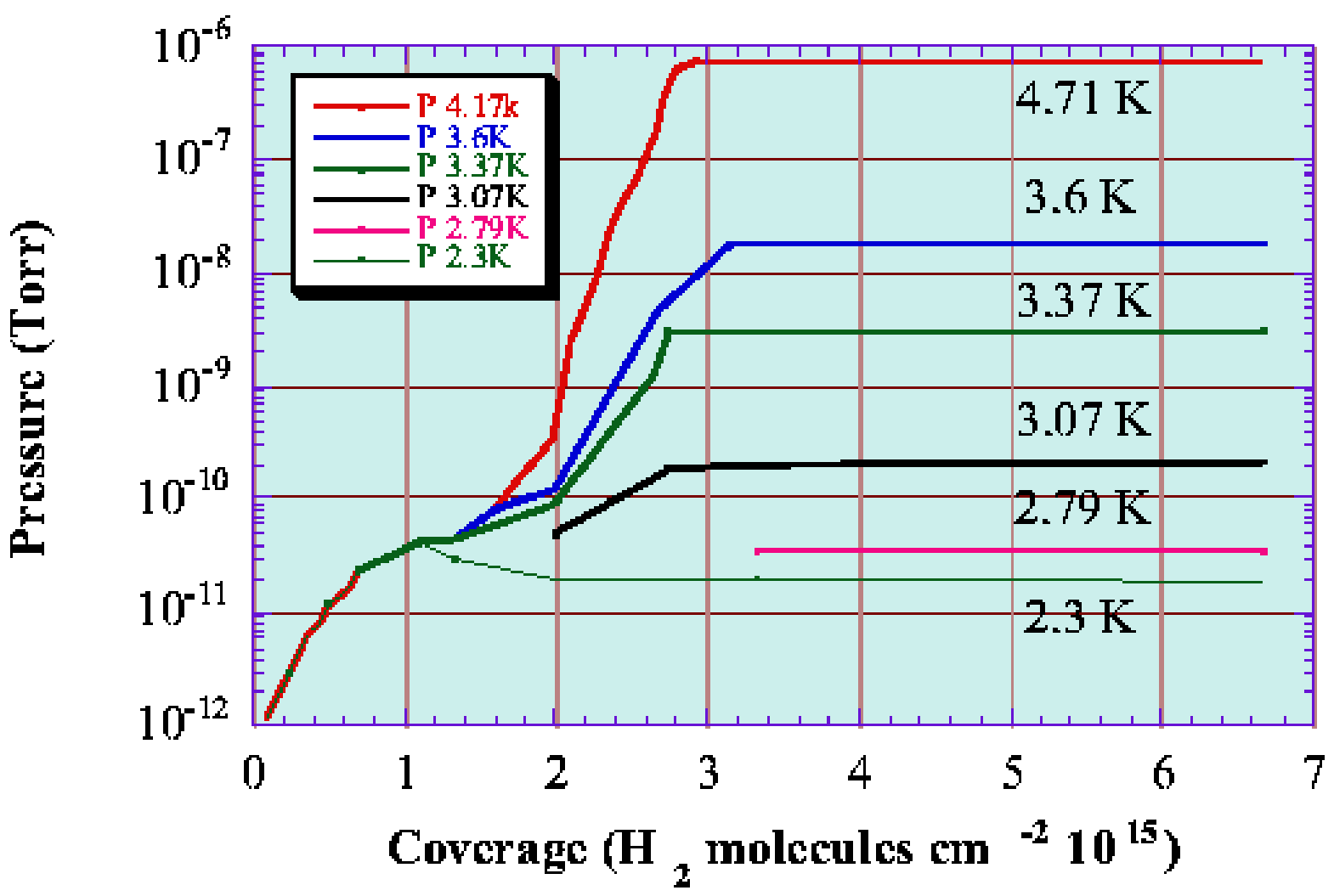

\section{Benvemuti, R. Calder}

Figure 59: Hydrogen vapor pressure as a function of coverage and temperature.

The LHC is confronted with the real possibility of an electron cloud instability. When free electrons are present in the beam pipe, the electric field of the passing proton bunches can accelerate them to energies in the keV range. They then hit the other side of the liner wall, creating secondary electrons which may be accelerated by a following bunch. An avalanche process can occur, leading to a large thermal beam load and even to beam loss. One important parameter, the secondary electron yield, 


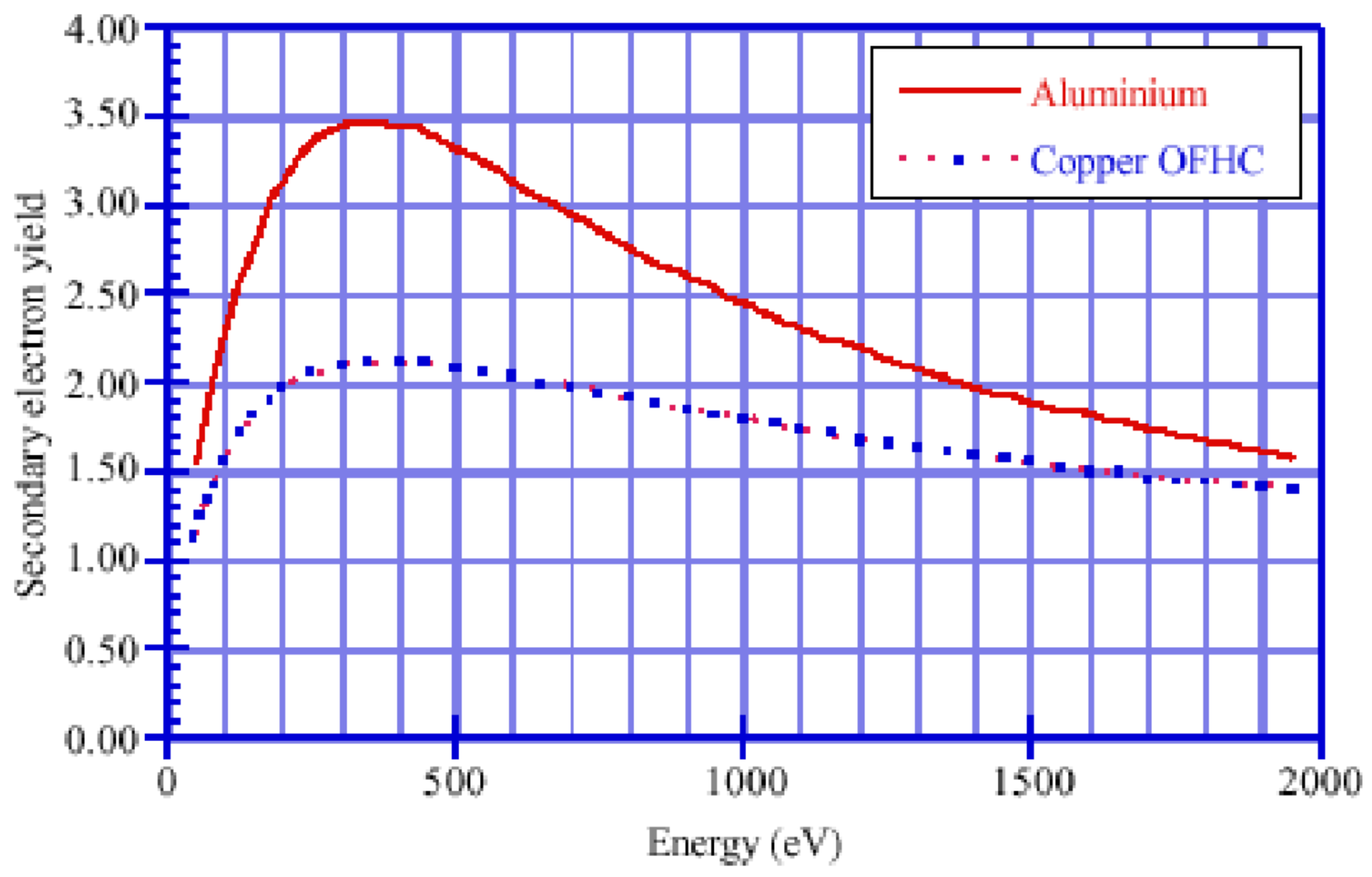

Figure 60: Yield of secondary electron as a function of energy of primary electrons.

determines the average number of secondary electrons per incident electron. It varies with the wall materials, surface conditions, and with the incident electron energy, as shown in Fig. 60. Another important parameter is the spacing between bunches. If the time between bunches is much larger than the typical time for electrons to travel between the two liner walls, then the avalanche process may be suppressed. The photon reflectivity of the liner or vacuum wall is another important parameter.

A dedicated VLHC workshop, Synchrotron Radiation Effects in the VLHC, was organized in September 2000, at Brookhaven National Laboratory, to discuss the operational impact of synchrotron radiation in both low and high field rings. Major conclusions from the workshop include:

1. A liner is necessary in both low and high field rings

2. Even with a liner, a significant pump-out time will be necessary to reach stable operating vacuum conditions. The hydrogen vapor pressure in the beam pipe must less than $\sim 10^{-10}$ Torr for beam lifetimes larger than 100 hours. The coverage of hydrogen molecules, and the vapor pressure, rise rapidly above cold bore temperatures of $3 \mathrm{~K}$. If the cold bore temperature is larger than $3 \mathrm{~K}$, then additional pump-out getter materials between the bore and the liner might be required.

3. Preliminary calculations show that the electron cloud instability could be serious if the number of protons per bunch is larger than $2 \times 10^{10}$. Fortunately this is more than a factor of 2 larger than the nominal population of $N=9 \times 10^{9}$.

4. The power load from the synchrotron radiation to the cryogenic system can be reduced if it is possible to place a special "warm" photon stopper once every few dipoles. 


\subsection{Electron Cloud Instability}

The most critical issue is secondary electron (SE) multipacting. The secondary electron induced heat load and beam instabilities are not expected to be problems at beam intensities below the multipacting threshold. Above the threshold, some approach has to be taken to suppress electron multipacting.

The recent SPS machine experiment is a good reference for electron cloud build-up in accelerators like the VLHC. The SPS observed electrons cloud starting to build up after the passage of 30 bunches, at single bunch intensities larger than $N \geq 6 \times 10^{10}$ with a bunch spacing of $25 \mathrm{~ns}$ between bunches of full length $4 \mathrm{~ns}$. No electron build up was observed for $N=2.5 \times 10^{11}$, with a bunch spacing of $130 \mathrm{~ns}$ between bunches of full length $7 \mathrm{~ns}$.

The two key elements in a first estimate of the electron cloud build-up threshold are bunch spacing, and bunch intensity. The bunch spacing determines the fraction of secondary electrons that survive until the next bunch passage, and is therefore a critical factor in electron cloud build-up. The general condition for build-up is $\alpha \bar{Y}>1$, where $\alpha$ is the secondary electron survival fraction, and $\bar{Y}$ is the average SE yield after the bunch passage.

The secondary electrons have an energy distribution [55]

$$
\frac{d N_{e}}{d E}=\sqrt{\frac{E}{E_{s m}}} \frac{N_{e}}{\sqrt{2 \pi} E_{s m}} \exp \left(-\frac{1}{2} \frac{E}{E_{s m}}\right)
$$

where $N_{e}$ is the total number of electrons, and $E_{s m}$ is the energy at the peak of the distribution, with $E_{s m} \approx 2.5 \mathrm{eV}$ for a stainless steel vacuum chamber. The secondary electrons also have an angular distribution, given by the cosine of the angle to the normal of the metal surface, independent of the projectile incident angle [56]. The secondary electron transit time distribution $d N_{e} / d t$ can be calculated from the SE energy and angular distributions. Finally, the secondary electron survival fraction is obtained as a function of the length of the beam gap,

$$
\alpha(t)=1-\int_{0}^{t}\left(\frac{d N_{e}}{d t}\right) d t
$$

Figure 61 shows the secondary electron survival fraction as a function of bunch gap for a high field beam pipe liner with a radius of $10 \mathrm{~mm}$. Also shown is the SPS survival fraction, where the $22.5 \mathrm{~mm} \times 70 \mathrm{~mm}$ chamber is represented by an average radius of $30 \mathrm{~mm}$. More than $90 \%$ of the secondary electrons survive with the VLHC bunch gap of $18.8 \mathrm{~ns}$. This alone does not necessarily indicate electron cloud build-up, but it does motivate a close look at bunch intensity effects.

Consider two bunch intensity aspects, the average kinetic energy gained by secondary electrons as the next bunch passes, and the beam potential well. The average kinetic energy gain is independent of the bunch length, but depends on the bunch intensity, the transverse size of the beam, and the beam chamber size. The beam potential well depends on all of these factors, including the bunch length. The average energy gain, which can be used for a simplified estimate of the electron cloud build-up, is $[55,56]$

$$
\langle\Delta E\rangle=\frac{e}{2 m_{e}}\left(\frac{N e Z_{0}}{2 \pi b}\right)^{2} \ln \left(\frac{b}{a}\right)
$$

where $m_{e}$ is the mass of electron, $N$ is the proton bunch population, $Z_{0}=377 \Omega$, and $b$ and $a$ are the radii of the chamber and the beam, respectively. According to the Seiler model [57] the yield is then

$$
Y=1.11 \delta_{\max }\left(\frac{E}{E_{p m}}\right)^{-0.35}\left(1-\exp \left(-2.3\left(\frac{E}{E_{p m}}\right)^{1.35}\right)\right)
$$

where $E_{p m}$ is the projectile (electron) energy that gives rise to maximum yield, $\delta_{\max }$.

These parameters are in the ranges $1.2 \leq \delta_{\max } \leq 1.6$ and $300 \mathrm{eV} \leq E_{p m} \leq 500 \mathrm{eV}$, for stainless steel without the scraping effect. Therefore, we may take $\delta_{\max }=1.4$, and $E_{p m}=400 \mathrm{eV}$, without losing generality. Figure 62 shows a typical secondary electron yield versus the primary electron energy, for a stainless steel surface. Only primary electrons with a kinetic energy from $150 \mathrm{eV}$ to $1400 \mathrm{eV}$ have a yield larger than unity.

For the SPS, with a bunch intensity of $N \geq 6 \times 10^{10}$, using the vertical chamber half aperture of $b_{v}=22.5 \mathrm{~mm}$ and $a=3.2$ $\mathrm{mm}$, the average secondary electron energy gain is $\langle\Delta E\rangle=112 \mathrm{eV}$. For the VLHC high field ring, with $N=0.9 \times 10^{10}, b=10$ $\mathrm{mm}$, and $a=0.074 \mathrm{~mm}$ at collision, the average gain is $\langle\Delta E\rangle=32 \mathrm{eV}$.

A yield greater than unity is obtained in the VLHC high field ring only for average energy gains above a threshold, attained above a bunch intensity threshold given by $N \geq 3.15 \times 10^{10}$. When the effect of finite bunch spacing is included, through which the VLHC survival fraction is lower than that in the SPS, the VLHC intensity threshold becomes even larger. 


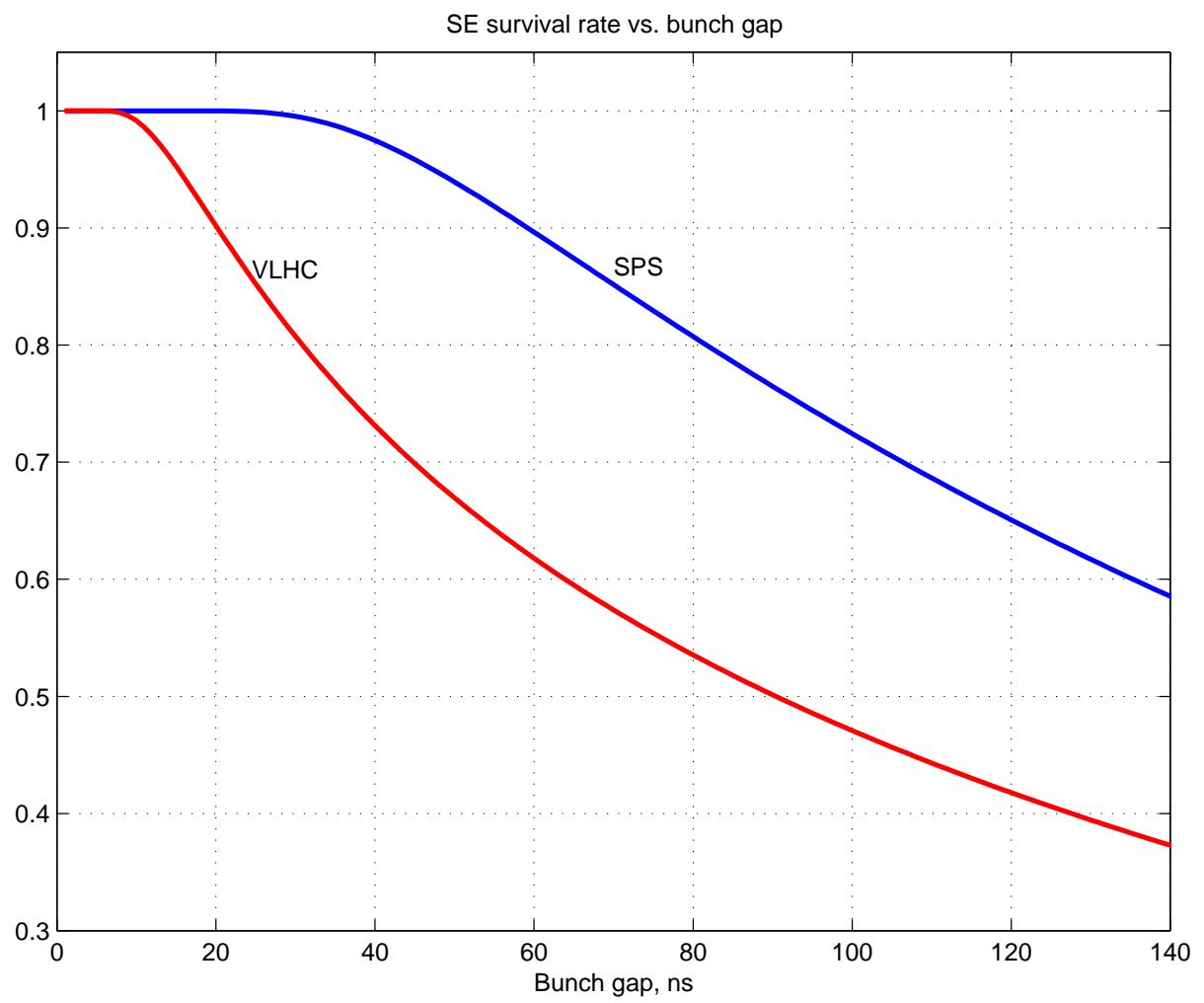

Figure 61: Secondary electron survival fraction as a function of bunch gap, for the high field ring and for the SPS.

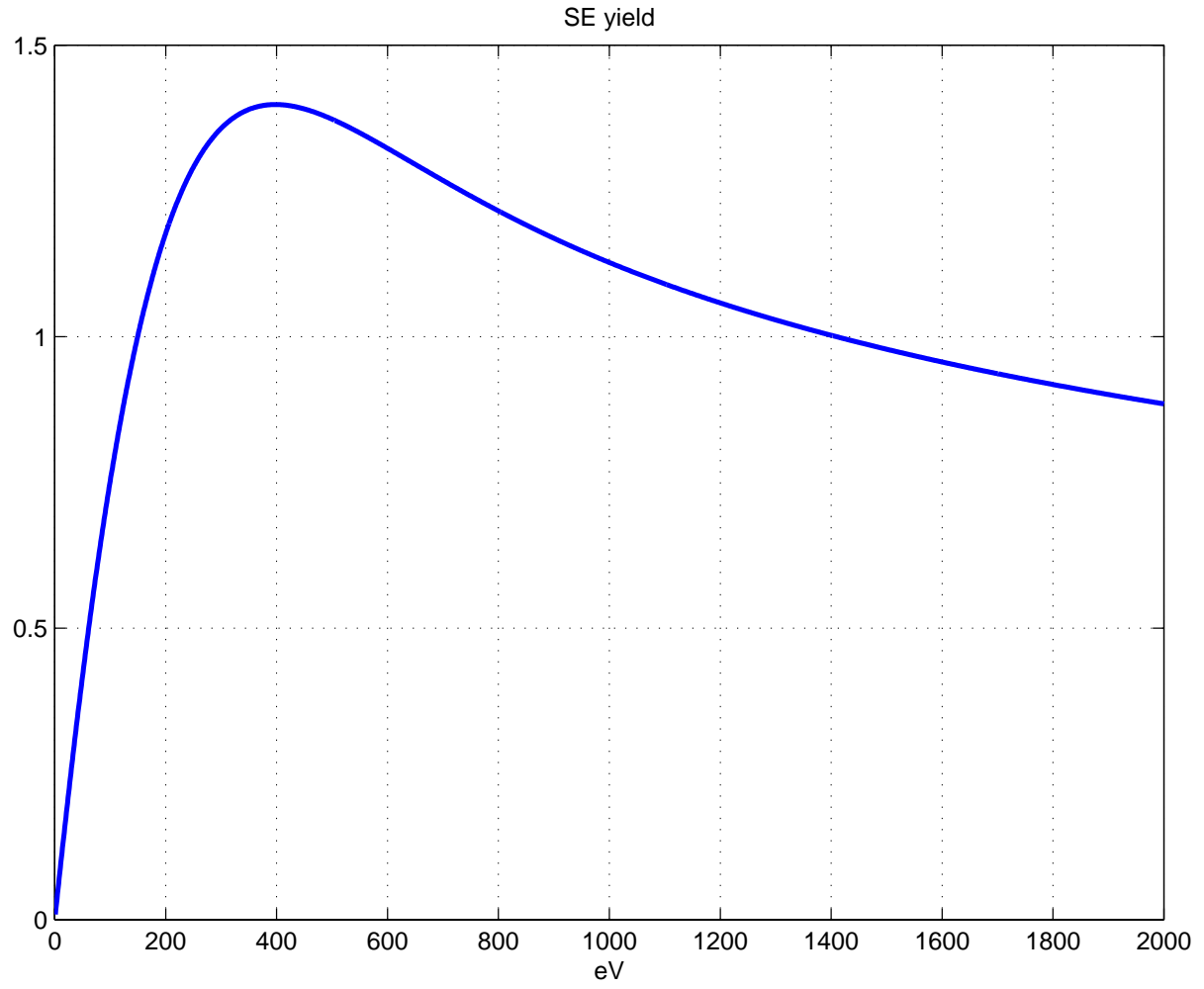

Figure 62: Secondary electron yield vs. primary electron energy, stainless steel surface. 
The distribution of the secondary electron energy gain is very different for long and short bunches [58]. This is because bunches with same intensity but different lengths have different potential wells. It is especially relevant that the bunch in the SPS experiment was more than 10 times longer than the VLHC bunch at collision. The beam potential well is

$$
V_{p o t}=\frac{e \lambda}{2 \pi \epsilon_{0}}\left(0.5+\ln \left(\frac{b}{a}\right)-\frac{r^{2}}{2 a^{2}}\right)
$$

where $r$ is the radial position, $\epsilon_{0}$ is the permittivity in free space, and $\lambda$ is the beam line density. The VLHC beam potential well at collision is shown on the left in Figure 63, while the SPS is shown on the right. The SPS bunch intensity is much higher than the VLHC, yet the VLHC beam potential is much deeper. A reliable approach using the beam potential well to determine the electron cloud threshold has yet to be developed.
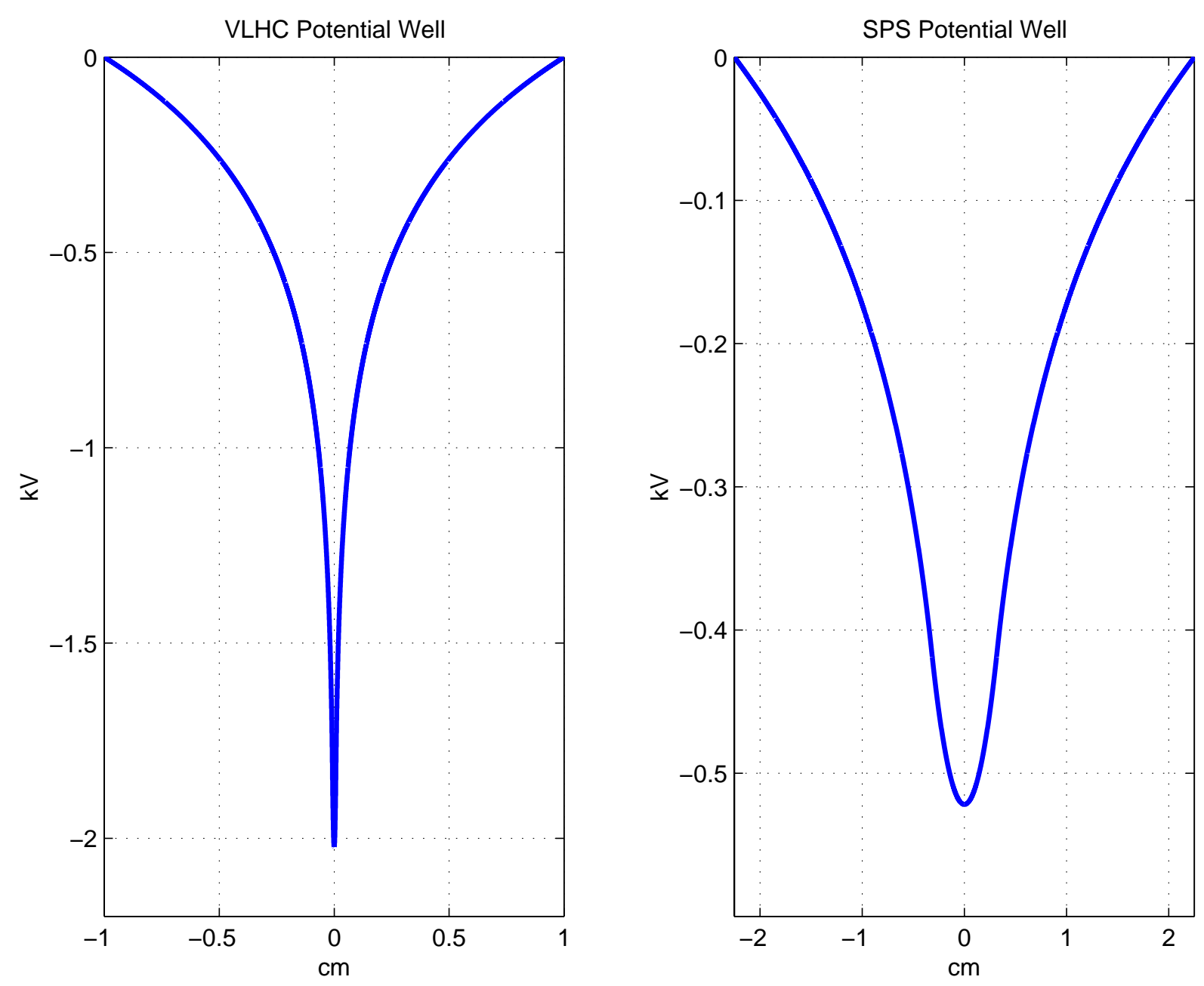

Figure 63: The beam potential well in the VLHC at collision (left), and in the SPS at injection (right).

Table 33 lists electron cloud parameters for four hadron colliders. The three key parameters are the bunch spacing $t_{b s}$, the average energy gain $\langle\Delta E\rangle$, and the beam potential well depth $V_{p o t}$. In summary, the bunch spacing of the VLHC is slightly less than in the SPS, but the chamber size is also smaller, and so the secondary electron survival fraction is smaller in the VLHC than in the SPS. The average kinetic energy gained by secondary electrons at each bunch passage is much smaller in the high field VLHC than the SPS threshold, and the chance of electron cloud in the VLHC is therefore expected to be small. The beam potential of the VLHC is comparable with the SPS, but much less severe than the LHC. Further study is needed to ensure the complete safety of the high field ring from electron cloud problems. 


\begin{tabular}{|lcccc|}
\hline & $\begin{array}{c}\text { SPS } \\
\text { e-c/No }\end{array}$ & SSC & $\begin{array}{c}\text { LHC } \\
\text { Inject/Store }\end{array}$ & $\begin{array}{c}\text { VLHC } \\
\text { Inject/Store }\end{array}$ \\
& & & & \\
$R(\mathrm{~m})$ & 1100 & 13201 & 4243 & 37089 \\
$E_{k}(\mathrm{TeV})$ & 0.026 & 20 & $0.45 / 7$ & $10 / 87.5$ \\
$f_{0}(\mathrm{kHz})$ & 43.4 & 3.62 & 11.25 & 1.287 \\
$N\left(10^{10}\right)$ & $6 / 25$ & 0.735 & 10 & 0.9 \\
$\lambda\left(10^{10} / \mathrm{m}\right)$ & $7.4 / 21.4$ & 3.8 & $29.1 / 49.5$ & $4.0 / 13.0$ \\
$b(\mathrm{~mm})$ & 22.5 & 16 & 17.4 & 10 \\
$a(\mathrm{~mm})$ & 3.2 & 0.126 & $1.15 / 0.293$ & $0.21 / 0.074$ \\
$\sigma_{\ell}(\mathrm{cm})$ & $30 / 53$ & 7.3 & $13 / 7.5$ & $8.2 / 2.6$ \\
$\sigma_{\tau}(\mathrm{ns})$ & $1.0 / 1.75$ & 0.24 & $0.43 / 0.25$ & $0.273 / 0.087$ \\
$t_{b s}(\mathrm{~ns})$ & $\mathbf{2 5} / \mathbf{1 3 0}$ & $\mathbf{1 6}$ & $\mathbf{2 5}$ & $\mathbf{1 8 . 8}$ \\
$\langle\Delta E\rangle(\mathrm{eV})$ & $\mathbf{1 1 2 / 1 9 5 2}$ & $\mathbf{8 . 3}$ & $\mathbf{7 2 7 / 1 0 9 3}$ & $\mathbf{2 3 . 6 / 3 1 . 1}$ \\
$V_{p o t}(\mathrm{kV})$ & $\mathbf{0 . 5 2 / 1 . 5 1}$ & $\mathbf{0 . 5 8}$ & $\mathbf{2 . 7 / 6 . 5}$ & $\mathbf{0 . 5 0 / 2 . 0}$ \\
& & & & \\
\hline
\end{tabular}

Table 33: Comparison of key electron cloud parameters between four proton colliders.

\subsection{Energy Deposition at the Interaction Region}

In a collider with beam energies $E_{\text {beam }}$ of $87.5 \mathrm{TeV}$ and a peak luminosity $\mathcal{L}$ of $2 \times 10^{34} \mathrm{~cm}^{-2} \mathrm{~s}^{-1}$ energy deposition from the particles created in pp collisions is significant and could eventually pose a problem for the superconducting magnets in the interaction region. The deposited energy on one side of the IR, $E_{d}$, in units of $\mathrm{kW}$ is given by:

$$
E_{d}=N \times 0.5 \times E_{f w d}
$$

where $\mathrm{N}$ corresponds to the number of collisions per hour and $E_{f w d}$ to the total energy of particles in forward direction in units of $\mathrm{kWh}$. In general, $\mathrm{N}$ is determined by:

$$
N=\sigma_{\text {tot }} \times \mathcal{L} \times 3600 .
$$

Table 34 shows a list of the dominant processes and their cross sections at $\sqrt{s}=175000 \mathrm{GeV}$ according to the high energy event generator PYTHIA [59]. While the first 2 processes do not contribute exclusively to energy deposition in forward direction, the latter four do, accumulating to a "forward" cross section of $66 \mathrm{mb}$.

The elastic scattering and diffraction cross sections are reflected in the pattern in fig. 64 depicting high energy tracks only at small polar angles $\Theta$. In fact, PYTHIA predicts about $40 \%$ of all collisions with basically all energy, i.e. more or less the incoming protons, distributed in forward direction.

These events are of particular interest for us in cases when they have scattering angles smaller than the apex angle of the D1 magnets $30 \mathrm{~m}$ downstream of the IR. Under this condition the charged collision products are bent in the dipole field of the D1 magnets and contribute eventually to the energy deposition in the down stream IR quadrupoles Q1A and Q1B. Fig. 65 shows the energy distribution of charged and uncharged tracks with a polar angle $\Theta<\Theta$ apex with $\Theta_{\text {apex }}=0.00042$ rad. These tracks belong to an overall sample of 1000 generated PYTHIA events. The continuum of the charged particle energy distribution (lower left plot in fig 65) has a mean of $76360 \mathrm{GeV}$. The distribution peaks at $2 \times E_{\text {beam }}$.

The approximate number of collision particles hitting the IR dipoles and quadrupoles can be estimated by neglecting the uncharged particles, which aren't affected by the magnetic field of the D1 dipoles, but taking into account particles with small angles emerging from subprocesses 1 and 2 in Tab. 34. Assuming a mean total energy of $76 \mathrm{TeV}$ of all charged particles contained within the beam pipe for $75 \%$ of the collisions and about $175 \mathrm{TeV}$ for the remaining $25 \%$ an upper limit of $24 \mathrm{~kW}$ energy deposition on either side of the IR can be derived. However, this energy deposition is spread over a certain distance since the point where the particles hit the wall of the beam pipe depends on both, incoming angle and particle energy. For effective collimation or any other counter measure this has to be studied with more detail taking into account the machine lattice and magnet transfer functions. 


\begin{tabular}{|clc|}
\hline index & subprocess & $\sigma(\mathrm{mb})$ \\
& & \\
1 & $f+g->f+g$ & 20.9 \\
2 & $g+g->g+g$ & 63.2 \\
3 & Elastic scattering & 35.9 \\
4 & Single diffractive (XB) & 8.2 \\
5 & Single diffractive (AX) & 8.2 \\
6 & Double diffractive & 13.7 \\
\hline
\end{tabular}

Table 34: Cross sections of the dominant processes at $175.0 \mathrm{TeV}$ collisions (PYTHIA). $f$ and $g$ correspond to fermion and gluon, respectively.

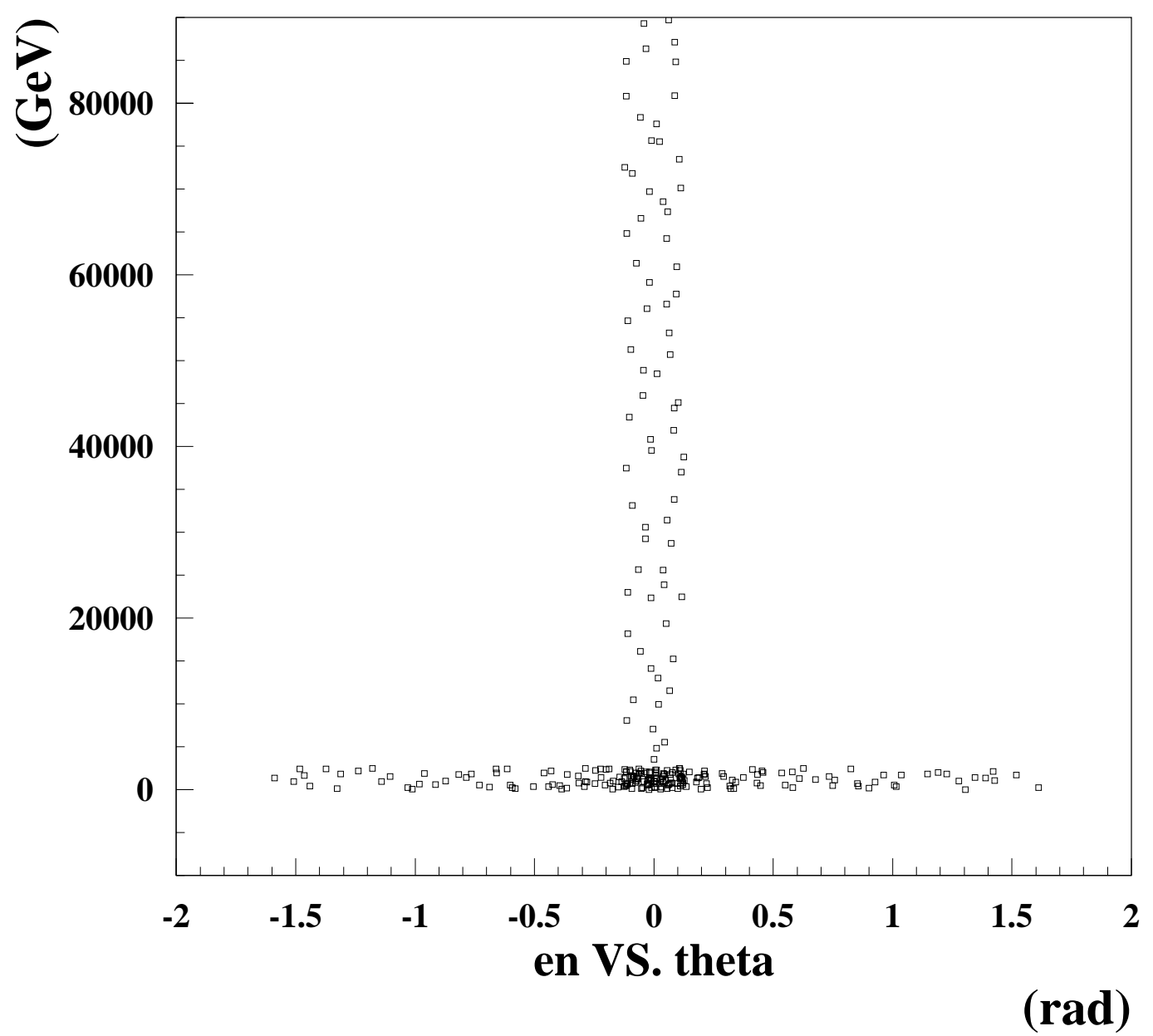

Figure 64: The energy of stable final state particles as a function of polar angle $\Theta$ (PYTHIA). 


\section{forward energy distributions}

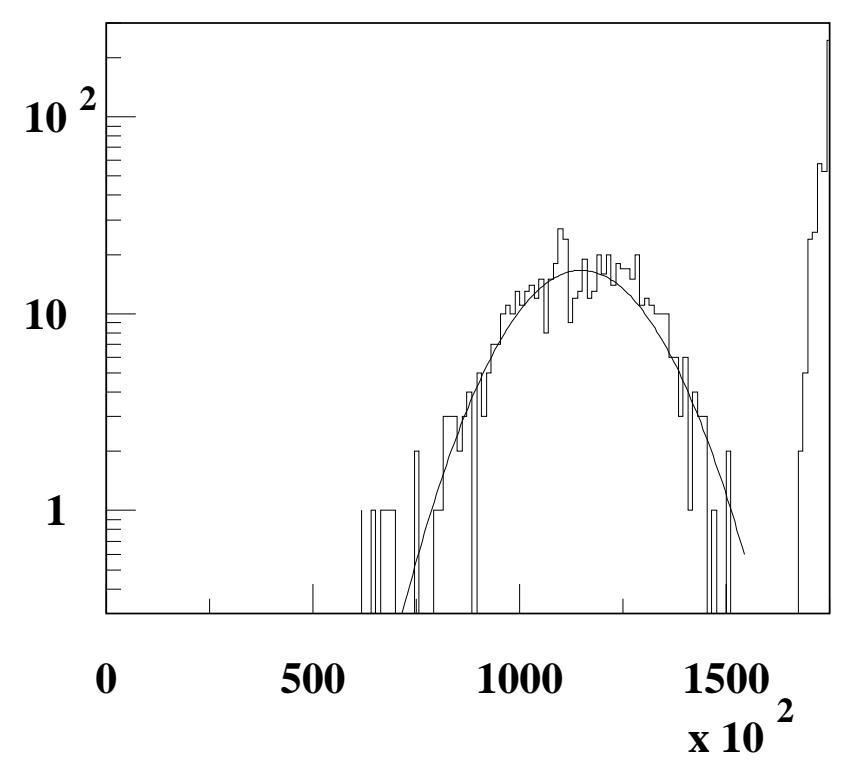

all particles $(\mathrm{GeV})$

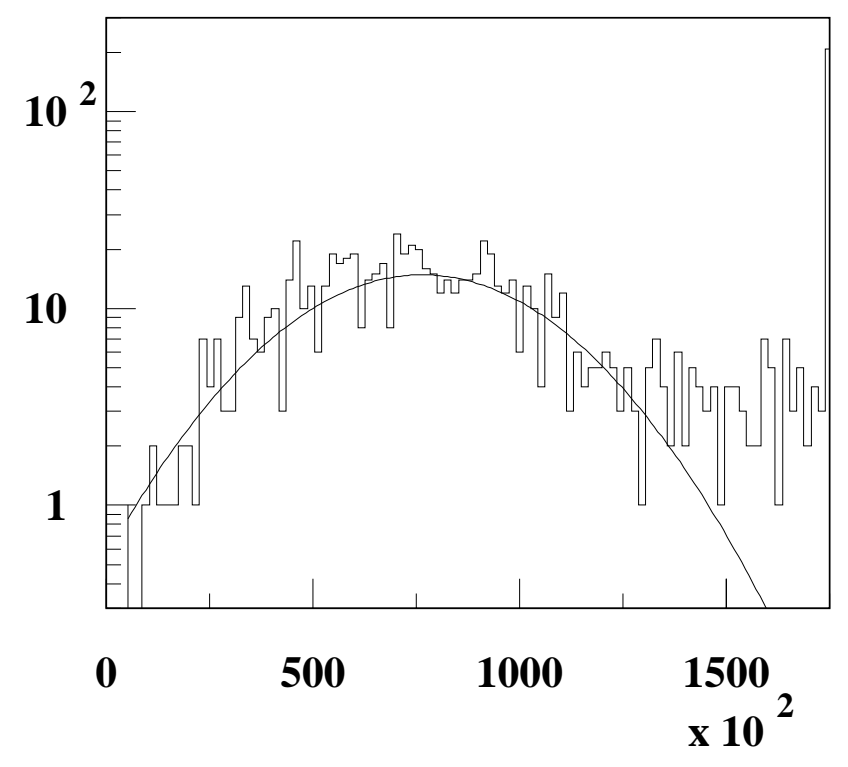

charged particles $(\mathrm{GeV})$

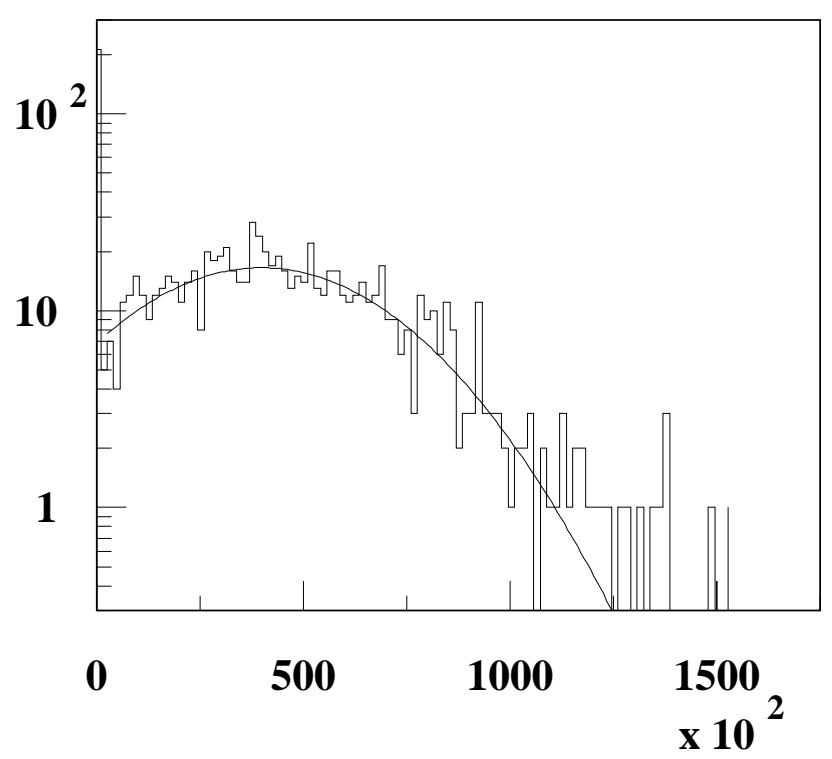

uncharged particles $(\mathrm{GeV})$

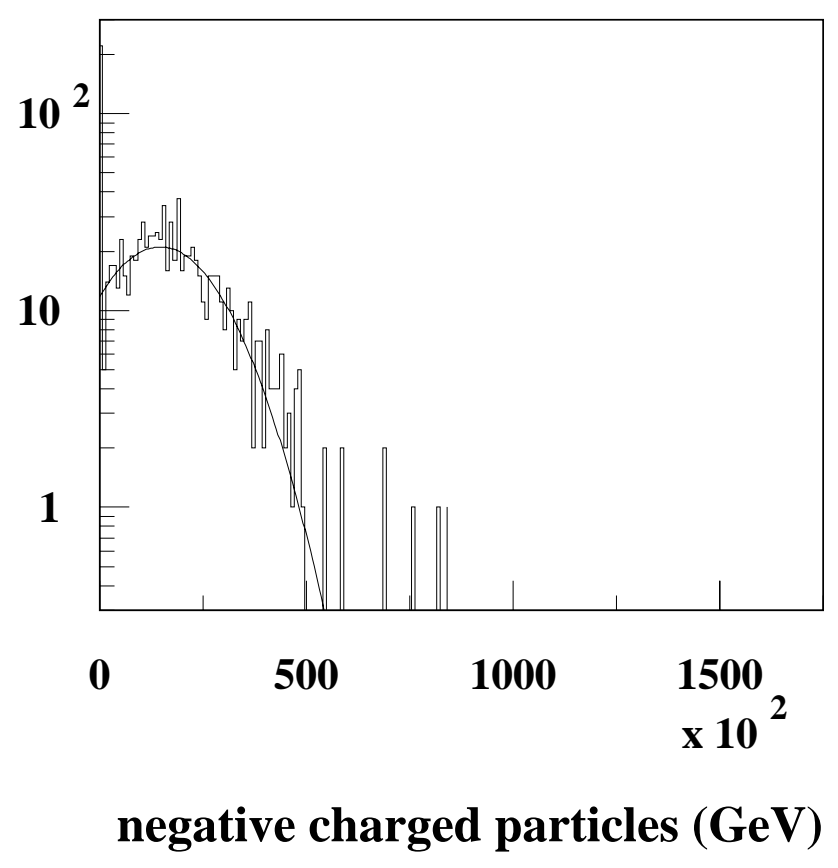

Figure 65: Energy distribution of charged and uncharged tracks in forward direction in collisions at $\sqrt{s}=175000 \mathrm{GeV}$ (PYTHIA). 


\subsection{Beam Transfer from Low Field to High Field}

A conceptual design for transferring the beam of the low field VLHC (LFVLHC) to the high field VLHC (HFVLHC) has been devised. A beam energy of $20 \mathrm{TeV}$ has been used, while a lower beam energy (10 TeV, say) would easily be accommodated. The transfers will take place between long straight sections, 6 cells in length, in each machine. Since there are two beams in the LFVLHC, obviously two transfer lines are needed, one for each of the beams. The transfer line is based on the design of the straight section in the LFVLHC. In the LFVLHC the value of $\beta_{x}$ at the end of the straight sections for one beam is equal to the value of $\beta_{y}$ for the other beam. In the HFVLHC, on the other hand, the two beams have the same values for the lattice functions at the ends of the straight section. Thus, the design for two transfer lines will be different. Table 35 gives the values of the lattice functions at the upstream end of the transfer line for the beam in the low field VLHC and the downstream end for the high field VLHC.

\begin{tabular}{|ccccc|}
\hline & $\begin{array}{c}\text { Initial Value } \\
\text { Beam 1 }\end{array}$ & $\begin{array}{c}\text { Final Value } \\
\text { Beam 1 }\end{array}$ & $\begin{array}{c}\text { Initial Value } \\
\text { Beam 2 }\end{array}$ & $\begin{array}{c}\text { Final Value } \\
\text { Beam 2 }\end{array}$ \\
$\beta_{x}$ & 411.671 & 459.60 & 86.781 & 459.6 \\
$\alpha_{x}$ & 0.0 & 0.0 & 0.0 & 0.0 \\
$\beta_{y}$ & 86.781 & 79.89 & 411.671 & 79.89 \\
$\alpha_{y}$ & 0.0 & 0.0 & 0.0 & 0.0 \\
$\eta_{x}$ & 0.0 & 0.0 & 0.0 & 0.0 \\
$\eta_{x}^{\prime}$ & 0.0 & 0.0 & 0.0 & 0.0 \\
$\eta_{y}$ & 0.0 & 0.0 & 0.0 & 0.0 \\
$\eta_{x}^{\prime}$ & 0.0 & 0.0 & 0.0 & 0.0 \\
\hline
\end{tabular}

Table 35: Values for the lattice functions at the ends of the transfer line

\section{Bends}

The LFVLHC and the HFVLHC are assumed to have elevations differing by $1 \mathrm{~m}$. Further it is assumed that the beams in each accelerator are offset horizontally by $0.3 \mathrm{~m}^{3}$ The concept in the design of the transfer line is to bend the beam vertically in the first half cell so that it clears the next quadrupole in the straight section. The rest of the transfer line is then between the two accelerators until we enter the high field VLHC with another strong bend. Because the required displacements for the two transfer lines are the same, identical bends were chosen for the two lines. In the design of the straight section cells for the LFVLHC, between the quadrupoles, there are approximately $88 \mathrm{~m}$ in each half cell for bend magnets. In order to simplify the design further, the bends are reflected about the midpoint of the transfer line. The characteristics of the bends are shown in Table 36. Except for the first (and last) bends the required fields are small. Even for the first and last bends the fields are only $\approx 1.7 \mathrm{~T}$. The strengths of the bend magnets in the other cells are found, as mentioned earlier, by reversing the field in the corresponding magnet found by reflecting about the midpoint of the transfer line. Figure 66 shows the space-projections of the transfer line trajectory.

\section{Quadrupoles}

In order to match the computed lattice functions, including the dispersion, at the end of the straight sections, with the lattice functions of the high field VLHC, the strengths of the quadrupoles were adjusted. A good match was found and the quality of the solutions is shown in Table 37. The computed values of the quadrupole strengths for the two transfer lines are given in Table 38. Figures 67 and 68 show the values of the lattice functions for these solutions. In both cases the maximum value of $\beta$, which is a primary design consideration, is reasonable. The dispersion functions $\eta$ and $\eta^{\prime}$ also are well behaved and well matched.

\footnotetext{
${ }^{3}$ The two beams are vertically separated in the high field ring, and horizontally separated in the low field ring. The $1 \mathrm{~m}$ vertical displacement represents the average vertical displacement of the two rings. These vertical and horizontal displacements are subject to change.
} 


\begin{tabular}{|ccccc|}
\hline Location & \multicolumn{2}{c}{ Horizontal Bend } & \multicolumn{2}{c|}{ Vertical Bend } \\
Cell & Length $(\mathrm{m})$ & Field $(\mathrm{T})$ & Length $(\mathrm{m})$ & Field $(\mathrm{T})$ \\
& & & & \\
$1 \mathrm{a}$ & - & - & 88.88542 & -1.67748 \\
$1 \mathrm{~b}$ & - & - & 88.88542 & 1.70864 \\
$2 \mathrm{a}$ & 45.0 & -0.53752 & 25.0 & -1.4175 \\
$2 \mathrm{~b}$ & 45.0 & -0.30042 & - & - \\
$3 \mathrm{a}$ & 35.0 & 0.77493 & - & - \\
$3 \mathrm{~b}$ & 35.0 & 0.30230 & 35.0 & 0.9338 \\
\hline
\end{tabular}

Table 36: Characteristics of the bending magnets, at $20 \mathrm{TeV}$.

\begin{tabular}{|ccccc|}
\hline & $\begin{array}{c}\text { Desired Value } \\
\text { Beam 1 }\end{array}$ & $\begin{array}{c}\text { Fitted Value } \\
\text { Beam 1 }\end{array}$ & $\begin{array}{c}\text { Desired Value } \\
\text { Beam 2 }\end{array}$ & $\begin{array}{c}\text { Fitted Value } \\
\text { Beam 2 }\end{array}$ \\
$\beta_{x}$ & 459.60 & 459.604 & 459.6 & 459.591 \\
$\alpha_{x}$ & 0.000 & 0.000 & 0.000 & 0.000 \\
$\beta_{y}$ & 79.89 & 79.903 & 79.89 & 79.978 \\
$\alpha_{y}$ & 0.000 & 0.000 & 0.000 & 0.000 \\
$\eta_{x}$ & 0.000 & 0.000 & 0.000 & 0.000 \\
$\eta_{x}^{\prime}$ & 0.000 & 0.000 & 0.000 & 0.000 \\
$\eta_{y}$ & 0.000 & -0.001 & 0.000 & 0.000 \\
$\eta_{x}^{\prime}$ & 0.000 & 0.000 & 0.000 & 0.003 \\
\hline
\end{tabular}

Table 37: Goal and fitted values of the transfer line lattice functions.

\section{Summary Remarks}

The design of the transfer lines does not appear to be a major problem at this stage of the planning for a VLHC. A problem may develop in injecting the beam into the high field VLHC depending on the physical dimensions of the quadrupoles in the straight sections. If that turns out to be the case it may be desirable to have a long section without the quadrupoles. The current design could be replaced with a Collins Straight Section or a Utility Straight Section depending on which is more convenient. 
Floor coordinates. to design the transier line from the if to the hf machine.SURVEY

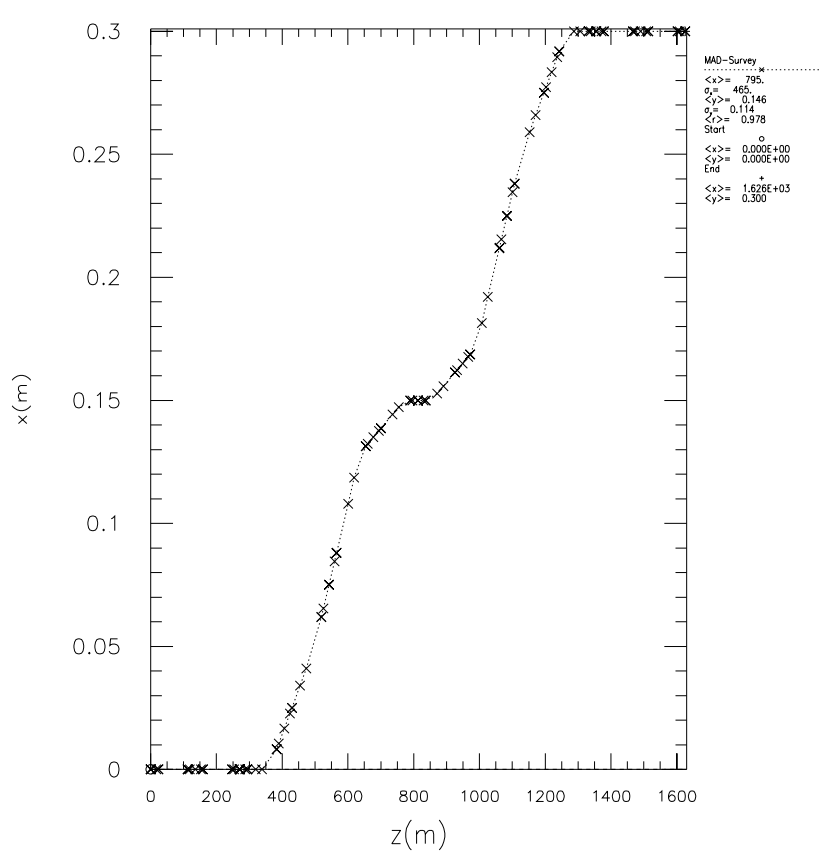

Floor coordinates.

to design the transfer line from the It to the hi machine.SURVEY

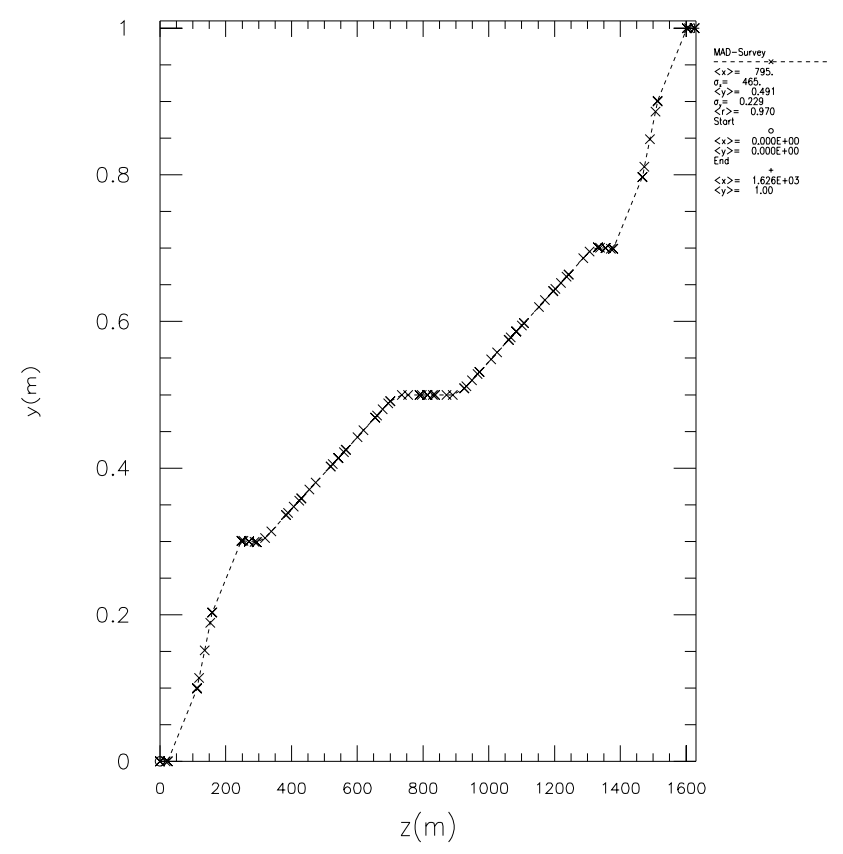

Floor coordinates.

to design the transfer line from the if to the hif machine.SURVEY

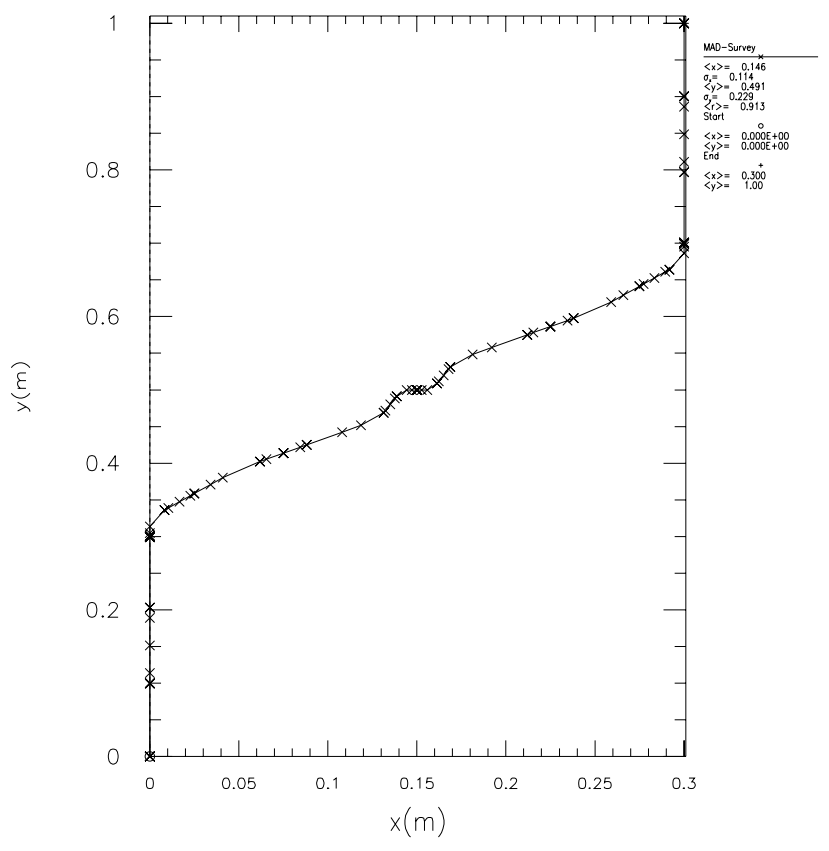

Figure 66: Trajectory of the $10 \mathrm{TeV}$ transfer line from Stage 1 to Stage 2 rings. 


\begin{tabular}{|cccc|}
\hline Element & Length (m) & $\begin{array}{c}\text { Beam 1 } \\
\text { Strength (T/m) }\end{array}$ & $\begin{array}{c}\text { Beam 2 } \\
\text { Strength (T/m) }\end{array}$ \\
qf1a & 6.096 & 69.478 & 57.530 \\
qd1a & 6.096 & -68.749 & 28.861 \\
qf1b & 6.096 & 72.901 & -103.223 \\
qd1b & 6.096 & -67.943 & -73.489 \\
qf2a & 6.096 & 68.610 & 77.402 \\
qd2a & 6.096 & -69.970 & 66.445 \\
qf2b & 6.096 & 67.790 & 8.382 \\
qd2b & 6.096 & -69.277 & -63.330 \\
qf3a & 6.096 & 71.666 & 74.037 \\
qd3a & 6.096 & -68.748 & -65.333 \\
qf3b & 6.096 & 72.637 & 90.471 \\
qd3b & 6.096 & -68.983 & -65.984 \\
qf4a & 6.096 & 69.209 & 82.759 \\
qd4a & 6.096 & -67.605 & -64.176 \\
qf4b & 6.096 & 68.744 & 83.447 \\
qd4b & 6.096 & -69.424 & -76.139 \\
qf5a & 6.096 & 72.168 & 116.861 \\
qd5a & 6.096 & -71.358 & -78.787 \\
qf5b & 6.096 & 73.118 & 31.044 \\
qd5b & 6.096 & -69.720 & -80.102 \\
qf6a & 6.096 & 70.145 & 91.717 \\
qd6a & 6.096 & -68.856 & -81.655 \\
qf6b & 6.096 & 67.919 & 51.517 \\
qd6b & 6.096 & -70.698 & -86.018 \\
\hline
\end{tabular}

Table 38: Quadrupole strengths in the transfer line, at $20 \mathrm{TeV}$. 
$\beta_{x}, \beta_{y}$ vs Distance.

$\beta_{x}, \beta_{y}$ vs Distance.

to design the tronsfer line from the If to the ht mochine.TWISS

vhc-r-design.mod

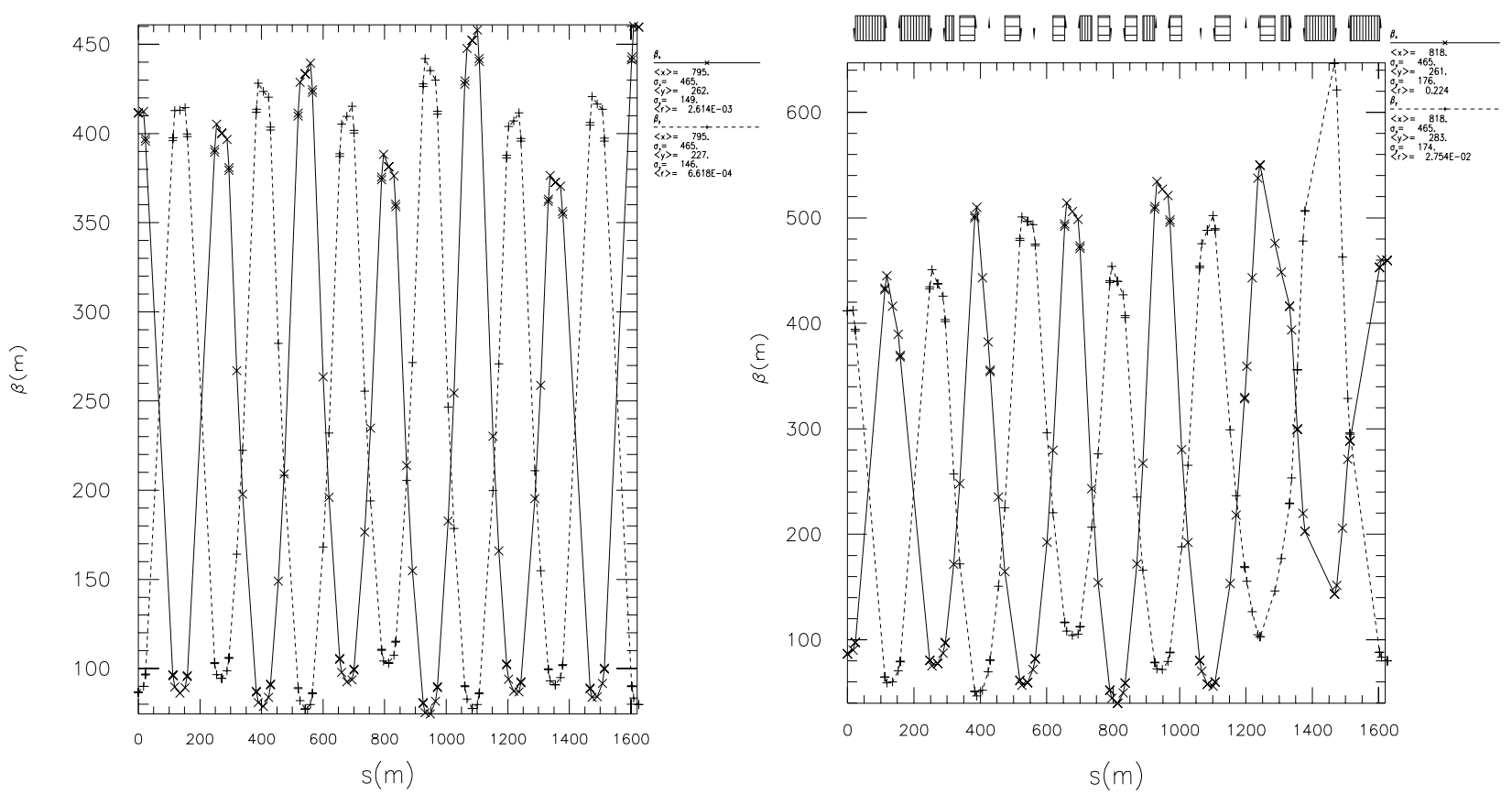

Figure 67: Lattice beta functions for the $10 \mathrm{TeV}$ transfer lines.
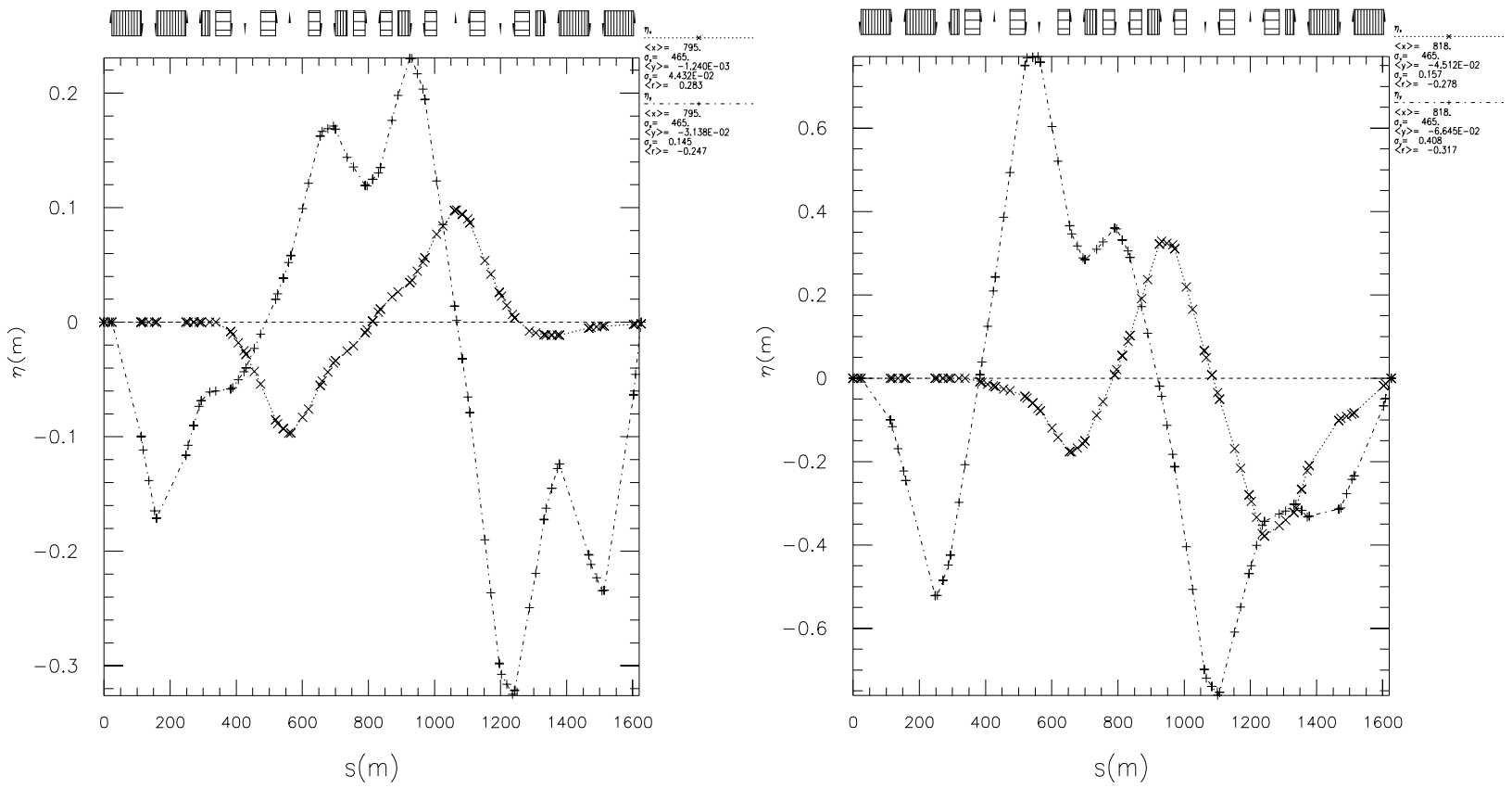

Figure 68: Dispersion functions for the $10 \mathrm{TeV}$ transfer lines. 


\subsection{Fundamental Problems, and Future Research Fronts}

Four major issues could severely alter HF ring performance estimates, given our present knowledge of Accelerator Physics and the preliminary state of the HF design:

1. Energy deposition. The power of debris products exiting the collision point goes far beyond current experience. Is there an acceptable engineering solution?

2. Operational aperture. The physical beam sizes are so small that the operational aperture is probably more important than the dynamic aperture. How far can the closed orbit move before the beam is lost? Is operational feedback necessary on closed orbits, tunes, and chromaticities in order to accelerate beam to top energy?

3. Instabilities. Although the HF ring is in general an order of magnitude more immune to instability issues than the LF ring (due to its higher rigidity), there is still room for considerable concern. For example, the electron cloud heat load in the cryogenic beam environment could be unacceptable.

4. Diffusion. The operational scenario assumes that the beam emittances decrease by an order of magnitude or more, with a damping time of order 2.5 hours ( $10^{7}$ turns). Our basic understanding of slow diffusion mechanisms does not preclude the possibility that this is fundamentally impossible. Can we reliably extrapolate from other colliders?

In addition to these major issues - which may not be soluble on a short timescale - there are many others which need to be addressed, some of which are amenable to shorter term resolution. It is convenient to group all issues, major and minor, short and long term, into research fronts, as follows:

1. Diffusion. Evaluate more accurately the effects of ground motion, modulational diffusion, Intra Beam Scattering, BeamBeam induced diffusion, et cetera.

2. Lattice design. Develop realistic optics for the interaction region (doublet and triplet optics), the LF to HF transfer line, vertical dispersion suppression, beam abort, and collimation regions. Evaluate the optimum crossing angles and planes, and the optimum half cell length. Much of this needs to be done in close collaboration with LF lattice design efforts.

3. Simulation. Particle tracking studies to evaluate magnet field quality tables. Energy deposition simulations of the IR debris products. Dynamic optics, et cetera, in store evolution simulations. Test the conjecture that the operational aperture (measured in $\mathrm{mm}$ ) is more critical than the dynamic aperture (measured in beam sigmas). Develop specifications for operational feedback on closed orbits, tunes, and chromaticities. Evaluate arc and IR correction schemes.

4. Instabilities. Estimate the strength of resistive wall, electron cloud, and other relevant instabilities [60]. Evaluate the need for collective effect feedback systems. Develop an impedance budget and estimate.

5. Energy scaling. Determine the practical limits to maximum luminosity, such as cryogenic power load, IR energy deposition, number of events per crossing, and capital or operating costs. Evaluate how the maximum luminosity permitted by these constraints varies with beam energy, et cetera.

6. Beam experiments. Develop a plan for beam based experiments at existing hadron colliders, performed by a formal multi-laboratory collaboration. Major experimental sub-topics might include

- single particle dynamics

- diffusion

- feedback (operational and collective) 


\section{Parameters}

\subsection{Low Field Parameter Tables}

\begin{tabular}{|lrl|}
\hline & 233.037 & $\mathrm{~km}$ \\
Circumference, $C$ & 35.0 & $\mathrm{~km}$ \\
Average arc radius, $R$ & 2 & \\
Number of interaction points & 135.486 & $\mathrm{~m}$ \\
Half cell length, $L_{h c}$ & 3.875 & $\mathrm{mrad}$ \\
Half cell bend angle, $\theta_{h c}$ & 1720 & \\
Half cell count & 24 & \\
Half cell harmonic, $n_{\lambda}$ & 5.645 & $\mathrm{~m}$ \\
Bunch spacing $(53.1 \mathrm{MHz}), S_{B}$ & 18.8 & $\mathrm{~ns}$ \\
& 41280 & \\
Number of buckets & 90.0 & $\mathrm{deg}$ \\
Phase advance per cell & 1.286 & $\mathrm{kHz}$ \\
Revolution frequency & 0.778 & $\mathrm{~ms}$ \\
Revolution period, $T$ & 371520 & \\
Harmonic number, $h$ & 478.0 & $\mathrm{MHz}$ \\
RF frequency $(9 \times 53.1)$ & & \\
& & \\
\hline
\end{tabular}

Table 39: Fundamental lattice parameters common to both low and high field rings.

\begin{tabular}{|llrl|}
\hline & ALIAS & $L / L_{h c}$ & COMMENT \\
DOsulE & & & \\
Injection/Abort straight & DS & 3 & \\
Dispersion Module & DM8 & 8 & contains 2 arc half cells \\
Interaction Region & IR & 12 & \\
Dispersion Module & DM & 6 & \\
Crossing "straight" & X & 2 & \\
Dispersion Module & DM & 6 & \\
Interaction Region & IR & 12 & \\
Dispersion Module & DM8 & 8 & contains 2 arc half cells \\
Injection/Abort straight & IA & 10 & \\
Dispersion Suppressor & DS & 3 & \\
Utility total & & & \\
Arc & & 80 & \\
GRAND TOTAL & & 780 & \\
& & 1720 & 2 arcs plus 2 utilities \\
\hline
\end{tabular}

Table 40: Sequence of optical modules in the on-site utility region of the VLHC. 
Storage energy, $E$

Peak luminosity, $L_{\max }$

Inelastic cross section

Total cross section

Collision debris power, per IP

Dipole field at storage

Distance from IP to first magnet

Injection energy

Fill time

Acceleration time

Synchrotron radiation damping time, $T_{0}$

Energy loss per turn, $U_{0}$

Initial transverse emittance $(\mathrm{H}, \mathrm{V})$

Number of bunches, $M$

Fraction of buckets filled

Collision beta (horz, vert), $\beta^{*}$

Initial bunch intensity, $N$

Protons per beam

Beam current

Synch. rad. power, per beam $P$

Magnet linear heat load

Stored energy, per beam $U$
$20 \mathrm{TeV}$

$10^{34} \mathrm{~cm}^{-2} \mathrm{~s}^{-1}$

100 mbarn

130 mbarn

$3 \mathrm{~kW}$

$2.0 \mathrm{~T}$

$21 \mathrm{~m}$

$0.9 \mathrm{TeV}$

$60 \mathrm{~min}$

$1000 \mathrm{~s}$

$100 \mathrm{~h}$

$38 \mathrm{keV}$

$1.5 \mu \mathrm{m}$

37152

$90 \%$

$0.3 \mathrm{~m}$

$2.610^{10}$

$9.510^{14}$

$195 \mathrm{~mA}$

$7.4 \mathrm{~kW}$

$0.07 \mathrm{~W} / \mathrm{m}$

3.0 GJ

Table 41: Nominal parameters for stores in the low field ring.

\begin{tabular}{|lrl|}
\hline & & \\
INJECTION & & \\
Emittance, rms (H and V) & 1.5 & $\mu \mathrm{m}$ \\
Momentum width, rms & 607 & $10^{-6}$ \\
Arc bunch size (betatron + dispersion) & 1.24 & $\mathrm{~mm}$ \\
RF voltage & 50.0 & $\mathrm{MV}$ \\
Longitudinal rms emittance & 0.4 & $\mathrm{eV}-\mathrm{s}$ \\
Bunch length, rms & 30 & $\mathrm{~mm}$ \\
Synchrotron frequency & 13.1 & $\mathrm{~Hz}$ \\
& & \\
STORE, BEGINNING & & \\
Emittance, rms (H and V) & 1.5 & $\mu \mathrm{m}$ \\
Momentum width, rms & 62 & $10^{-6}$ \\
Arc bunch size (betatron + dispersion) & 0.196 & $\mathrm{~mm}$ \\
Full crossing angle & 153 & $\mu \mathrm{rad}$ \\
RF voltage & 50 & $\mathrm{MV}$ \\
Longitudinal rms emittance & 0.4 & $\mathrm{eV}-\mathrm{s}$ \\
Bunch length, rms & 38 & $\mathrm{~mm}$ \\
Synchrotron tune & .00245 & \\
Synchrotron frequency & 3.16 & $\mathrm{~Hz}$ \\
& & \\
Beam-beam parameter & .002 & \\
IP bunch size (H,V) & 4.6 & $\mu \mathrm{m}$ \\
& & \\
\hline
\end{tabular}

Table 42: Store parameters for LF ring, including longitudinal and RF. 


\begin{tabular}{|lrl|}
\hline & 218.419 & \\
Horizontal tune & 218.425 & \\
Vertical tune & 192.1 & \\
Transition gamma & 411 & $\mathrm{~m}$ \\
Maximum arc beta & 1.56 & $\mathrm{~m}$ \\
Maximum arc dispersion & 0.3 & $\mathrm{~m}$ \\
Horz, Vert $\beta^{*}$ (store) & 0.56 & $\mathrm{~km}$ \\
Maximum $\widehat{\beta}$, injection & 11.27 & $\mathrm{~km}$ \\
Maximum $\widehat{\beta}$, store & & \\
& & \\
\hline
\end{tabular}

Table 43: Short list of some low field lattice parameters.

\begin{tabular}{|lrl|}
\hline & & \\
DIPOLE & 0.09 & $\mathrm{~T}$ \\
Field at injection & 2.0 & $\mathrm{~T}$ \\
Field at store & 9.0 & $\mathrm{~T} / \mathrm{m}$ \\
Bend Magnet field gradient, store & 32.897 & $\mathrm{~km}$ \\
Bend radius & & \\
& & \\
ARC CELLS & 24 & \\
Half cell harmonic & 135.486 & $\mathrm{~m}$ \\
Half cell length & 3.875 & $\mathrm{mrad}$ \\
Half cell bend angle & 1568 & \\
Half cell count & 2 & \\
Bend Magnets per half cell & 3242.667 & \\
Bend Magnets, total (equiv.) & 65.75 & $\mathrm{~m}$ \\
Bend magnetic length & 97 & $\%$ \\
Bend Magnet fill factor (half cell) & & \\
& & \\
DISPERSION SUPPRESSOR CELLS & 18 & \\
Half cell harmonic & 101.614 & $\mathrm{~m}$ \\
Half cell length & 2.583 & $\mathrm{mrad}$ \\
Half cell bend angle & 80 & \\
Half cell count & 2 & \\
Dipoles per half cell & & \\
&
\end{tabular}

Table 44: Arc and dispersion suppressor low field magnet and cell parameters.

\begin{tabular}{|lcccc|}
\hline Magnet & Gradient $[\mathrm{T} / \mathrm{m}]$ & Aperture $[\mathrm{mm}]$ & Length [m] & Type \\
Q1 & 302 & 85 & 10.9 & 1-in-1 \\
Q2a,b & 304 & 85 & 9.22 & 1-in-1 \\
Q3 & 302 & 85 & 10.9 & 1 -in-1 \\
Q4 & 51 & 22 & 12.19 & 2 -in-1 \\
Q5a,b & 69 & 22 & 12.19 & 2 -in-1 \\
Q6a,b & 62 & 22 & 12.19 & 2 -in-1 \\
Q7 & 0.8 & 22 & 7.62 & 2 -in-1 \\
& & & & \\
\hline
\end{tabular}

Table 45: Design parameters of the low field interaction region magnets. 


\begin{tabular}{|lrrl|}
\hline & & & \\
RMS ERROR & ARC & IR & \\
QUADRUPOLES & & & \\
Transverse offset & 0.25 & 0.2 & $\mathrm{~mm}$ \\
BPM to quad offset & 0.15 & 0.1 & $\mathrm{~mm}$ \\
Roll & 0.5 & 0.5 & $\mathrm{mrad}$ \\
Field Error $\Delta K / K$ & 0.5 & 0.1 & $10^{-3}$ \\
& & & \\
DIPOLES & & & \\
Roll & 0.5 & & $\mathrm{mrad}$ \\
Field Error $\Delta B / B$ & 1 & & $10^{-4}$ \\
& & & \\
\hline
\end{tabular}

Table 46: Tolerance summary for magnet alignment

$\begin{array}{lrll}\text { CORRECTORS } & & \\ \text { Dipole magnetic length } & 0.5 & \mathrm{~m} \\ \text { Dipole max strength } & 0.5 & \text { T m } \\ \text { Quadrupole magnetic length } & 0.5 & \mathrm{~m} \\ \text { Quadrupole max strength } & 25 & \mathrm{~T} / \mathrm{m} \\ \text { Sextupole magnetic length } & 0.8 & \mathrm{~m} \\ \text { Sextupole max strength } & 1750 & \mathrm{~T} / \mathrm{m}^{2}\end{array}$

Table 47: Corrector magnetic lengths and strengths, low field 


\subsection{High Field Parameter Tables}

\begin{tabular}{|lrl|}
\hline & 233.037 & $\mathrm{~km}$ \\
Circumference, $C$ & 35.0 & $\mathrm{~km}$ \\
Average arc radius, $R$ & 2 & \\
Number of interaction points & 135.486 & $\mathrm{~m}$ \\
Half cell length, $L_{h c}$ & 3.875 & $\mathrm{mrad}$ \\
Half cell bend angle, $\theta_{h c}$ & 1720 & \\
Half cell count & 24 & \\
Half cell harmonic, $n_{\lambda}$ & 5.645 & $\mathrm{~m}$ \\
Bunch spacing $(53.1 \mathrm{MHz}), S_{B}$ & 18.8 & $\mathrm{~ns}$ \\
& 41280 & \\
Number of buckets & 90.0 & $\mathrm{deg}$ \\
Phase advance per cell & 1.286 & $\mathrm{kHz}$ \\
Revolution frequency & .778 & $\mathrm{~ms}$ \\
Revolution period, $T$ & 371520 & \\
Harmonic number, $h$ & 478.0 & $\mathrm{MHz}$ \\
RF frequency $(9 \times 53.1)$ & & \\
& & \\
\hline
\end{tabular}

Table 48: Fundamental lattice parameters common to both low and high field rings.

\begin{tabular}{|llrl|}
\hline & ALIAS & $L / L_{h c}$ & COMMENT \\
MODULE & & & \\
Dispersion Suppressor & DS & 3 & \\
Dispection/Abort straight & IA & 10 & \\
Interaction Mogion & DM8 & 8 & contains 2 arc half cells \\
Dispersion Module & IR & 12 & \\
Crossing "straight" & X & 6 & \\
Dispersion Module & DM & 2 & \\
Interaction Region & IR & 12 & \\
Dispersion Module & DM8 & 8 & contains 2 arc half cells \\
Injection/Abort straight & IA & 10 & \\
Dispersion Suppressor & DS & 3 & \\
& & & \\
Utility total & & 80 & \\
Arc & & 780 & \\
GRAND TOTAL & & 1720 & 2 arcs plus 2 utilities \\
& & & \\
\hline
\end{tabular}

Table 49: Sequence of optical modules in the on-site cluster region of the VLHC. 
Storage energy, $E$

Peak luminosity, $L_{\max }$

Inelastic cross section

Total cross section

Collision debris power, per IP

Dipole field at storage

Distance from IP to first magnet

Injection energy

Fill time

Acceleration time

Synchrotron radiation damping time, $T_{0}$

Energy loss per turn, $U_{0}$

Natural transverse emittance $(\mathrm{H})$

Natural RMS momentum width

Number of bunches, $M$

Fraction of buckets filled

Collision beta horz, $\beta_{x}^{*}$

Collision beta vert, $\beta_{y}^{*}$

Equilibrium emittance ratio, $\kappa$

Initial bunch intensity, $N$

Protons per beam

Beam current

Synch. rad. power, per beam $P$

Dipole linear heat load

Stored energy, per beam $U$
87.5 TeV

$2 \times 10^{34} \mathrm{~cm}^{-2} \mathrm{~s}^{-1}$

130 mbarn

169 mbarn

$73 \mathrm{~kW}$

$9.765 \mathrm{~T}$

$30 \mathrm{~m}$

$10 \mathrm{TeV}$

$30 \mathrm{~s}$

2000 s

$2.48 \mathrm{~h}$

$15.3 \mathrm{MeV}$

$.0397 \mu \mathrm{m}$

$5.510^{-6}$

37152

$90 \%$

$3.7 \mathrm{~m}$

$0.37 \mathrm{~m}$

0.1

$\begin{array}{ll}7.5 & 10^{9}\end{array}$

$2.79 \quad 10^{14}$

$57.4 \mathrm{~mA}$

$.88 \mathrm{MW}$

$4.7 \mathrm{~W} / \mathrm{m}$

3.9 GJ

Table 50: Nominal parameters for stores in the high field ring. 


\begin{tabular}{|lrl|}
\hline INJECTION & & \\
Emittance, rms (H and V) & 1.5 & $\mu \mathrm{m}$ \\
Momentum width, rms & 233.1 & $10^{-6}$ \\
Arc bunch size (betatron) & 254 & $\mu \mathrm{m}$ \\
Arc bunch size (dispersion) & 330 & $\mu \mathrm{m}$ \\
RF voltage & 50.0 & $\mathrm{MV}$ \\
Longitudinal rms emittance & 2.0 & $\mathrm{eV}-\mathrm{s}$ \\
Longitudinal beta & 351 & $\mathrm{~m}$ \\
Bunch length, rms & 81.9 & $\mathrm{~mm}$ \\
Synchrotron tune & 00280 & \\
Synchrotron frequency & 3.60 & $\mathrm{~Hz}$ \\
& & \\
STORE BEGINNING & & \\
Emittance, rms (H and V) & 1.5 & $\mu \mathrm{m}$ \\
Momentum width, rms & 64.8 & $10^{-6}$ \\
Arc bunch size (betatron) & 86 & $\mu \mathrm{m}$ \\
Arc bunch size (dispersion) & 92.0 & $\mu \mathrm{m}$ \\
Full crossing angle & 30.6 & $\mu \mathrm{rad}$ \\
RF voltage & 200 & $\mathrm{MV}$ \\
Longitudinal rms emittance & 2.0 & $\mathrm{eV}-\mathrm{s}$ \\
Longitudinal beta & 520 & $\mathrm{~m}$ \\
Bunch length, rms & 33.7 & $\mathrm{~mm}$ \\
Synchrotron tune & .00189 & \\
Synchrotron frequency & 2.44 & $\mathrm{~Hz}$ \\
& & \\
EARLY PLATEAU (Flat beams) & & \\
Beam-beam parameter & .008 & \\
Emittance, rms (H) & .161 & $\mu \mathrm{m}$ \\
Emittance, rms (V) & .016 & $\mu \mathrm{m}$ \\
Momentum width, rms & 50.0 & $10^{-6}$ \\
Arc bunch size (betatron) & 28.2 & $\mu \mathrm{m}$ \\
Arc bunch size (dispersion) & 71.0 & $\mu \mathrm{m}$ \\
IP bunch size (H) & 2.53 & $\mu \mathrm{m}$ \\
IP bunch size (V) & .25 & $\mu \mathrm{m}$ \\
Full crossing angle & 10.0 & $\mu \mathrm{rad}$ \\
RF voltage & 200 & $\mathrm{MV}$ \\
Longitudinal rms emittance & 1.191 & $\mathrm{eV}-\mathrm{s}$ \\
Longitudinal beta & 520 & $\mathrm{~m}$ \\
Bunch length, rms & 26.0 & $\mathrm{~mm}$ \\
Synchrotron tune & .00189 & \\
Synchrotron frequency & 2.44 & $\mathrm{~Hz}$ \\
& & \\
\hline
\end{tabular}

Table 51: High field store parameters, including longitudinal and RF. 


\begin{tabular}{|lcc|}
\hline & FLAT & ROUND \\
& & \\
Flatness parameter, $\kappa$ & 0.1 & 1 \\
Beam-beam parameter $\xi_{x}=\xi_{y}$ & .008 & .008 \\
Peak luminosity $L\left(10^{34} \mathrm{~cm}^{-2} \mathrm{~s}^{-1}\right)$ & 2.0 & 2.0 \\
& & \\
Average luminosity, $20 \mathrm{hr} L_{\text {ave }}\left(10^{34} \mathrm{~cm}^{-2} \mathrm{~s}^{-1}\right)$ & 1.02 & 0.98 \\
Initial bunch intensity $N\left(10^{9}\right)$ & 7.5 & 7.5 \\
Collision beta horz $\beta_{x}^{*}(\mathrm{~m})$ & 3.7 & 0.71 \\
Collision beta vert $\beta_{y}^{*}(\mathrm{~m})$ & 0.37 & 0.71 \\
Maximum beta horz $\widehat{\beta_{x}}(\mathrm{~km})$ & 7.84 & 14.58 \\
Maximum beta vert $\widehat{\beta_{y}}(\mathrm{~km})$ & 10.75 & 14.58 \\
Horizontal emittance $\epsilon_{x}(\mu \mathrm{m})$ & .161 & .082 \\
Vertical emittance $\epsilon_{y}(\mu \mathrm{m})$ & .016 & .082 \\
Collision beam size horz $\sigma_{x}^{*}(\mu \mathrm{m})$ & 2.53 & 0.79 \\
Collision beam size vert $\sigma_{y}^{*}(\mu \mathrm{m})$ & 0.25 & 0.79 \\
Maximum beam size horz $\widehat{\sigma_{x}}(\mu \mathrm{m})$ & 116 & 113 \\
Maximum beam size vert $\widehat{\sigma}{ }_{y}(\mu \mathrm{m})$ & 43 & 113 \\
Angular beam size horz $\sigma_{x}^{\prime}(\mu \mathrm{r})$ & 0.68 & 1.11 \\
Angular beam size vert $\sigma_{y}^{\prime}(\mu \mathrm{r})$ & 0.68 & 1.11 \\
& & \\
Total crossing angle $\alpha(\mu \mathrm{r})$ & 10.0 & 10.0 \\
Separation distance, $L_{\text {sep }}(\mathrm{m})$ & 30 & 120 \\
Number of long range collisions per IR & 20 & 84 \\
Long range tune shift per IR, horz $\left|\Delta Q_{x}\right|$ & .0008 & .0166 \\
Long range tune shift per IR, vert $\left|\Delta Q_{y}\right|$ & .0081 & .0166 \\
& & \\
\hline
\end{tabular}

Table 52: Flat and round beam performance parameters, quoted after about 6 hours at the "early plateau" just after peak luminosity, when the horizontal and vertical beam-beam parameters are both saturated.

\begin{tabular}{|lrl|}
\hline & 218.19 & \\
Horizontal tune & 212.18 & \\
Vertical tune & 194.13 & \\
Transition gamma & 26.53 & $10^{-6}$ \\
Slip factor & 459 & $\mathrm{~m}$ \\
Maximum arc beta & 1.42 & $\mathrm{~m}$ \\
Maximum arc dispersion & 33.36 & $10^{4} \mathrm{Tm}$ \\
Rigidity at injection & 291.9 & $10^{3} \mathrm{Tm}$ \\
Rigidity at store & 0.37 & $\mathrm{~m}$ \\
Vertical $\beta^{*}$ (store) & 3.7 & $\mathrm{~m}$ \\
Horizontal $\beta^{*}$ (store) & .61 & $\mathrm{~km}$ \\
Maximum $\widehat{\beta}$, injection & 7.84 & $\mathrm{~km}$ \\
Maximum Horz $\widehat{\beta}$, store & 10.75 & $\mathrm{~km}$ \\
Maximum Vert $\widehat{\beta}$, store & & \\
\hline
\end{tabular}

Table 53: Short list of some high field lattice parameters. 


$\begin{array}{lrl} & & \\ \text { DIPOLE } & 1.116 & \mathrm{~T} \\ \text { Field at injection } & 9.765 & \mathrm{~T} \\ \text { Field at store } & 29.887 & \mathrm{~km} \\ \text { Bend radius } & 40 & \mathrm{~mm} \\ \text { Coil full width } & 20 & \mathrm{~mm} \\ \text { Liner full width } & .29 & \mathrm{~m} \\ \text { Vertical bore separation } & 828 & \mathrm{~kJ} / \mathrm{m} \\ \text { Stored energy (2 bores) } & & \\ & & \\ \text { ARC CELLS } & 24 & \\ \text { Half cell harmonic } & 135.486 & \mathrm{~m} \\ \text { Half cell length } & 3.875 & \mathrm{mrad} \\ \text { Half cell bend angle } & 1568 & \\ \text { Half cell count } & 7 & \\ \text { Dipoles per half cell } & 10976 & \\ \text { Dipole count, total } & 16.546 & \mathrm{~m} \\ \text { Dipole magnetic length } & 85.5 & \% \\ \text { Dipole fill factor } & 8.066 & \mathrm{~m} \\ \text { Quad magnetic length } & 385.4 & \mathrm{~T} / \mathrm{m} \\ \text { Quad field gradient } & & \\ & & \\ \text { DISPERSION SUPPRESSOR CELLS } & 18 & \\ \text { Half cell harmonic } & 101.614 & \mathrm{~m} \\ \text { Half cell length } & 2.583 & \mathrm{mrad} \\ \text { Half cell bend angle } & 80 & \\ \text { Half cell count } & 5 & \\ \text { Dipoles per half cell } & 400 & \\ \text { Dipole count, total } & 15.443 & \mathrm{~m} \\ \text { Dipole magnetic length } & 76.0 & \% \\ \text { Dipole fill factor } & 10.775 & \mathrm{~m} \\ \text { Quad magnetic length } & 288.7 & \mathrm{~T} / \mathrm{m} \\ \text { Quad field gradient (QD1) } & 376.2 & \mathrm{~T} / \mathrm{m} \\ \text { Quad field gradient (QF2) } & 281.9 & \mathrm{~T} / \mathrm{m} \\ \text { Quad field gradient (QD3) } & 390.2 & \mathrm{~T} / \mathrm{m} \\ \text { Quad field gradient (QF4) } & & \\ & & \\ & & \\ & & \\ & & \\ & & \\ & & \\ & & \\ & & \\ & & \end{array}$

Table 54: Arc and dispersion suppressor magnet and cell parameters.

\begin{tabular}{|lccccc|}
\hline Magnet & Field [T] & Gradient [T/m] & Aperture [mm] & Length [m] & Type \\
D1A & 16 & & & & \\
D1B & 12 & & 50 & 12.1 & 1-in-1 \\
D2 & 12 & & 50 & 6.0 & 1 -in-1 \\
Q1A & & 400 & 30 & 11.1 & 2 -in-1 \\
Q1B & & 600 & 30 & 12.4 & 2 -in-1 \\
Q2A & & 600 & 30 & 7.9 & 2 -in-1 \\
Q2B & & 600 & 30 & 7.9 & 2-in-1 \\
& & & & & \\
\hline
\end{tabular}

Table 55: Design parameters of the high field interaction region magnets. 


\begin{tabular}{|lrrl|}
\hline RMS ERROR & ARC & IR & \\
QUADRUPOLES & & & \\
Transverse offset & 0.25 & 0.2 & $\mathrm{~mm}$ \\
BPM to quad offset & 0.15 & 0.1 & $\mathrm{~mm}$ \\
Roll & 0.5 & 0.5 & $\mathrm{mrad}$ \\
Field Error $\Delta K / K$ & 0.5 & 0.1 & $10^{-3}$ \\
& & & \\
DIPOLES & & & \\
Roll & 0.5 & 0.5 & $\mathrm{mrad}$ \\
Field Error $\Delta B / B$ & 0.5 & 0.5 & $10^{-3}$ \\
& & & \\
\hline
\end{tabular}

Table 56: Tolerance summary for magnet alignment

\begin{tabular}{|lrl|}
\hline & & \\
ARC CORRECTORS & & \\
Dipole magnetic length & 1.05 & $\mathrm{~m}$ \\
Dipole max strength & 2.3 & $\mathrm{Tm}$ \\
Skew quad length & 0.95 & $\mathrm{~m}$ \\
Skew quad max strength & 95 & $\mathrm{~T}$ \\
Sextupole magnetic length & 0.95 & $\mathrm{~m}$ \\
Sextupole max strength & 3700 & $\mathrm{~T} / \mathrm{m}$ \\
& & \\
IR CORRECTORS & & \\
Magnetic length & 2.5 & $\mathrm{~m}$ \\
Dipole max strength & 8.6 & $\mathrm{Tm}$ \\
Skew quad max strength & 95 & $\mathrm{~T}$ \\
& & \\
\hline
\end{tabular}

Table 57: Corrector magnetic lengths and strengths 


\subsection{Magnet Reference Harmonics}

The expansion for the field error in a dipole magnet is written in terms of coefficients $\left(b_{n}, a_{n}\right)$ as

$$
\Delta\left(B_{y}+i B_{x}\right)=B_{0} 10^{-4} \sum_{n=1}\left(b_{n}+i a_{n}\right)\left(\frac{x+i y}{r_{0}}\right)^{n}
$$

where $B_{0}$ is the main dipole field and $r_{0}=10 \mathrm{~mm}$ is the reference radius in all cases. Similarly, for a quadrupole

$$
\Delta\left(B_{y}+i B_{x}\right)=G_{0} r_{0} 10^{-4} \sum_{n=2}\left(b_{n}+i a_{n}\right)\left(\frac{x+i y}{r_{0}}\right)^{n}
$$

where $G_{0}$ is the main quadrupole field gradient. In the following tables, for each set of magnets, $b_{n}\left(a_{n}\right)$ is the mean or systematic value of the normal (skew) harmonic, $\Delta\left(b_{n}\right)$ is the uncertainty of the systematic normal harmonic, and $\sigma\left(b_{n}\right)$ is the standard deviation of the normal harmonic. The convention in which $n=2$ is sextupole is used throughout.

\begin{tabular}{|rrrrrrr|}
\hline $\mathrm{n}$ & $b_{n}$ & $\Delta b_{n}$ & $\sigma\left(b_{n}\right)$ & $a_{n}$ & $\Delta a_{n}$ & $\sigma\left(a_{n}\right)$ \\
\hline & & .5 & 1.2 & .005 & .5 & 1.2 \\
1 & & & & & & \\
2 & -2.0 & 1.0 & .85 & & .3 & .85 \\
3 & & .2 & .3 & -.001 & .2 & .3 \\
4 & -.5 & .2 & .12 & & .2 & .12 \\
5 & & & .05 & -.002 & & .05 \\
6 & -.001 & & .02 & & & .02 \\
7 & & & .008 & .011 & & .008 \\
8 & -.046 & & .005 & & & .005 \\
9 & & & .001 & .003 & & .001 \\
& & & & & & \\
\hline
\end{tabular}

\begin{tabular}{|rrrrrrr|}
\hline $\mathrm{n}$ & $b_{n}$ & $\Delta b_{n}$ & $\sigma\left(b_{n}\right)$ & $a_{n}$ & $\Delta a_{n}$ & $\sigma\left(a_{n}\right)$ \\
\hline & & .5 & 1.2 & & .5 & 1.2 \\
1 & & .5 & .6 & & .3 & .6 \\
2 & & .2 & .3 & -.001 & .2 & .3 \\
3 & & .1 & & .05 & .1 \\
4 & -.001 & .05 & .05 & -.002 & & .05 \\
5 & & & .02 & & & .02 \\
6 & -.001 & & .008 & .011 & & .008 \\
7 & & & .005 & & & .005 \\
8 & -.046 & & .001 & .003 & & .001 \\
9 & & & & & & \\
\hline
\end{tabular}

Table 58: Arc dipole body harmonics, v1.0. At injection with $B=2 \mathrm{~T}$ (top), and at storage with $B=10 \mathrm{~T}$ (bottom).

The injection energy dropped from the $20 \mathrm{TeV}$ value used for v1.0 harmonics to $10 \mathrm{TeV}$. The injection field reduces from $2.28 \mathrm{~T}$ to $1.14 \mathrm{~T}$ and injection field gradient from $90 \mathrm{~T} / \mathrm{m}$ to $45 \mathrm{~T} / \mathrm{m}$. The components of field harmonics related to the coil magnetization effect at injection have been checked and corrected if necessary. 


\begin{tabular}{|c|c|c|c|c|c|c|}
\hline $\mathrm{n}$ & $b_{n}$ & $\Delta b_{n}$ & $\sigma\left(b_{n}\right)$ & $a_{n}$ & $\Delta a_{n}$ & $\sigma\left(a_{n}\right)$ \\
\hline 2 & & .75 & 1.875 & & .75 & 1.875 \\
\hline 3 & .004 & .5 & .875 & & .5 & .875 \\
\hline 4 & & .125 & .375 & & .125 & .375 \\
\hline 5 & -2.5 & .25 & .25 & & .125 & .25 \\
\hline 6 & & & .075 & & & .075 \\
\hline 7 & -.0035 & & .025 & & & .025 \\
\hline 8 & & & .025 & & & .025 \\
\hline 9 & .1 & .025 & .0125 & & & .0125 \\
\hline $\mathrm{n}$ & $b_{n}$ & $\Delta b_{n}$ & $\sigma\left(b_{n}\right)$ & $a_{n}$ & $\Delta a_{n}$ & $\sigma\left(a_{n}\right)$ \\
\hline 2 & & .75 & 1.875 & .175 & .75 & 1.875 \\
\hline 3 & .03 & .50 & .875 & & .5 & .875 \\
\hline 4 & & .125 & .375 & & .125 & .375 \\
\hline 5 & .03 & .075 & .2 & & .075 & .2 \\
\hline 6 & & & .075 & & & .075 \\
\hline 7 & -.0035 & & .025 & & & .025 \\
\hline 8 & & & .025 & & & .025 \\
\hline 9 & -.004 & .025 & .0125 & & & .0125 \\
\hline
\end{tabular}

Table 59: Arc quadrupole body harmonics, v1.0. At injection with $G=80 \mathrm{~T} / \mathrm{m}$ (top), and at storage with $G=400 \mathrm{~T} / \mathrm{m}$ (bottom). 


\begin{tabular}{|rrrrrrr|}
\hline $\mathrm{n}$ & $b_{n}$ & $\Delta b_{n}$ & $\sigma\left(b_{n}\right)$ & $a_{n}$ & $\Delta a_{n}$ & $\sigma\left(a_{n}\right)$ \\
\hline & & & & & .5 & 1.2 \\
1 & & .5 & 1.2 & & .3 & .85 \\
2 & -2.0 & 1.0 & .85 & & .05 & .35 \\
3 & & .05 & .35 & & .2 & .12 \\
4 & .5 & .2 & .12 & & .002 & .05 \\
5 & -.006 & .006 & .05 & & & .02 \\
6 & -.004 & .007 & .02 & & .005 & .008 \\
7 & .015 & .015 & .008 & .01 & .005 \\
8 & -.065 & .065 & .005 & & & .005 \\
9 & -.006 & .006 & .001 & .002 & .001 & .001 \\
& & & & & & \\
\hline
\end{tabular}

\begin{tabular}{|rrrrrrr|}
\hline $\mathrm{n}$ & $b_{n}$ & $\Delta b_{n}$ & $\sigma\left(b_{n}\right)$ & $a_{n}$ & $\Delta a_{n}$ & $\sigma\left(a_{n}\right)$ \\
\hline & & & & & .5 & 1.2 \\
1 & & .5 & 1.2 & & .3 & .6 \\
2 & & .5 & .6 & & .05 & .3 \\
3 & & .05 & .3 & & .05 & .1 \\
4 & & .05 & .1 & & .002 & .05 \\
5 & -.006 & .006 & .05 & & & .02 \\
6 & -.004 & .007 & .02 & & .005 & .008 \\
7 & .015 & .015 & .008 & .01 & .005 \\
8 & -.065 & .065 & .005 & & & .005 \\
9 & -.006 & .006 & .001 & .002 & .001 & .001 \\
& & & & & & \\
\hline
\end{tabular}

Table 60: Arc dipole body harmonics, v1.1. At injection with $B=1.14 \mathrm{~T}$ (top), and at storage with $B=10 \mathrm{~T}$ (bottom). 


\begin{tabular}{|lllllll|}
\hline $\mathrm{n}$ & $b_{n}$ & $\Delta b_{n}$ & $\sigma\left(b_{n}\right)$ & $a_{n}$ & $\Delta a_{n}$ & $\sigma\left(a_{n}\right)$ \\
\hline & & & & & & \\
2 & & .25 & 1.875 & .25 & 1.875 \\
3 & & .25 & .875 & .25 & .875 \\
4 & & .125 & .375 & .125 & .375 \\
5 & -1.25 & .25 & .25 & .125 & .25 \\
6 & & & .075 & & .075 \\
7 & -.004 & & .025 & & .025 \\
8 & & & .025 & & .025 \\
9 & .075 & .025 & .013 & .013 & .0125 \\
& & & & & \\
\hline
\end{tabular}

\begin{tabular}{|lllllll|}
\hline $\mathrm{n}$ & $b_{n}$ & $\Delta b_{n}$ & $\sigma\left(b_{n}\right)$ & $a_{n}$ & $\Delta a_{n}$ & $\sigma\left(a_{n}\right)$ \\
\hline & & & & & & \\
2 & & .5 & 1.875 & .175 & .5 & 1.875 \\
3 & & .25 & .875 & & .25 & .875 \\
4 & & .125 & .375 & & .125 & .375 \\
5 & .025 & .025 & .2 & & .025 & .2 \\
6 & & & .075 & & & .075 \\
7 & -.0035 & & .025 & & .025 \\
8 & & & .025 & & .025 \\
9 & -.004 & .003 & .0125 & & .0125 \\
& & & & & & \\
\hline
\end{tabular}

Table 61: Arc quadrupole body harmonics, v1.1. At injection with $G=45 \mathrm{~T} / \mathrm{m}$ (top), and at storage with $G=400 \mathrm{~T} / \mathrm{m}$ (bottom).

\begin{tabular}{|c|c|c|c|c|c|c|}
\hline $\mathrm{n}$ & $b_{n}$ & $\Delta b_{n}$ & $\sigma\left(b_{n}\right)$ & $a_{n}$ & $\Delta a_{n}$ & $\sigma\left(a_{n}\right)$ \\
\hline 2 & & 2 & 2 & 3 & 3 & 1 \\
\hline 3 & 2 & 2 & 1 & & 1 & 1 \\
\hline 4 & & 1 & 1 & 1.5 & 1.5 & 1 \\
\hline 5 & 1 & 1 & .5 & & 1 & .2 \\
\hline 6 & & .1 & .1 & .5 & .5 & .5 \\
\hline 7 & .5 & .5 & .1 & & .2 & .1 \\
\hline 8 & & .1 & .1 & .3 & .3 & .1 \\
\hline 9 & .5 & .5 & .1 & & .1 & .1 \\
\hline
\end{tabular}

Table 62: Interaction region quadrupole integrated harmonics for Q1 and Q2, v1.0. 


\section{References}

[1] P. Limon et al, Design Study for a Staged VLHC, Fermilab-TM-2149, May 2001.

[2] J. Johnstone et al, Lattice Design of the VLHC Rings, PAC 01, Chicago, 2001.

[3] S. Peggs et al, Lattices for a high-field 30 TeV hadron collider, Snowmass, 1996.

[4] S. Peggs et al, Lattice optimization for a Really Large Hadron Collider, EPAC 96, Barcelona, 1996.

[5] S. Peggs, F. Dell, Tolerable systematic errors in Really Large Hadron Colliders, Snowmass, 1996.

[6] D. Neuffer, Particle Accelerators 23 (1988), 21.

[7] J.P. Koutchouk, The LHC Dynamic Aperture, PAC 99, New York, 1999.

[8] F. Ostiguy, personal communication, March 19, 2001.

[9] P. Schlabach, Cancellation of the harmonics of the two cold masses in a Q2, Fermilab TD Note, 2000.

[10] G. Ambrosio, personal communication, March 22, 2001

[11] T. Sen, Alignment tolerances of IR quadrupoles in the LHC, FNAL-Conf-99-304, 1999.

[12] Y. Nosochkov, D. Ritson, Provision of IP crossing angles for the SSC, SSCL-Preprint-367, 1993.

[13] T. Sen et al, Chromaticity Correction for the SSC Collider Rings, PAC 93, p. 143, 1993.

[14] V. Shiltsev, Proc. ICFA Workshop on Ground Motion, SLAC, 2000; http://www-project.slac.stanford.edu/ls/wkshp/GM2000

[15] B.Baklakov et al, VLHC/NLC Slow Ground Motion Studies in Illinois, PAC 01, Chicago, 2001.

[16] B. Baklakov et al, PRST Accel. Beams, 031001, 1998.

[17] V. Shiltsev, FNAL TM-1987, 1996.

[18] A. Burov et al, Beam Stability Issues in a Very Large Hadron Collider, p. 194 NIMA 450, 2000.

[19] See for example, K.Y. Ng, Impedances and Collective instabilities of the Tevatron at Run II, Fermilab Report TM-2055, 1998.

[20] M. Blaskiewicz, paper in Stability issues for the high field VLHC, VLHC Instability Workshop, SLAC, 2001; http://www.slac.stanford.edu/ achao/VLHCworkshop.html.

[21] A. Burov et al., Nucl. Instrum. Meth. A450, 194, 2000.

[22] W. Foster, private communication, Staged VLHC Parameter List Version 0.4 2/23/01, 2001.

[23] S. Krinsky and J.M. Wang, Particle Accelerators 17, 109, 1985.

[24] K.Y. Ng, Stability Issues of the VLHC Rings, presented at the VLHC Instability Workshop, SLAC Report SLAC-Pub-8800, SLAC, 2001; http://www.slac.stanford.edu/ achao/VLHCworkshop.html.

[25] A. Hofmann and B. Zotter, Combined-function electron storage ring, CERN/ISR-GS/76-28, 1976.

[26] D.P. Barber and A. De Roerk (editors), DESY-PROC-1999-03, 1999.

[27] V.A. Anferov, Phys.Rev.Lett. 83 14, p.2738-2740, 1999.

[28] S.R. Mane, Phys.Rev. A 36 (1) p.105-130, 1986.

[29] M. Vogt, PhD-thesis, University of Hamburg, FRG, 2000; also: DESY-THESIS-2000-054, 2000; and http://wwwmpy.desy.de/proton pol/hoffpaper/00vogt.ps.gz 
[30] Ya.S. Derbenev and A.M. Kondratenko, Sov.Phy. JETP 35 (2) pp.230, (1972) and Sov.Phy. JETP 37 (6) pp.968, 1973.

[31] D.P. Barber et al, DESY-M-92-04 (1992), 2-nd revised version, 1999.

[32] K. Yokoya, DESY-86-57, 1986.

[33] G.H. Hoffstaetter, Habilitation-thesis, Technical University Darmstadt, FRG, 2000; (to be published by Springer Verlag).

[34] D.P. Barber et al, DESY-HERA-96-07, 1996.

[35] G.H. Hoffstaetter and M. Vogt, The SPRINT Manual, to be published in 2001.

[36] J.A. Johnstone, private communication, 2001.

[37] S.Y. Lee, Spin Dynamics and Snakes in Synchrotrons, World Scientific, 1997.

[38] K. Yokoya, DESY-99-006, 1999.

[39] E. Keil, R. Talman, Scaling of Luminosity Data Between $e^{+} e-$ Storage Rings, Particle Accelerators Vol. 14 pp. $109-118$, 1983.

[40] J. Wei, Intra-beam scattering scaling for Very Large Hadron Colliders, BNL C-A/AP/38, 2001.

[41] J.Wei, Intra-Beam Scattering Scaling for VLHCs, PAC 01, Chicago, 2001.

[42] S. Peck, Minimizing Chromaticity in Interaction Regions with Long Weak Quadrupoles Snowmass 1984, p. 384.

[43] J. Wei, S. Peggs, Interaction region analysis for a high-field hadron collider, Snowmass, 1996.

[44] S. Peggs et al, Flat beams in a 50 TeV hadron collider, Vancouver PAC 97, 1997.

[45] Field Quality of the Fermilab Nb3Sn High Field Dipole Model, PAC 01, Chicago, 2001.

[46] W. Fischer, An Experimental Study on the Long-term Stability of Particle Motion in Hadron Storage Rings, PhD thesis Hamburg University, DESY 95-235 (1995) and CERN SL/96-10 (AP), 1996.

[47] W. Fischer et al, Dynamic Aperture Experiment at a Synchrotron, Phys. Rev. E, Vol. 55, Number 3, p. $3507,1997$.

[48] Condon \& Odishaw, Handbook of Physics, pg 4-72, 1967.

[49] G. McIntyre, private communication, 2001.

[50] J.M. Wang, SLAC summer school, 1985.

[51] J. Marriner, FNAL tech note, September, 1997.

[52] M. Blaskiewicz, W.T. Weng, Phys. Rev. E, Vol 50, \# 5, p 4030, 1994.

[53] Y.H. Chin, CERN/LEP-TH/88-05, 1988.

[54] Synchrotron Radiation Issues in the VLHC, PAC 01, Chicago, 2001.

[55] L. Vos, LHC Project Note 150, CERN, 1998.

[56] S.Y. Zhang, BNL C-AD AP Tech. Note, in preparation, 2001.

[57] H. Seiler, J. Appl. Phys. 54, R1, 1983.

[58] O. Gröbner, LHC Project Report 127, 1997.

[59] T. Sjoestrand, PYTHIA 5.7 and JETSET 7.4, Physics and Manual, Computer Physics Commun. 82 (1994) 74.

[60] A. Chao (Editor), Stability issues for the high field VLHC, VLHC Instability Workshop, SLAC, 2001; http://www.slac.stanford.edu/ achao/VLHCworkshop.html. 